University of New Hampshire

University of New Hampshire Scholars' Repository

Fall 2008

\title{
A comparative analysis of control techniques for formation flying spacecraft in an Earth/Moon-Sun L(2)-centered lissajous orbit
}

Michael Farrar

University of New Hampshire, Durham

Follow this and additional works at: https://scholars.unh.edu/thesis

\section{Recommended Citation}

Farrar, Michael, "A comparative analysis of control techniques for formation flying spacecraft in an Earth/ Moon-Sun L(2)-centered lissajous orbit" (2008). Master's Theses and Capstones. 380.

https://scholars.unh.edu/thesis/380

This Thesis is brought to you for free and open access by the Student Scholarship at University of New Hampshire Scholars' Repository. It has been accepted for inclusion in Master's Theses and Capstones by an authorized administrator of University of New Hampshire Scholars' Repository. For more information, please contact Scholarly.Communication@unh.edu. 


\title{
A COMPARATIVE ANALYSIS OF CONTROL TECHNIQUES FOR FORMATION FLYING SPACECRAFT IN AN EARTH/MOON-SUN $L_{2}$-CENTERED LISSAJOUS ORBIT
}

\author{
BY \\ MICHAEL FARRAR \\ B.S., University of New Hampshire, 2006
}

THESIS

Submitted to the University of New Hampshire

in Partial Fulfillment of

the Requirements for the Degree of

Master of Science

in

Mechanical Engineering

September 2008 
UMI Number: 1459493

\section{INFORMATION TO USERS}

The quality of this reproduction is dependent upon the quality of the copy submitted. Broken or indistinct print, colored or poor quality illustrations and photographs, print bleed-through, substandard margins, and improper alignment can adversely affect reproduction.

In the unlikely event that the author did not send a complete manuscript and there are missing pages, these will be noted. Also, if unauthorized copyright material had to be removed, a note will indicate the deletion.

(®)

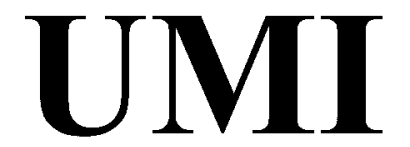

UMI Microform 1459493

Copyright 2008 by ProQuest LLC.

All rights reserved. This microform edition is protected against unauthorized copying under Title 17, United States Code.

ProQuest LLC

789 E. Eisenhower Parkway

PO Box 1346

Ann Arbor, Ml 48106-1346 
This thesis has been examined and approved.

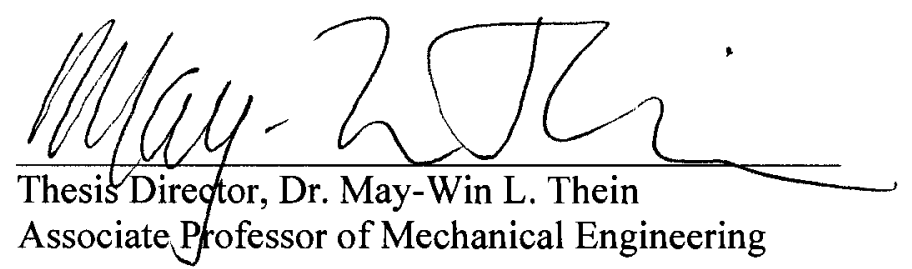

Dr. Barry Fussell

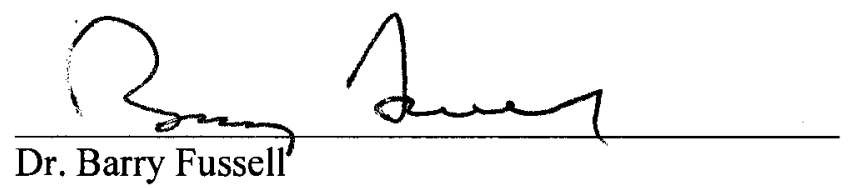

Professor of Mechanical Engineering

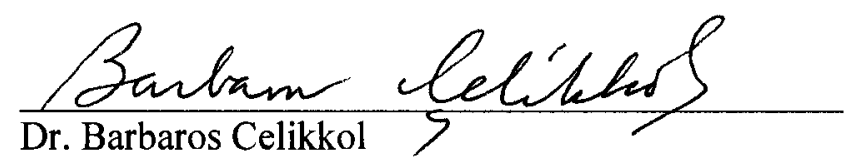

Professor of Mechanical Engineering

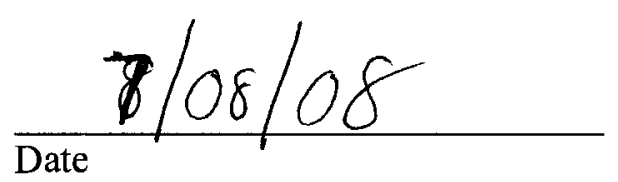




\section{ACKNOWLEDGMENTS}

I would like to thank everyone who has supported me over the last two years while I worked on this research. My advisor, Prof. May-Win Thein, for taking me on as her graduate student and putting up with my antics. Dave Folta at NASA Goddard Space Flight Center for offering me the opportunity to assist in this research.

And special thanks to my family and friends. 


\section{TABLE OF CONTENTS}

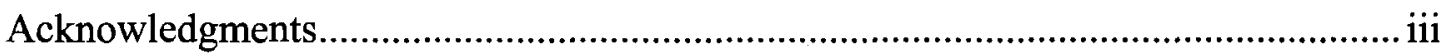

List of Figures ................................................................................................ xi

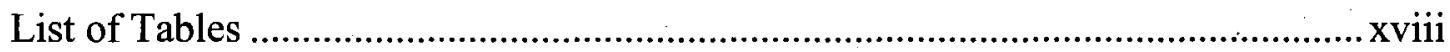

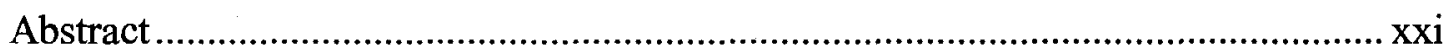

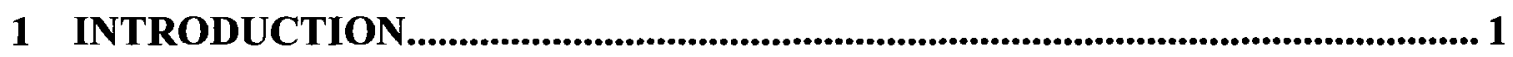

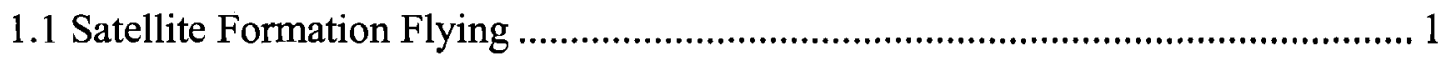

1.2 Examples of Past, Current and Future Missions ............................................... 3

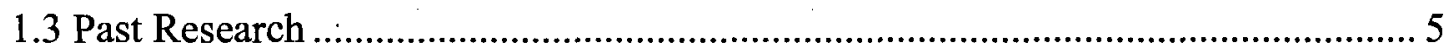

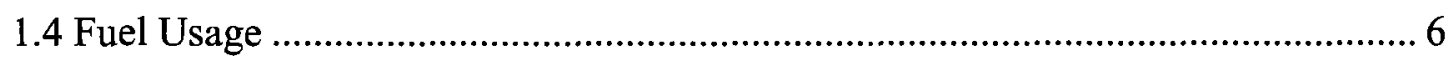

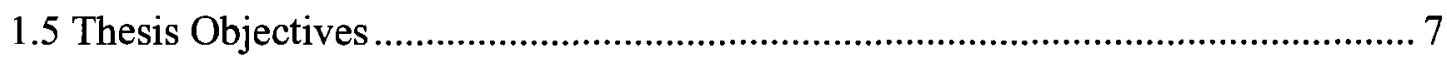

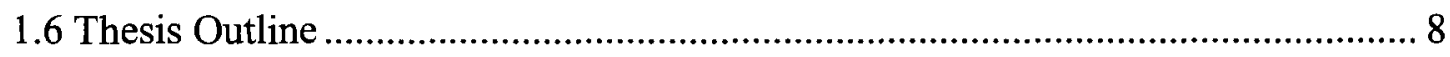

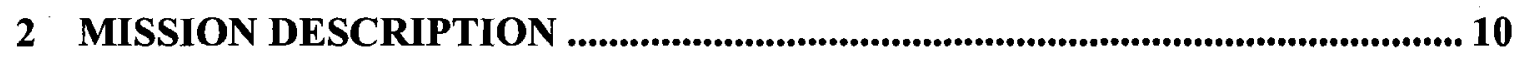

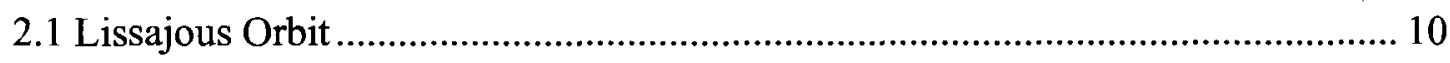

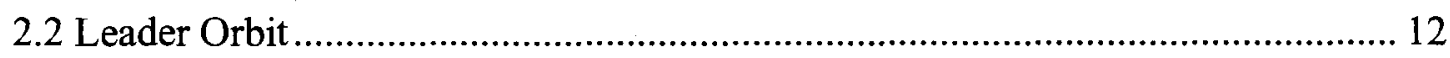

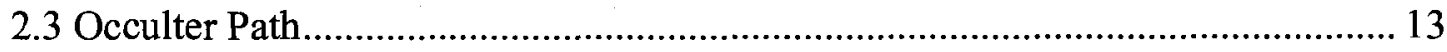

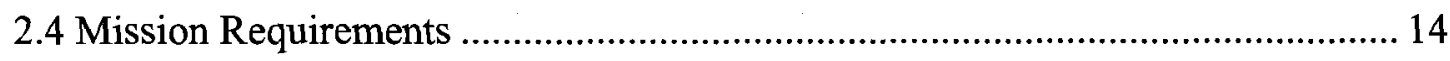

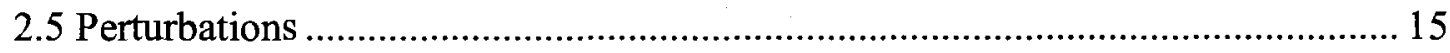

3 THE CIRCULAR RESTRICTED THREE BODY PROBLEM (CR3BP) ......... 17

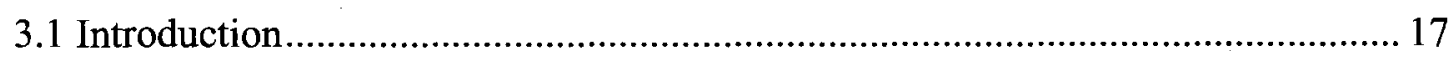

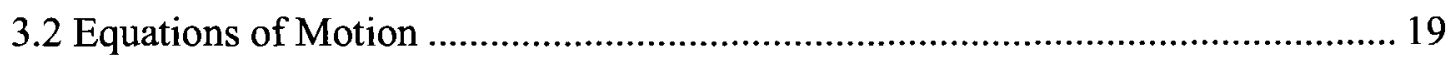




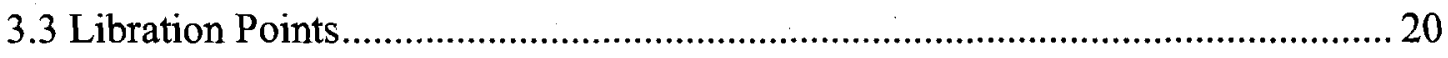

3.4 Linearized Equations of Motion ……………………...................................... 22

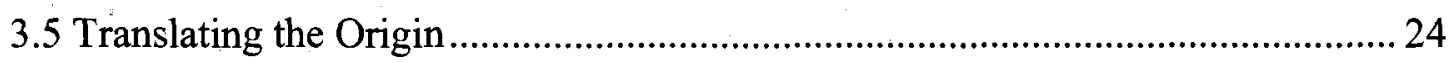

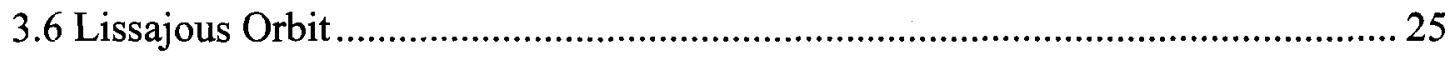

4 DISTURBANCES AND PERTURBATIONS ............................................................ 29

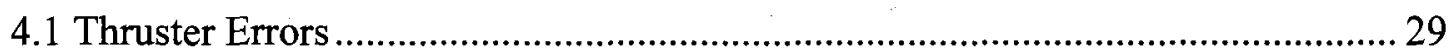

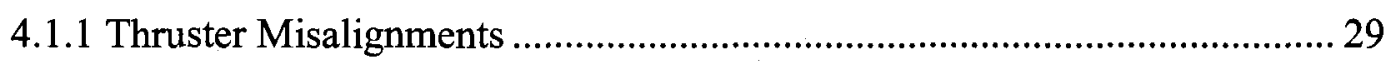

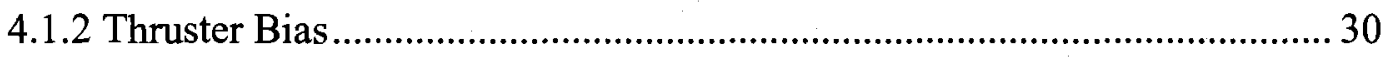

4.2 Gravitational Perturbations ............................................................................... 30

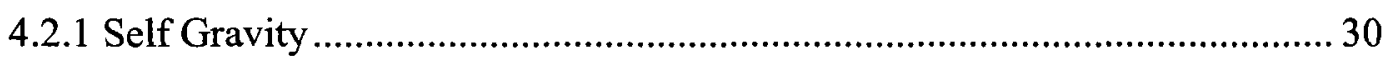

4.2.2 Gravitational Effects of Jupiter ..................................................................... 31

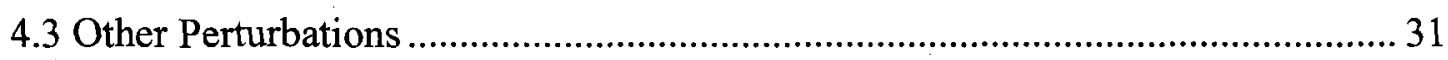

4.3.1 Solar Radiation Pressure ................................................................................. 31

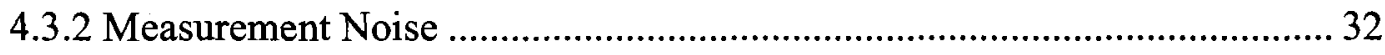

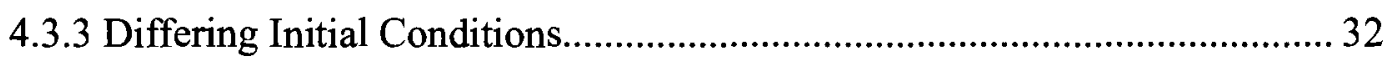

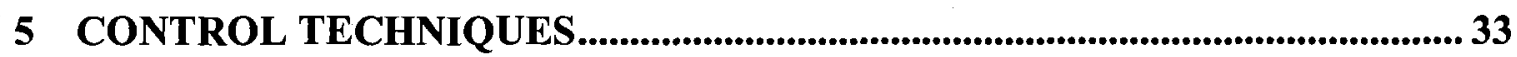

5.1 Investigated Control Techniques ....................................................................... 33

5.1.1 Properties of PID Control ............................................................................ 34

5.1.2 Properties of Linear Quadratic Regulator ..................................................... 35

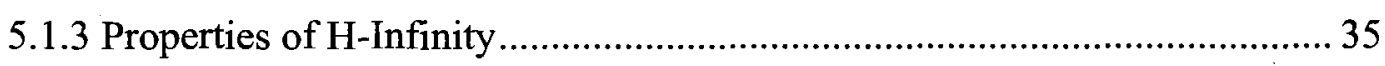

5.1.4 Properties of Sliding Mode Control ............................................................. 36

5.2 Other Control Techniques................................................................................. 36 


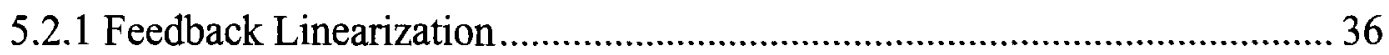

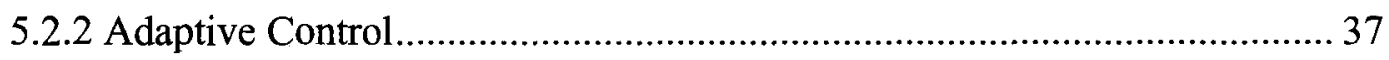

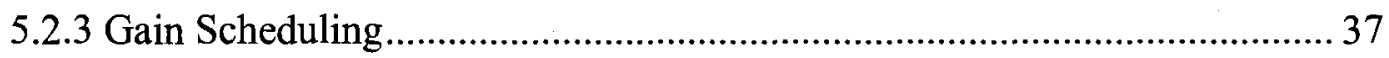

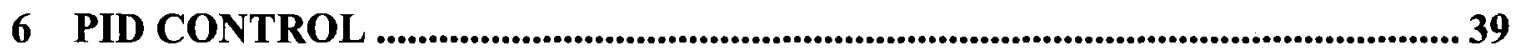

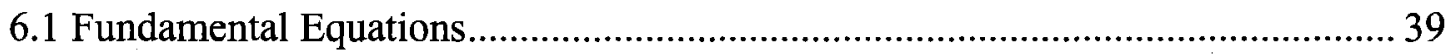

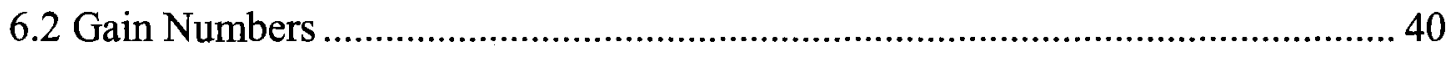

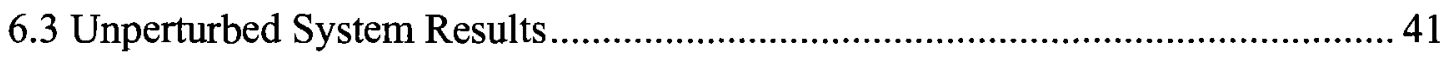

6.4 Thruster Misalignment Results ......................................................................... 44

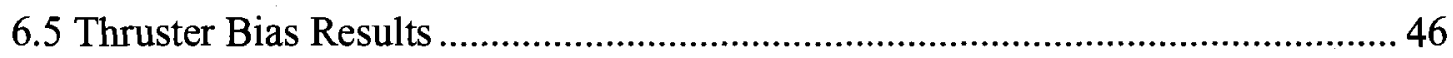

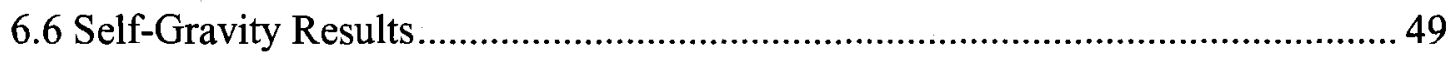

6.7 Jupiter Gravity Results................................................................................ 51

6.8 Solar Radiation Pressure Results ...................................................................... 53

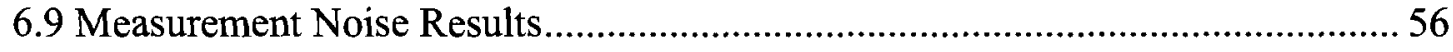

6.10 Differing Initial Conditions Results............................................................... 58

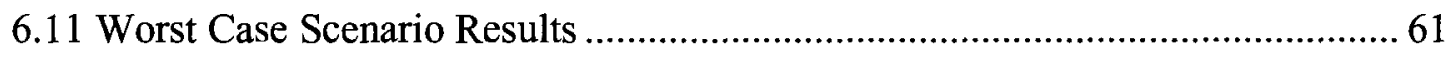

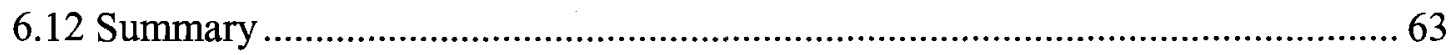

7 LINEAR QUADRATIC REGULATOR............................................................... 65

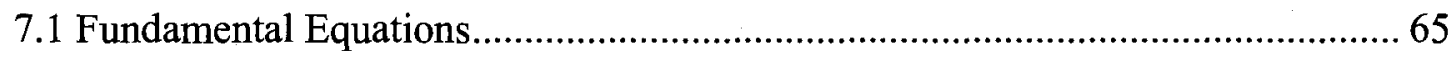

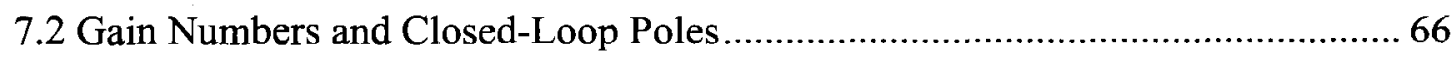

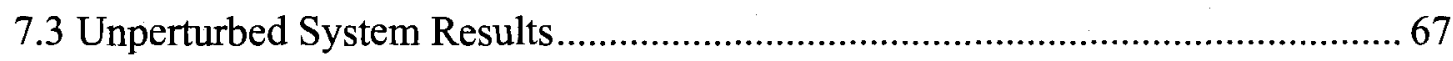

7.4 Thruster Misalignment Results ............................................................................. 70

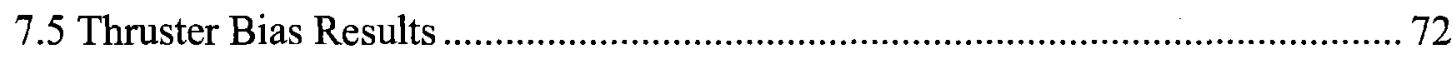




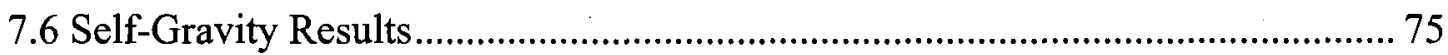

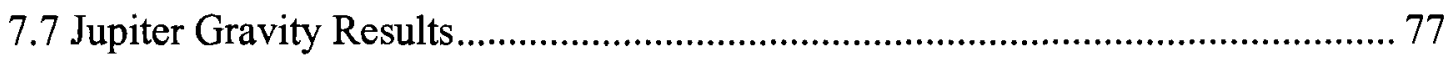

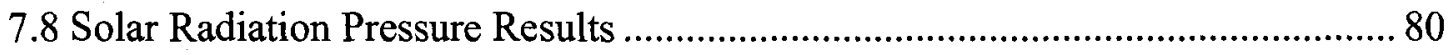

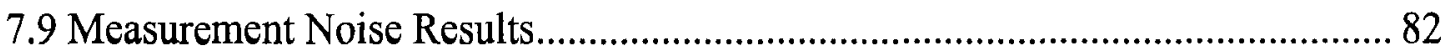

7.10 Differing Initial Conditions Results................................................................... 85

7.11 Worst Case Scenario Results ............................................................................... 87

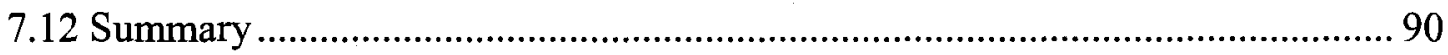

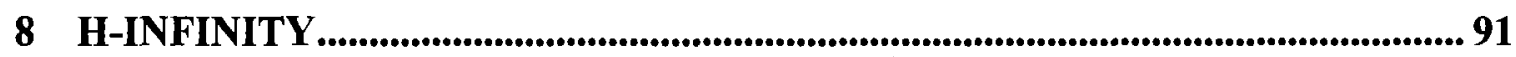

8.1 Fundamental Equations.................................................................................... 91

8.1.1 Cost Function ..................................................................................... 91

8.1.2 Minimization Problem ............................................................................. 92

8.1.3 Maximization Problem................................................................................. 92

8.1.4 Two-Point-Boundary Problem...................................................................... 92

8.1.5 Control Law with Disturbance Contribution ............................................... 94

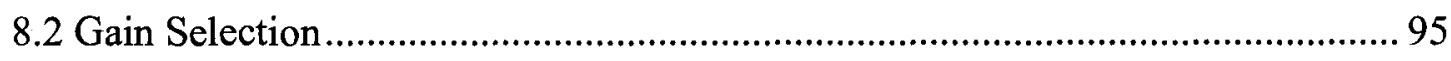

8.3 Unperturbed System Results............................................................................. 96

8.4 Thruster Misalignment Results ........................................................................ 99

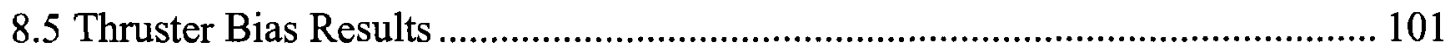

8.6 Self-Gravity Results...................................................................................... 104

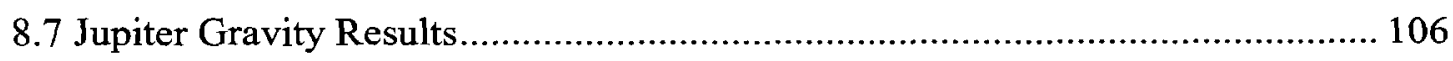

8.8 Solar Radiation Pressure Results ....................................................................... 109

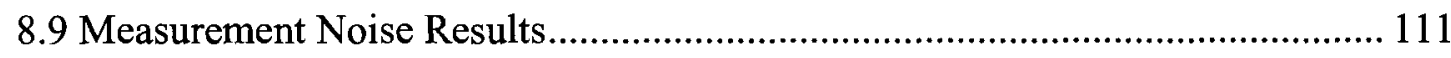

8.10 Differing Initial Conditions Results .................................................................. 114 


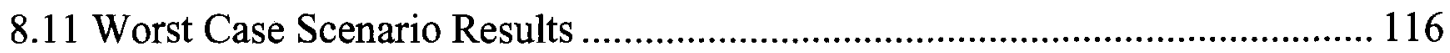

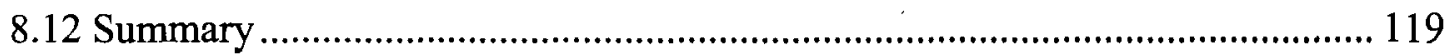

9 SLIDING MODE CONTROL ......................................................................... 120

9.1 Fundamental Equations.................................................................................. 120

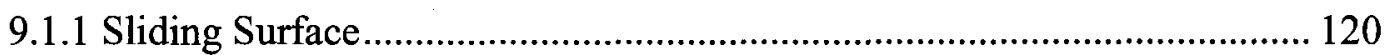

9.1.2 Sliding Surface Hyperplane Matrix ……………………………............. 122

9.1.3 Optimal Sliding Mode Control ............................................................... 123

9.1.4 Chattering Effect........................................................................................ 124

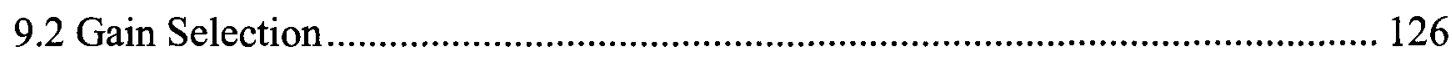

9.3 Unperturbed System Results........................................................................... 127

9.4 Thruster Misalignment Results ........................................................................ 130

9.5 Thruster Bias Results .................................................................................... 132

9.6 Self-Gravity Results...................................................................................... 135

9.7 Jupiter Gravity Results.................................................................................. 137

9.8 Solar Radiation Pressure Results ....................................................................... 140

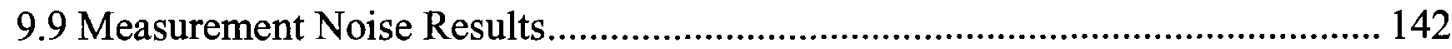

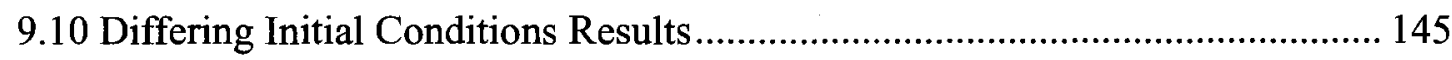

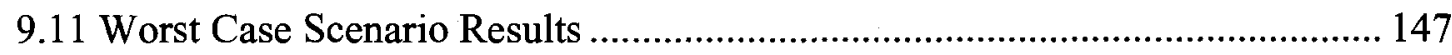

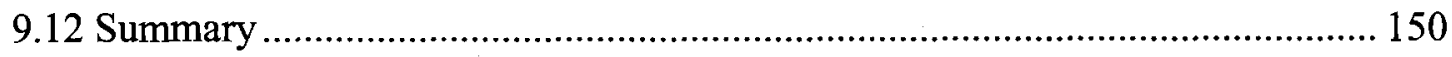

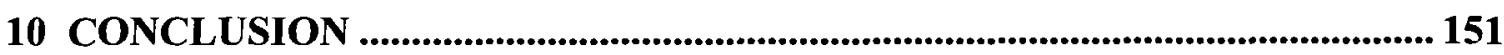

10.1 Comparison of Controllers............................................................................ 151

10.1.1 Unperturbed Results........................................................................ 151 
10.1.2 Thruster Errors and External Perturbations .............................................. 151

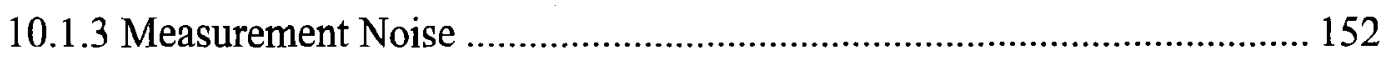

10.1.4 Differing Initial Conditions Results....................................................... 153

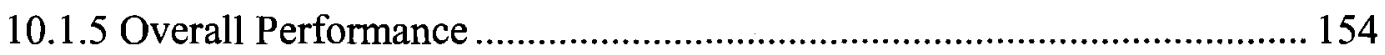

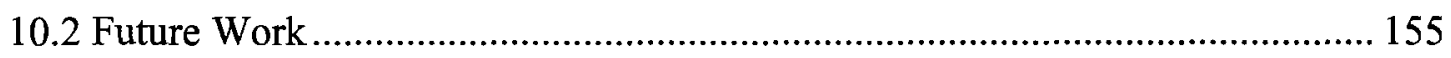

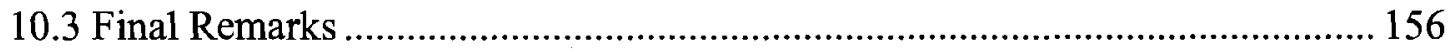

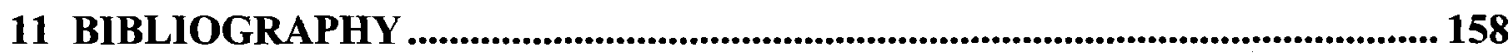

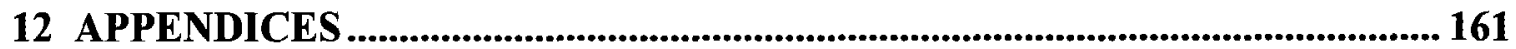

A CR3BP AND DISTURBANCE MODELS......................................................... 162

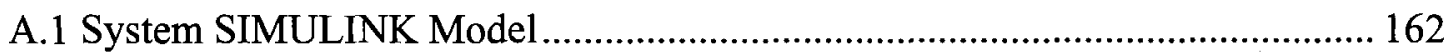

A.1.1 Embedded MATLAB Functions ............................................................. 165

A.2 Reference SIMULINK Model ................................................................... 166

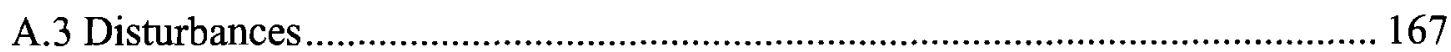

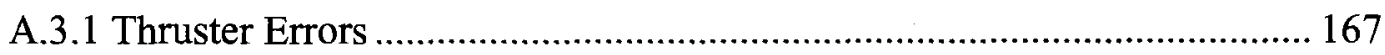

A.3.2 External Perturbations............................................................................ 168

A.4 Common m-File Code................................................................................ 170

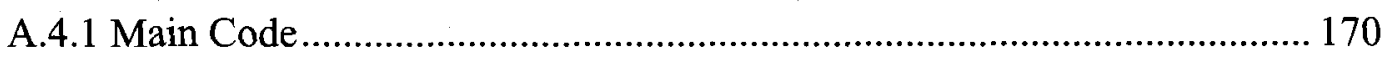

A.4.2 Reference Orbit Code......................................................................... 170

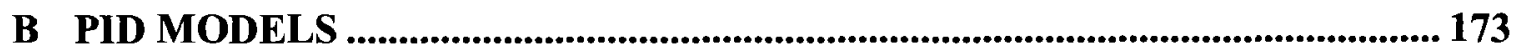

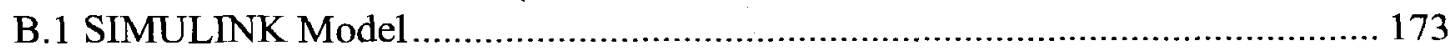

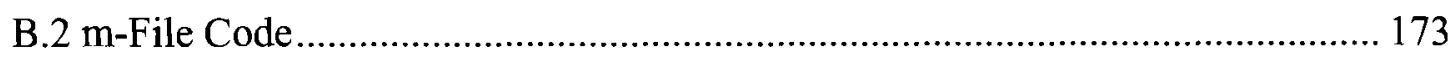

C LQR MODELS.......................................................................................................... 175

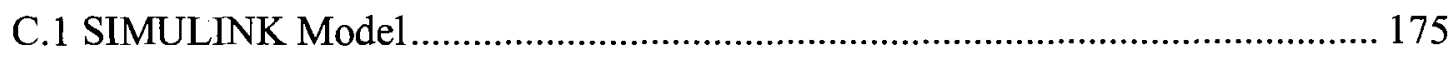




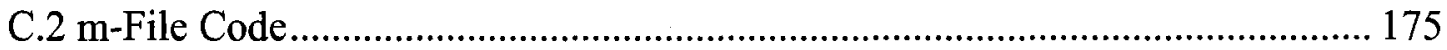

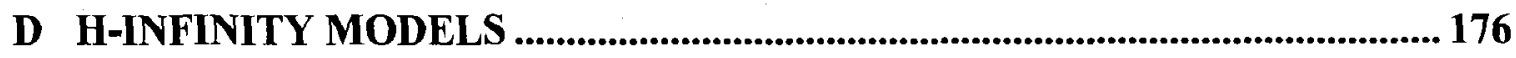

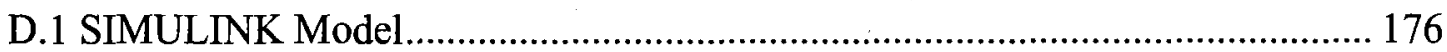

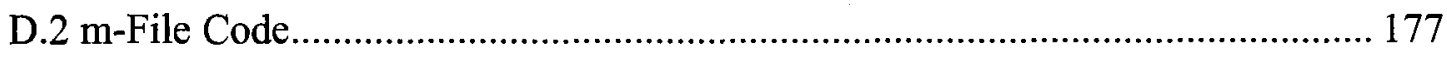

E SMC MODELS ……................................................................................................ 179

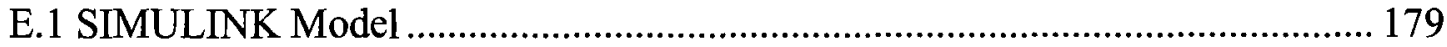

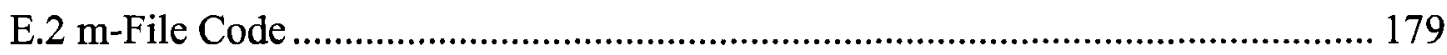

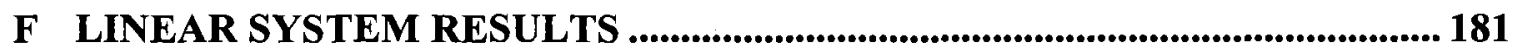




\section{LIST OF FIGURES}

Figure 1.1 - Location of libration points 3

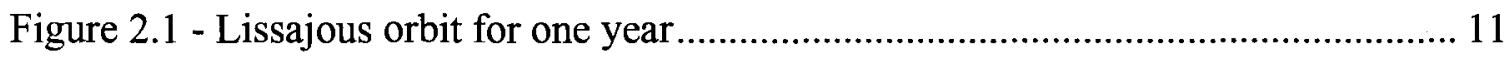

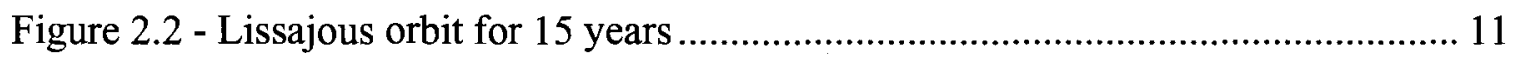

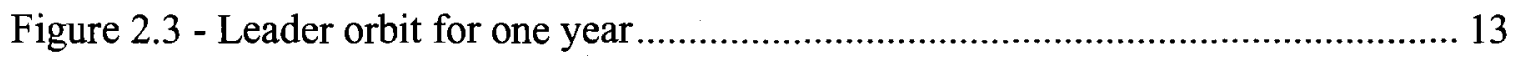

Figure 2.4 - Leader and occulter orbits with respect to Earth.................................... 14

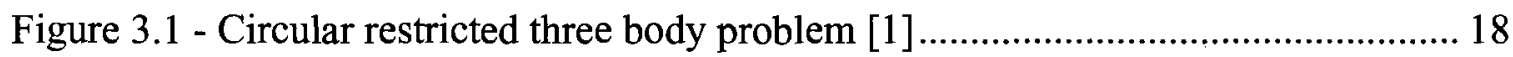

Figure 3.2 - Location of Earth/Moon-Sun libration points (not to scale), where $M_{1}$ represents the Sun and $M_{2}$ represents the Earth/Moon system [1] .............................. 21

Figure 3.3 - Reference frame with translated origin ........................................... 24

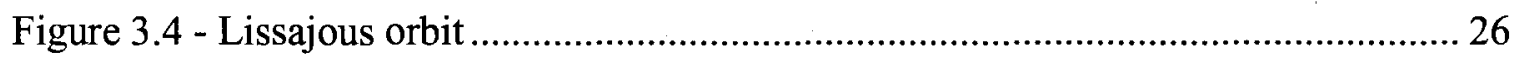

Figure 3.5 - Lissajous orbit with respect to the Earth/Moon system ............................. 27

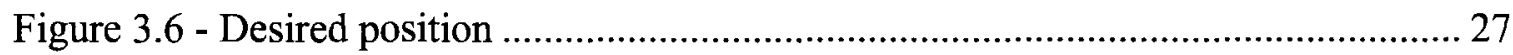

Figure 5.1 - Formation flying closed-loop control system ...................................... 33

Figure 6.1 - Simplified PID block diagram ...................................................... 40

Figure 6.2 - PID position errors for 1 year (unperturbed)..................................... 41

Figure 6.3 - PID velocity errors for 1 year (unperturbed) ........................................ 42

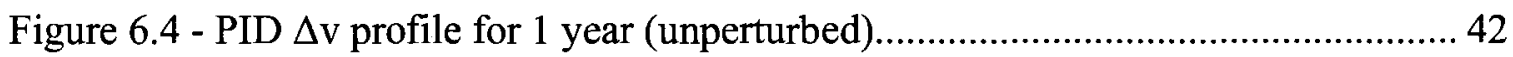

Figure 6.5 - PID position errors for 1 year (thruster misalignments) .......................... 44

Figure 6.6 - PID velocity errors for 1 year (thruster misalignments) ........................ 45 
Figure 6.7 - PID $\Delta v$ profile for 1 year (thruster misalignments)

Figure 6.8 - PID position errors for 1 year (thruster bias) ............................................. 47

Figure 6.9 - PID velocity errors for 1 year (thruster bias) .............................................. 47

Figure 6.10 - PID $\Delta \mathrm{v}$ profile for 1 year (thruster bias) …………...................................... 48

Figure 6.11 - PID position errors for 1 year (self-gravity) ………....................................49

Figure 6.12 - PID velocity errors for 1 year (self-gravity) ............................................. 50

Figure 6.13 - PID $\Delta \mathrm{v}$ profile for 1 year (self-gravity) ………………............................ 50

Figure 6.14 - PID position errors for 1 year (Jupiter) ……………………………...... 51

Figure 6.15 - PID velocity errors for 1 year (Jupiter) ……………............................... 52

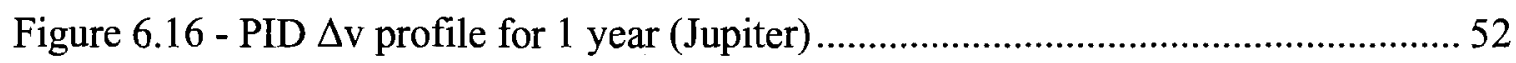

Figure 6.17 - PID position errors for 1 year (solar radiation pressure).............................. 54

Figure 6.18 - PID velocity errors for 1 year (solar radiation pressure).............................. 54

Figure 6.19 - PID $\Delta \mathrm{v}$ profile for 1 year (solar radiation pressure).................................... 55

Figure 6.20 - PID position errors for 1 year (measurement noise) .................................... 56

Figure 6.21 - PID velocity errors for 1 year (measurement noise) ................................... 57

Figure 6.22 - PID $\Delta v$ profile for 1 year (measurement noise) ………………................. 57

Figure 6.23 - PID position errors for 1 year (differing initial conditions)......................... 59

Figure 6.24 - PID velocity errors for 1 year (differing initial conditions) .........................59

Figure 6.25 - PID $\Delta \mathrm{v}$ profile for 1 year (differing initial conditions) ..................................6 60

Figure 6.26 - PID position errors for 1 year (worst case) .................................................. 61

Figure 6.27 - PID velocity errors for 1 year (worst case) .............................................. 62

Figure 6.28 - PID $\Delta v$ profile for 1 year (worst case) ..................................................... 62

Figure 7.1 - Simplified LQR block diagram ................................................................... 66 
Figure 7.2 - LQR position errors for 1 year (unperturbed) .......................................... 68

Figure 7.3 - LQR velocity errors for 1 year (unperturbed) …………………………....... 68

Figure 7.4 - LQR $\Delta v$ profile for 1 year (unperturbed) …………….................................. 69

Figure 7.5 - LQR position errors for 1 year (thruster misalignments) ............................ 70

Figure 7.6 - LQR velocity errors for 1 year (thruster misalignments) .............................. 71

Figure 7.7 - LQR $\Delta \mathrm{v}$ profile for 1 year (thruster misalignments) ................................... 71

Figure 7.8 - LQR position errors for 1 year (thruster bias).............................................. 73

Figure 7.9 - LQR velocity errors for 1 year (thruster bias).......................................... 73

Figure 7.10 - LQR $\Delta \mathrm{v}$ profile for 1 year (thruster bias)................................................. 74

Figure 7.11 - LQR position errors for 1 year (self-gravity) ............................................. 75

Figure 7.12 - LQR velocity errors for 1 year (self-gravity) .......................................... 76

Figure 7.13 - LQR $\Delta \mathrm{v}$ profile for 1 year (self-gravity) ……………………………....... 76

Figure 7.14 - LQR position errors for 1 year (Jupiter) ……………………………..... 78

Figure 7.15 - LQR velocity errors for 1 year (Jupiter) …………………………........ 78

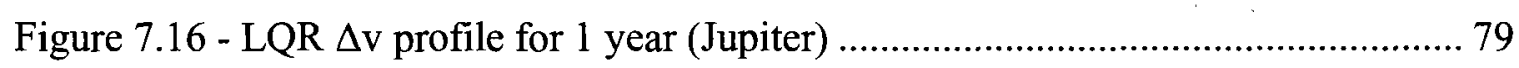

Figure 7.17 - LQR position errors for 1 year (solar radiation pressure) ............................ 80

Figure 7.18 - LQR velocity errors for 1 year (solar radiation pressure) .......................... 81

Figure 7.19 - LQR $\Delta \mathrm{v}$ profile for 1 year (solar radiation pressure) .................................. 81

Figure 7.20 - LQR position errors for 1 year (measurement noise)................................. 83

Figure 7.21 - LQR velocity errors for 1 year (measurement noise).................................. 83

Figure 7.22 - LQR $\Delta \mathrm{v}$ profile for 1 year (measurement noise)....................................... 84

Figure 7.23 - LQR position errors for 1 year (differing initial conditions) ...................... 85

Figure 7.24 - LQR velocity errors for 1 year (differing initial conditions) ...................... 86 
Figure 7.25 - LQR $\Delta v$ profile for 1 year (differing initial conditions)

Figure 7.26 - LQR position errors for 1 year (worst case).............................................. 88

Figure 7.27 - LQR velocity errors for 1 year (worst case),................................................ 88

Figure 7.28 - LQR $\Delta \mathrm{v}$ profile for 1 year (worst case)...................................................... 89

Figure 8.1 - $\mathrm{H}_{\infty}$ block diagram ................................................................................ 95

Figure 8.2 - $\mathrm{H}_{\infty}$ position errors for 1 year (unperturbed) .................................................. 96

Figure $8.3-\mathrm{H}_{\infty}$ velocity errors for 1 year (unperturbed) ................................................. 97

Figure $8.4-\mathrm{H}_{\infty} \Delta \mathrm{v}$ profile for 1 year (unperturbed) …………........................................ 97

Figure $8.5-\mathrm{H}_{\infty}$ position errors for 1 year (thruster misalignments) ................................. 99

Figure $8.6-\mathrm{H}_{\infty}$ velocity errors for 1 year (thruster misalignments) .............................. 100

Figure $8.7-\mathrm{H}_{\infty} \Delta \mathrm{v}$ profile for 1 year (thruster misalignments) .................................... 100

Figure $8.8-\mathrm{H}_{\infty}$ position errors for 1 year (thruster bias).............................................. 102

Figure $8.9-\mathrm{H}_{\infty}$ velocity errors for 1 year (thruster bias) ................................................. 102

Figure $8.10-\mathrm{H}_{\infty} \Delta \mathrm{v}$ profile for 1 year (thruster bias) …………........................................ 103

Figure $8.11-\mathrm{H}_{\infty}$ position errors for 1 year (self-gravity) ............................................. 104

Figure $8.12-\mathrm{H}_{\infty}$ velocity errors for 1 year (self-gravity) .............................................. 105

Figure $8.13-\mathrm{H}_{\infty} \Delta \mathrm{v}$ profile for 1 year (self-gravity) ………....................................... 105

Figure $8.14-\mathrm{H}_{\infty}$ position errors for 1 year (Jupiter) ....................................................... 107

Figure $8.15-\mathrm{H}_{\infty}$ velocity errors for 1 year (Jupiter)..................................................... 107

Figure $8.16-\mathrm{H}_{\infty} \Delta \mathrm{v}$ profile for 1 year (Jupiter) ...................................................... 108

Figure $8.17-\mathrm{H}_{\infty}$ position errors for 1 year (solar radiation pressure) ….......................... 109

Figure $8.18-\mathrm{H}_{\infty}$ velocity errors for 1 year (solar radiation pressure) ............................ 110

Figure $8.19-\mathrm{H}_{\infty} \Delta \mathrm{v}$ profile for 1 year (solar radiation pressure) .................................. 110 
Figure $8.20-\mathrm{H}_{\infty}$ position errors for 1 year (measurement noise)

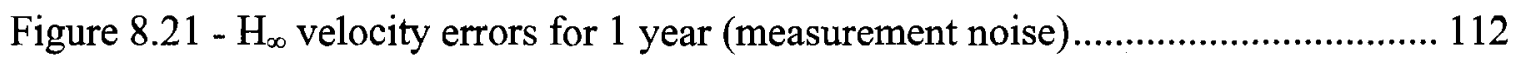

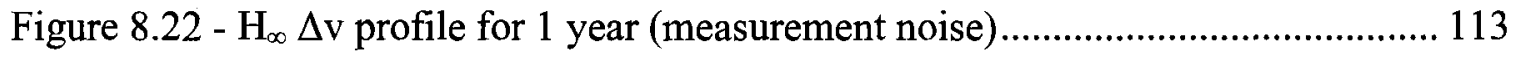

Figure $8.23-\mathrm{H}_{\curvearrowright}$ position errors for 1 year (differing initial conditions)..................... 114

Figure $8.24-\mathrm{H}_{\infty}$ velocity errors for 1 year (differing initial conditions)...................... 115

Figure $8.25-\mathrm{H}_{\infty} \Delta \mathrm{v}$ profile for 1 year (differing initial conditions)............................ 115

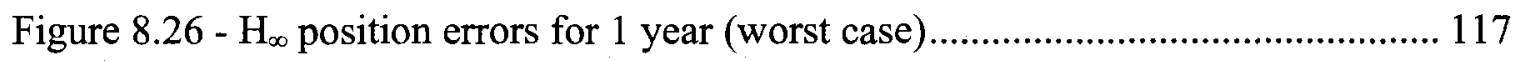

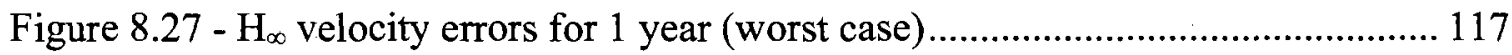

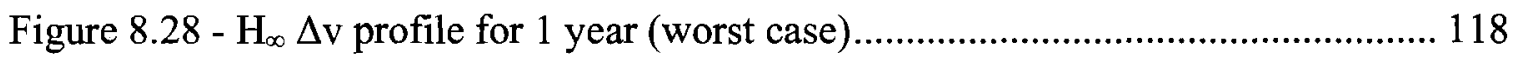

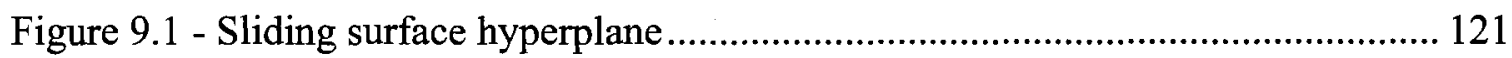

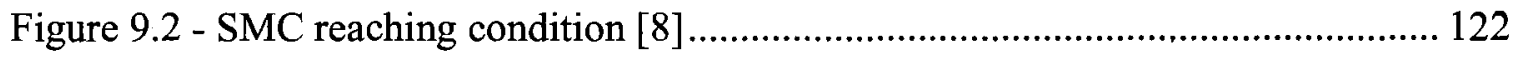

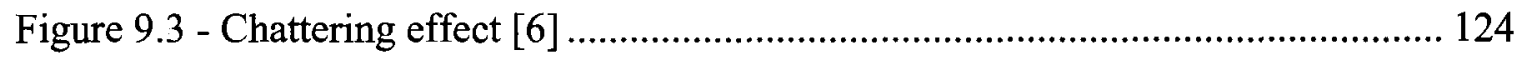

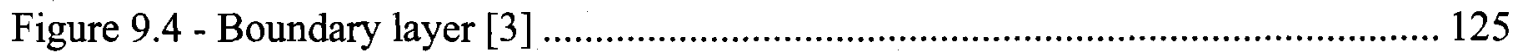

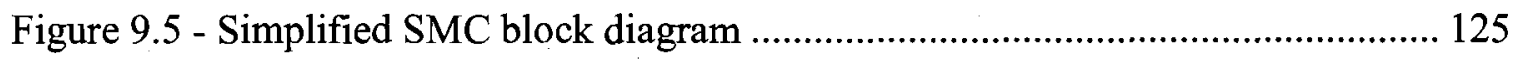

Figure 9.6 - SMC position errors for 1 year (unperturbed) ...................................... 127

Figure 9.7 - SMC velocity errors for 1 year (unperturbed).................................... 128

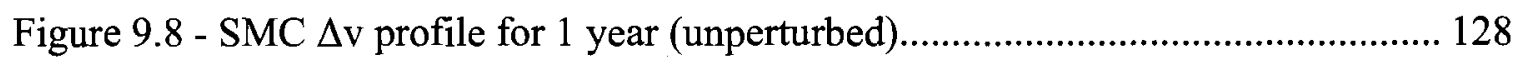

Figure 9.9 - SMC position errors for 1 year (thruster misalignments) ......................... 130

Figure 9.10 - SMC velocity errors for 1 year (thruster misalignments) ....................... 131

Figure 9.11 - SMC $\Delta v$ profile for 1 year (thruster misalignments) …....................... 131

Figure 9.12 - SMC position errors for 1 year (thruster bias) .................................. 133

Figure 9.13 - SMC velocity errors for 1 year (thruster bias) .................................. 133

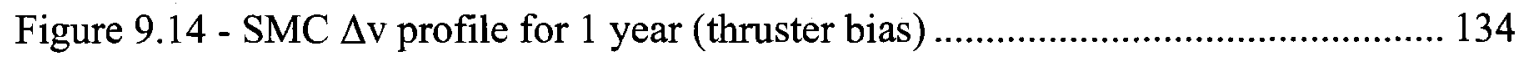


Figure 9.15 - SMC position errors for 1 year (self-gravity)

Figure 9.16 - SMC velocity errors for 1 year (self-gravity) .......................................... 136

Figure 9.17 - SMC $\Delta v$ profile for 1 year (self-gravity) ……………………………..... 136

Figure 9.18 - SMC position errors for 1 year (Jupiter) ................................................. 138

Figure 9.19 - SMC velocity errors for 1 year (Jupiter) …….......................................... 138

Figure 9.20 - SMC $\Delta \mathrm{v}$ profile for 1 year (Jupiter) ………………................................. 139

Figure 9.21 - SMC position errors for 1 year (solar radiation pressure).......................... 140

Figure 9.22 - SMC velocity errors for 1 year (solar radiation pressure)......................... 141

Figure 9.23 - SMC $\Delta \mathrm{v}$ profile for 1 year (solar radiation pressure)............................... 141

Figure 9.24 - SMC position errors for 1 year (measurement noise) ................................ 143

Figure 9.25 - SMC velocity errors for 1 year (measurement noise) ............................... 143

Figure 9.26 - SMC $\Delta \mathrm{v}$ profile for 1 year (measurement noise) ………………………... 144

Figure 9.27 - SMC position errors for 1 year (differing initial conditions) .................... 145

Figure 9.28 - SMC velocity errors for 1 year (differing initial conditions) ..................... 146

Figure 9.29 - SMC $\Delta \mathrm{v}$ profile for 1 year (differing initial conditions) ............................ 146

Figure 9.30 - SMC position errors for 1 year (worst case) .............................................. 148

Figure 9.31 - SMC velocity errors for 1 year (worst case) ............................................... 148

Figure 9.32 - SMC $\Delta \mathrm{v}$ profile for 1 year (worst case) .................................................... 149

Figure A1 - Overall system model.............................................................................. 163

Figure A2 - Nonlinear CR3BP equations of motion.................................................... 164

Figure A3 - Reference model.................................................................................. 166

Figure A4 - Thruster bias / misalignment .................................................................. 167

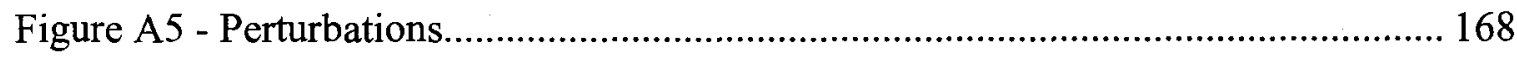




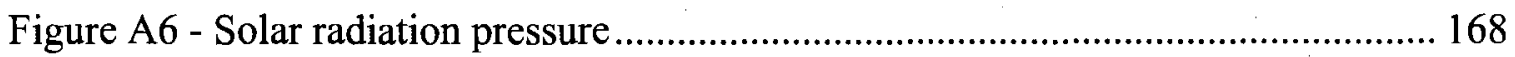

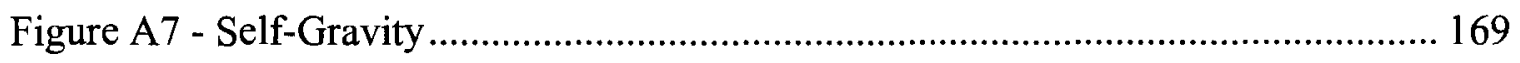

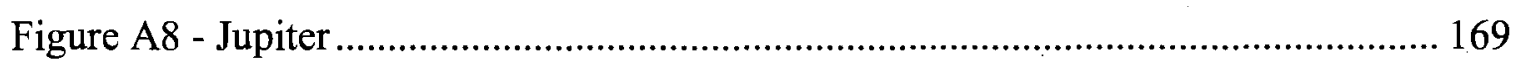

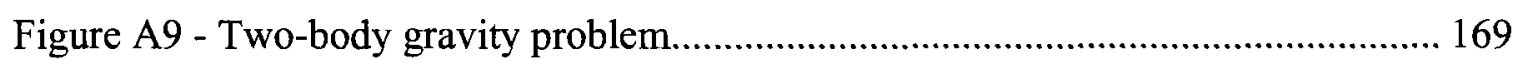

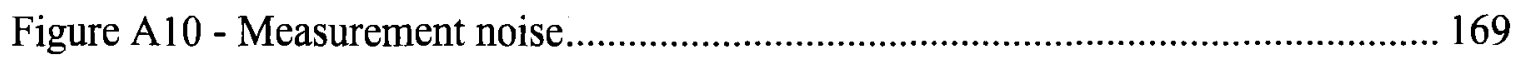

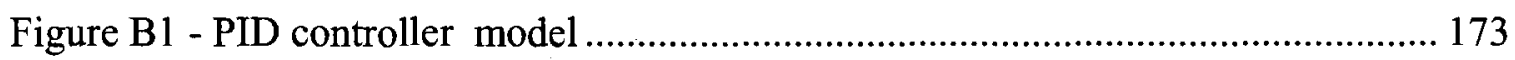

Figure C1 - LQR controller model......................................................................... 175

Figure D1 - $\mathrm{H}_{\infty}$ simulink model .......................................................................... 176

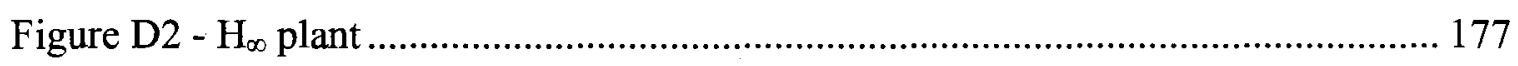

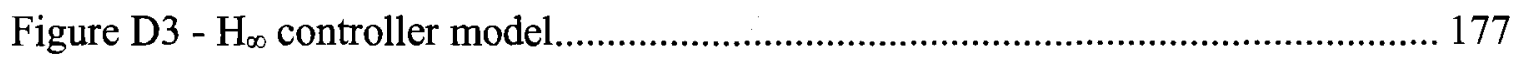

Figure E1 - SMC controller model ........................................................................ 179 


\section{LIST OF TABLES}

Table 2.1 - Mission error tolerances 15

Table 3.1 - Approximate locations of Earth/Moon-Sun libration points (in AU) 21

Table 6.1 - PID results for 1 year (unperturbed) ............................................................. 43

Table 6.2 - PID results for 1 year (thruster misalignments) ........................................... 46

Table 6.3 - PID results for 1 year (thruster bias) ………….............................................. 48

Table 6.4 - PID results for 1 year (self-gravity)........................................................... 51

Table 6.5 - PID results for 1 year (Jupiter) ………………………………………..... 53

Table 6.6 - PID results for 1 year (solar radiation pressure) (.......................................... 55

Table 6.7 - PID results for 1 year (measurement noise) ………………........................... 58

Table 6.8 - PID results for 1 year (differing initial conditions) ....................................... 60

Table 6.9 - PID results for 1 year (solar radiation pressure) ............................................ 63

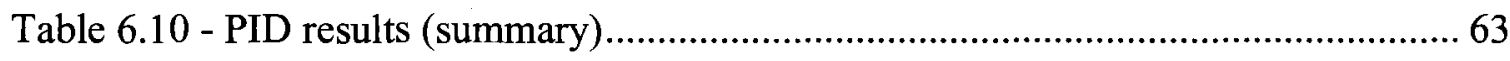

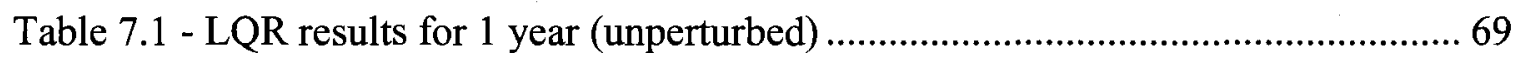

Table 7.2 - LQR results for 1 year (thruster misalignments) ……….............................. 72

Table 7.3 - LQR results for 1 year (thruster bias) …………………………………...... 74

Table 7.4 - LQR results for 1 year (self-gravity) .......................................................... 77

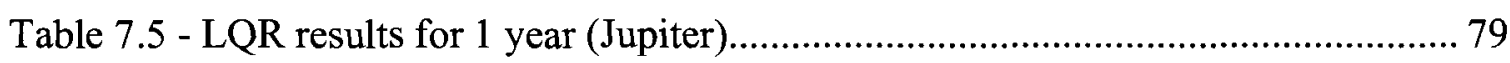

Table 7.6 - LQR results for 1 year (solar radiation pressure) …………........................... 82

Table 7.7 - LQR results for 1 year (measurement noise).................................................. 84

Table 7.8 - LQR results for 1 year (differing initial conditions) ....................................... 87 
Table 7.9 - LQR results for 1 year (solar radiation pressure) .......................................... 89

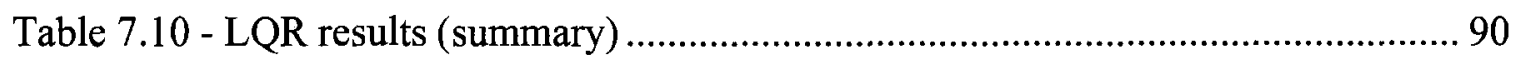

Table $8.1-\mathrm{H}_{\infty}$ results for 1 year (unperturbed) ………................................................ 98

Table $8.2-\mathrm{H}_{\infty}$ results for 1 year (thruster misalignments) ........................................... 101

Table $8.3-\mathrm{H}_{\infty}$ results for 1 year (thruster bias) ........................................................... 103

Table $8.4-\mathrm{H}_{\infty}$ results for 1 year (self-gravity) .......................................................... 106

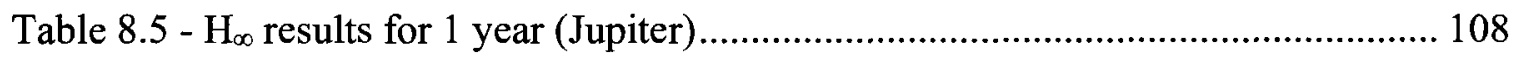

Table $8.6-\mathrm{H}_{\infty}$ results for 1 year (solar radiation pressure) .......................................... 111

Table $8.7-\mathrm{H}_{\infty}$ results for 1 year (measurement noise) ……....................................... 113

Table $8.8-\mathrm{H}_{\infty}$ results for 1 year (differing initial conditions)...................................... 116

Table $8.9-\mathrm{H}_{\infty}$ results for 1 year (solar radiation pressure) .......................................... 118

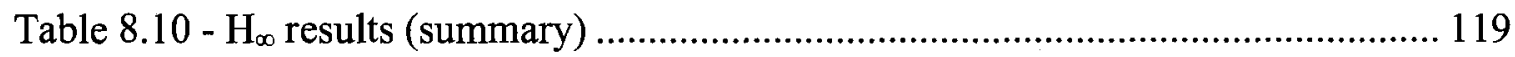

Table 9.1 - SMC results for 1 year (unperturbed) ........................................................ 129

Table 9.2 - SMC results for 1 year (thruster misalignments) ........................................ 132

Table 9.3 - SMC results for 1 year (thruster bias) …………........................................ 134

Table 9.4 - SMC results for 1 year (self-gravity)......................................................... 137

Table 9.5 - SMC results for 1 year (Jupiter) …………………................................... 139

Table 9.6 - SMC results for 1 year (solar radiation pressure) ........................................ 142

Table 9.7 - SMC results for 1 year (measurement noise) ……………………………... 144

Table 9.8 - SMC results for 1 year (differing initial conditions) .................................... 147

Table 9.9 - SMC results for 1 year (solar radiation pressure) ......................................... 149

Table 9.10 - SMC results (summary) ........................................................................ 150 
Table 10.1 - Unperturbed results (comparison) …………………………………….... 151

Table 10.2 - Measurement noise results (comparison) …………………..................... 152

Table 10.3 - Differing initial conditions results (comparison) ……………................... 153

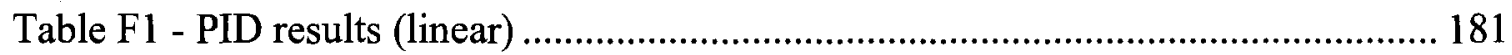

Table F2 - LQR results (linear)........................................................................... 181

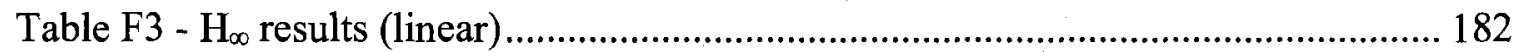

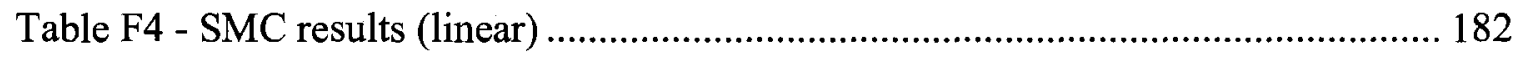




\begin{abstract}
A COMPARATIVE ANALYSIS OF CONTROL TECHNIQUES FOR FORMATION

FLYING SPACECRAFT IN AN EARTH/MOON-SUN L L $_{2}$-CENTERED LISSAJOUS
\end{abstract}

ORBIT

by

Michael Farrar

University of New Hampshire, September 2008

In this thesis, several control techniques are applied to an occulter satellite for a given formation flying mission. This research is in collaboration with the Flight Dynamics Analysis branch at the NASA Goddard Space Flight Center in Greenbelt, Maryland. The spacecraft is part of a leader-follower configuration which orbits about the Earth/Moon-Sun $\mathrm{L}_{2}$ libration point in a lissajous orbit. A controller is required to maintain a distance of $50,000 \mathrm{~km}$ between the occulter and the leader satellite in the radial direction with respect to the orbit. The occulter is allowed a tolerance range of $10 \mathrm{~m}$ within the "shadow" of the leader. In addition, the controller must also minimize the fuel usage $(\Delta v)$ needed to maintain the occulter's trajectory.

The spacecraft model follows the equations of motion defined by the circular restricted three body problem (CR3BP), where the primary bodies are defined to be the Earth/Moon system and the Sun. The dynamic model also incorporates thruster errors and misalignments, orbital sensor noise, environmental perturbations and disturbances, and additional modeling errors/uncertainties. 
The control techniques analyzed in this paper consist of several linear (PID, Linear Quadratic Regulator, and $\mathrm{H}_{\infty}$ ) and one nonlinear controller (Sliding Mode Control). All control techniques are compared based on the overall minimization of trajectory error and fuel usage, ease of implementation, and robustness against disturbances and perturbations such as solar radiation pressure and thruster misalignments.

The results of this research show that of the control techniques analyzed, the Linear Quadratic Regulator (LQR) and Sliding Mode Control (SMC) satisfy the mission requirements. While LQR uses less fuel to satisfy given mission requirements, the SMC would also be a suitable choice of control if a mission weighted accuracy over fuel usage. 


\section{CHAPTER 1}

\section{INTRODUCTION}

\subsection{Satellite Formation Flying}

A spacecraft formation is a group of two or more spacecraft that fly in a controlled configuration and operate together to complete a joint task for a given mission. Each mission requires the spacecraft to maintain a bounded distance to preserve communications with one another while still being capable of completing their individual tasks.

Several satellite formation configurations exist, such as a constellation, trailing, and cluster. A satellite constellation consists of multiple satellites orbiting in a maintained configuration. A leader / occulter formation is a two satellite system with an occulter (or follower satellite) whose orbit is defined by the leader. In a trailing formation, multiple satellites follow the same path maintaining a specified distance between each other. A satellite cluster consists of a group of satellites that orbit within a small space or cluster. In most every configuration, orbit and attitude require some form of control [10]. 
Formations are a useful way to avoid the complications of launching a large satellite. For example, a large satellite would not be able to be launched in a single piece, but would need to be launched in separate parts and then assembled in space. With the use of a satellite formation, multiple small satellites may be launched separately and once in orbit, the spacecraft transmit information to one another, avoiding the need for mid-space assembly.

The elimination of a large satellite also decreases the perturbation of some nonlinear disturbances. External disturbances, such as gravitational forces and solar radiation, increase with a larger mass or surface area, causing an increase in required fuel.

The spacecraft formation in this research is to orbit about an equilibrium point in space known as a libration or Lagrange point. Libration points exist within the scope of two large bodies, where if a third body of negligible mass is placed, it will remain stationary with respect to the two larger bodies. In every two-body system, five libration points exist in configuration seen in Figure 1.1. $\mathrm{L}_{1}$ and $\mathrm{L}_{2}$ are two points that are of extreme interest in the Earth/Moon-Sun system. At these locations, a spacecraft can analyze Earth, the Sun, and/or deep-space (to be explained in much further detail in Chapter 3). 


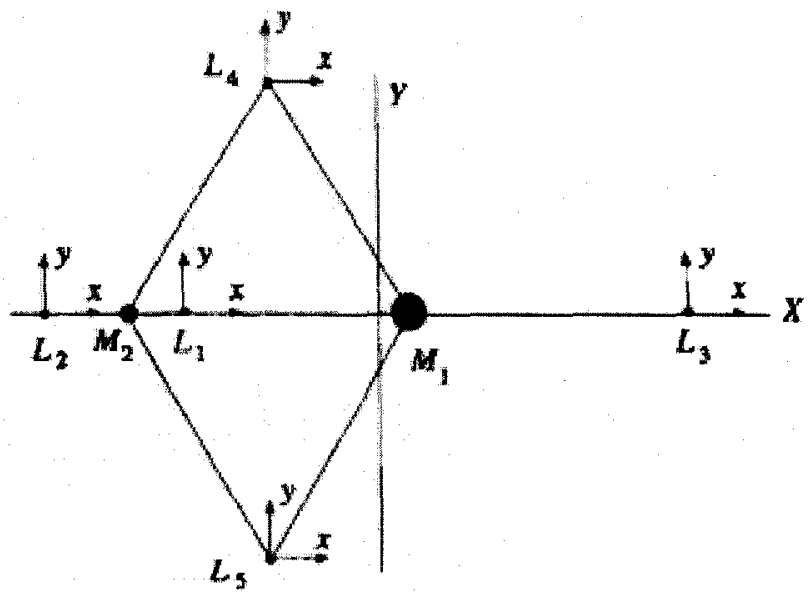

Figure 1.1 - Location of libration points

\subsection{Examples of Past, Current and Future Missions}

The Terrestrial Planet Finder (TPF) was a proposed project from NASA that would use interferometry to locate new planets. Interferometry is the use of multiple telescopic spacecrafts in a constellation that simulates a much larger telescope. TPF was to consist of a coronagraph to locate a large star which would then block out its light. With the light blocked, the remaining parts of the constellation would be able to take images of the dimmer lit bodies orbiting about the parent star. In early 2007, the project was indefinitely postponed [12].

The Solar Terrestrial Relations Observatory (STEREO) is a current NASA mission composed of identical spacecrafts in a trailing formation. The two spacecrafts were launched in October of 2006 into a geosynchronous orbit, remaining above a fixed location on Earth. The spacecraft formation has been given several missions, including 
the Sun Earth Connection Coronal and Heliospheric Investigation (SECCHI), In-situ Measurements of Particles and CME Transients (IMPACT), Plasma and Suprathermal Ion Composition (PLASTIC) and Stereo/Waves (SWAVES). All these missions have objectives that further the study of the Sun and its effects on Earth by recording threedimensional images of coronal mass ejections from the Sun,. STEREO will complete its mission in the second half of 2008 [8].

The Cluster mission is a group of four identical spacecrafts in tetra-hedral formation launched by ESA in 2000 . The main mission objective of Cluster is to analyze and capture three-dimensional images of the Earth's magnetosphere, a magnetic field around Earth that provides protection from solar wind. The four spacecrafts maintain distances that range from 100 to $10,000 \mathrm{~km}$, which travel about Earth in a polar orbit. The mission was scheduled for completion in 2003 , but has since been pushed back multiple times to a current date of December 2009 [11]. A mission with a similar scientific mission and spacecraft formation is currently being developed by NASA, called the Magnetospheric Multiscale (MMS) Mission [30].

The Micro-Arcsound X-ray Imaging Mission (MAXIM) is a proposed spacecraft constellation that would drastically improve upon that of current imaging spacecraft by a factor of ten million. The interferometry system is to be composed of a group of 25 spacecraft which would record images away from Earth, primarily black holes, and that must maintain accuracy on the micron level. MAXIM is to be placed in orbit about the $\mathrm{L}_{2}$ libration point [9]. 
The Gravity Recovery and Interior Laboratory (GRAIL) mission is a set of spacecraft flying in a tandem constellation about the moon. NASA currently plans to launch the formation in 2011, where by two satellites are to take $\mathrm{x}$-ray images of the moon to reveal subsurface structures and thermal history [13].

\subsection{Past Research}

In the complex environment of space, there is a great need for the control of a spacecraft due to, for example, the many perturbations and disturbances that act upon the spacecraft. The general area of spacecraft control is a well established field [1], [18], [19]. As technology and mission complexity increase, however, so does the need for a control design.

Two area of spacecraft control exist: attitude and orbit control. Attitude refers to the spacecraft's orientation while orbit refers to the spacecraft's position and translational motion. This research focuses on orbital control, requiring extensive knowledge of its dynamics [1], [18], [20].

There has been much research performed on spacecraft in the region of a libration point. Some studies have been performed by Marchand [14], Lo [21] and Wong [22] to investigate the dynamics and possibility of future missions in the libration region. Also, 
Dunham [23] and $\mathrm{Xu}$ [24] have investigated the use of control for station-keeping procedures while orbiting a libration point.

Formation flying is another major focus within spacecraft design. Berge [25], Li [26] and Lay [27] have investigated various formation techniques, while Folta [9], Scheeres [15], Marchand [16], Infield [28], and Vadali [29] have used control techniques to maintain spacecraft formations in the vicinity of libration points. Each of these works studies a specific set of satellites while testing one or two types of control techniques. The Linear Quadratic Regulator (LQR) is the most widely used controller, while the other control techniques presented in this research are used less often. The level of performance of various types of control vary from mission to mission. The purpose of this research is to present a comparative study of various controllers for formation flying spacecraft. Without loss of generality, this research will use the generic nonlinear equations of motion for the circular restricted three body problem for spacecraft orbiting the Earth/Moon-Sun $\mathrm{L}_{2}$ libration point for a given orbit trajectory, as specified by the Flight Dynamics Analysis Branch of the NASA Goddard Space Flight Center.

\subsection{Fuel Usage}

For a spacecraft formation to maintain a desired orbit within the nonlinear dynamics of space, active feedback control may be applied. Orbits are primarily maintained with the use of thrusters. The drawback of thrusters, however, is that fuel must be carried 
onboard the spacecraft to provide the energy needed for the thrusters. The added weight, in turn, requires more fuel to propel the additional inertia and also adds significantly to the cost of the launch and the overall mission. Once in orbit, the spacecraft has only a finite amount of fuel to maintain its orbit for the remainder of its mission. Therefore, a controller must be designed so that the thrusters use the minimum possible amount of fuel to remain "on course" throughout a spacecraft's entire mission. The fuel usage is measured in terms of changes in the spacecraft's velocity, $\Delta \mathrm{v}$ :

$$
\Delta v=\frac{1}{m_{\text {sat }}} \int_{t_{0}}^{t_{t}} u(t) d t
$$

where $u$ is the acceleration provided by the thrusters and $m_{s a t}$ is the assumed constant mass of the spacecraft.

\subsection{Thesis Objectives}

This research is in collaboration with the Flight Dynamics Analysis Branch at the NASA Goddard Space Flight Center through the direction of David Folta, Senior Aerospace Engineer. This thesis provides a comparative analysis for several control techniques used for spacecraft formation flying in the vicinity of the $\mathrm{L}_{2}$ libration point. The spacecraft formation in study is of the leader / occulter formation, orbiting $\mathrm{L}_{2}$ in a lissajous orbit for one year. The system is modeled under the equations of motion defined by the circular restricted three body problem. Four types of controllers are compared in this study. Each controller is designed to maintain the occulter at a $50,000 \mathrm{~km}$ distance from the leader 
with an accuracy of $10 \mathrm{~m}$. The control effort is used to maintain the occulter, while the leader is assumed to be perfectly controlled. In addition, perturbations from internal and external sources are simulated to test the robustness of each controller. Each of the controller's performance is analyzed based upon the amount of required control effort, system accuracy, robustness, and ease of implementation.

\subsection{Thesis Outline}

The following chapters are organized as follows:

- Chapter 2, Mission Description - A description of the mission objectives, including the determination of the lissajous orbit that the spacecrafts follow, are provided. Potential perturbations and disturbances are also discussed.

- Chapter 3, Circular Restricted Three Body Problem - The nonlinear equations of motion for the occulter satellite are derived and linearized. The definition and derivations of the Earth/Moon-Sun libration points are provided along with the derivation of the lissajous orbit.

- Chapter 4, Disturbances and Perturbations - Analyses of chosen disturbances and perturbations are provided.

- Chapter 5, Control Techniques - A brief description of various control techniques are discussed. 
- Chapters 6-9, PID, Linear Quadratic Regulator, $\mathrm{H}_{\infty}$, and Sliding Mode Control Each controller is defined with supporting equations and analyses. Results for all investigated disturbances are provided with discussions.

- Chapter 10, Conclusion - General remarks are provided regarding all controllers are compared to each other, followed by recommended future work and final remarks. 


\section{CHAPTER 2}

\section{MISSION DESCRIPTION}

\subsection{Lissajous Orbit}

Both the leader and occulter spacecrafts orbit about the Earth/Moon-Sun $\mathrm{L}_{2}$ libration point ${ }^{1}$ in a lissajous orbit. A lissajous orbit is a quasi-periodic orbit, meaning that a phase shift exists which moves the spacecraft so that it does not return to its starting position, seen in Figure 2.1. After an increasing number of orbital periods, the orbit diverges from, but eventually approaches its original orbit to form a closed path, as shown in Figure 2.2.

\footnotetext{
${ }^{1}$ A libration point exists in two body system, where all gravitational forces and accelerations negate each other, causing a third body to remain stationary with respect to the two bodies. This is explained in depth in Chapter 3.
} 


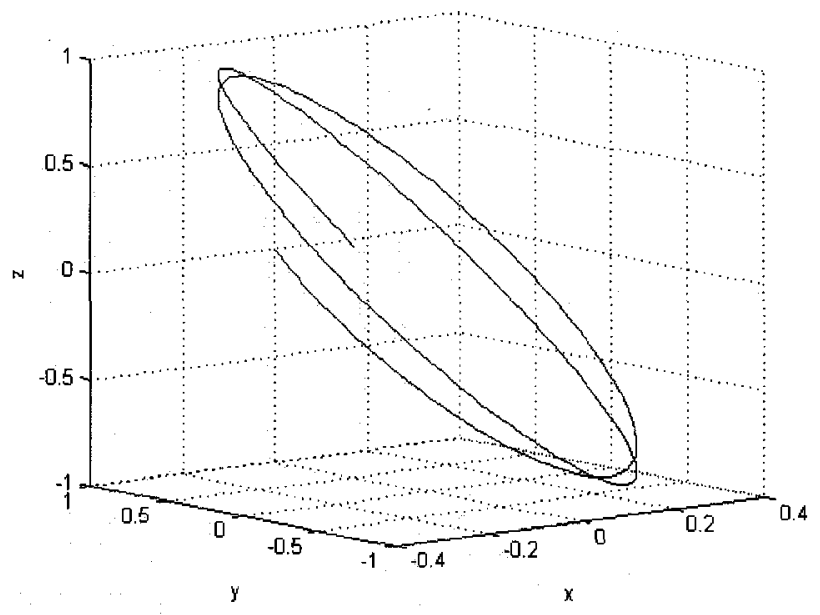

Figure 2.1 - Lissajous orbit for one year

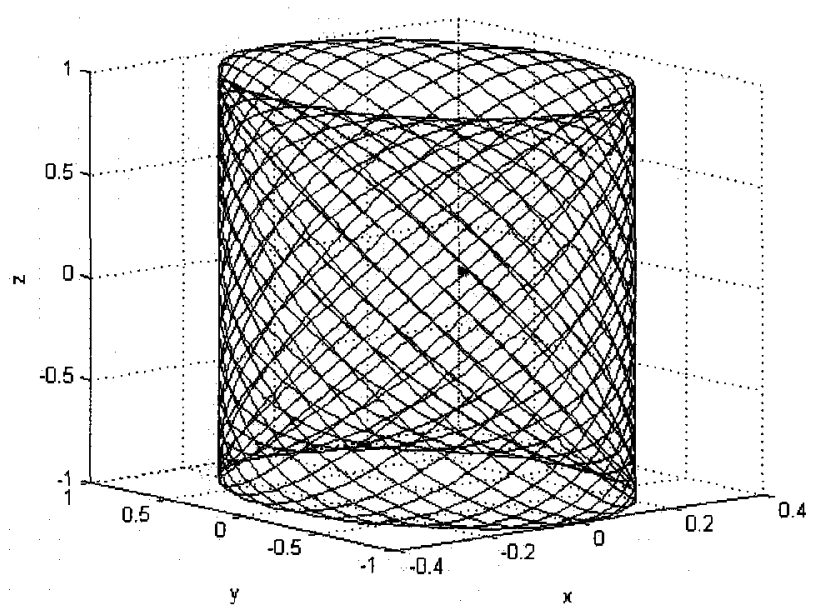

Figure 2.2 - Lissajous orbit for 15 years

A lissajous orbit is quasi-periodic due to its oscillatory modes. An orbit has three oscillatory modes, one in each basis direction. Each oscillatory mode defines the amount of phase shift that occurs. In the case of a closed or halo orbit, all three oscillatory modes are equal so the orbit will witness an equal phase shift in all directions, thus closing the orbit in one rotation. A lissajous orbit has two equal modes, referred to as the frequency of the in-plane oscillatory modes. These modes are usually in the $x$ and the $y$ directions. 
The third is an unequal mode in the $z$ direction and is known as the frequency of the outof-plane oscillatory mode.

Spacecraft orbits can be grouped in two categories, natural and non-natural. A natural orbit is a path on which a spacecraft can travel without the need for control. A nonnatural orbit, on the other hand, needs some form of control to maintain the orbit. A natural lissajous orbit can be found by analyzing the linearized equations of motion for the circular restricted three body problem (CR3BP) (shown in section 3.6). Although the orbit is natural for the linearized system, the original nonlinear system is non-natural. Therefore, the real dynamics corresponding to the CR3BP require control to maintain a desired lissajous orbit.

\section{$\underline{\text { 2.2 Leader Orbit }}$}

As stated earlier the leader spacecraft orbits about $\mathrm{L}_{2}$ in a lissajous trajectory. A lissajous orbit, however, is only stable when considering to the linearized format of the CR3BP equations of motion. Simulation results show, however, that the lissajous orbit is not completely natural due to the existing nonlinearities of the CR3BP dynamics and to its sensitivity to varying initial conditions. The leader orbit in this research is given to be a linearized form of a chosen lissajous orbit with specified initial conditions. The resulting orbit, seen in Figure 2.3, is completely natural, as expected. 

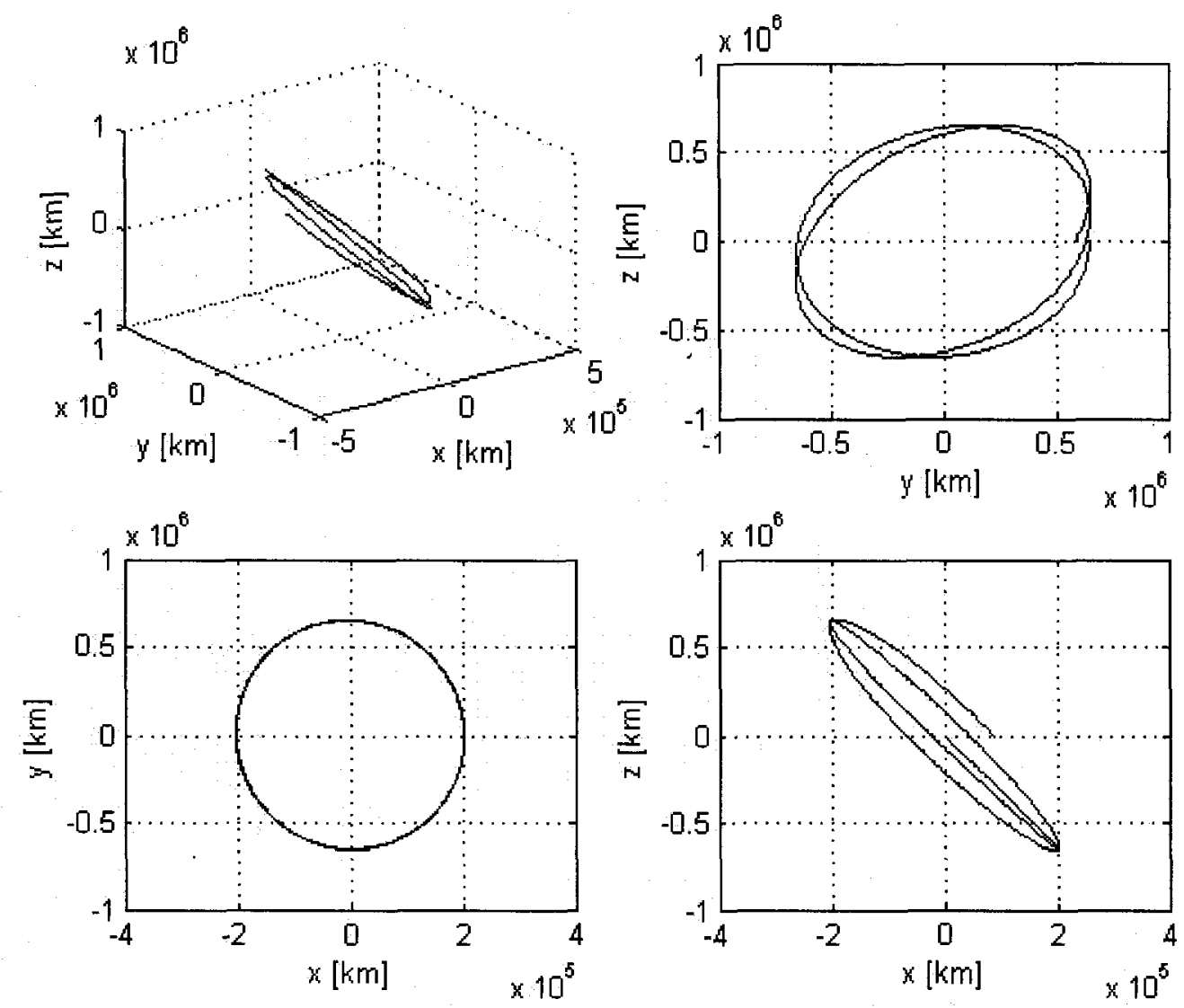

Figure 2.3 - Leader orbit for one year

\subsection{Occulter Path}

The occulter (follower) is to follow the leader's orbit at a distance of $50,000 \mathrm{~km}$ radially outwards from the Earth-Sun $\mathrm{L}_{2}$ point. The leader and occulter's orbits in relation to Earth can be see in Figure 2.4. Here, however, the full nonlinear dynamics of the CR3BP are assumed. To maintain this orbit, then, an active feedback controller is applied in this research. 


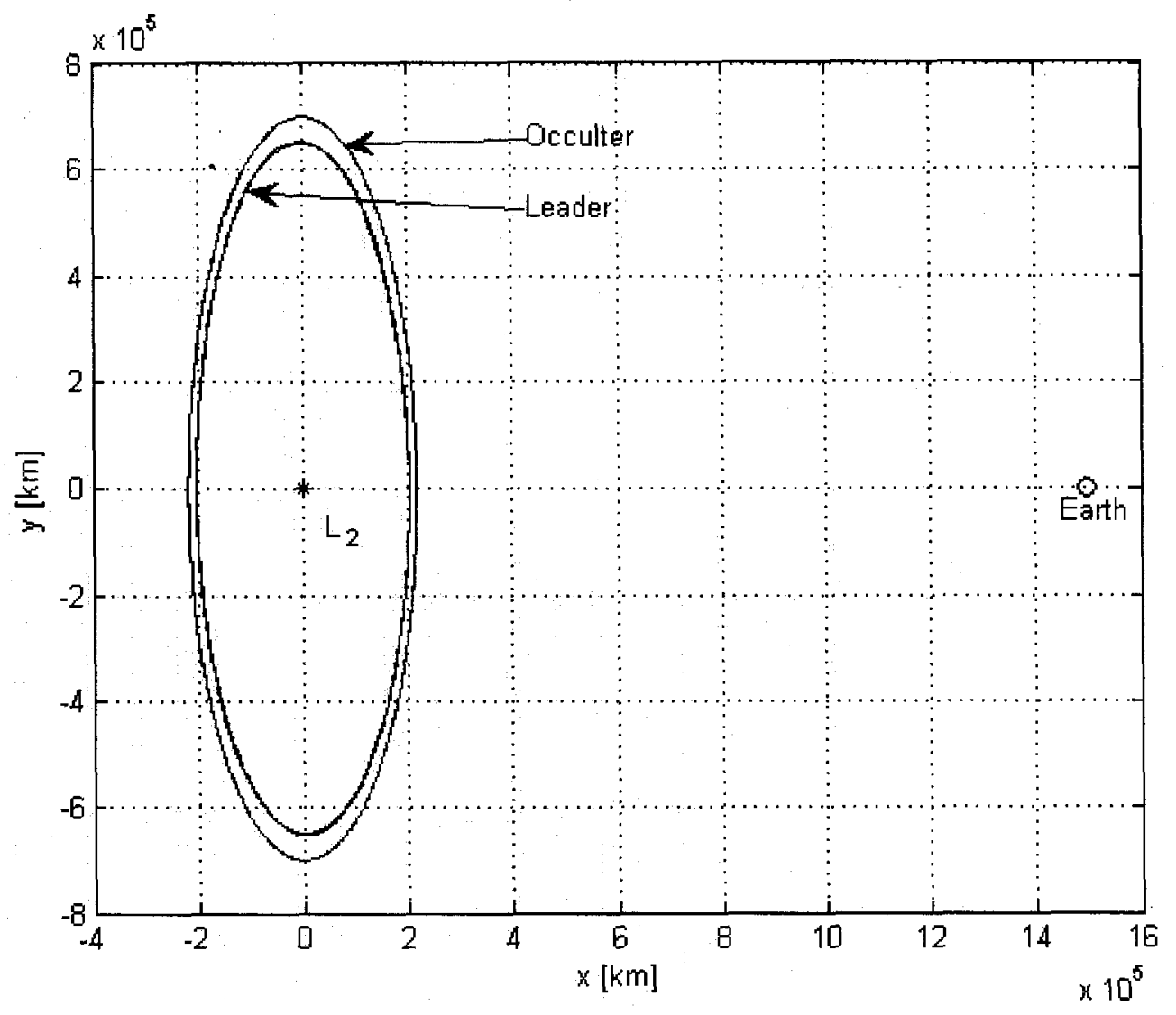

Figure 2.4 - Leader and occulter orbits with respect to Earth

\subsection{Mission Requirements}

The mission in study requires extreme accuracy between the two spacecrafts. The leader spacecraft is assumed to be perfectly controlled. Therefore, the responsibility of maintaining the required accuracy falls completely on the occulter. The occulter must maintain its $50,000 \mathrm{~km}$ distance from the leader but is given a $\pm 10 \mathrm{~m}$ tolerance in each of the $\mathrm{x}-, \mathrm{y}-$, and $\mathrm{z}$-directions. More importantly, however, the occulter must use as little 
fuel as possible. As explained earlier fuel is a strict commodity and its use must be minimized while concurrently maintaining a prescribed error tolerance.

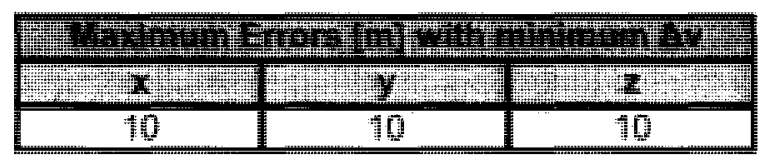

Table 2.1 - Mission error tolerances

\subsection{Perturbations}

In addition to the precise mission requirements, the robustness of the system must also be tested against internal and external perturbations. The following perturbations are investigated:

- solar radiation pressure

- self-gravity between the two spacecraft

- gravity effects of Jupiter

- thruster misalignments and errors

- thruster bias

- measurement noise

- differing initial conditions (i.e. not starting at the desired initial position)

Each perturbation will be explained more in depth in their respective sections in Chapter 4. 
Other perturbations that could be an issue but are not considered in this study are:

- Earth's eccentricity

- separate gravitational effects of the Moon

- solar Wind

- Earth's magnetoshphere

These perturbations, although important, are not within the scope of this work and are left as future work. 


\section{CHAPTER 3}

\section{THE CIRCULAR RESTRICTED THREE BODY PROBLEM (CR3BP)}

\section{$\underline{\text { 3.1 Introduction }}$}

The circular restricted three body problem (CR3BP) was first formulated by Euler in 1772 [1]. Since its creation there has been no closed-form solution found. Numerical techniques, however, can be used to produce solutions to this problem.

The CR3BP consists of an infinitesimal mass, $m$, in the gravitational field of two large bodies, $M_{1}$ and $M_{2}$, known as the primaries. The smaller, third body is assumed to be of negligible mass with respect to the primaries. In this application the small mass $m$ represents the spacecraft, while $M_{1}$ represents the Sun and $M_{2}$ represents the combined mass of the Earth and the Moon. Here the Earth and the Moon are assumed as a single entity to simplify calculations. The barycenter of the Earth/Moon-Sun system lies between the Earth and the Moon. The proximity of the Moon to the barycenter and the size of its orbit results in a distance to the $\mathrm{L}_{2}$ that does not greatly change and can be assumed to produce gravitational force in the same direction as Earth. 


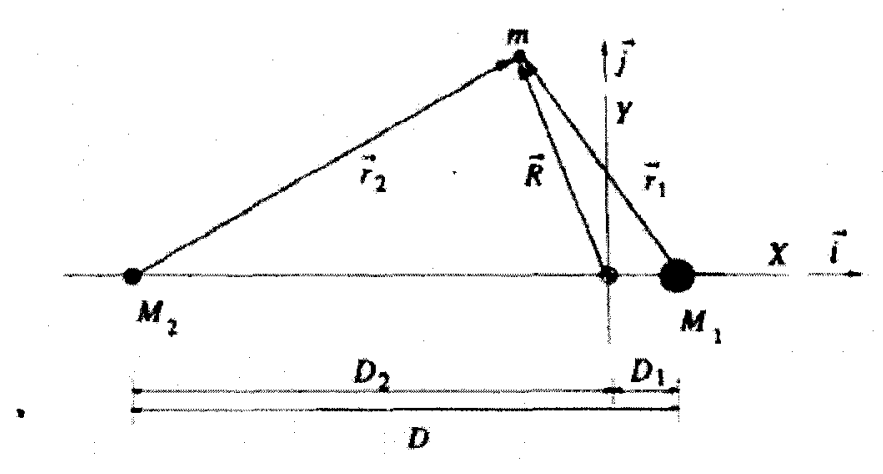

Figure 3.1 - Circular restricted three body problem [1]

Figure 1 shows the circular restricted three body problem. The origin of the system is placed at the center of gravity of the primaries, known as the barycenter. $D_{1}$ and $D_{2}$ denote the respective distances from $M_{1}$ and $M_{2}$ to the barycenter. The primaries rotate about their barycenter with a constant rotational velocity, $n$, such that

$$
n=\sqrt{G\left(M_{1}+M_{2}\right) / D^{3}}
$$

where $D$ is the distance between the two primaries. In the case of the Earth/Moon-Sun system, $D$ is one astronomical unit $(A U)$. The reference frame is defined to be rotating with a rate of $n \vec{k}$ to ensure that the primaries maintain a constant position with respect to the origin. (Here $\vec{k}$ completes the $\vec{i}, \vec{j}, \vec{k}$, orthogonal triad and extends above the 2-D plane in figure 3.1.)

The third body has distances $\mathrm{r}_{1}$ and $\mathrm{r}_{2}$ from $M_{1}$ and $M_{2}$, respectively, and a distance, $\vec{R}$, from the barycenter, such that

$$
\vec{R}=X \vec{i}+Y \vec{j}+Z \vec{k}
$$

where $\{\vec{i}, \vec{j}, \vec{k}\}$ are the basis vectors for the rotating reference frame. 


\subsection{Equations of Motion}

The spacecraft's inertial acceleration, $\ddot{\vec{R}}$, may be obtained by twice differentiating $\vec{R}$ from Equation (3.2) with respect time, yielding

$$
\ddot{\vec{R}}=\left(\ddot{X}-2 n \dot{Y}-n^{2} X\right) \vec{i}+\left(\ddot{Y}+2 n \dot{X}-n^{2} Y\right) \vec{j}+\ddot{Z} \vec{k}
$$

The terms with factor $2 n$ represent coriolis acceleration, while those terms with factor $n^{2}$ represent centrifugal acceleration.

Using the two-body gravitational formula, an additional equation for inertial acceleration can be written as

$$
m \ddot{R}=-\frac{G M_{1} m}{r_{1}^{3}} \vec{r}_{1}-\frac{G M_{2} m}{r_{2}^{3}} \vec{r}_{2}
$$

where $r_{1}=\left|\vec{r}_{1}\right|, r_{2}=\left|\vec{r}_{2}\right|$, and

$$
\begin{aligned}
& \vec{r}_{1}=-D_{1} \vec{i}+\vec{R}=\left(X-D_{1}\right) \vec{i}+\overrightarrow{Y j}+Z \vec{k} \\
& \vec{r}_{2}=D_{2} \vec{i}+\vec{R}=\left(X+D_{2}\right) \vec{i}+Y \vec{j}+Z \vec{k}
\end{aligned}
$$

The gravitational parameter, $\mu$, is defined as

$$
\mu=G M
$$

Equation (3.4) then can be rewritten as

$$
\ddot{\vec{R}}=-\frac{\mu_{1}}{r_{1}{ }^{3}} \vec{r}_{1}-\frac{\mu_{2}}{r_{2}{ }^{3}} \vec{r}_{2}
$$

Combining Equations (3.3) and (3.7) and grouping terms in the same unit directions, the equations of motion for the spacecraft become 


$$
\begin{gathered}
\ddot{X}-2 n \dot{Y}-n^{2} X=-\frac{\mu_{1}\left(X-D_{1}\right)}{r_{1}^{3}}-\frac{\mu_{2}\left(X+D_{2}\right)}{r_{2}{ }^{3}} \\
\ddot{Y}+2 n \dot{X}-n^{2} Y=-\frac{\mu_{1} Y}{r_{1}^{3}}-\frac{\mu_{2} Y}{r_{1}^{3}} \\
\ddot{Z}=-\frac{\mu_{1} Z}{r_{1}^{3}}-\frac{\mu_{2} Z}{r_{2}^{3}}
\end{gathered}
$$

Following the simplification process in [1], Equations (3.8) through (3.10) can be represented as

$$
\begin{gathered}
\ddot{X}-2 n \dot{Y}=\frac{\partial U}{\partial X} \\
\ddot{Y}+2 n \dot{X}=\frac{\partial U}{\partial Y} \\
\ddot{Z}=\frac{\partial U}{\partial Z}
\end{gathered}
$$

where $\mathrm{U}$ is referred to as the psuedopotential defined by

$$
U=\frac{1}{2} n^{2}\left(X^{2}+Y^{2}\right)+\frac{\mu_{1}}{r_{1}}+\frac{\mu_{2}}{r_{2}}
$$

\subsection{Libration Points}

The circular restricted three body problem contains some useful properties. Among them, the most significant is the existence of five stability points. These points are known as libration or Lagrange points. At these points, the gravitational forces from the primaries and the coriolis and centrifugal accelerations of the third smaller body negate 
each other. Thus, the time derivatives of the third body's position are equal to zero, allowing the mass to theoretically remain in a constant position with respect to the primaries.

If the time derivates of (3.8) through (3.10) are set to zero, five solutions can be calculated. These are shown in Table 3.1 and Figure 3.2.

\begin{tabular}{|c|c|c|c|}
\hline Libration Point & $\mathbf{X}$ & $\mathbf{Y}$ & $\mathbf{Z}$ \\
\hline $\mathrm{L}_{1}$ & -0.99 & 0 & 0 \\
\hline $\mathrm{L}_{2}$ & -1.01 & 0 & 0 \\
\hline $\mathrm{L}_{3}$ & 1 & 0 & 0 \\
\hline $\mathrm{L}_{4}$ & -0.5 & 0.87 & 0 \\
\hline $\mathrm{L}_{5}$ & -0.5 & -0.87 & 0 \\
\hline
\end{tabular}

Table 3.1 - Approximate locations of Earth/Moon-Sun libration points (in AU)

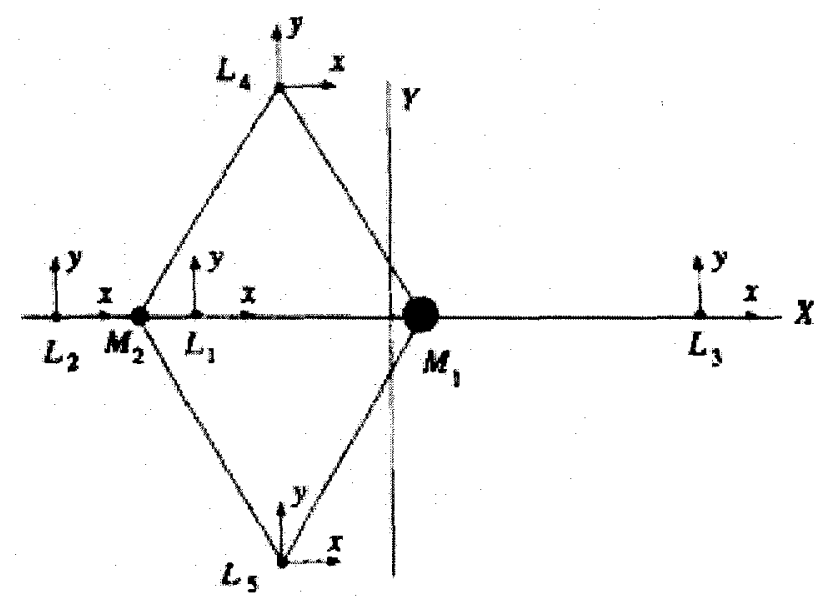

Figure 3.2 - Location of Earth/Moon-Sun libration points (not to scale), where $M_{1}$ represents the Sun and $M_{2}$ represents the Earth/Moon system [1]

There exists three collinear libration points $\left(\mathrm{L}_{1}, \mathrm{~L}_{2}\right.$, and $\left.\mathrm{L}_{3}\right)$ that are, in turn, collinear with the primaries. The other two libration points $\left(\mathrm{L}_{4}\right.$ and $\left.\mathrm{L}_{5}\right)$ are known as equilateral libration points, since they form equilateral triangles with the two primaries. The 
position of the points change in accordance to the primaries, but there are always three collinear and two equilateral libration points in the same arrangement.

\subsection{Linearized Equations of Motion}

Linearizing Equations (3.8) through (3.10) can be very useful in the analysis of a control system. Many controllers for nonlinear systems are based upon results of its linear counterpart. In [1], the linear forms of Equations (3.11) through (3.13) when linearized about a libration point are given, respectively, as

$$
\begin{gathered}
\ddot{x}-2 n \dot{y}-U_{x x} x=0 \\
\ddot{y}+2 n \dot{x}-U_{y y} y=0 \\
\ddot{z}-U_{z z} z=0
\end{gathered}
$$

where,

$$
\begin{aligned}
& U_{x x}=\left.\frac{\partial^{2} U}{\partial x^{2}}\right|_{L} \\
& U_{y y}=\left.\frac{\partial^{2} U}{\partial y^{2}}\right|_{L} \\
& U_{z z}=\left.\frac{\partial^{2} U}{\partial z^{2}}\right|_{L}
\end{aligned}
$$

and where $\mathrm{L}$ represents the chosen libration point.

Equations (3.15) through (3.17), then, can be rewritten in state-space form as follows:

$$
\dot{\mathbf{x}}=\mathbf{A x}
$$


where state $\mathbf{x}$ is

$$
\mathbf{x}=\left[\begin{array}{c}
x \\
y \\
z \\
\dot{x} \\
\dot{y} \\
\dot{z}
\end{array}\right]
$$

and

$$
\mathbf{A}=\left[\begin{array}{cccccc}
0 & 0 & 0 & 1 & 0 & 0 \\
0 & 0 & 0 & 0 & 1 & 0 \\
0 & 0 & 0 & 0 & 0 & 1 \\
U_{x x} & 0 & 0 & 0 & 2 n & 0 \\
0 & U_{y y} & 0 & -2 n & 0 & 0 \\
0 & 0 & U_{z z} & 0 & 0 & 0
\end{array}\right]
$$

Eigenvalue analysis can be performed on $\mathbf{A}$ for each Earth/Moon-Sun libration point. Doing so shows that the equilateral libration points have two negative real poles and four purely imaginary poles, while the collinear points have one negative and one positive real poles and four purely imaginary poles [1]. In other words, the equilateral libration points are globally stable, while the collinear libration points are semi-stable. That is, if placed at a collinear point, an object would remain stable for only a finite amount of time. 


\subsection{Translating the Origin}

The satellite in study is assumed to be orbiting about the $\mathrm{L}_{2}$ point. Therefore, the origin of the reference is translated to the $\mathrm{L}_{2}$ point to simplify error and distance calculations in post-processing. To do so, a new set of coordinates is defined:

$$
\begin{gathered}
\vec{x}=\vec{X}_{0}+\vec{X} \\
\vec{y}=\vec{Y} \\
\vec{z}=\vec{Z}
\end{gathered}
$$

where $x$ is the new $\mathrm{x}$-coordinate of the satellite, $X_{0}$ is the distance from $\mathrm{L}_{2}$ to the barycenter, and $X$ is the distance from the barycenter to satellite (note that $X$ is considered to be in the "negative $\vec{i}$ " direction) [2]. The new coordinate frame is shown in Figure 3.3.

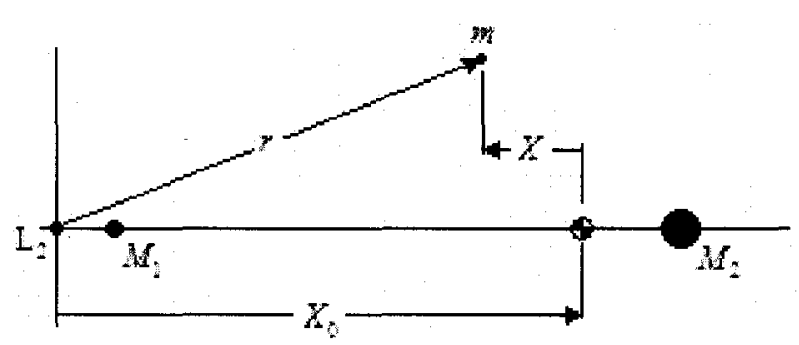

Figure 3.3 - Reference frame with translated origin

The new position vector of the satellite becomes

$$
\vec{r}=\left(X_{0}+X\right) \vec{i}+Y \vec{j}+Z \vec{k}
$$

or

$$
\vec{r}=x \vec{i}+y \vec{j}+z \vec{k}
$$

The linear and nonlinear equations of motion are updated by using the relations in Equations (3.22). 


\subsection{Lissajous Orbit}

As referenced in Chapter 2, the leader and the follower satellite orbit about $\mathrm{L}_{2}$ in a lissajous orbit. The lissajous orbit is derived based on the linearized equations of motion in Equation (3.19). The in-plane, $\omega_{x y}$, and out-of-plane, $\omega_{z}$, oscillatory modes are the imaginary eigenvalues of Equation (3.21) [1].

$$
\begin{aligned}
& \lambda_{3,4}= \pm j \omega_{x y} \\
& \lambda_{5,6}= \pm j \omega_{z}
\end{aligned}
$$

The appropriate choice of correct initial conditions, $\dot{\mathbf{x}}(0)$, allows for a solution of Equation (3.19), which contains only the oscillatory modes. This results in a quasiperiodic orbit that repeats itself over time. The required initial conditions for this quasiperiodic orbit are

$$
\begin{gathered}
\dot{x}(0)=\left(\omega_{x y} / k\right) y(0) \\
\dot{y}(0)=-k \omega_{x y} x(0)
\end{gathered}
$$

where

$$
k=\frac{\omega_{x y}^{2}+U_{x x}}{2 n \omega_{x y}}
$$

Equations (3.26) and (3.25) yield the following solutions to Equation (3.19):

$$
\begin{gathered}
x(t)=x(0) \cos \omega_{x y} t+(1 / k) y(0) \sin \omega_{x y} t \\
y(t)=y(0) \cos \omega_{x y} t-k x(0) \sin \omega_{x y} t \\
z(t)=z(0) \cos \omega_{z} t+\left[\dot{z}(0) / \omega_{z}\right] \sin \omega_{z} t
\end{gathered}
$$

The initial conditions, $x(0), y(0), z(0)$, and $\dot{z}(0)$, designate the size of the orbit. Choosing the initial conditions such that 


$$
\begin{aligned}
& x(0)=0 \\
& y(0)=7 \cdot 10^{8} \mathrm{~m} \\
& z(0)=0 \\
& \dot{z}(0)=-y(0) \omega_{z}
\end{aligned}
$$

yields the orbit in Figure 3.4. The orbit is in the rotating reference with its origin at $\mathrm{L}_{2}$. Figure 3.5 shows the orbit with relation to the Earth/Moon system. The star represents the $\mathrm{L}_{2}$ point while the circle represents the Earth/Moon system. Figures 3.6 and 3.7 show the orbit with respect to time. The resulting orbit is beneficial to $L_{2}$ missions since the satellite formation will be constantly seen by Earth, thus, eliminating the need for a relay satellite to transmit information if the satellite were to be out of sight/range.
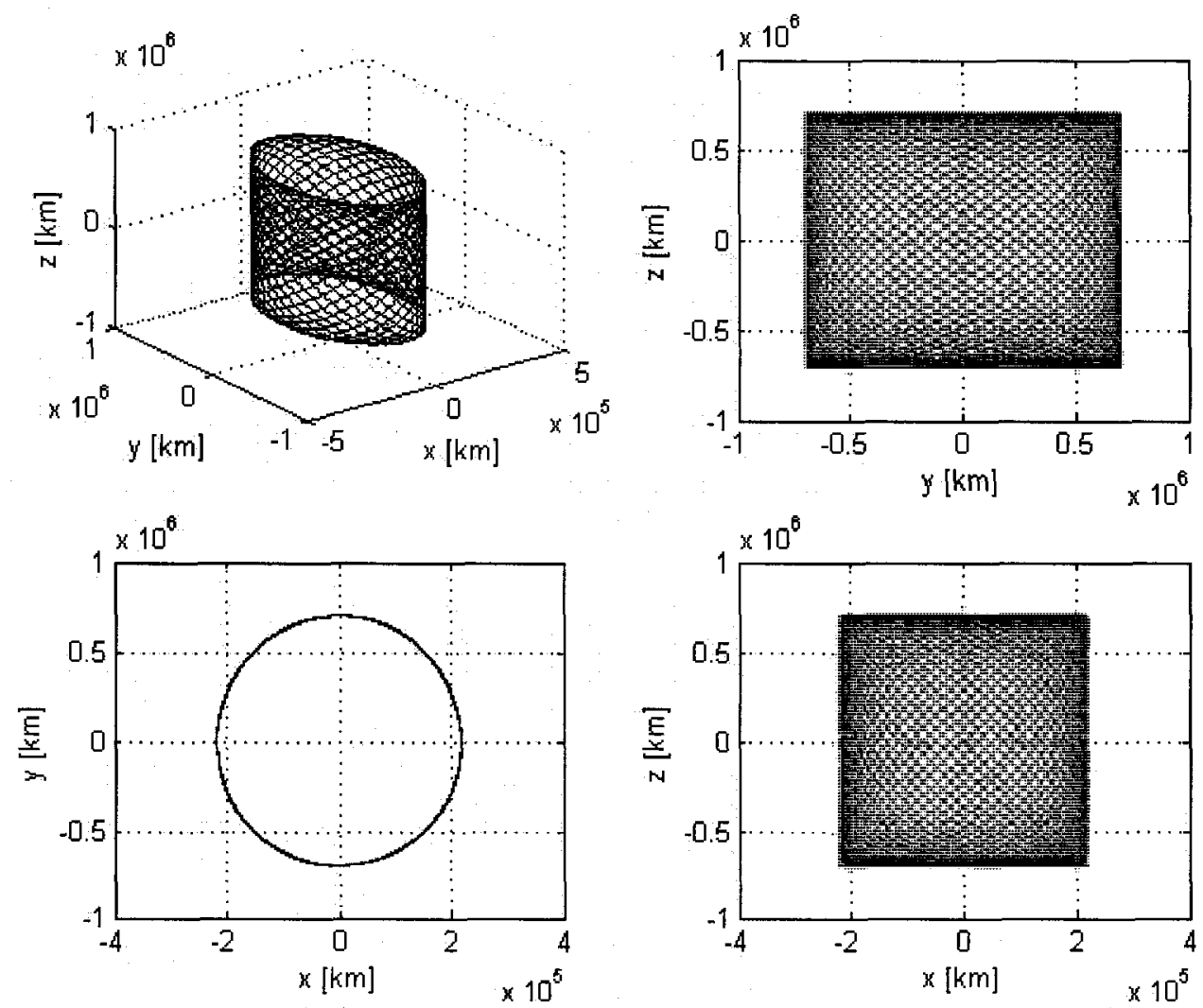

Figure 3.4 - Lissajous orbit 


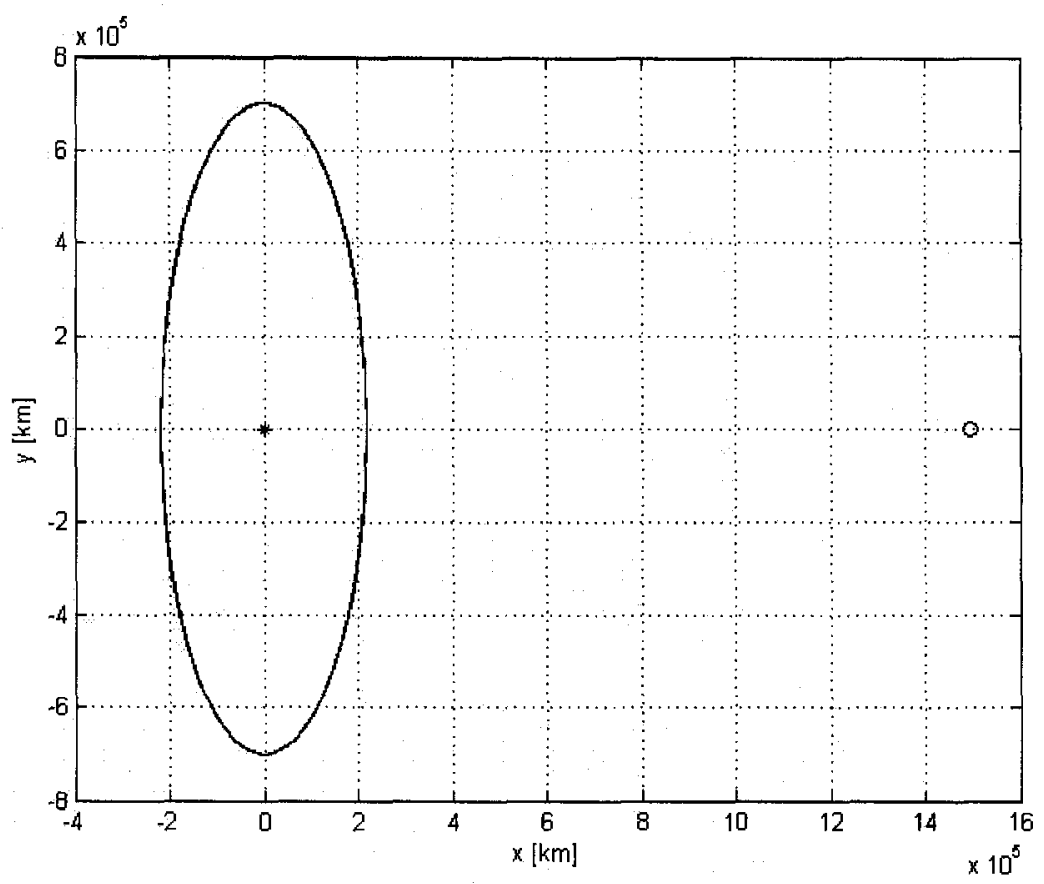

Figure 3.5 - Lissajous orbit with respect to the Earth/Moon system
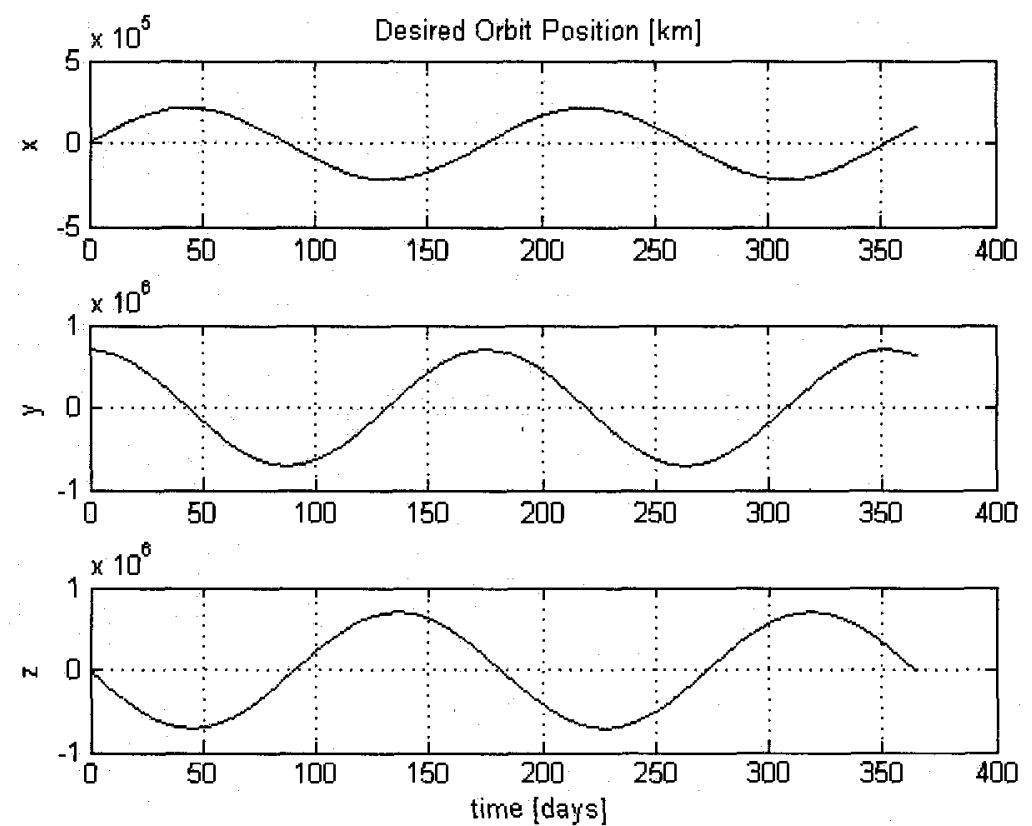

Figure 3.6 - Desired position 

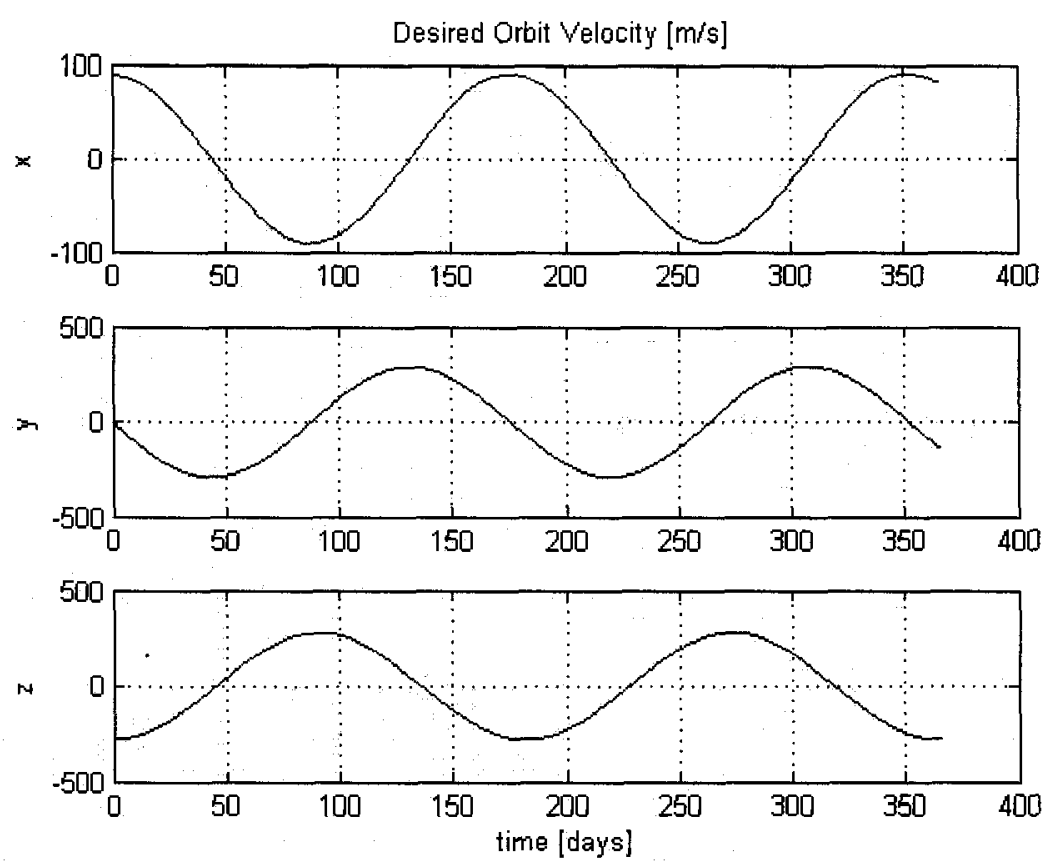

Figure 3.7 - Desired velocities 


\section{CHAPTER 4}

\section{DISTURBANCES AND PERTURBATIONS}

\subsection{Thruster Errors}

This section discusses disturbances / perturbations caused by thruster malfunctions: misalignments and bias errors.

\subsubsection{Thruster Misalignments}

The spacecraft's three thrusters may be misaligned by up to a tenth of a degree about each axis. To model this mathematically, the control effort is passed through a thruster misalignment gain matrix, TMM, such that

$$
\mathbf{T M M}=\left[\begin{array}{ccc}
\cos \theta \cos \theta & \cos \theta \sin \theta & \sin \theta \\
\cos \theta \sin \theta & \cos \theta \cos \theta & \sin \theta \\
\sin \theta & \cos \theta \sin \theta & \cos \theta \cos \theta
\end{array}\right]
$$

where $\theta$ is the angle of misalignment, assuming all thrusters are misaligned by the same degree about each axis. The reader should note here that this research does not consider non-orthogonal misalignments 


\subsubsection{Thruster Bias}

A bias may also exist in the thrusters, where the control effort is not what is expected (usually less). This error is modeled by passing the control effort through a gain, $T B$, such that

$$
T B=1-\text { bias }
$$

where bias is the percentage of thrust lost.

\subsection{Gravitational Perturbations}

The following perturbations are a result of gravitational forces due to objects other than those specified in the CR3BP.

\subsubsection{Self Gravity}

The proximity of the leader and occulter spacecraft, themselves, cause gravitational effect on one another. This is modeled through the following two-body gravitational equation:

$$
\ddot{r}_{o l}=\frac{G\left(m_{l}+m_{o}\right)}{\left\|r_{o l}\right\|^{3}} \vec{r}_{o l} .
$$

where

$$
r_{o l}=r_{o}-r_{l}
$$

and $l$ and $o$ subscripts represent the leader and the occulter, respectively, $r$ is the distance from $\mathrm{L}_{2}, \mathrm{G}$ is the gravitational constant and $m$ is the corresponding spacecraft. 


\subsubsection{Gravitational Effects of Jupiter}

The gravitational force of Jupiter is considered since it is of significant mass located somewhat near Earth and has the largest gravitational force (aside from the sun) in our Terra System. This is also modeled through the two-body gravitational equation

$$
\ddot{r}_{J o}=\frac{G M_{J}}{\left\|r_{J o}\right\|^{3}} r_{J o}
$$

where

$$
r_{J o}=R_{J}-r_{o}
$$

$R_{J}$ is the distance from $\mathrm{L}_{2}$ to Jupiter, which remains constant and $M_{J}$ is the mass of Jupiter. The mass of the occulter is neglected since $M_{J}>>m_{0}$.

\subsection{Other Perturbations}

\subsubsection{Solar Radiation Pressure}

Solar radiation pressure (SRP) is the force exerted on an object due to the Sun's radiant energy. SRP is defined as

$$
\mathbf{S R P}=-\frac{p_{S R} c_{R} A_{o}}{m_{o}}\left[\begin{array}{l}
1 \\
0 \\
0
\end{array}\right]
$$

where $p_{S R}$ is the force per unit area due to the radiation, $c_{R}$ is the coefficient of reflectivity of the occulter, and $A_{o}$ is the area of the occulter seen by the Sun. For simplicity and without loss of generality, the occulter is assumed to have equal surface areas in each of the axial directions. Therefore $A_{o}$ is constant. SRP is assumed to constantly exert a force 
in the x-direction, since the spacecraft is considered to remain in the Sun's equatorial plane with the same level of exposure to the Sun.

\subsubsection{Measurement Noise}

When state measurements are used as feedback to the controller, measurement noise is also present. The measurement noise is modeled with the addition of band-limited white noise to the actual state being fed back. The levels of the power spectral density (PSD) are $100 \mathrm{~m}$ for position and $0.1 \mathrm{~mm} / \mathrm{s}$ for velocity, which have a correlation time of a tenth of a day.

position PSD noise level : $100 \mathrm{~m}$

velocity PSD noise level: $0.1 \mathrm{~mm} / \mathrm{s}$

\subsubsection{Differing Initial Conditions}

For all cases, the occulter is initialized at the initial conditions, $I C$, such that

$$
I C=\left[\begin{array}{c}
x_{0}-5000 m \\
y_{0}-5000 m \\
z_{0}-5000 m \\
0 \\
0 \\
0
\end{array}\right]
$$

where subscript $o$ represents the ideal initial conditions. 


\section{CHAPTER 5}

\section{CONTROL TECHNIQUES}

\subsection{Investigated Control Techniques}

As stated earlier, the leader and the occulter follow a lissajous orbit. The linearized dynamics of the circular restricted three body problem for this given lissajous trajectory results in a stable orbit. When modeled as a nonlinear system, feedback control is necessary to maintain a stable orbit. The basic form of a closed-loop spacecraft control system follows the same format seen in Figure 5.1, where the error between the reference state (the desired orbit) and the actual state, known as tracking error, is used to update the controller, which produces the required control effort to drive the error to zero. Disturbances can be internal or external perturbation, while the measurement system causes measurement noise.

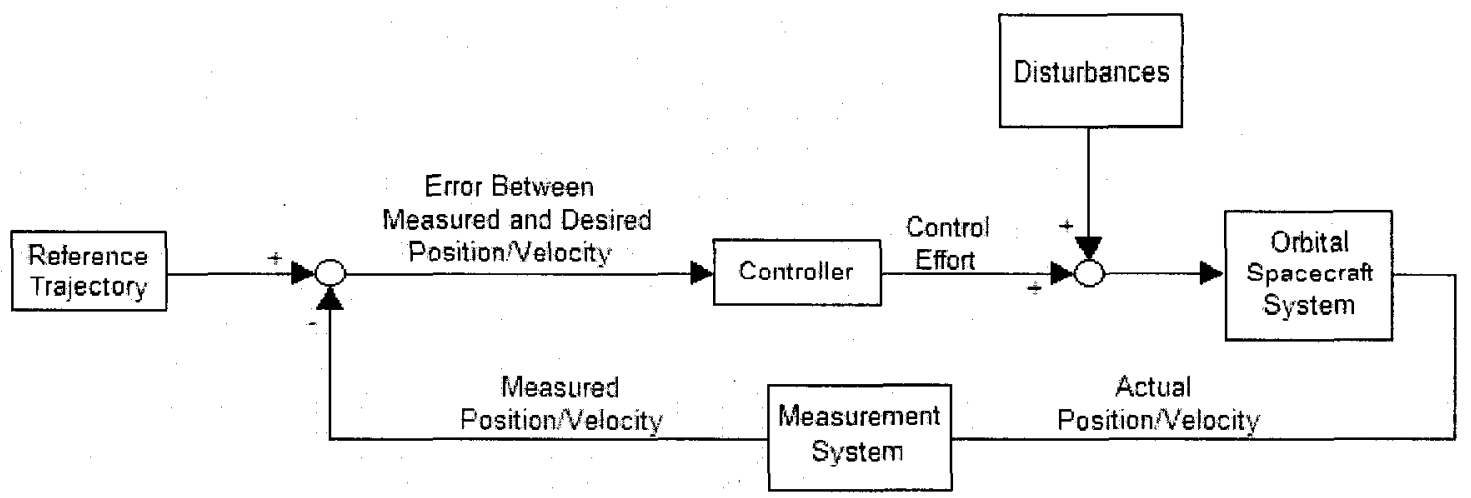

Figure 5.1 - Formation flying closed-loop control system 
Two extremes can occur when minimizing tracking error. If the tracking error converges to zero too quickly, a large control effort is needed. This effort can be decreased, although this will cause the tracking error to slowly dissipate. This property is directly related to the closed-loop poles of the system. Larger negative poles result in a faster decay of the error but require a large amount of control effort [3]. The mission in study requires the occulter tracking errors in the $\mathrm{x}-, \mathrm{y}$ - and $\mathrm{z}$-directions to remain within a ten meter tolerance from the desired position. The primary objective, however, is to minimize the control effort, while maintaining the given error tolerances.

This research focuses on four control techniques: three linear controllers and one nonlinear controller. The three linear controllers are PID control, Linear Quadratic Regulator (LQR) and $\mathrm{H}_{\infty}$. The nonlinear technique is a Sliding Mode Controller (SMC). The following sections discuss the properties of each of the controllers. The corresponding control law / dynamics are presented later in their respective chapters.

\subsubsection{Properties of PID Control}

PID control is comprised of three control gains: a proportional, an integral and a derivative. In general, each gain adjusts certain properties of the system dynamics. The proportional gain matrix has the greatest direct effect on the system response. Changing the gains of this matrix effects levels of oscillation, initial response time and control effort. The integral gain matrix is usually chosen to eliminate steady-state tracking error, while the derivative gain matrix reduces system overshoot or increase the rate of system response [4]. 


\subsubsection{Properties of Linear Quadratic Regulator}

The Linear Quadratic Regulator (LQR) is a form of optimal control which uses two matrices, $\mathbf{Q}$ and $\mathbf{R}$, which weight the relative importance of control effort and tracking error. The weighting matrices are used to find a solution to the steady state Riccati equation, the solution being the LQR gain matrix. Using the solution of the Riccati equation ensures. stable linear system closed loop poles. The gain and phase margins produced by $L Q R$ are very large, meaning that a system using an LQR controller will remain stable in the presence of disturbances. However, the LQR controller assumes a linear (or linearized nonlinear) system. Therefore, when implemented on the original nonlinear system, the same level of robustness as that of the linear (or linearized) system is not expected [4].

\subsubsection{Properties of H-Infinity}

$\mathrm{H}_{\infty}$ is another form of optimal linear control which also takes advantage of the Riccati equation. but which consists of different matrices than the $L Q R$. $\left(\mathrm{H}_{\infty}\right.$ is further discussed in Chapter 7.) The controller is based on a two-point boundary value problem. The lower boundary considers the problem of using minimal control effort to decrease tracking error. The upper boundary is the problem of decreasing tracking error in the presence of a calculated "maximum" disturbance. Since $\mathrm{H}_{\infty}$ provides a control law which minimizes control effort with a bounded disturbance, it can be considered a very robust type of control. But like LQR it is designed for a linear system and should not be assumed to maintain the same level of robustness when applied to a nonlinear system [5]. 


\subsubsection{Properties of Sliding Mode Control}

Sliding Mode Control (SMC) traditionally uses two components: an "equivalent" control term and a switching term. The equivalent control term generally may be freely chosen from any control technique but is often chosen to eliminate nonlinear dynamics and insert desired linear dynamics (i.e. feedforward with feedback control). The switching term ensures that the error trajectory is attracted to a sliding surface and remains on this surface. The sliding surface is a function of the state errors (explained in more detail in chapter 9). Once the error trajectory is on the sliding surface, SMC ensures that it remains on the surface, forces the error trajectory to the origin. For the general case, the equivalent control term contributes the greater effort, during the reaching phase, while the switching term ensures robustness against disturbances and perturbations while the error trajectory is on the sliding surface (the sliding phase).

\subsection{Other Control Techniques}

The following control techniques were considered in this research but were not included in the comparative study.

\subsubsection{Feedback Linearization}

Feedback linearization is a form of nonlinear control. The main principal behind the technique is to "feed back" a portion of the plant to eliminate nonlinear dynamics. Thus, the system can be analyzed as a linear dynamic system, producing the ability to 
incorporate any appropriate linear control technique. Feedback linearization, however, requires perfect knowledge of the system a priori, which is not always available in a spacecraft design or for the space environment. Without this a priori information, the controller is usually susceptible to parameter uncertainty and disturbances [3].

\subsubsection{Adaptive Control}

Adaptive control is another form of nonlinear control. Unlike feedback linearization, adaptive control is shown to be robust against parametric uncertainties. The technique uses the error between the actual plant and the ideal plant to "adapt" its control parameters to force the error trajectory to the origin. In this application however, the plant is assumed to be known exactly. The source of perturbation in the system originate primarily from external sources. Adaptive control is not as effective for modeling uncertainties and disturbances/perturbations that can not be characterized as a parametric uncertainty [3].

\subsubsection{Gain Scheduling}

Gain scheduling applies a linear control law to linearized regions of a nonlinear system. This is accomplished by linearizing the dynamic system about operating points and by designing appropriate controllers for each region to ensure stability. Between operating points, the gains of the controller are "scheduled" or interpolated. The biggest hindrance to this method is the large computational requirement. Also, in a highly nonlinear system, the operating points about which the system should be linearized, would need to be within close proximity to each other to prevent system instability, thus resulting in a 
greater required number of operating points and increased computational requirements [3]. 


\section{CHAPTER 6}

\section{PID CONTROL}

\subsection{Fundamental Equations}

PID is a linear controller which creates a control effort based on a gains that are proportional to the integral and derivative of the tracking error, as well as being directly proportional to the tracking error. Thus the control law becomes

$$
\mathbf{u}(t)=\mathbf{K}_{P} \mathbf{e}(t)+\mathbf{K}_{I} \int_{t_{0}}^{t} \mathbf{e}(\tau) d \tau+\mathbf{K}_{D} \frac{d \mathbf{e}}{d t}
$$

or in the Laplace domain

$$
\mathbf{U}(s)=\left[\mathbf{K}_{P}+\frac{\mathbf{K}_{I}}{s}+\mathbf{K}_{D} s\right] \mathbf{E}(s)
$$

where $\mathbf{K}_{P} \in \mathbb{R}^{3 \times 6}, \mathbf{K}_{I} \in \mathbb{R}^{3 \times 6}$ and $\mathbf{K}_{D} \in \mathbb{R}^{3 \times 6}$ are the proportional, integral and derivative gain matrices, respectively, for the dynamics of a three-dimensional orbital spacecraft system. When applied to the equations of motion of the CR3BP, the gain matrices follow

$$
\mathbf{K}=[\operatorname{diag}(\mathbf{p}), \operatorname{diag}(\mathbf{q})]>0
$$

where $\mathbf{p}$ and $\mathbf{q}$ are arrays of equal size and composed of elements which act on position and velocity errors, respectively. The gains are formatted in this fashion to create a control effort based on related states (e.g., $x$ and $\dot{x}$ ). The use of these three matrices 
allows the dynamic response of the system to be more intuitively manipulated by the user, as described in section 5.2.1.

Figure 6.1 shows the updated closed-loop diagram of Figure 5.1.

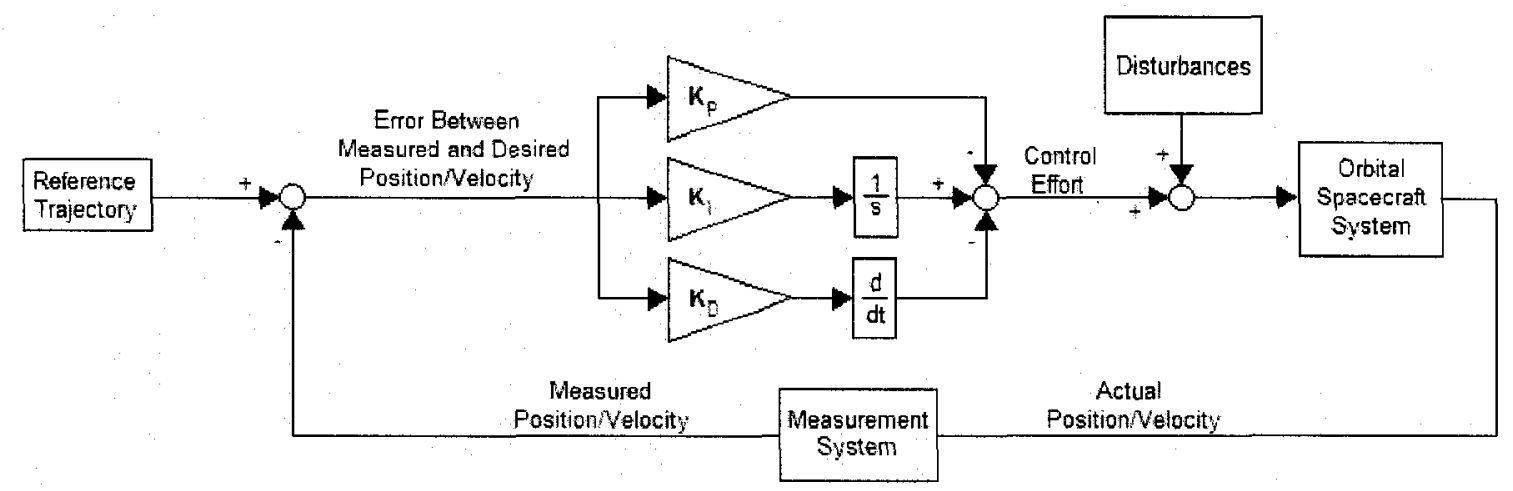

Figure 6.1 - Simplified PID block diagram

\subsection{Gain Numbers}

The gain selection process for the PID controller begins with selecting gains for the linearized CR3BP system through intuitive trial and error deduction. Combinations of P-, I- and D-control are investigated until a desired transient response is obtained. These gains are then applied to the nonlinear CR3BP system which are then tuned appropriately until the following gains are obtained:

$$
\mathbf{K}_{P}=\left[\begin{array}{cccccc}
4 \cdot 10^{-4} & 0 & 0 & 2 \cdot 10^{-5} & 0 & 0 \\
0 & 9 \cdot 10^{-6} & 0 & 0 & 7 \cdot 10^{-13} & 0 \\
0 & 0 & 10^{-5} & 0 & 0 & 4 \cdot 10^{-11}
\end{array}\right]
$$




$$
\begin{gathered}
\mathbf{K}_{I}=\left[\begin{array}{cccccc}
10^{-12} & 0 & 0 & 3 \cdot 10^{-17} & 0 & 0 \\
0 & 6 \cdot 10^{-15} & 0 & 0 & 0 & 0 \\
0 & 0 & 10^{-17} & 0 & 0 & 0
\end{array}\right] \\
\mathbf{K}_{D}=\left[\begin{array}{cccccc}
10^{-4} & 0 & 0 & 0 & 0 & 0 \\
0 & 10^{-4} & 0 & 0 & 10^{-3} & 0 \\
0 & 0 & 10^{-4} & 0 & 0 & 0
\end{array}\right]
\end{gathered}
$$

These gains yield the results seen in the rest of the chapter.

\subsection{Unperturbed System Results}

Results pertaining to the PID controller applied to the nonlinear system without disturbances or perturbations are shown in Figures 6.2 through 6.4.
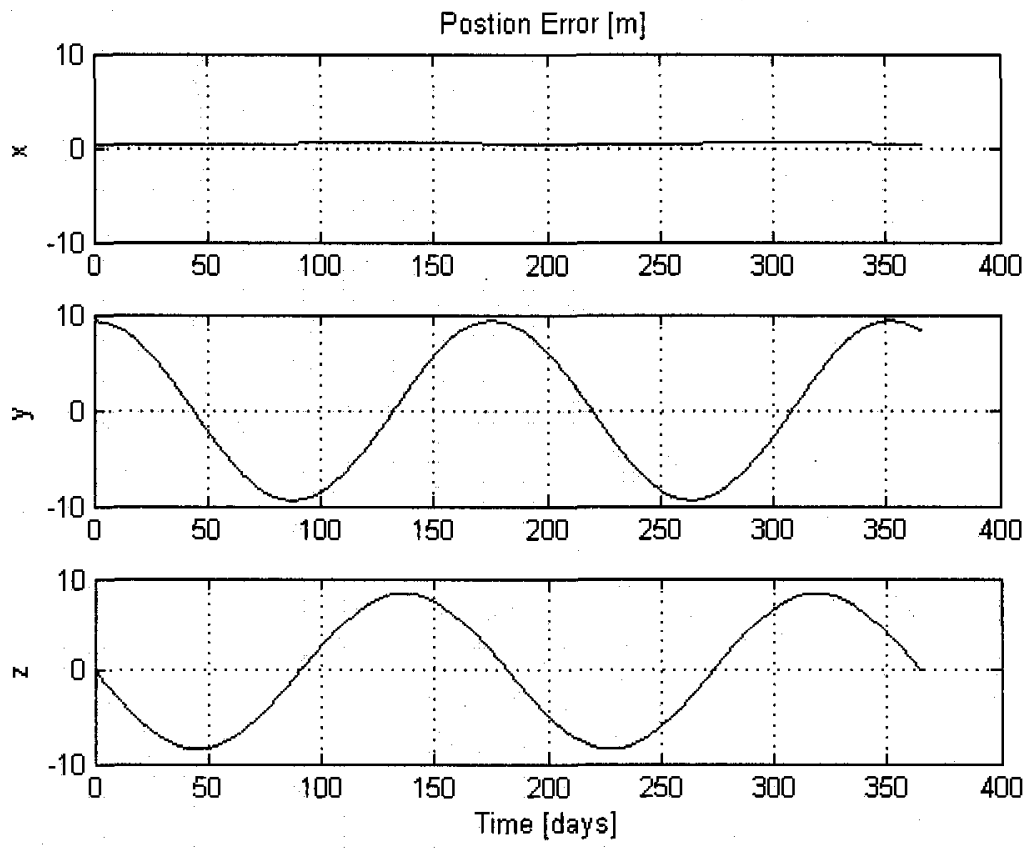

Figure 6.2 - PID position errors for 1 year (unperturbed) 

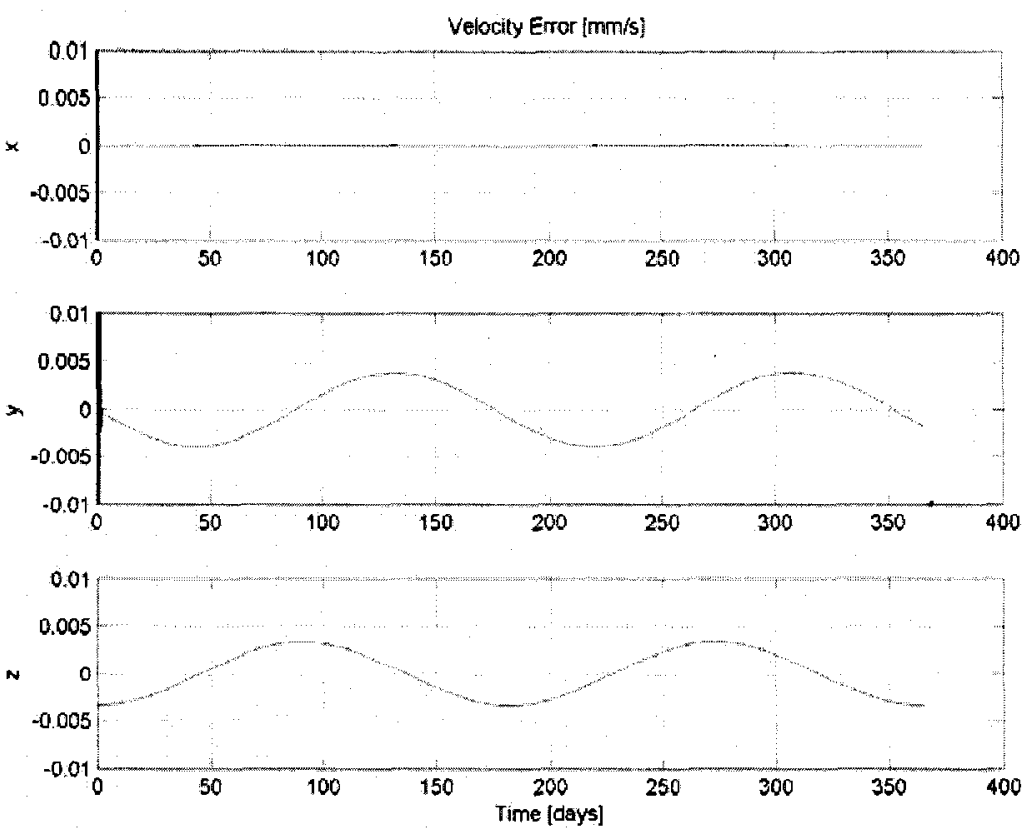

Figure 6.3 - PID velocity errors for 1 year (unperturbed)

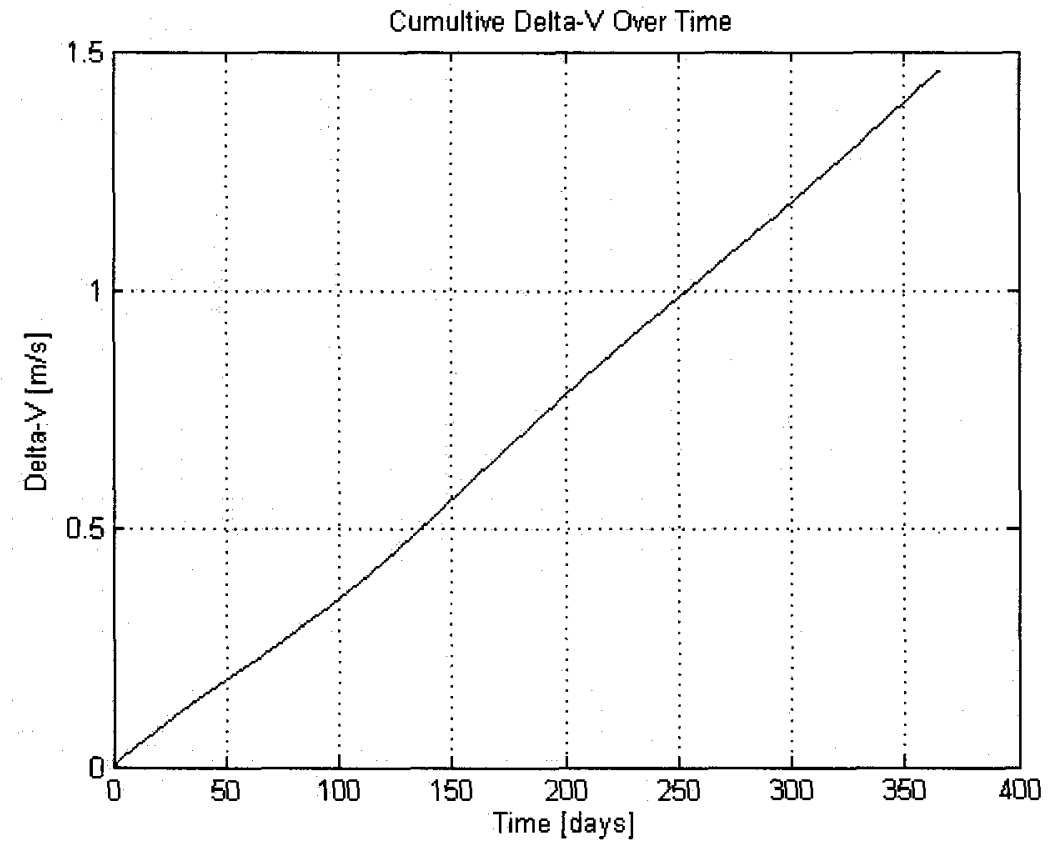

Figure 6.4 - PID $\Delta v$ profile for 1 year (unperturbed) 


\begin{tabular}{|c|c|c|c|c|}
\cline { 2 - 5 } \multicolumn{1}{c|}{} & \multicolumn{3}{c|}{ Maximum Erors } \\
\cline { 2 - 5 } \multicolumn{1}{c|}{} & $\mathbf{x}$ & $\mathbf{y}$ & $\mathbf{z}$ & \\
\hline position [m] & 0.55 & 9.3 & 8.4 & 1.46 \\
\hline velocity [pm]s] & 0.056 & 3.9 & 3.4 & 1.46 \\
\hline
\end{tabular}

Table 6.1 - PID results for 1 year (unperturbed)

Table 6.1 lists the $\Delta v$ and maximum absolute value of errors for position and velocity once the orbit is stabilized. The controller performs well, maintaining errors within the allowable tolerances. The $\mathrm{x}$-position error remains very small but shows a small bias. The $y$ - and z-position errors are larger but oscillate within the ten meter allowance. All cases develop large initial velocity errors. This is due to the extreme sensitivity of a natural orbit to its initial velocity conditions. As mentioned in Chapter 3, the reference lissajous orbit is propagated with the linear system to numerically obtain an orbit which is natural, however the propagation begins with the calculated initial velocities which are not natural. Figure 6.3 shows that the system quickly recovers from the unnatural initial velocities. This is an acceptable error, however, since the mission does not require velocity errors to be minimized. Figure 6.4 shows the $\Delta v$ profile over one year. It can be seen that the $\Delta v$ profile is nearly linear, meaning that fuel is used at a near constant rate over the one year orbit. Most importantly, though, $\Delta \mathrm{v}$ is kept to a minimal value while maintaining the trajectory. 


\subsection{Thruster Misalignment Results}

Results pertaining to the PID controller applied to the nonlinear system with thruster misalignments of a tenth of a degree are shown in Figures 6.5 through 6.7. Numerical results are listed in Table 6.2 .
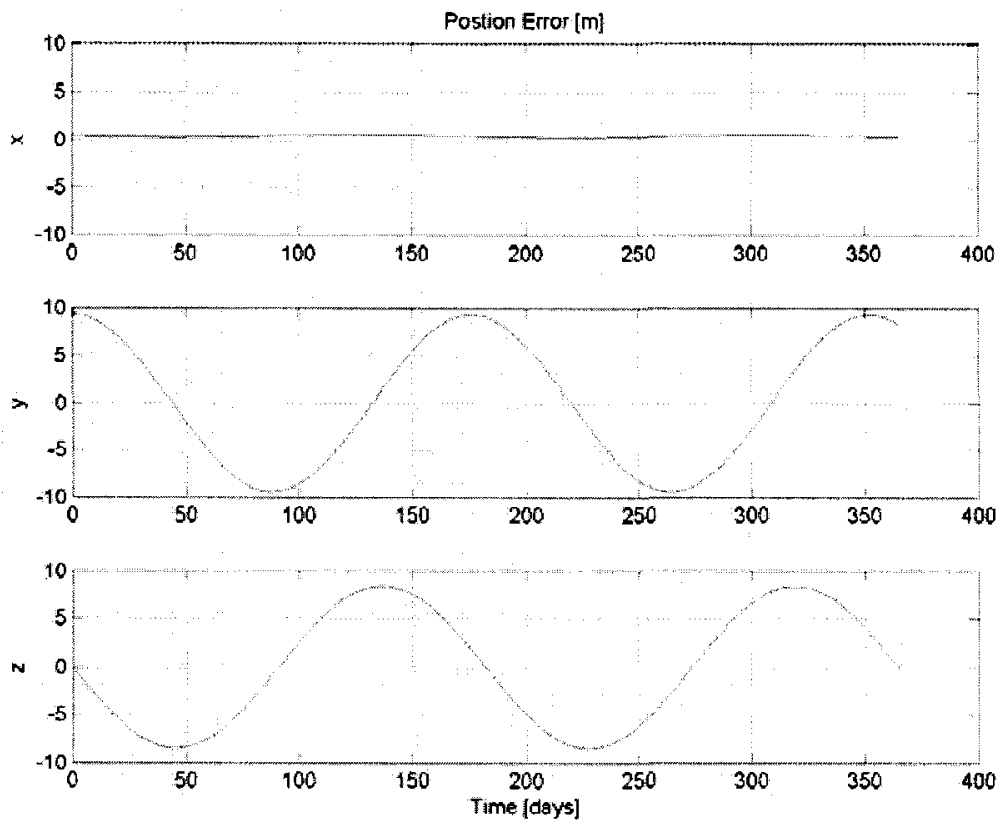

Figure 6.5 - PID position errors for 1 year (thruster misalignments) 

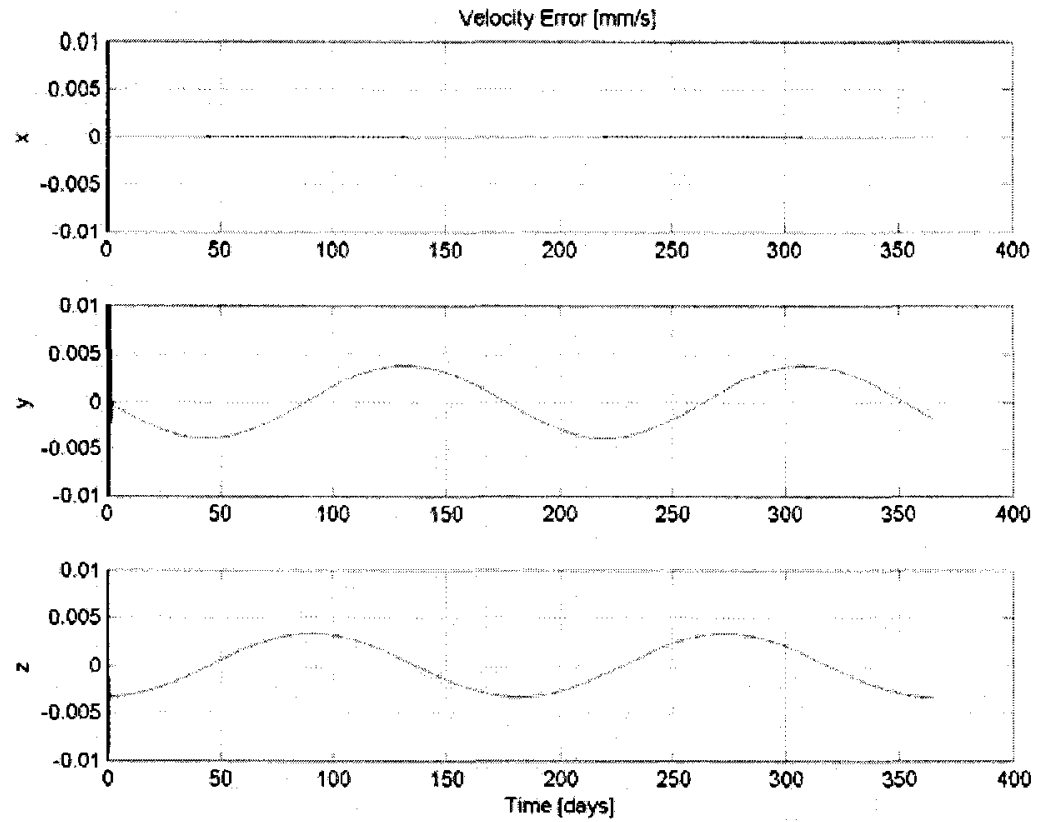

Figure 6.6 - PID velocity errors for 1 year (thruster misalignments)

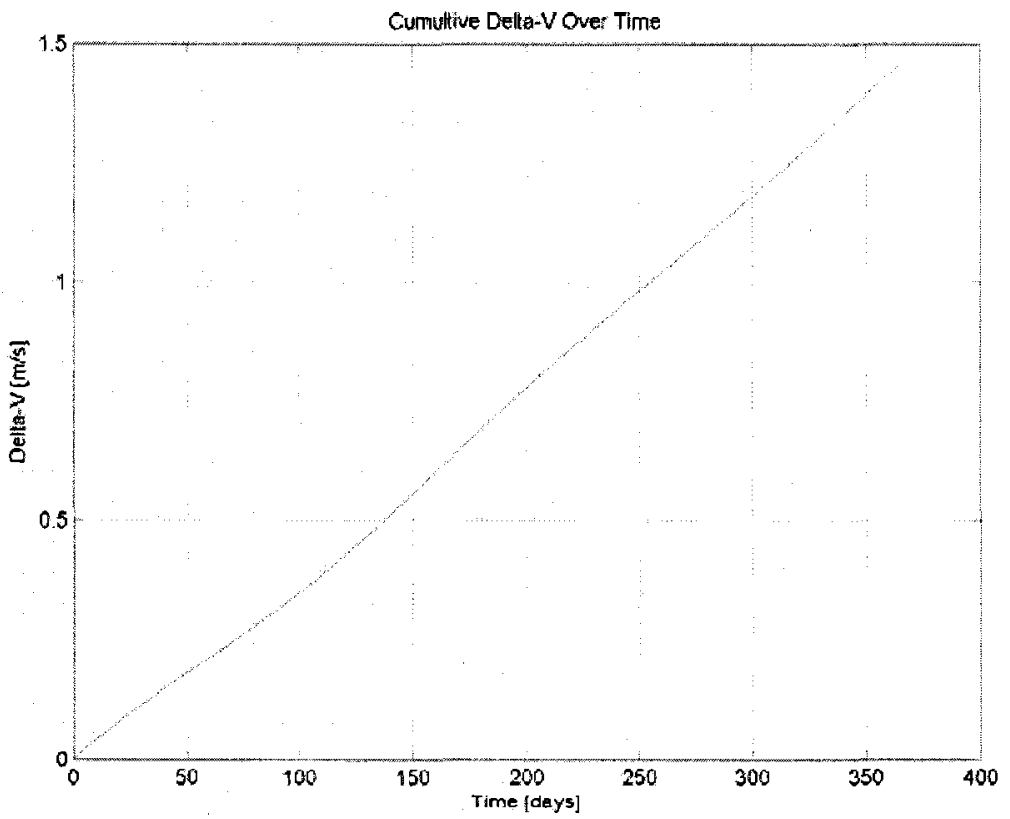

Figure 6.7 - PID $\Delta \mathrm{v}$ profile for 1 year (thruster misalignments) 


\begin{tabular}{|c|c|c|c|c|}
\hline & \multicolumn{3}{|c|}{ Maximum Errors } & \multirow{2}{*}{ 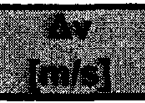 } \\
\hline & $x^{2}$ & $y$ & $z$ & \\
\hline position $[\mathrm{m}$ & 0.55 & 9.4 & 8.4 & \multirow{2}{*}{1.46} \\
\hline velocity [pm/s] & 0.056 & 3.9 & 3.4 & \\
\hline
\end{tabular}

Table 6.2 - PID results for 1 year (thruster misalignments)

The controller performs well, maintaining the errors close to those of the unperturbed system and maintaining the minimal $\Delta \mathrm{v}$. The PID controller, therefore, is shown to provide stability against the expected level of given thruster misalignments.

\subsection{Thruster Bias Results}

Results pertaining to the PID controller applied to the nonlinear system with thruster biases of $-5 \%$ are shown in Figures 6.8 through 6.10. Numerical results are listed in Table 6.3. 

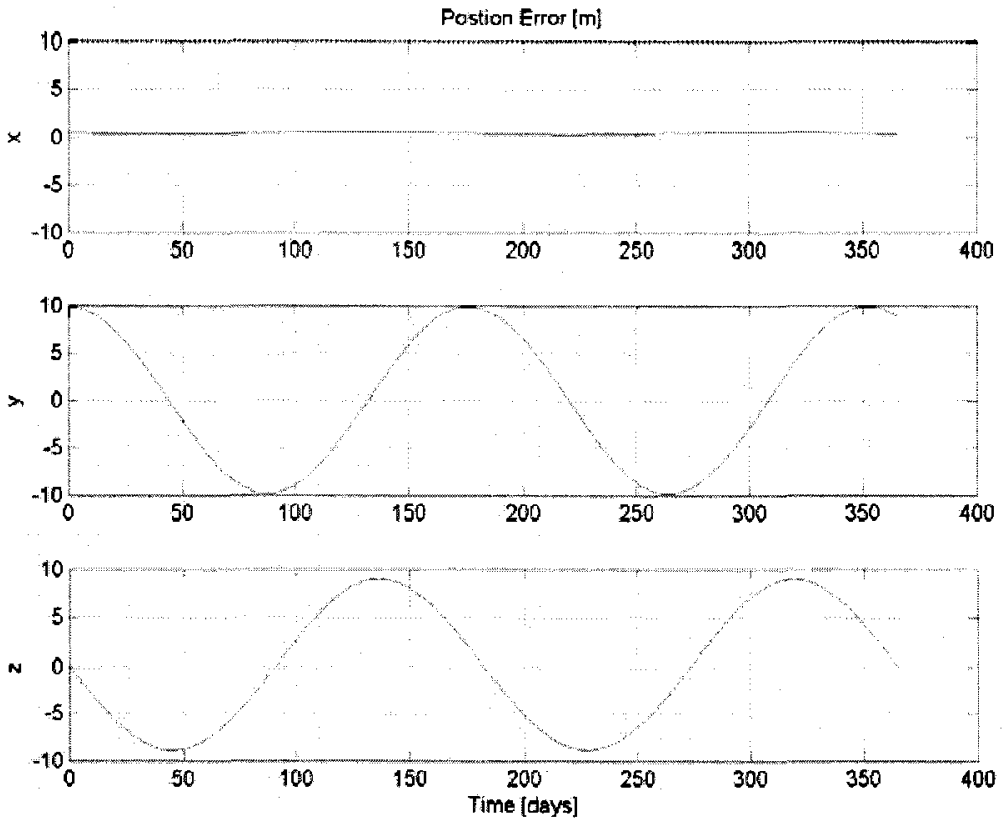

Figure 6.8 - PID position errors for 1 year (thruster bias)
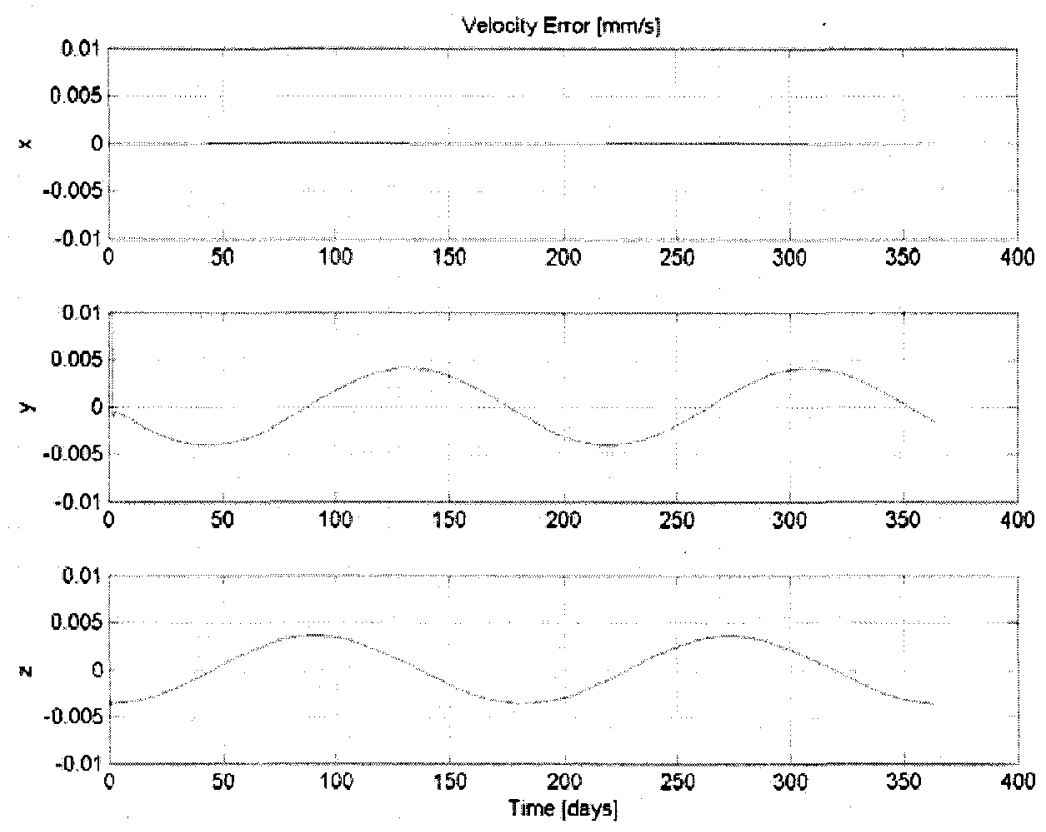

Figure 6.9 - PID velocity errors for 1 year (thruster bias) 


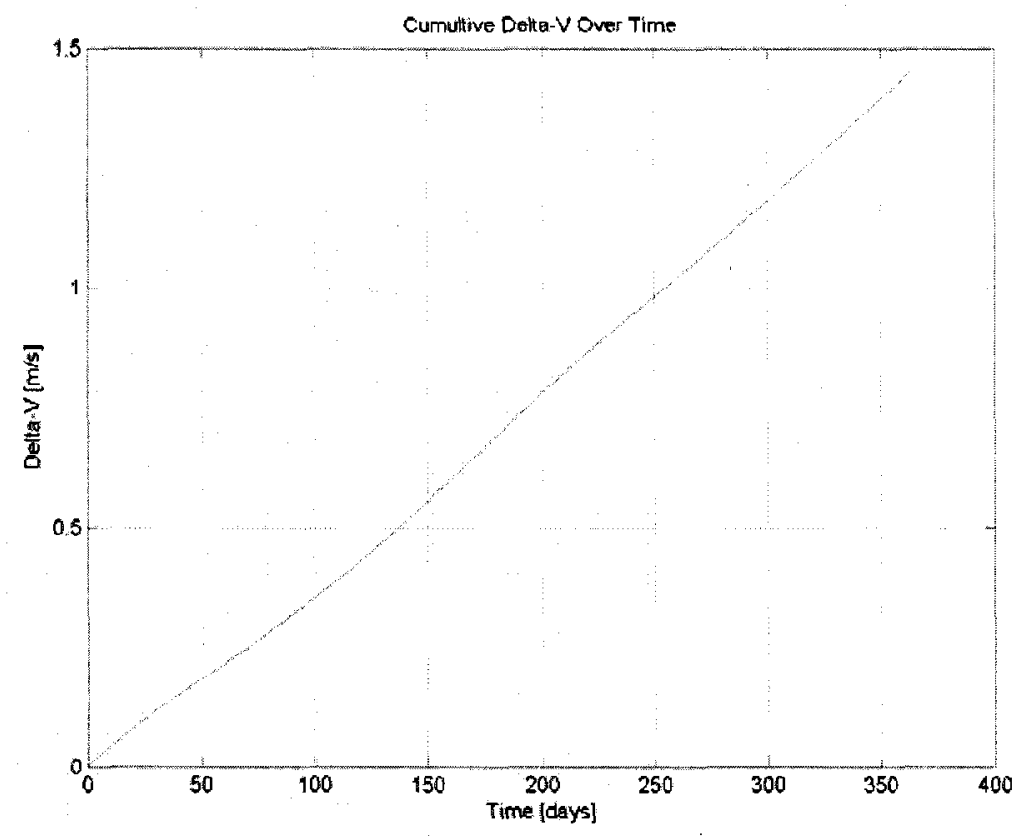

Figure 6.10 - PID $\Delta \mathrm{v}$ profile for 1 year (thruster bias)

\begin{tabular}{|c|c|c|c|c|}
\hline & \multicolumn{3}{|c|}{ Maximum Errors } & \multirow{2}{*}{ 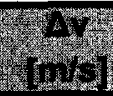 } \\
\hline & $\mathbf{x}$ & $y$ & $z$ & \\
\hline position [m] & 0.58 & 9.8 & 8.8 & \multirow{2}{*}{1.46} \\
\hline veloctty [pm/s] & 0.059 & 4.1 & 3.5 & \\
\hline
\end{tabular}

Table 6.3 - PID results for 1 year (thruster bias)

Figures 6.9 through 6.11 show that the PID maintains errors within the tolerance while exerting a minimal $\Delta \mathrm{v}$, showing that PID control is able to withstand the expected level of given thruster bias. 


\subsection{Self-Gravity Results}

Results pertaining to the PID controller applied to the nonlinear system with self-gravity from the leader satellite are shown in Figures 6.11 through 6.13. Numerical results are listed in Table 6.4.
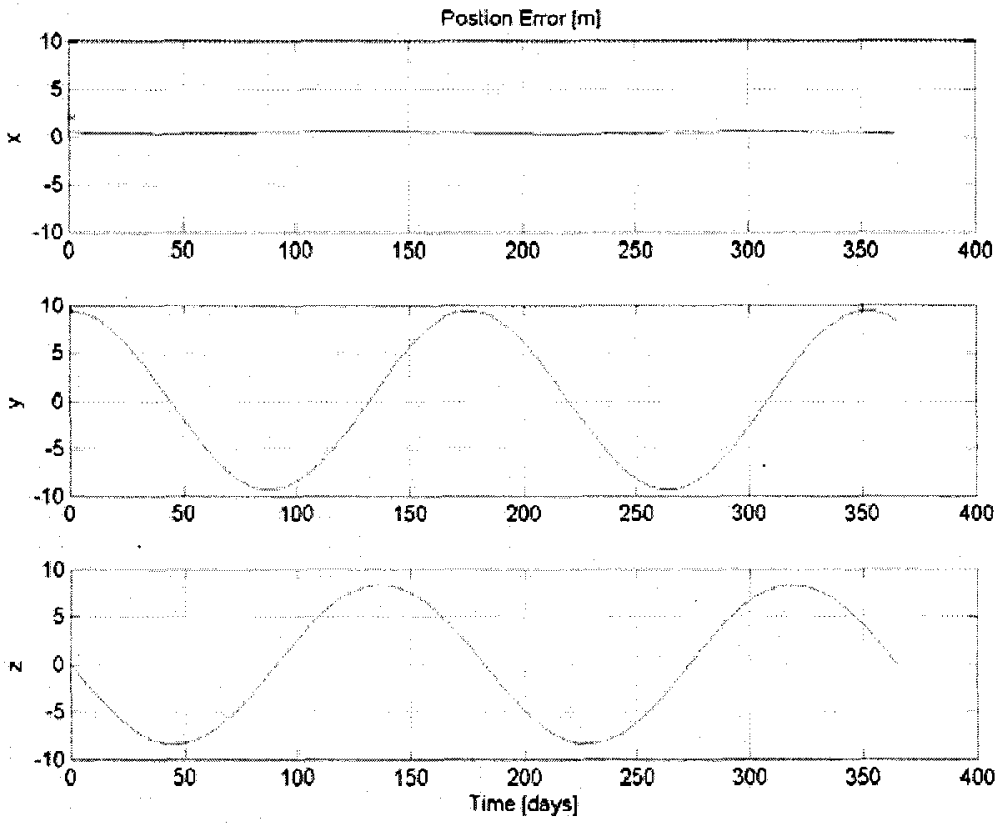

Figure 6.11 - PID position errors for 1 year (self-gravity) 

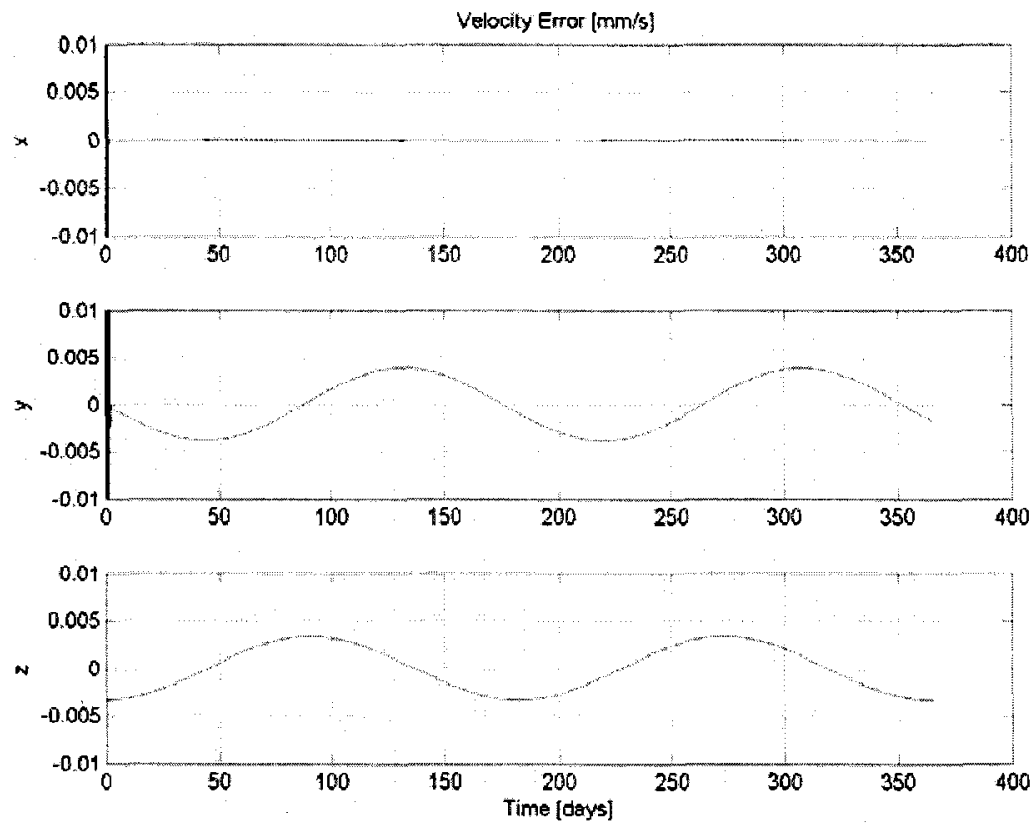

Figure 6.12 - PID velocity errors for 1 year (self-gravity)

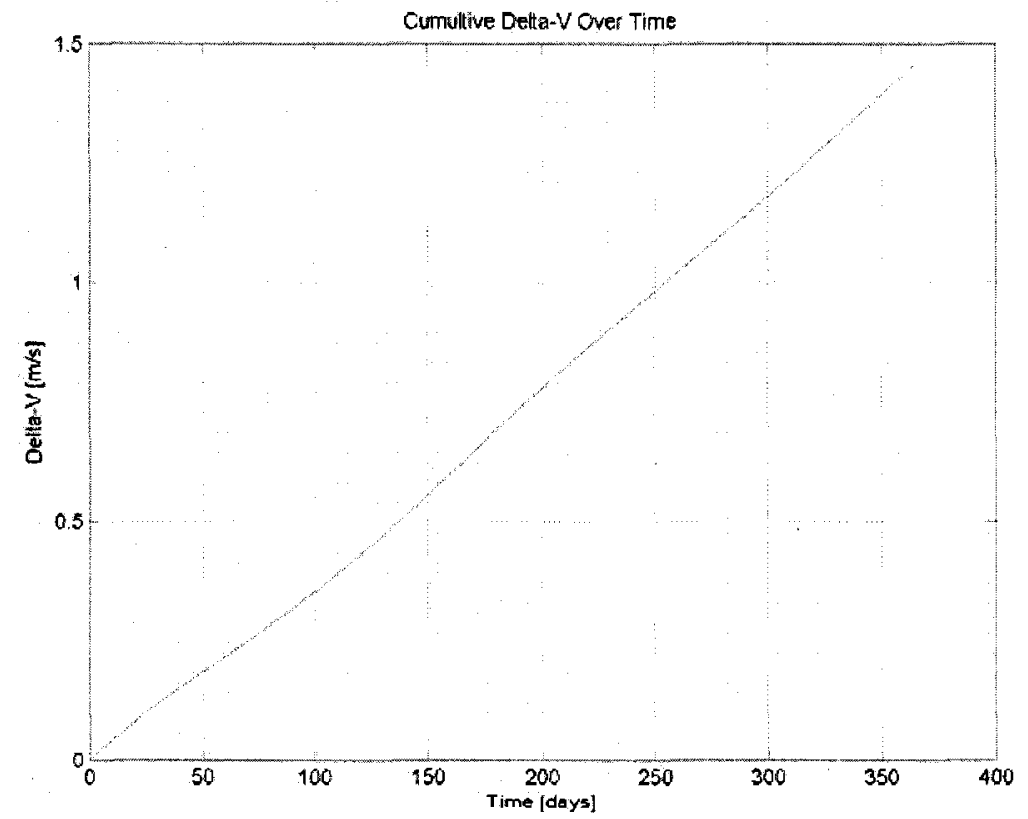

Figure 6.13 - PID $\Delta v$ profile for 1 year (self-gravity) 


\begin{tabular}{|c|c|c|c|c|}
\cline { 2 - 5 } \multicolumn{1}{c|}{} & \multicolumn{3}{c|}{ Maximum Errors } & \\
\cline { 2 - 5 } \multicolumn{1}{c|}{} & $\mathbf{X}$ & $\mathbf{Y}$ & $\mathbf{Z}$ & \\
\hline position [m] & 0.56 & 9.3 & 8.4 & 1.46 \\
\hline volocity [um/s] & 0.056 & 3.9 & 3.4 & \\
\hline
\end{tabular}

Table 6.4 - PID results for 1 year (self-gravity)

Once again, the errors and $\Delta \mathrm{v}$ are kept to a minimum and the PID controller is shown to not be affected by self-gravity from the leader.

\subsection{Jupiter Gravity Results}

The gravitational forces of Jupiter are implemented in the PID control system, and the results are shown in Figures 6.14 through 6.16. Numerical results are listed in Table 6.5.
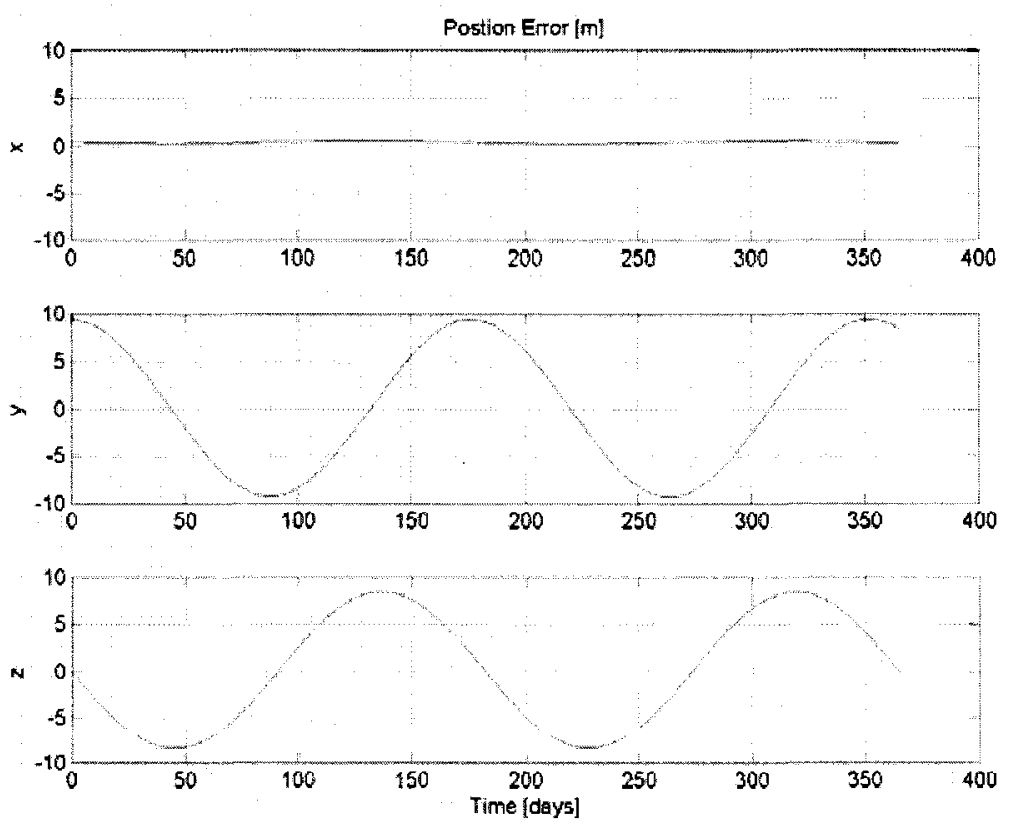

Figure 6.14 - PID position errors for 1 year (Jupiter) 

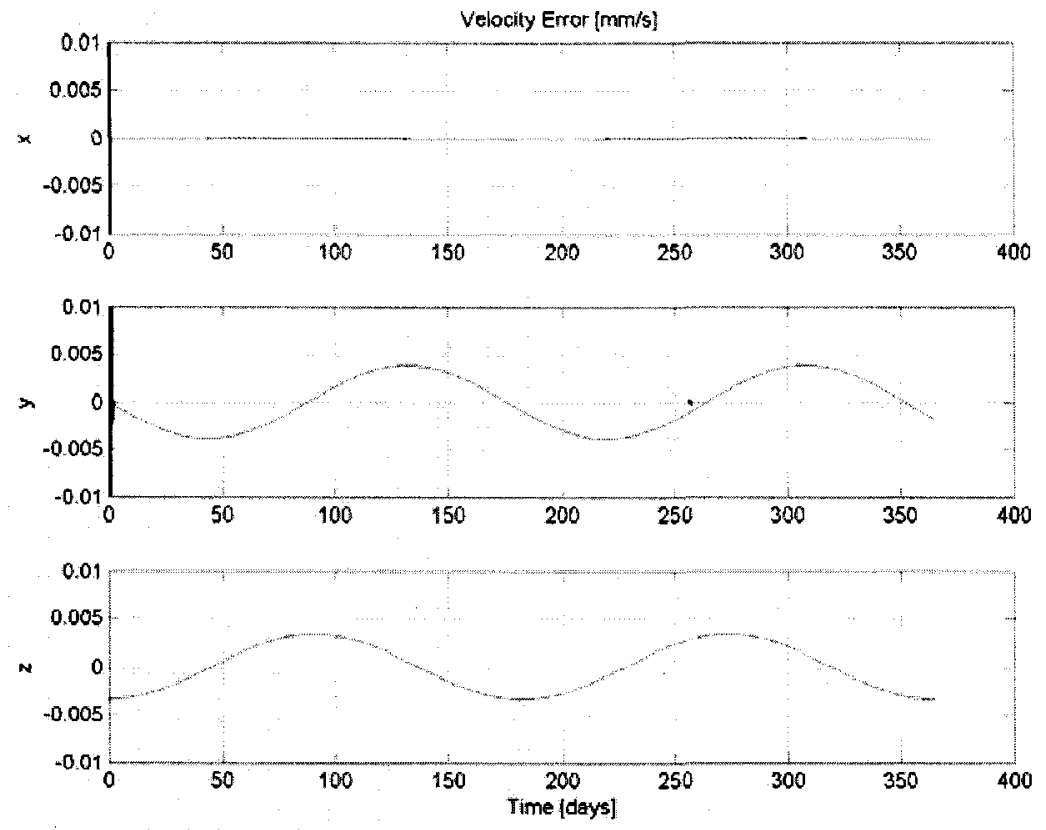

Figure 6.15 - PID velocity errors for 1 year (Jupiter)

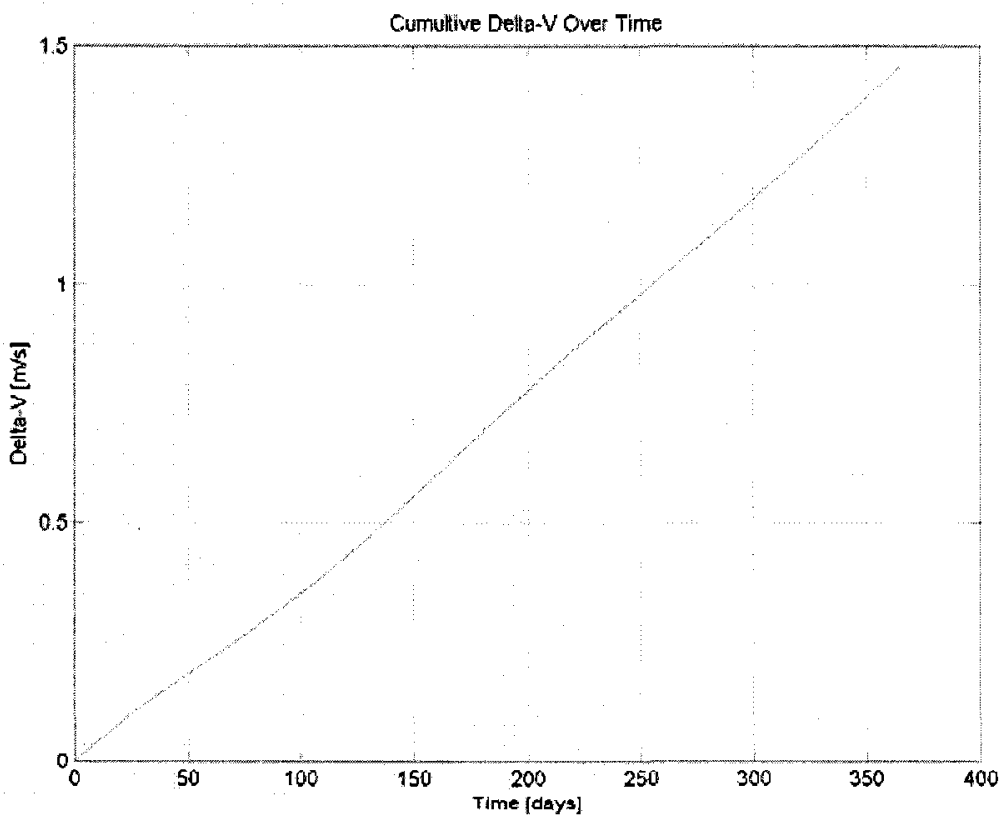

Figure 6.16 - PID $\Delta v$ profile for 1 year (Jupiter) 


\begin{tabular}{|c|c|c|c|c|}
\hline & \multicolumn{3}{|c|}{ Maximum Errors } & \multirow{2}{*}{ Shy } \\
\hline & $x$ & $y$ & 2 & \\
\hline posi & 0.55 & 9.3 & 8.4 & \multirow{2}{*}{1.46} \\
\hline Velocity [ [m/s] & 0.056 & 3.9 & 3.4 & \\
\hline
\end{tabular}

Table 6.5 - PID results for 1 year (Jupiter)

The controller performs well, yielding errors equal to those of the unperturbed system and maintaining the minimal $\Delta \mathrm{v}$. The PID controller is shown to pass mission requirements in the presence of gravitational forces due to Jupiter.

\subsection{Solar Radiation Pressure Results}

Results pertaining to the PID controller applied to the nonlinear system with the presence of solar radiation pressure are shown in Figures 6.17 through 6.19. Numerical results are listed in Table 6.6. 

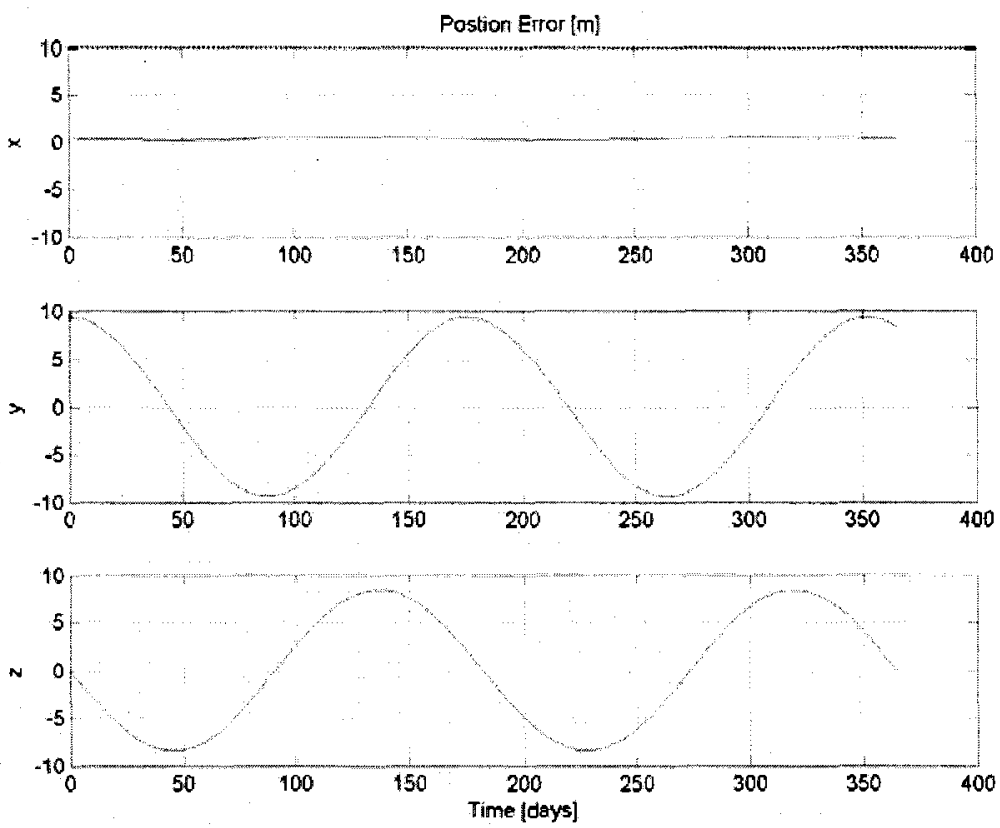

Figure 6.17 - PID position errors for 1 year (solar radiation pressure)
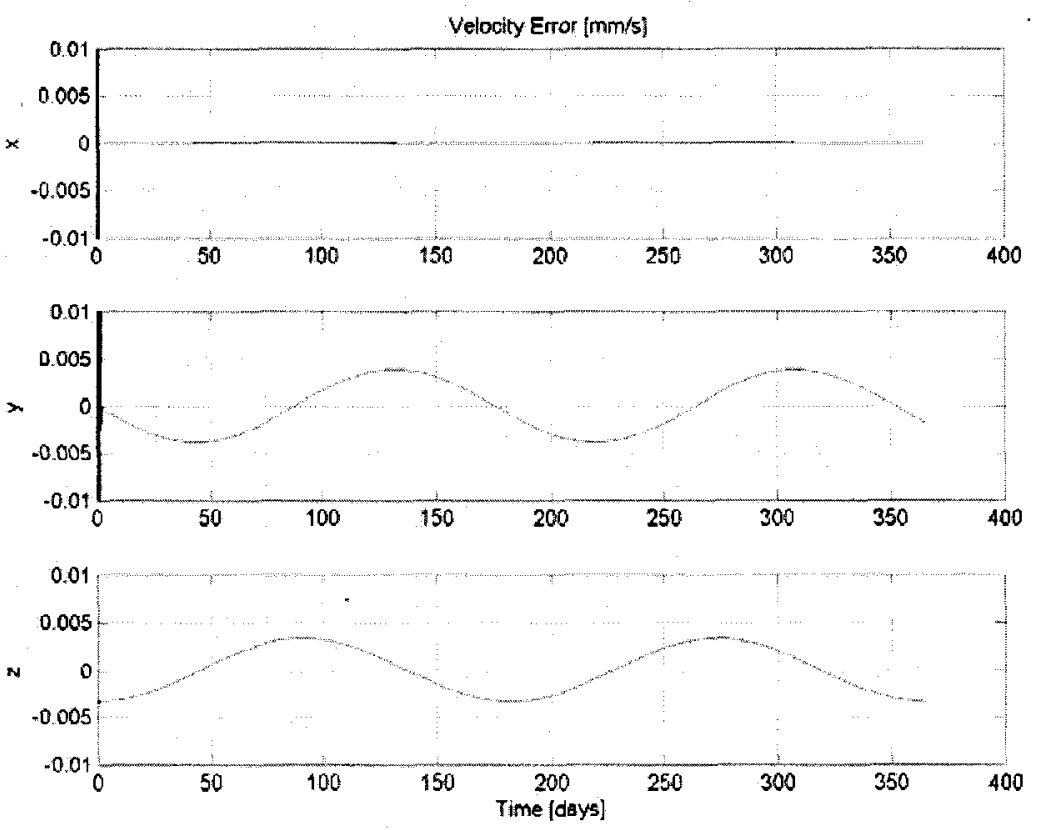

Figure 6.18 - PID velocity errors for 1 year (solar radiation pressure) 


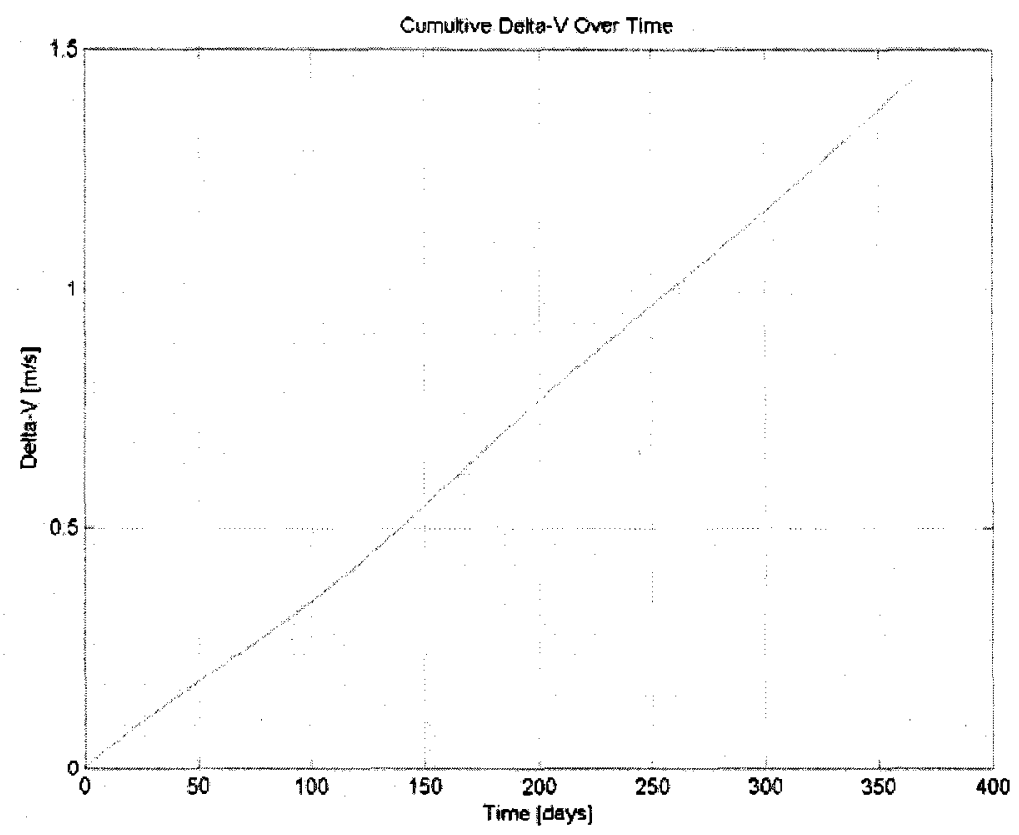

Figure 6.19 - PID $\Delta v$ profile for 1 year (solar radiation pressure)

\begin{tabular}{|c|c|c|c|c|}
\hline & \multicolumn{3}{|c|}{ Maximum Errors } & \multirow{2}{*}{ thet } \\
\hline & $x$ & $y$ & 2 & \\
\hline \multirow{2}{*}{$\begin{array}{l}\text { position }[\mathrm{m}] \\
\text { velocity }[\mu \mathrm{m} / \mathrm{s}]\end{array}$} & 0.55 & 9.3 & 8.4 & \multirow{2}{*}{1.43} \\
\hline & 0.056 & 3.9 & 3.4 & \\
\hline
\end{tabular}

Table 6.6 - PID results for 1 year (solar radiation pressure)

The controller is effective at maintaining errors within the tolerance while minimizing $\Delta v$. SRP is negligible against PID. 


\subsection{Measurement Noise Results}

Results pertaining to the PID controller applied to the nonlinear system with the presence of measurement noise are shown in Figures 6.20 through 6.22. Numerical results are listed in Table 6.7.
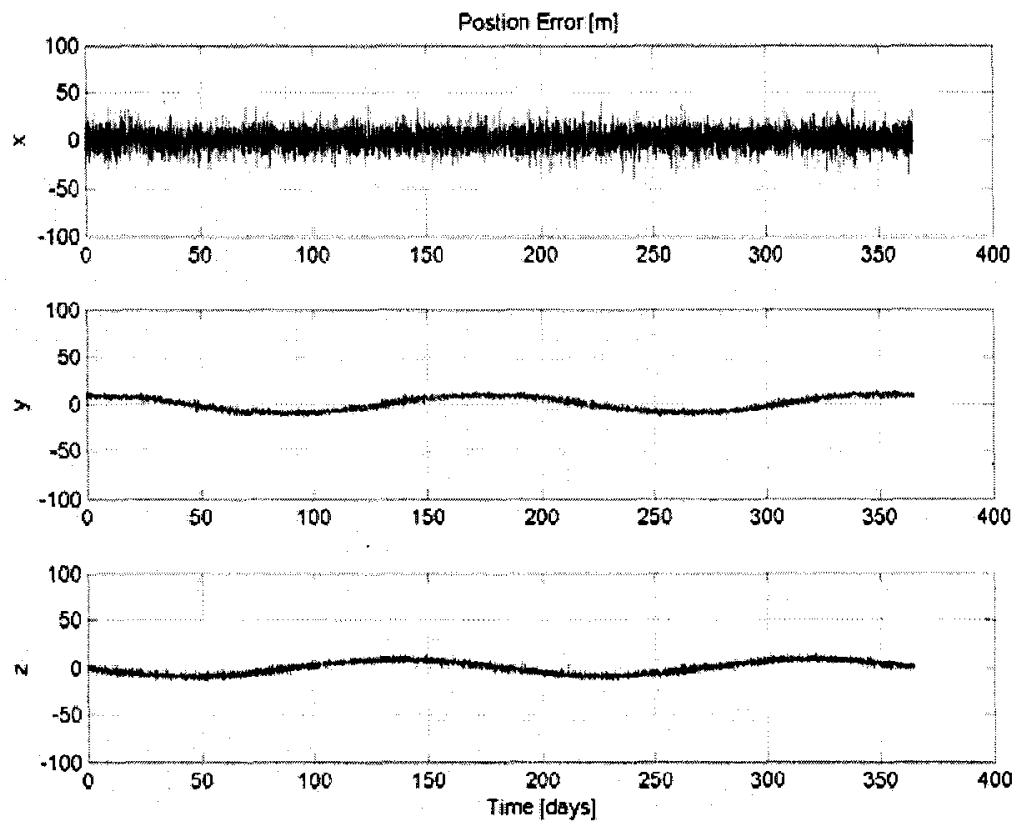

Figure 6.20 - PID position errors for 1 year (measurement noise) 

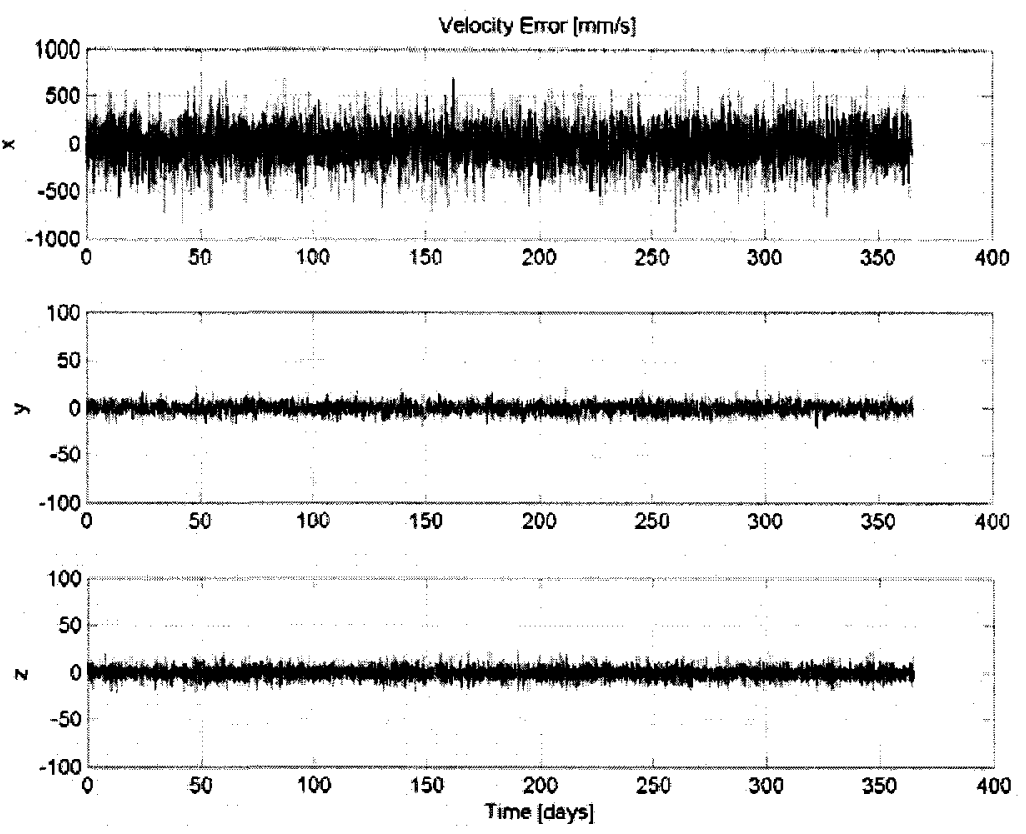

Figure 6.21 - PID velocity errors for 1 year (measurement noise)

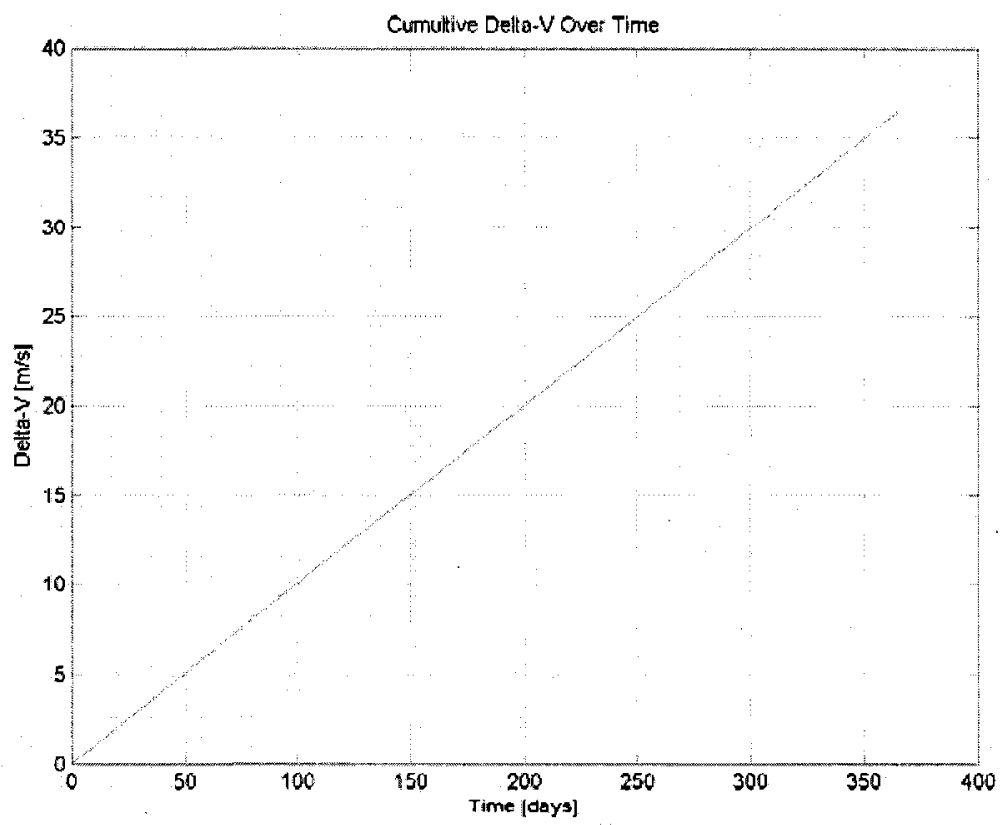

Figure 6.22 - PID $\Delta v$ profile for 1 year (measurement noise) 


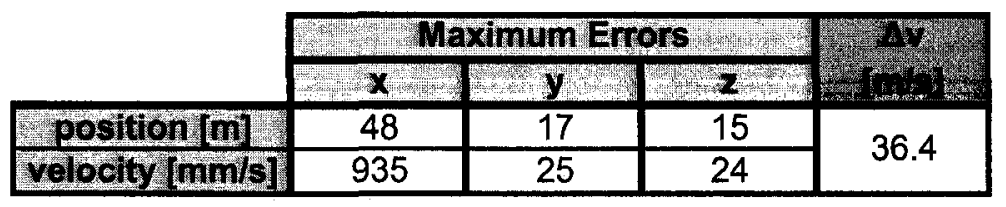

Table 6.7 - PID results for 1 year (measurement noise)

The performance of the PID controlled system degrades in the presence of measurement noise. The errors increase above acceptable mission requirements and $\Delta \mathrm{v}$ increases greatly as well. It is evident that the PID controller is not designed to perform against measurement noise. The derivative gain exists to decrease overshoot in the system, but hinders the system in the presence of noise for this mission scenario. When a rapidly changing, high-level white-noise is differentiated, the result is an even nosier signal, which, in turn, produces undesirable position and velocity adjustments. Therefore, PID control is not satisfactory in the presence of a reasonable level of given measurement noise.

\subsection{Differing Initial Conditions Results}

Results pertaining to the PID controller applied to the nonlinear system with incorrect initial conditions are shown in Figures 6.23 through 6.25. Numerical results are listed in Table 6.8. 

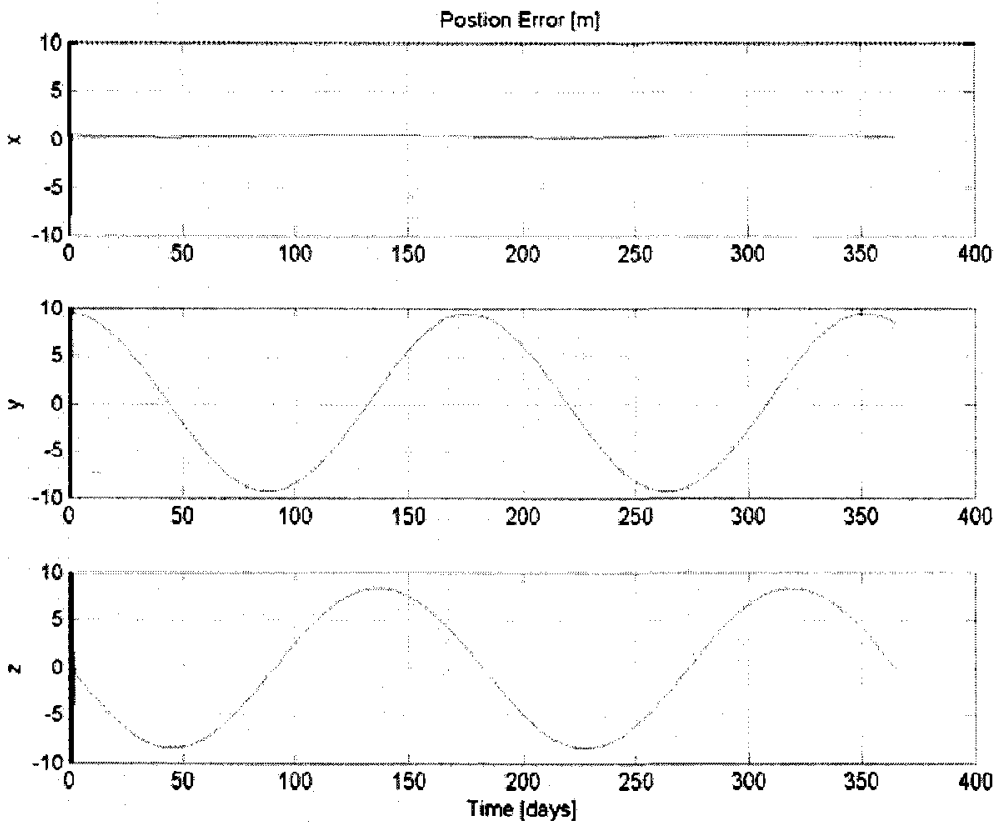

Figure 6.23 - PID position errors for 1 year (differing initial conditions)
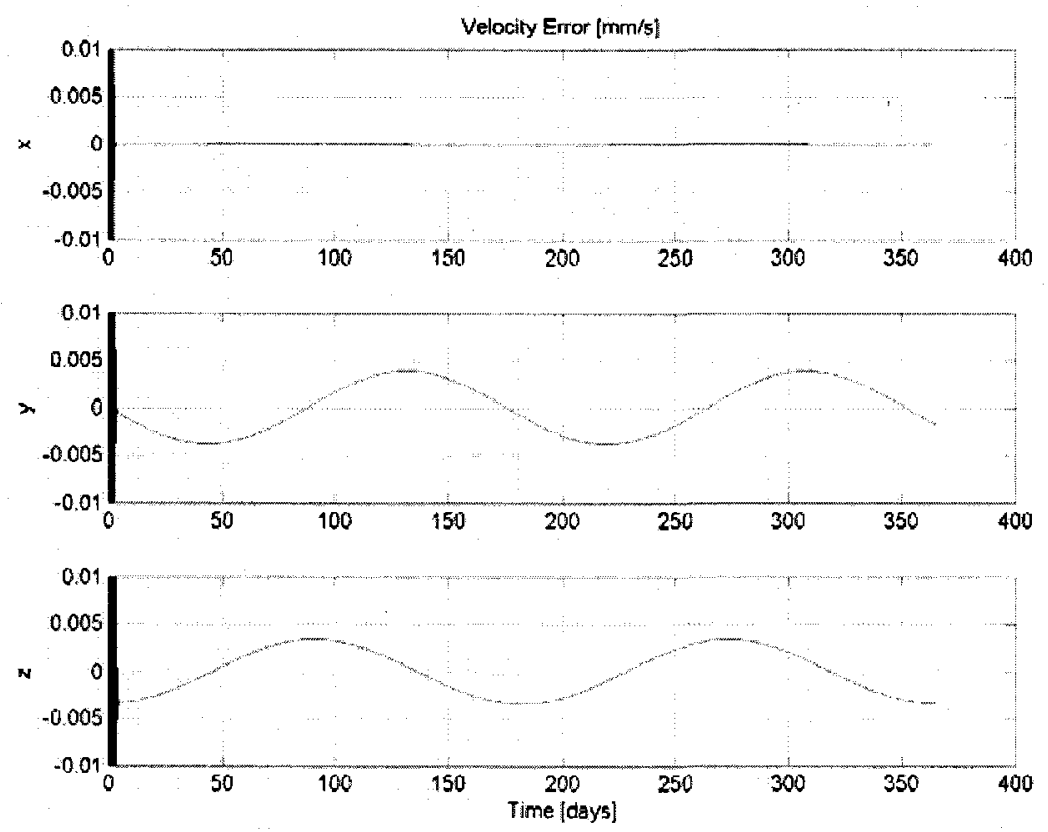

Figure 6.24 - PID velocity errors for 1 year (differing initial conditions) 


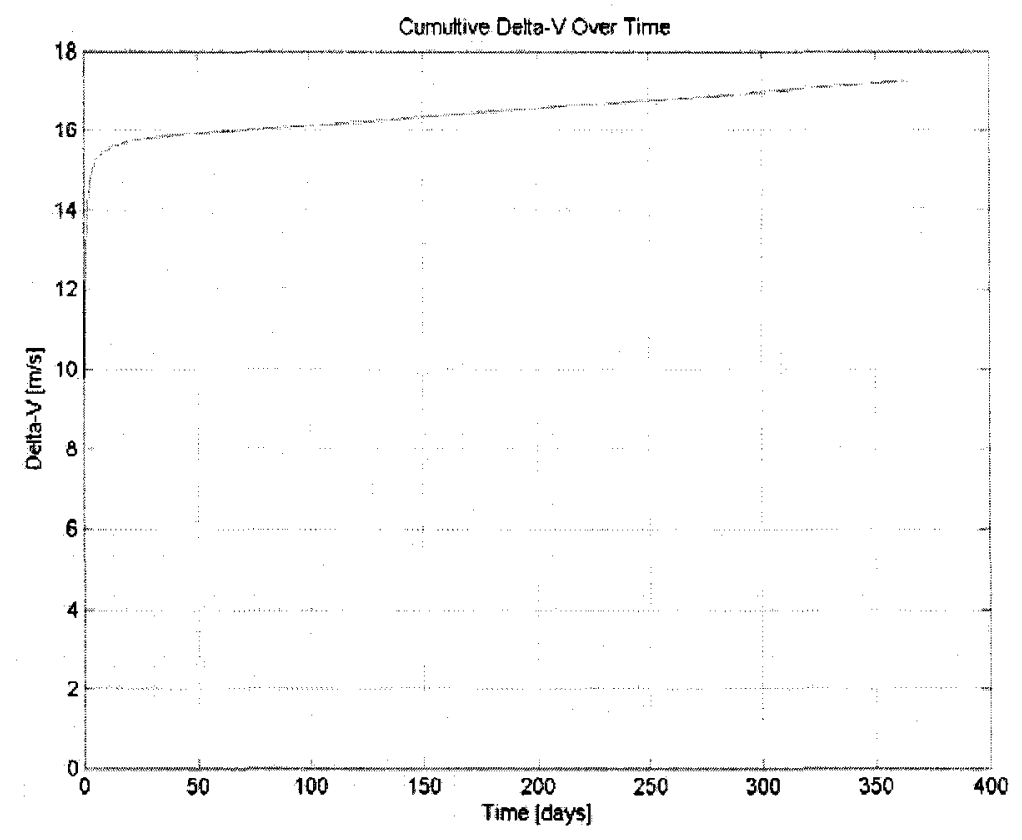

Figure 6.25 - PID $\Delta v$ profile for 1 year (differing initial conditions)

\begin{tabular}{|c|c|c|c|c|}
\hline & \multicolumn{3}{|c|}{ Maximum Errors } & \multirow{2}{*}{ 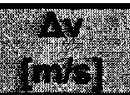 } \\
\hline & $x$ & $=y$ & 2 & \\
\hline position [m] & 0.56 & 9.3 & 8.4 & \multirow{2}{*}{17.2} \\
\hline velocity $[p m / s]$ & 0.056 & $\overline{3.9}$ & 3.4 & \\
\hline
\end{tabular}

Table 6.8 - PID results for 1 year (differing initial conditions)

The PID controlled system does well in maintaining errors within the mission allowance but does not maintain a minimum $\Delta \mathrm{v}$. This comes from the initial design of the controller, which is designed to allow for a quick recovery as errors begin to increase. With a large initial offset, the transient response exhibits highly oscillatory behavior, expending a large amount fuel in the early stage of the mission. This can be seen in Figure 6.25 with the large $\Delta v$ expenditure in the first couple days, which then decreases once the error tolerances have been reached. Therefore, PID control is not robust against differing initial conditions. 


\subsection{Worst Case Scenario Results}

Results pertaining to the PID controller applied to the nonlinear system with the presence of all modeled disturbances and perturbations are shown in Figures 6.26 through 6.28. Numerical results are listed in Table 6.9.
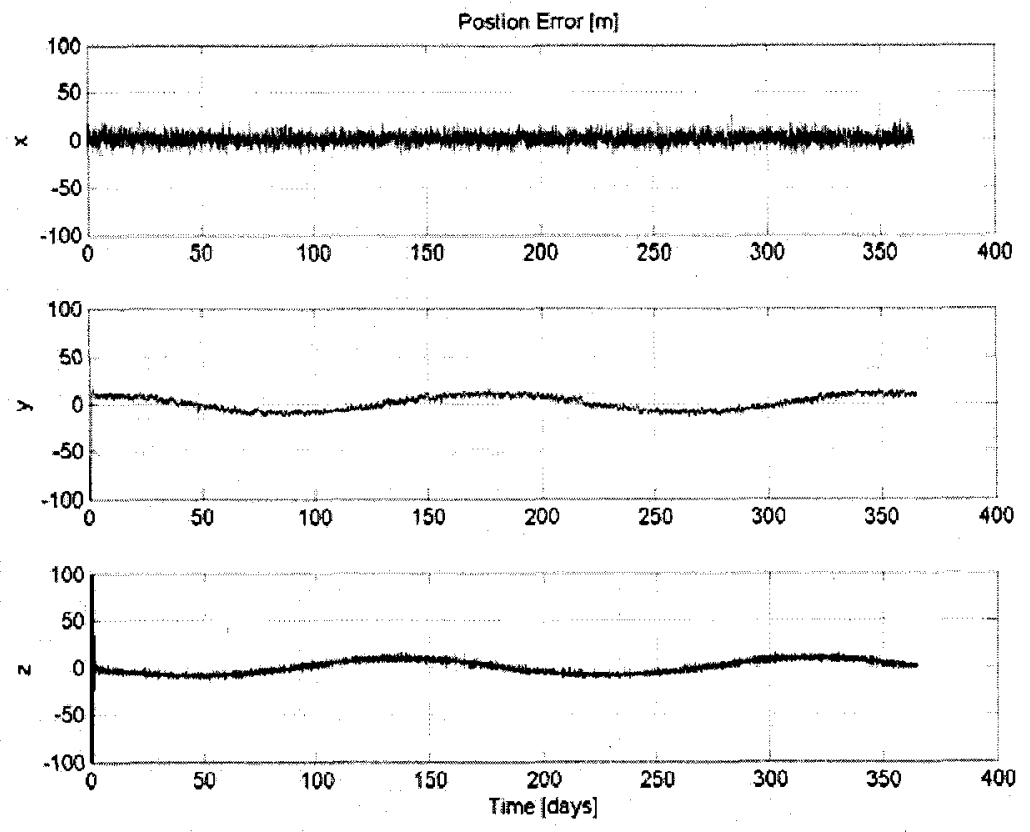

Figure 6.26 - PID position errors for 1 year (worst case) 

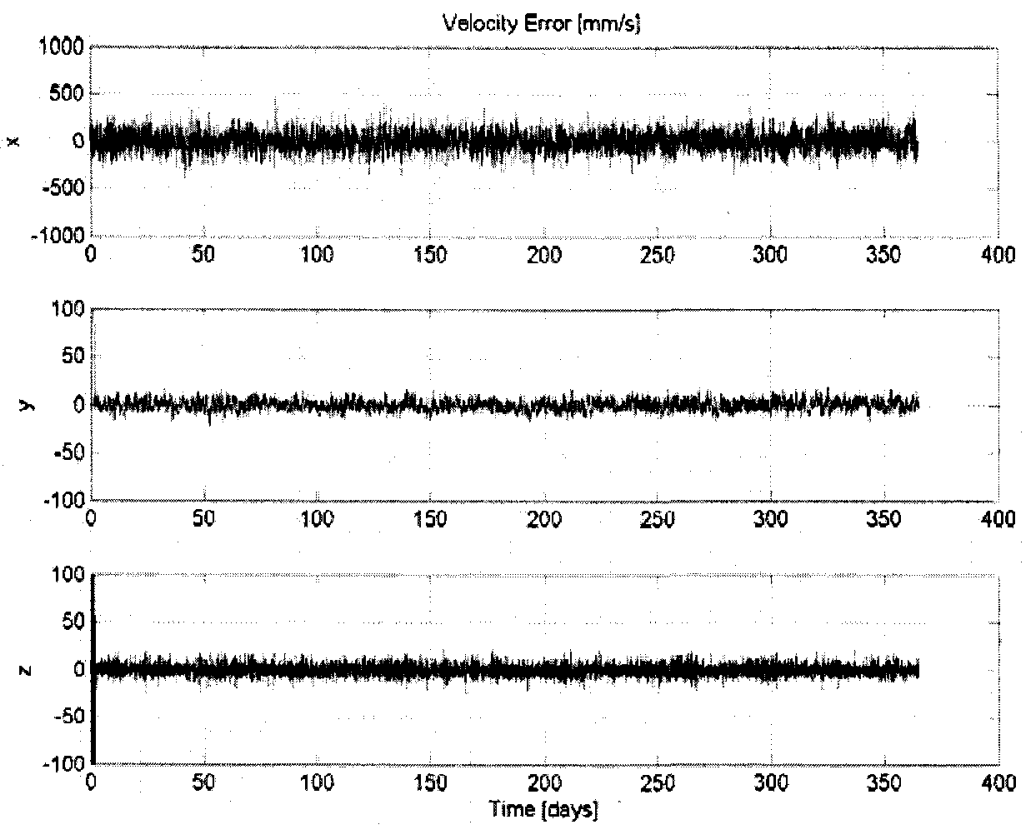

Figure 6.27 - PID velocity errors for 1 year (worst case)

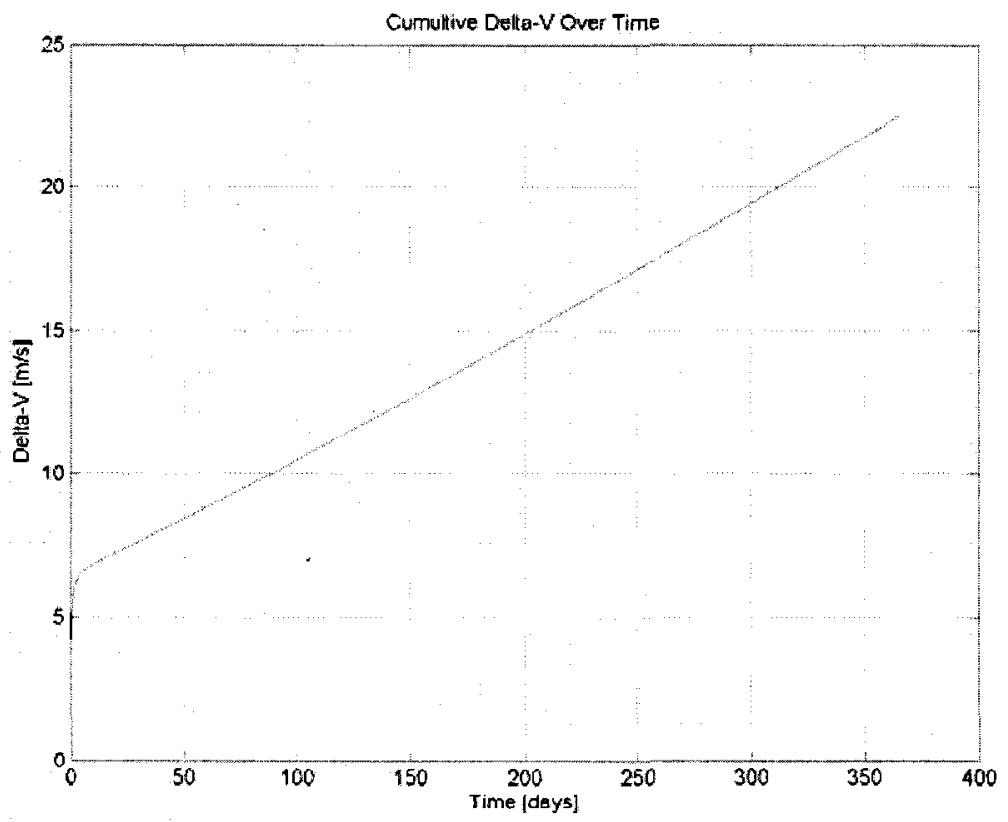

Figure 6.28 - PID $\Delta v$ profile for 1 year (worst case) 


\begin{tabular}{|c|c|c|c|c|}
\hline & \multicolumn{3}{|c|}{ Maximum Errors } & $2 \times$ \\
\hline & $x=$ & $y$ & 2 & matos \\
\hline position [m] & 27 & 17 & 15 & \\
\hline velocity $[\mathrm{mm} / \mathrm{s}]$ & 463 & 21 & 28 & 22.5 \\
\hline
\end{tabular}

Table 6.9 - PID results for 1 year (solar radiation pressure)

The results yielded are what is expected from combining all the disturbances. Characteristics from the measurement noise are present in the form of large errors and the differing initial conditions cause a large initial increase of $\Delta v$ which decreases once the error tolerances are met. With the worst case presented, PID control does not satisfy mission requirements for the given lissajous orbit.

\subsection{Summary}

\begin{tabular}{|c|c|c|c|c|}
\hline & \multicolumn{3}{|c|}{ Max Position Errors $[\mathrm{m}]$} & \multirow{2}{*}{ 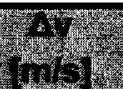 } \\
\hline & $\mathbf{x}$ & y & $2=1$ & \\
\hline Unperturbed & 0.55 & 9.3 & 8.4 & 1.46 \\
\hline Thr. Misalign. & 0.55 & 9.4 & $\overline{8.4}$ & 1.46 \\
\hline Thr. Blas & 0.58 & 9.8 & 8.8 & 1.46 \\
\hline Self-Gravity & 0.56 & $\overline{9.3}$ & 8.4 & 1.46 \\
\hline Supiter Gravity & 0.55 & $\overline{9.3}$ & 8.4 & 1.46 \\
\hline SRP & 0.55 & 9.3 & 8.4 & 1.43 \\
\hline Meas. Nolse & 48 & 17 & 15 & $\overline{36.4}$ \\
\hline Differing $1 \mathrm{Cs}_{\mathrm{s}}$ & 0.56 & $\overline{9.3}$ & $\overline{8.4}$ & $\overline{17.2}$ \\
\hline Worst Case & 27 & 17 & 15 & 22.5 \\
\hline
\end{tabular}

Table 6.10 - PID results (summary)

A summary of maximum position errors and $\Delta v$ for each case is shown in Table 6.10.

PID control is robust against all disturbances except for measurement noise and differing initial conditions. Measurement noise is noticed to corrupt the system beyond mission tolerance requirements, causing positional errors to severely increase, in turn increasing 
$\Delta \mathrm{v}$. Differing initial conditions, although maintaining positioned requirements, raises $\Delta \mathrm{v}$ significantly. The worst case scenario, however, does not witness the worst $\Delta v$ due some negation of perturbations. With the cases presented, PID is not seen to be robust enough to maintain a stable orbit with a minimal $\Delta \mathrm{v}$. 


\section{CHAPTER 7}

\section{LINEAR QUADRATIC REGULATOR}

\section{$\underline{7.1 \text { Fundamental Equations }}$}

LQR is a linear optimal control technique based on the Riccati equation, which uses weighting matrices to apply appropriate correction towards control effort or error decay. Using the linear system of the CR3BP described in Equation (3.19), the cost function of the system becomes

$$
J=\frac{1}{2} \int_{0}^{t_{f}}\left[\mathbf{x}^{T}(t) \mathbf{Q} \mathbf{x}(t)+\mathbf{u}^{T}(t) \mathbf{R u}(t)\right] d t
$$

where

$$
\begin{aligned}
& \mathbf{Q}=\operatorname{diag}\left(\mathbf{q}_{n}\right)>0 \\
& \mathbf{R}=\operatorname{diag}\left(\mathbf{r}_{m}\right)>0
\end{aligned}
$$

Here, $\mathbf{Q}$ and $\mathbf{R}$ are the weighting matrices, $\mathbf{q}$ and $\mathbf{r}$ are arrays with $n$ and $m$ elements, where $n$ is the number of states and $m$ is the number of inputs. $\mathbf{Q}$ is the weighting matrix for error decay, while $\mathbf{R}$ is the weighting matrix for control effort. The control law to minimize $J$ is given by

$$
\mathbf{u}=-\mathbf{K x}
$$


where

$$
\mathbf{K}=\mathbf{R}^{-1} \mathbf{B}^{T} \mathbf{S}
$$

and

$$
\mathbf{S A}+\mathbf{A}^{T} \mathbf{S}-\mathbf{S B R}^{-1} \mathbf{B}^{T} \mathbf{S}+\mathbf{Q}=0
$$

Equation (7.5) is the steady-state Riccati equation, which places the poles of the linear closed-loop system at $\lambda$, such that

$$
\lambda=\operatorname{eig}(\mathbf{A}-\mathbf{B K})
$$

Combining the control law with Figure 5.1, the simplified block diagram is shown in Figure 7.1 .

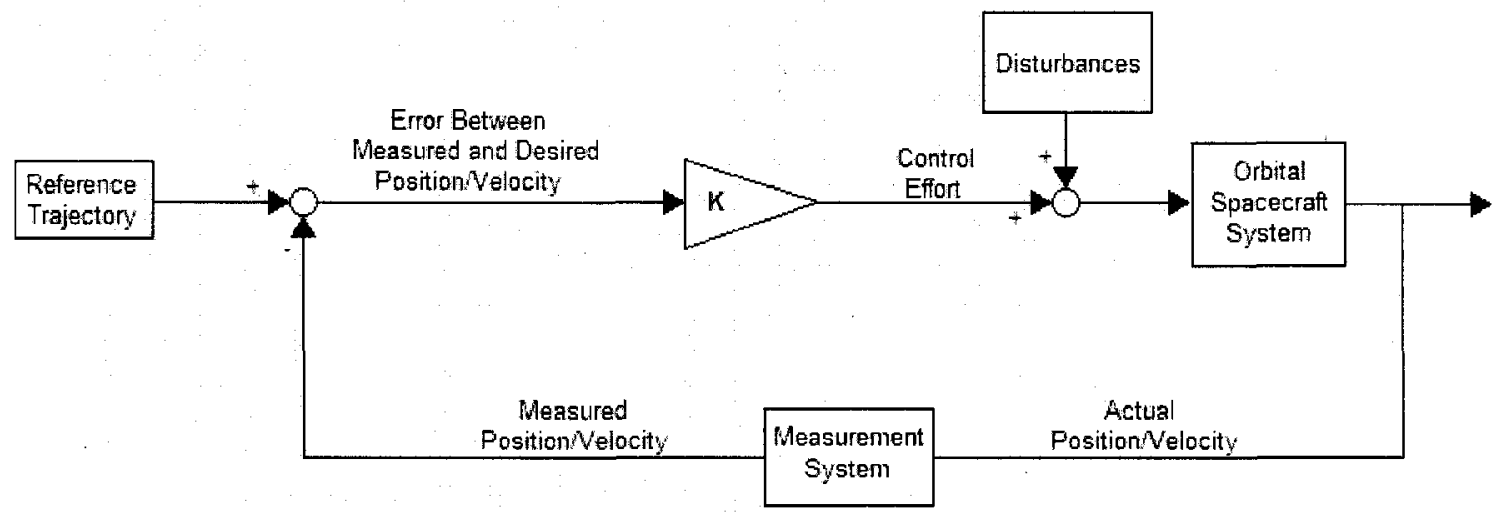

Figure 7.1 - Simplified LQR block diagram

\subsection{Gain Numbers and Closed-Loop Poles}

Like the PID method of finding gains, LQR control is applied to the linearized CR3BP system initially. The gains of $\mathbf{Q}$ and $\mathbf{R}$ are selected through analysis of the closed-loop 
poles provided by the Riccati equation and the transient response until an acceptable transient response is obtained. The gains are then implemented with the nonlinear equations and tuned with intuitive trial and error until the following gains are finalized:

$$
\begin{aligned}
\mathbf{Q} & =\left[\begin{array}{cccccc}
8 \cdot 10^{-3} & 0 & 0 & 0 & 0 & 0 \\
0 & 4 \cdot 10^{-7} & 0 & 0 & 0 & 0 \\
0 & 0 & 2 \cdot 10^{-4} & 0 & 0 & 0 \\
0 & 0 & 0 & 9 \cdot 10^{-9} & 0 & 0 \\
0 & 0 & 0 & 0 & 3 \cdot 10^{-9} & 0 \\
0 & 0 & 0 & 0 & 0 & 9 \cdot 10^{-6}
\end{array}\right] \\
\mathbf{R} & =\left[\begin{array}{ccc}
8 \cdot 10^{6} & 0 & 0 \\
0 & 10^{3} & 0 \\
0 & 0 & 10^{6}
\end{array}\right]
\end{aligned}
$$

which yields an $\mathrm{LQR}$ control gain, $\mathbf{K}$, such that

$$
\mathbf{K}=\left[\begin{array}{cccccc}
3.1 \cdot 10^{-5} & -5.4 \cdot 10^{-10} & 0 & 7.9 \cdot 10^{-3} & 2.4 \cdot 10^{-7} & 0 \\
6.9 \cdot 10^{-6} & 2 \cdot 10^{-5} & 0 & 1.9 \cdot 10^{-3} & 6.3 \cdot 10^{-3} & 0 \\
0 & 0 & 1.4 \cdot 10^{-5} & 0 & 0 & 5.3 \cdot 10^{-3}
\end{array}\right]
$$

and places the linear closed-loop poles at

$$
\lambda=\left[\begin{array}{c}
-4 \cdot 10^{-3} \pm 4 \cdot 10^{-3} i \\
-3.1 \cdot 10^{-3} \pm 3.1 \cdot 10^{-3} i \\
-2.7 \cdot 10^{-3} \pm 2.7 \cdot 10^{-3} i
\end{array}\right]
$$

\subsection{Unperturbed System Results}

Results pertaining to the LQR controller applied to the nonlinear system without disturbances or perturbations are shown in Figures 7.2 through 7.4. 

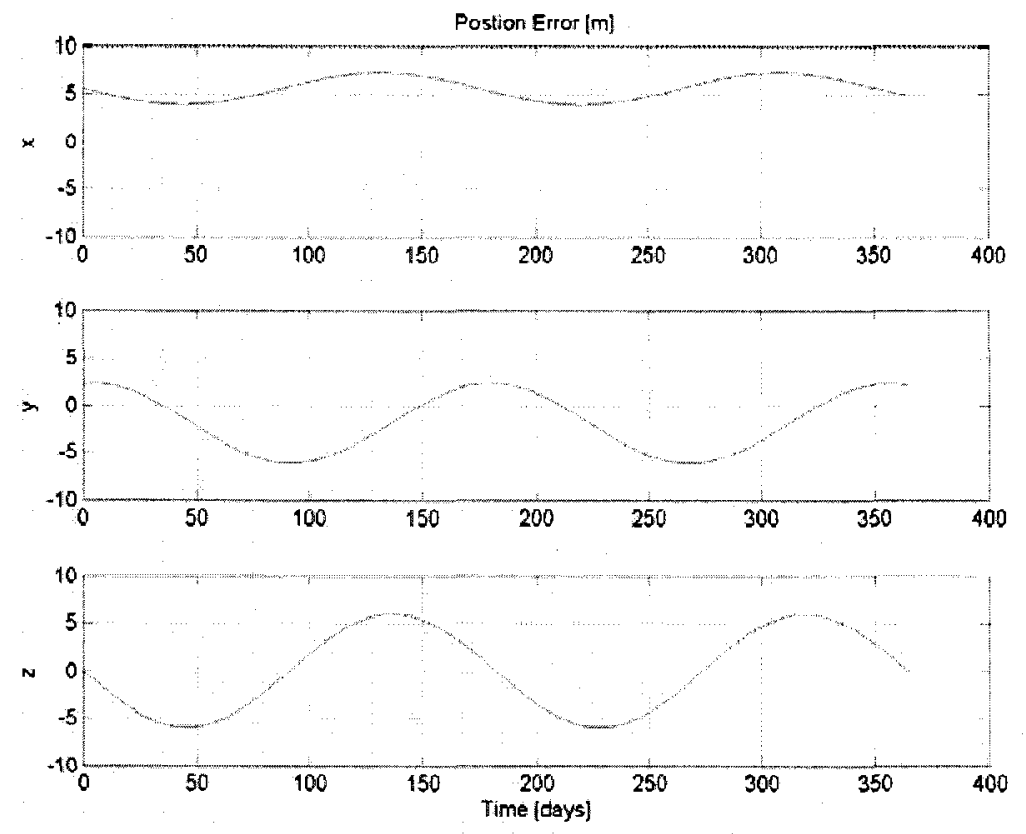

Figure 7.2 - LQR position errors for 1 year (unperturbed)
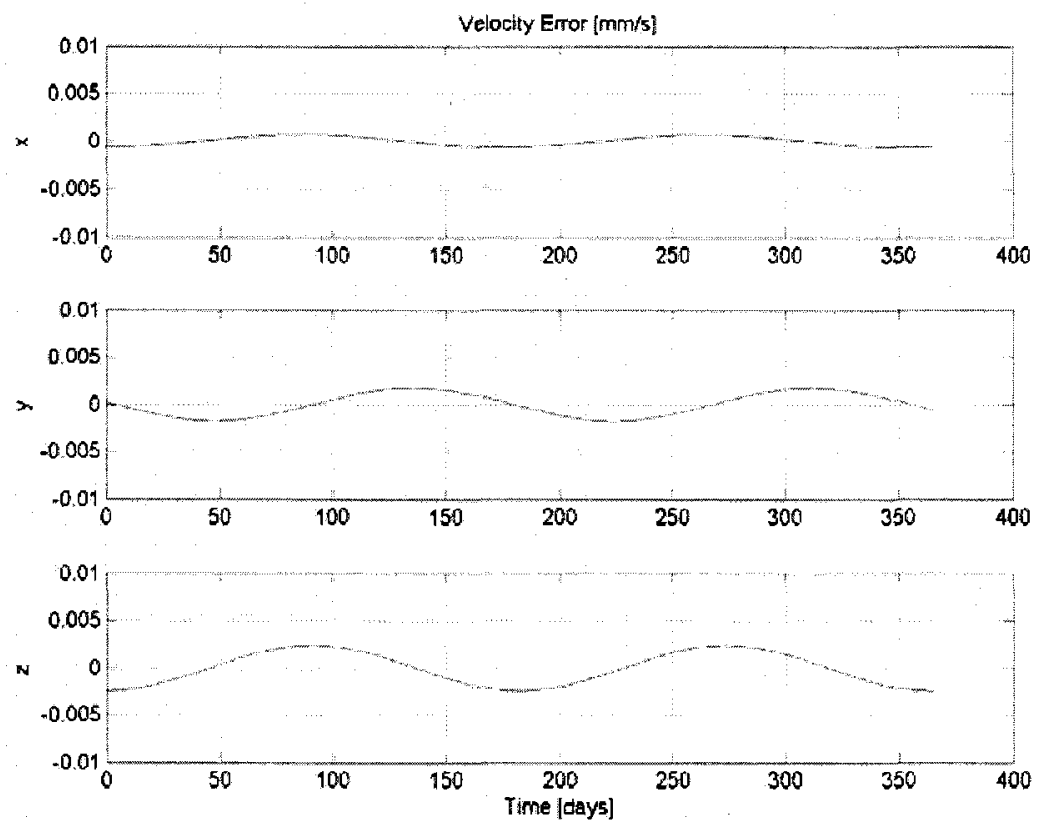

Figure 7.3 - LQR velocity errors for 1 year (unperturbed) 


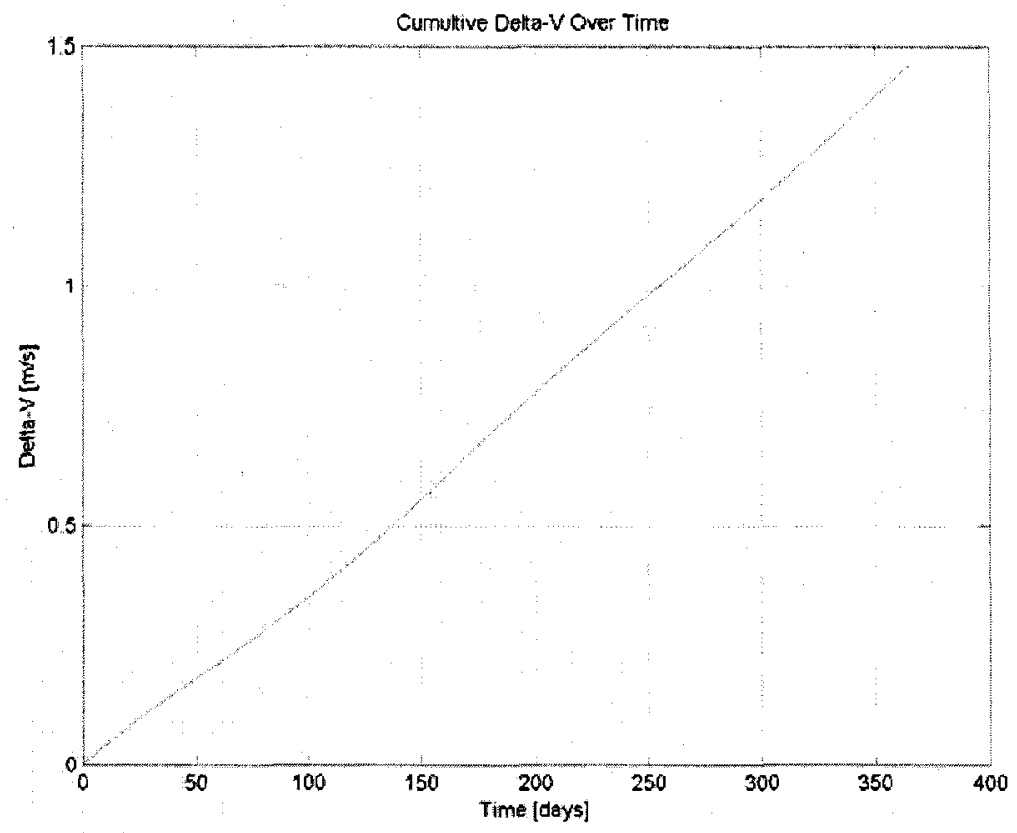

Figure 7.4 - LQR $\Delta v$ profile for 1 year (unperturbed)

\begin{tabular}{|c|c|c|c|c|}
\hline & \multicolumn{3}{|c|}{ Maximum Errors } & \multirow{2}{*}{$\begin{array}{l}\text { Wy } \\
\text { me }\end{array}$} \\
\hline & $x=$ & 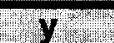 & 2 & \\
\hline position $[\mathrm{m}]$ & 7.2 & 6.1 & 5.9 & \\
\hline velocity $[\mu \mathrm{m} / \mathrm{s}]$ & 0.069 & 1.8 & 2.4 & 1.40 \\
\hline
\end{tabular}

Table 7.1 - LQR results for 1 year (unperturbed)

Table 7.1 lists the $\Delta \mathrm{v}$ and maximum errors for position and velocity once the orbit is stabilized. The controller performs well, maintaining errors within the allowable tolerances. The $\mathrm{x}$-position error is maintained but produces a bias of five meters. Decreasing this bias would require a larger control effort, although the bias is allowable since the error remains with in the absolute tolerance. As such, the bias is not corrected for the sake of fuel effiency. The y- and z-position errors exhibit larger oscillations but remain within the ten meter allowance. This is left as is for the same reason as that of the bias. Initial velocity errors exist in all three coordinates and follows the same reasons 
described as with the PID controller, since velocity errors are not a mission requirement. Most importantly, though, $\Delta \mathrm{v}$ is kept to a minimal value while maintaining the trajectory.

\subsection{Thruster Misalignment Results}

Results pertaining to the LQR controller applied to the nonlinear system with thruster misalignments of a tenth of a degree are shown in Figures 7.5 through 7.7. Numerical results are listed in Table 7.2.
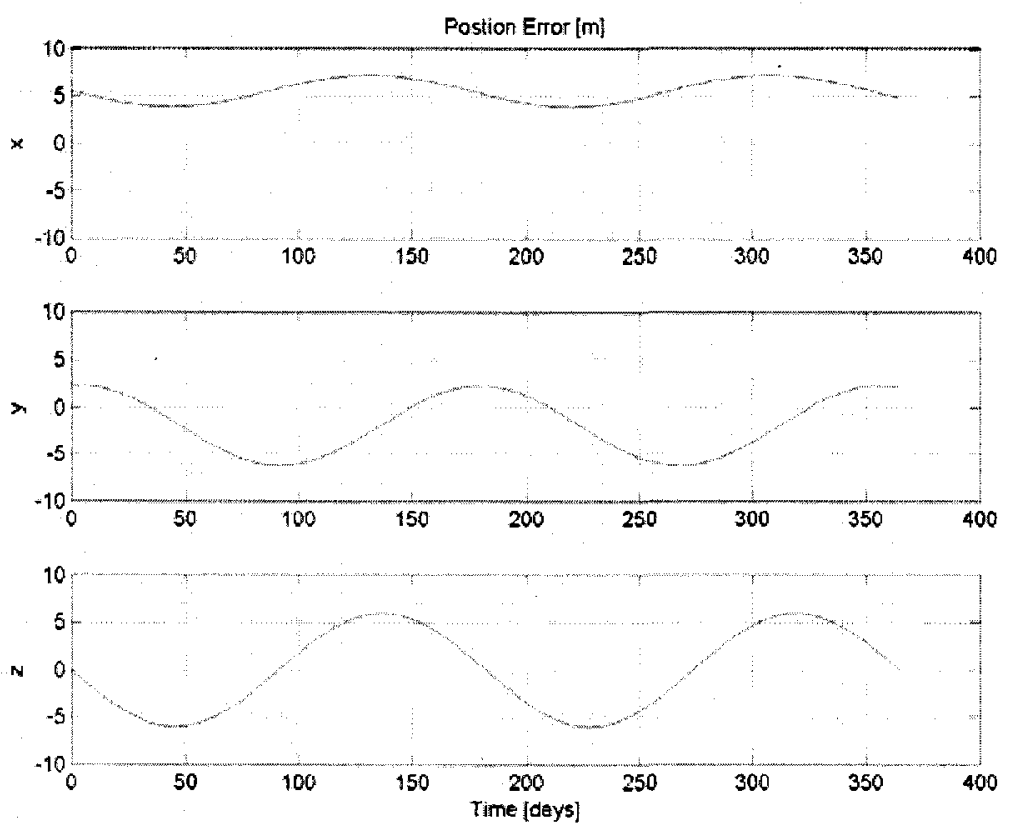

Figure 7.5 - LQR position errors for 1 year (thruster misalignments) 

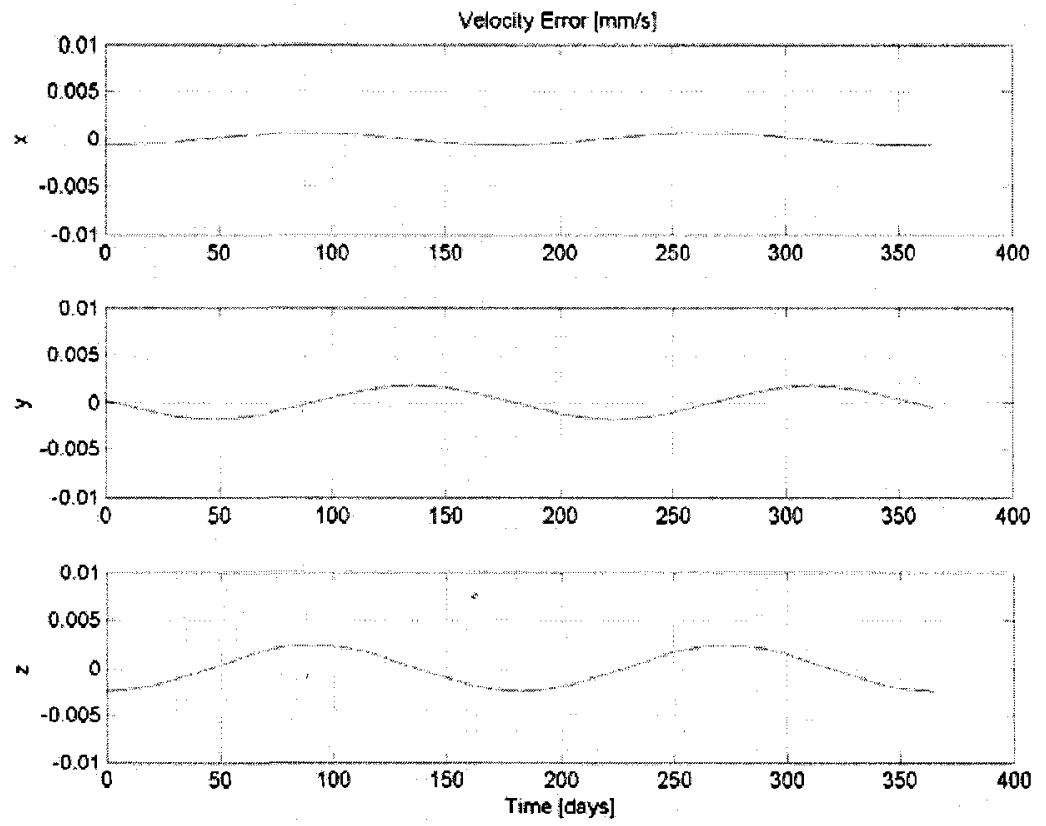

Figure 7.6 - LQR velocity errors for 1 year (thruster misalignments)

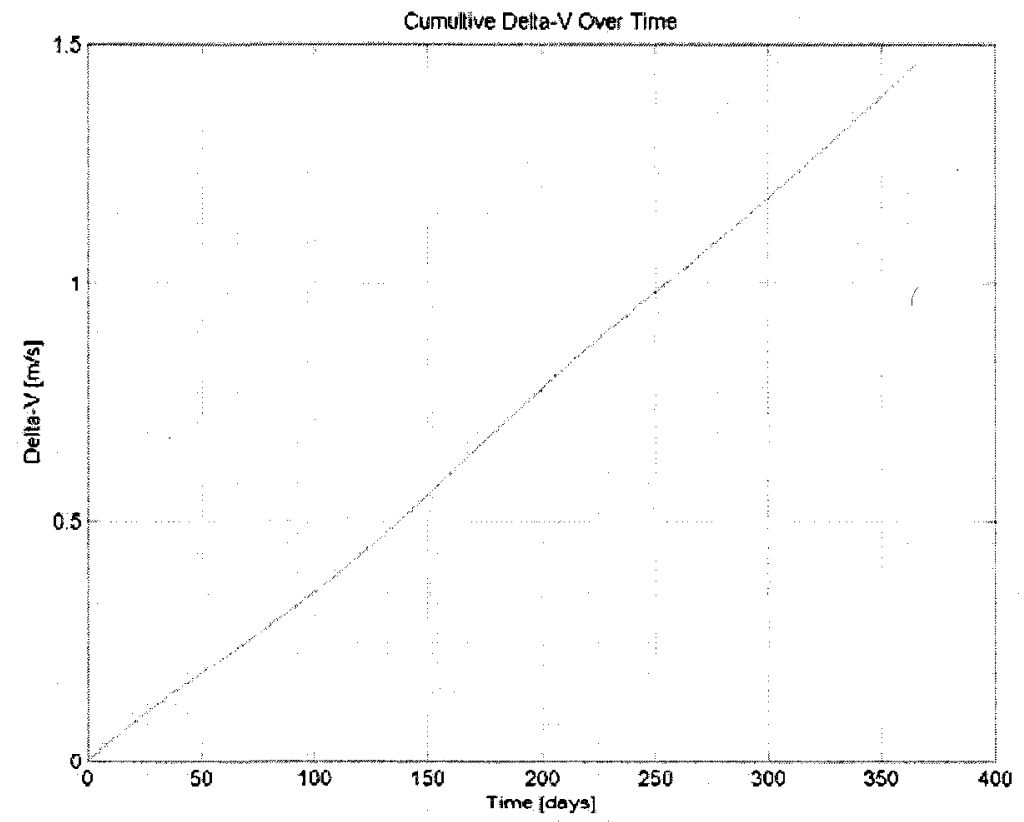

Figure 7.7 - LQR $\Delta \mathrm{v}$ profile for 1 year (thruster misalignments) 


\begin{tabular}{|c|c|c|c|c|}
\cline { 2 - 5 } \multicolumn{1}{c|}{} & \multicolumn{3}{c|}{ Maximum Errors } & \\
\cline { 2 - 5 } & $\mathbf{X}$ & $\mathbf{y}$ & $\mathbf{z}$ & \\
\hline position [m] & 7.7 & 6.1 & 5.9 & 1.46 \\
\hline velocity [um]s] & 0.069 & 1.8 & 2.4 & 1.4 \\
\hline
\end{tabular}

Table 7.2 - LQR results for 1 year (thruster misalignments)

The controller performs well, maintaining the same errors as the unperturbed system and maintaining the minimal $\Delta \mathrm{v}$. The $\mathrm{x}$-error is slightly increased but is not of concern since it is still with the allowed tolerance. The LQR controller is shown to not be affected in the presence of thruster misalignments.

\subsection{Thruster Bias Results}

Results pertaining to the LQR controller applied to the nonlinear system with thruster biases of $-5 \%$ are shown in Figures 7.8 through 7.10. Numerical results are listed in Table 7.3. 

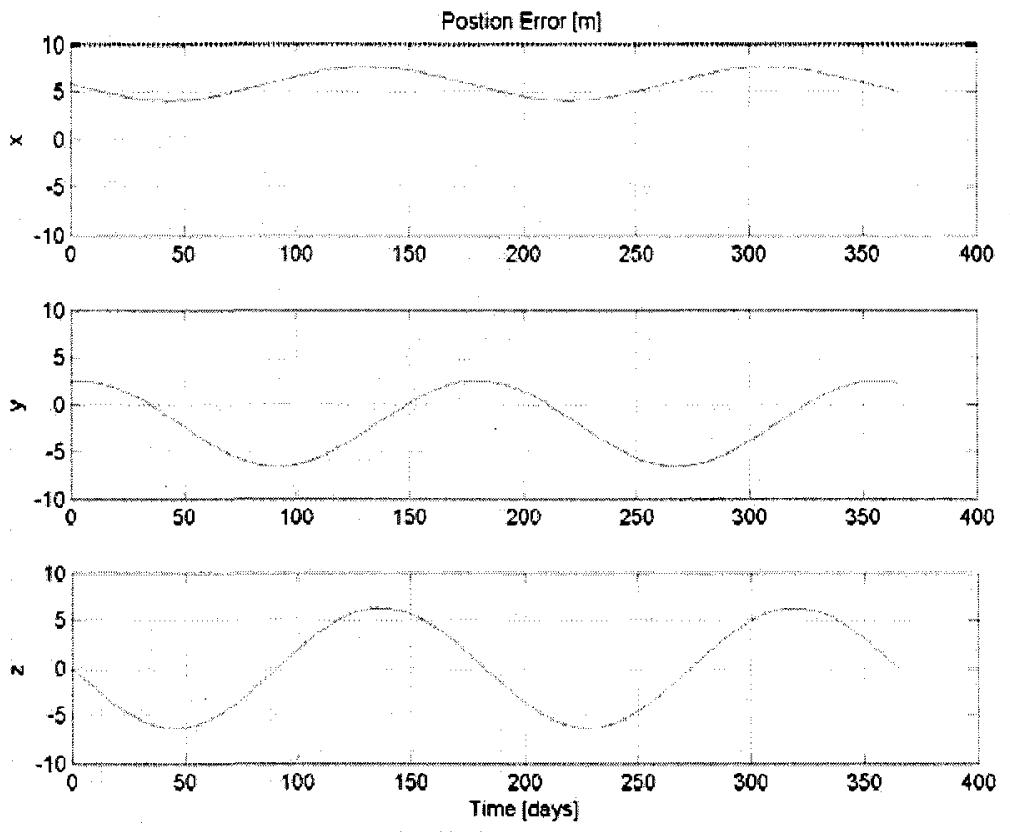

Figure 7.8 - LQR position errors for 1 year (thruster bias)
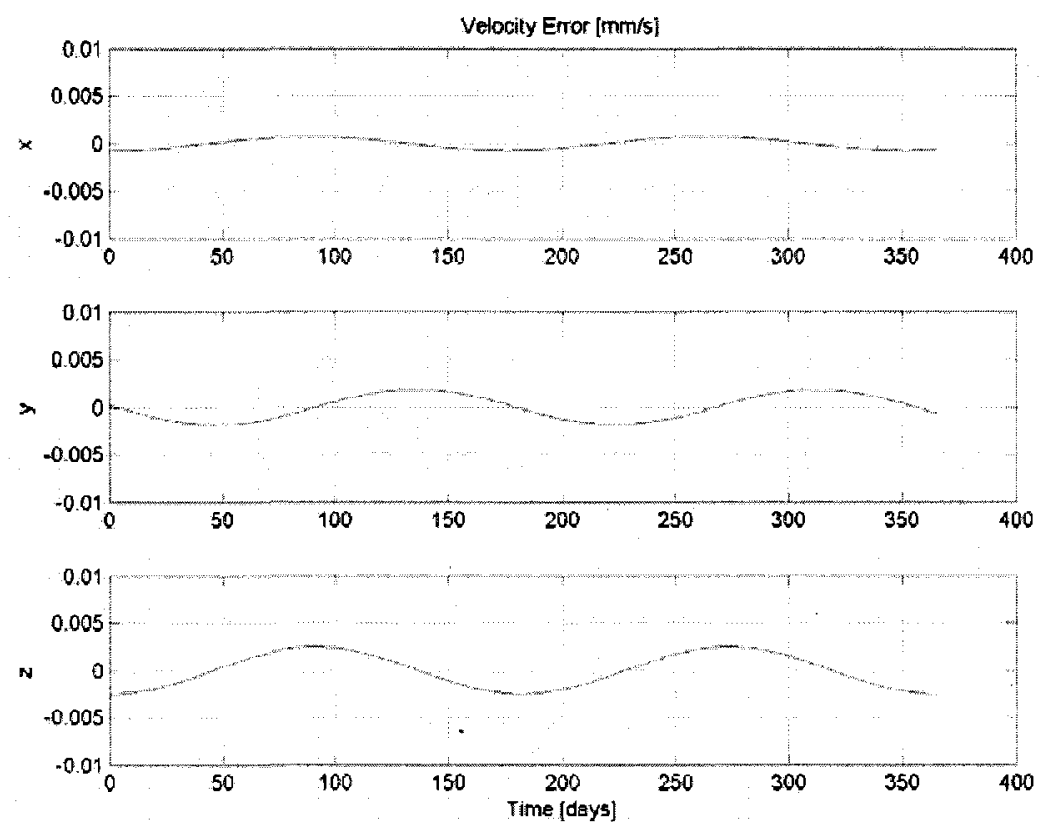

Figure 7.9 - LQR velocity errors for 1 year (thruster bias) 


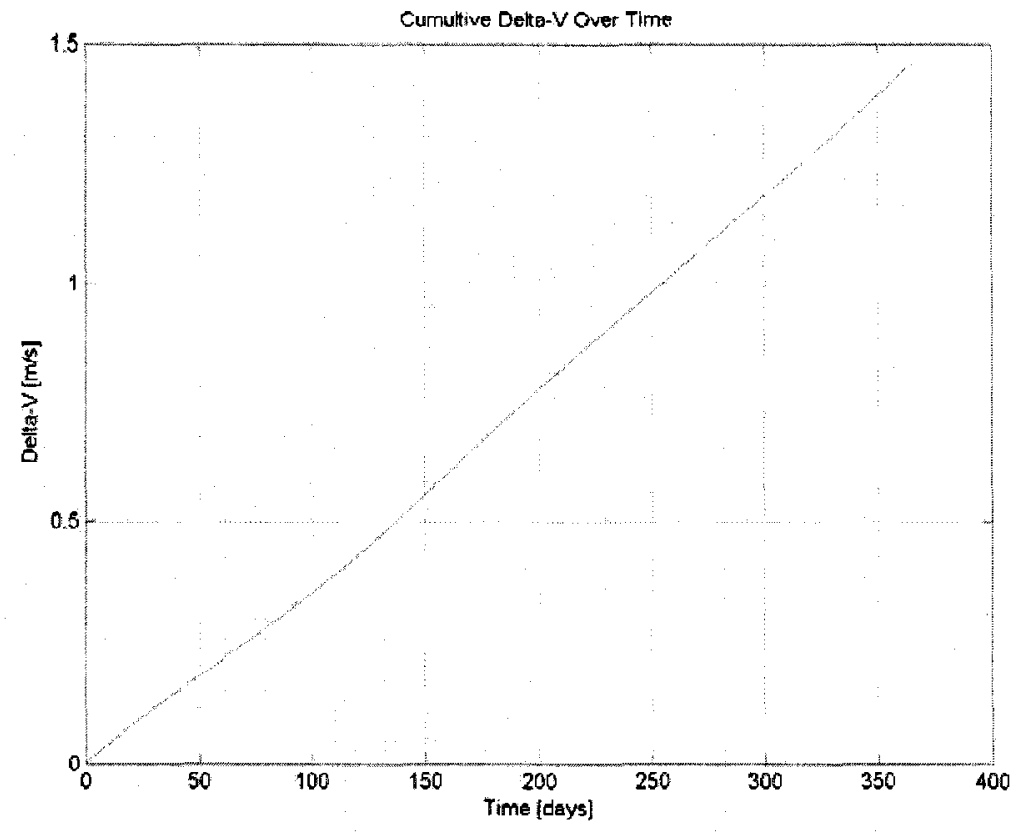

Figure 7.10 - LQR $\Delta v$ profile for 1 year (thruster bias)

\begin{tabular}{|c|c|c|c|c|}
\cline { 2 - 5 } \multicolumn{1}{c|}{} & \multicolumn{4}{c|}{ Maximum Errors } \\
\cline { 2 - 5 } \multicolumn{1}{c|}{} & $\mathbf{x}$ & $\mathbf{y}$ & $\mathbf{z}$ & \\
\hline position $[\mathrm{m}]$ & 7.6 & 6.4 & 6.2 & 1.46 \\
\hline velocity [pm/s] & 0.072 & 1.8 & 2.6 & 1.46 \\
\hline
\end{tabular}

Table 7.3 - LQR results for 1 year (thruster bias)

The controller is effective, yielding errors just slightly larger than the unperturbed system but still within the allowed tolerance. $\Delta \mathrm{v}$ is also maintained to be minimal. Thruster is seen to be negligible against $L Q R$. 


\subsection{Self-Gravity Results}

Results pertaining to the $L Q R$ controller applied to the nonlinear system with self-gravity from the leader satellite are shown in Figures 7.11 through 7.13 . Numerical results are listed in Table 7.4
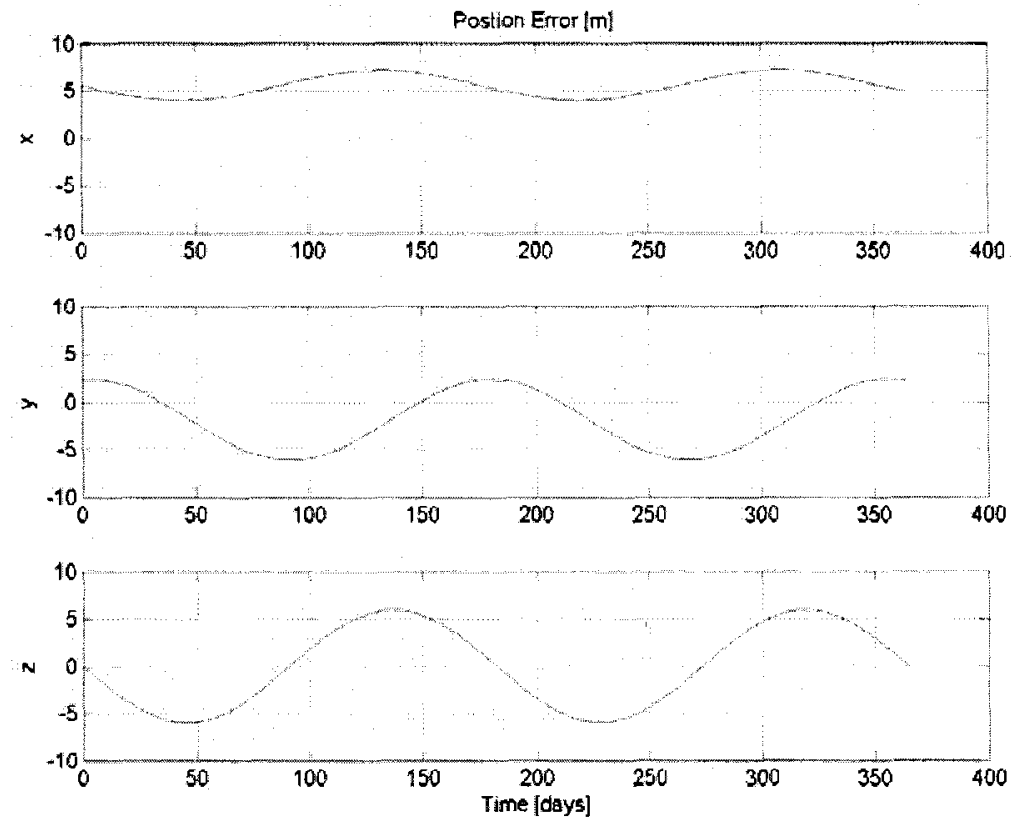

Figure 7.11 - LQR position errors for 1 year (self-gravity) 

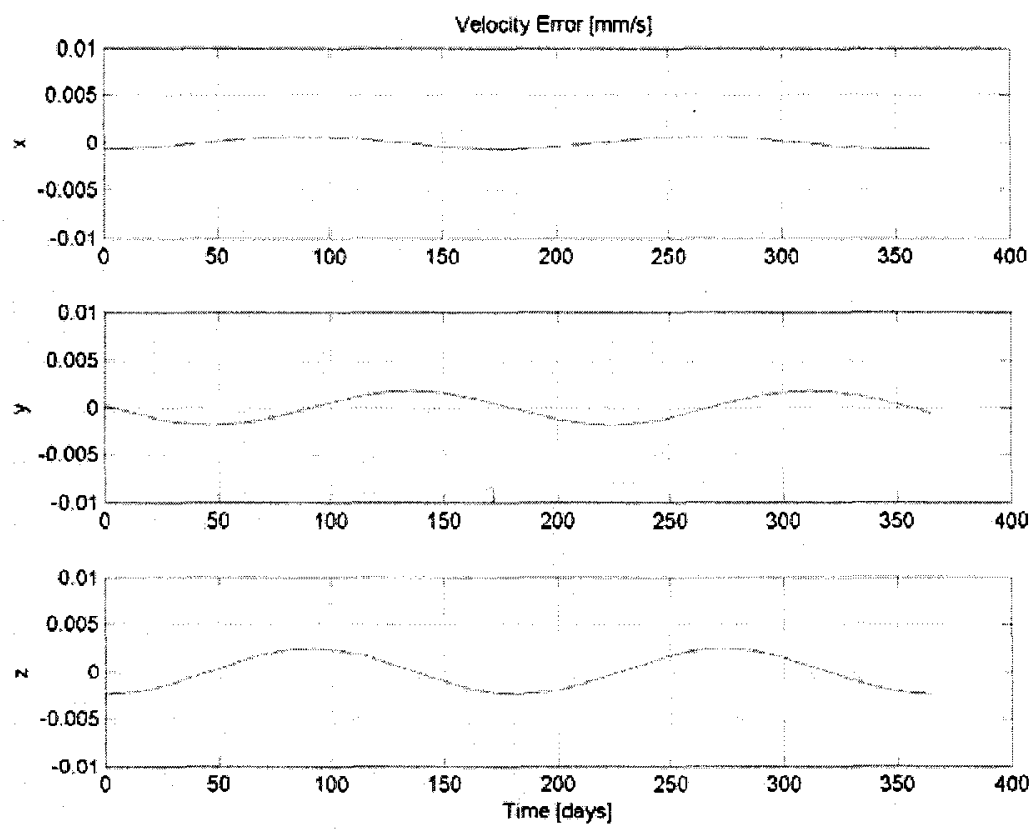

Figure 7.12 - LQR velocity errors for 1 year (self-gravity)

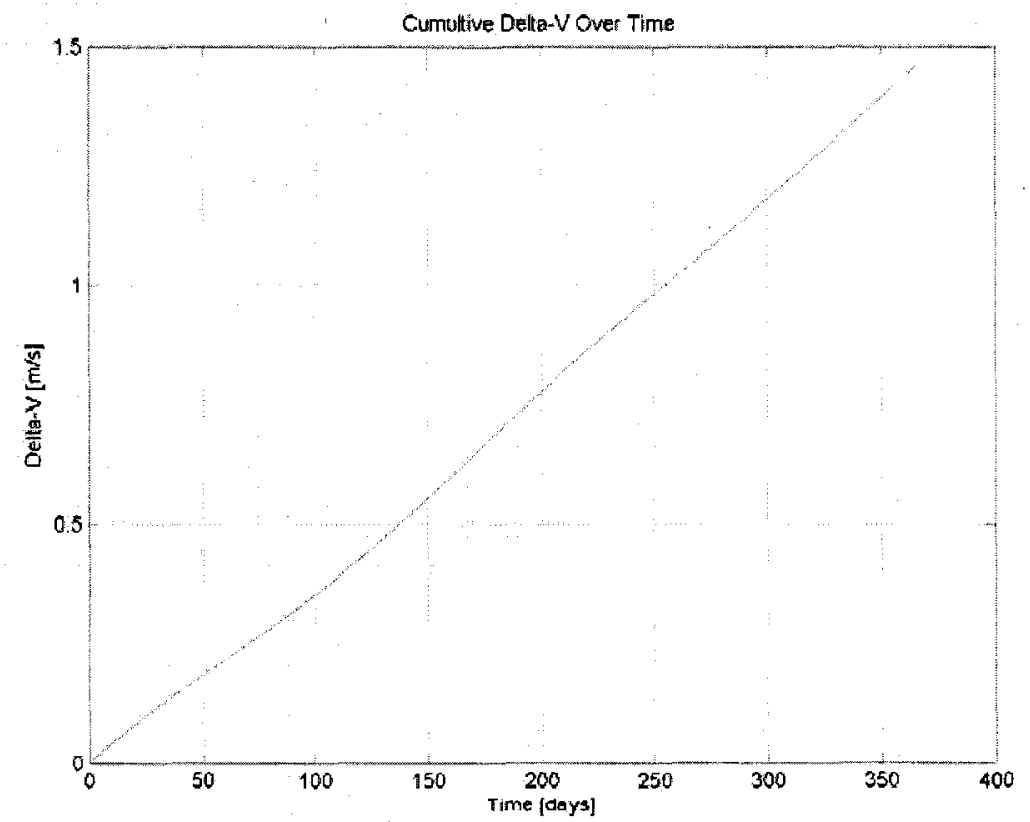

Figure 7.13 - LQR $\Delta v$ profile for 1 year (self-gravity) 


\begin{tabular}{|c|c|c|c|c|}
\hline & \multicolumn{3}{|c|}{ Maximum Errors } & $3 x+2$ \\
\hline & $x$ & $y$ & & mis! \\
\hline position [m] & 7.2 & 6.1 & 5.9 & \\
\hline velocity $[\mu \mathrm{m} / \mathrm{s}]$ & 0.069 & 1.8 & 2.4 & 1. \\
\hline
\end{tabular}

Table 7.4 - LQR results for 1 year (self-gravity)

Figures 7.11 through 7.13 show the controller produces low errors while maintaining the minimal $\Delta \mathrm{v}$. The LQR controller is shown to remain stable against self-gravity from the leader.

\subsection{Jupiter Gravity Results}

Results pertaining to the LQR controller applied to the nonlinear system while experiencing gravitational forces due to Jupiter are shown in Figures 7.14 through 7.17 . Numerical results are listed in Table 7.5. 

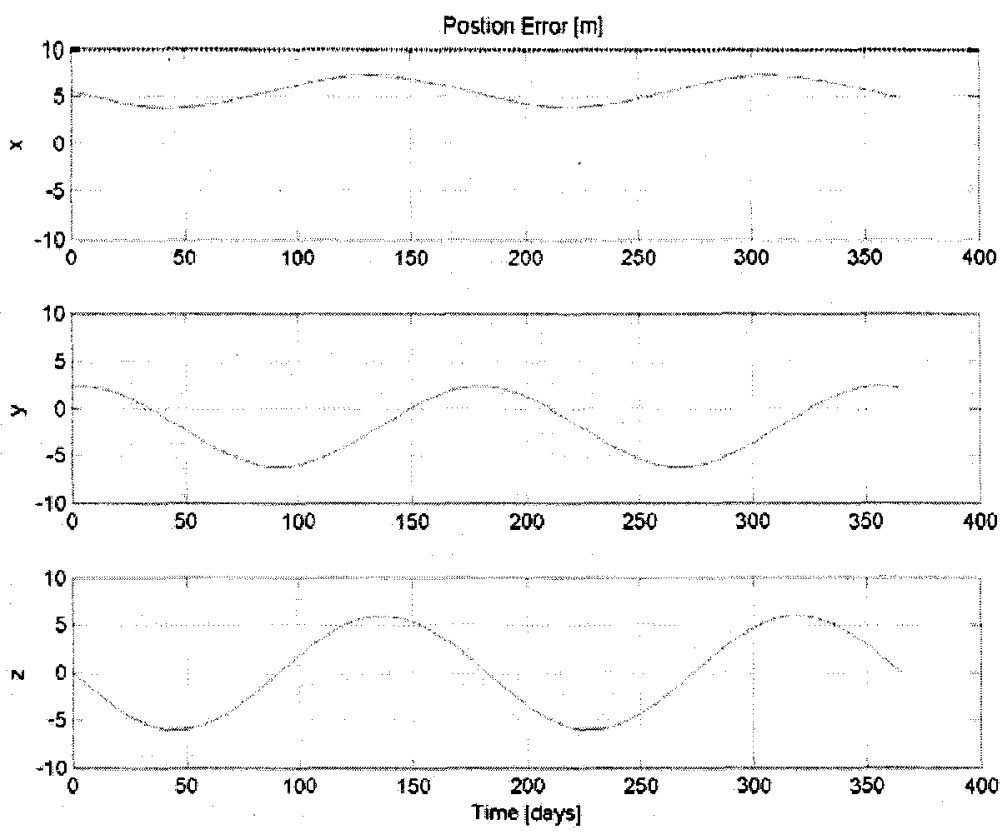

Figure 7.14 - LQR position errors for 1 year (Jupiter)
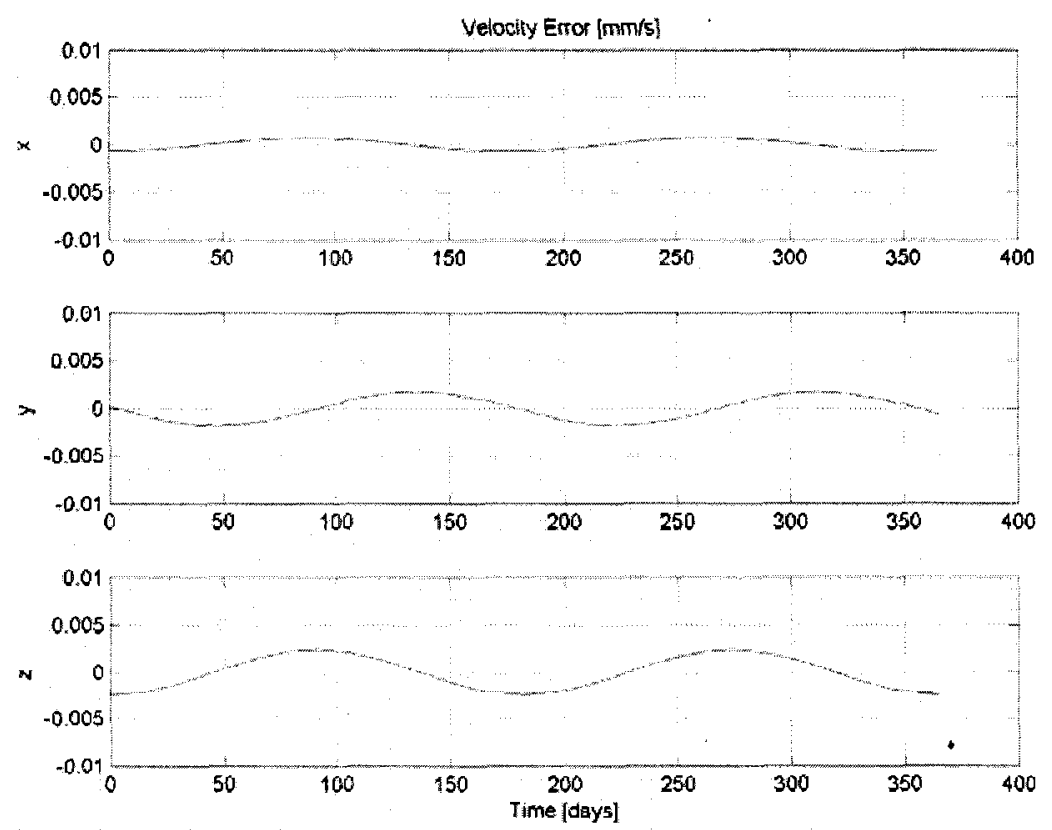

Figure 7.15 - LQR velocity errors for 1 year (Jupiter) 


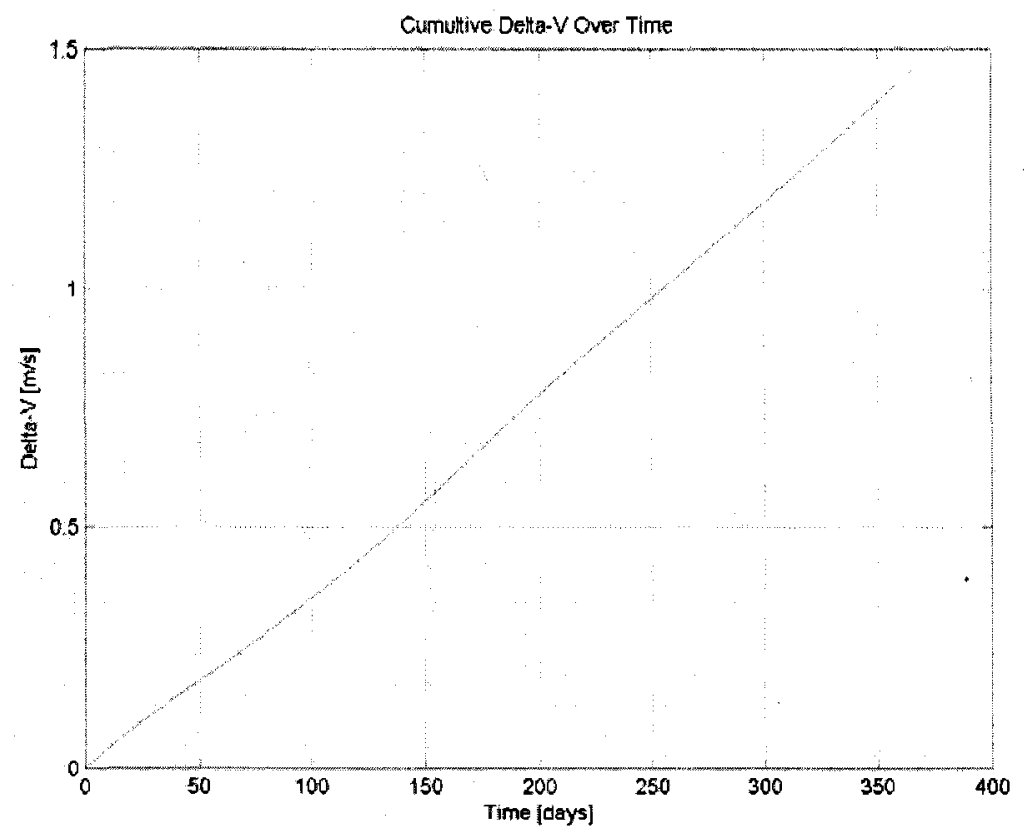

Figure 7.16 - LQR $\Delta v$ profile for 1 year (Jupiter)

\begin{tabular}{|c|c|c|c|c|}
\hline & \multicolumn{3}{|c|}{ Maximum Errors } & \multirow{2}{*}{$\begin{array}{c}4 y \\
4 \\
4\end{array}$} \\
\hline & $\mathrm{x}$ & $y$ & 2 & \\
\hline position & 7.2 & 6.1 & 5.9 & \multirow{2}{*}{1.46} \\
\hline Velocity $[\mathrm{pm} / \mathrm{s}$ & 0.069 & 1.8 & 2.4 & \\
\hline
\end{tabular}

Table 7.5 - LQR results for 1 year (Jupiter)

Once again, the controller produces results equal to those of the unperturbed system. The LQR controller is shown to maintain mission requirements in the presence of gravitational forces due to Jupiter. 


\subsection{Solar Radiation Pressure Results}

Results pertaining to the LQR controller applied to the nonlinear system with the presence of solar radiation pressure are shown in Figures 7.17 through 7.19. Numerical results are listed in Table 7.7.
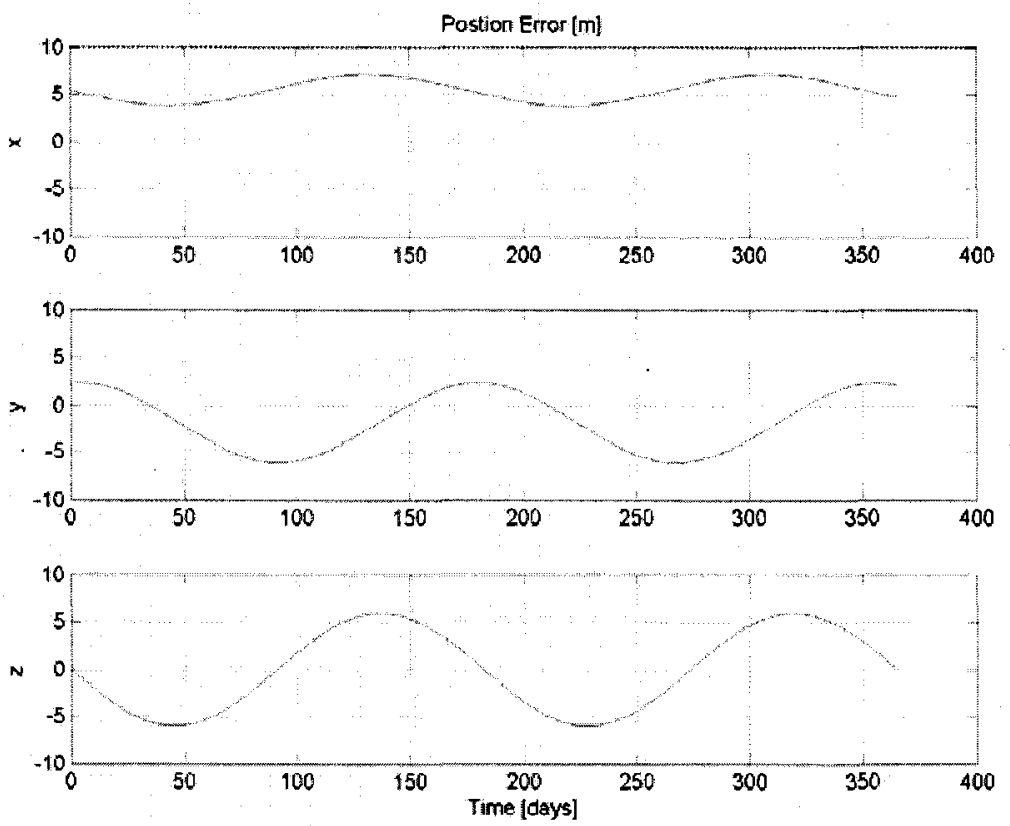

Figure 7.17 - LQR position errors for 1 year (solar radiation pressure) 

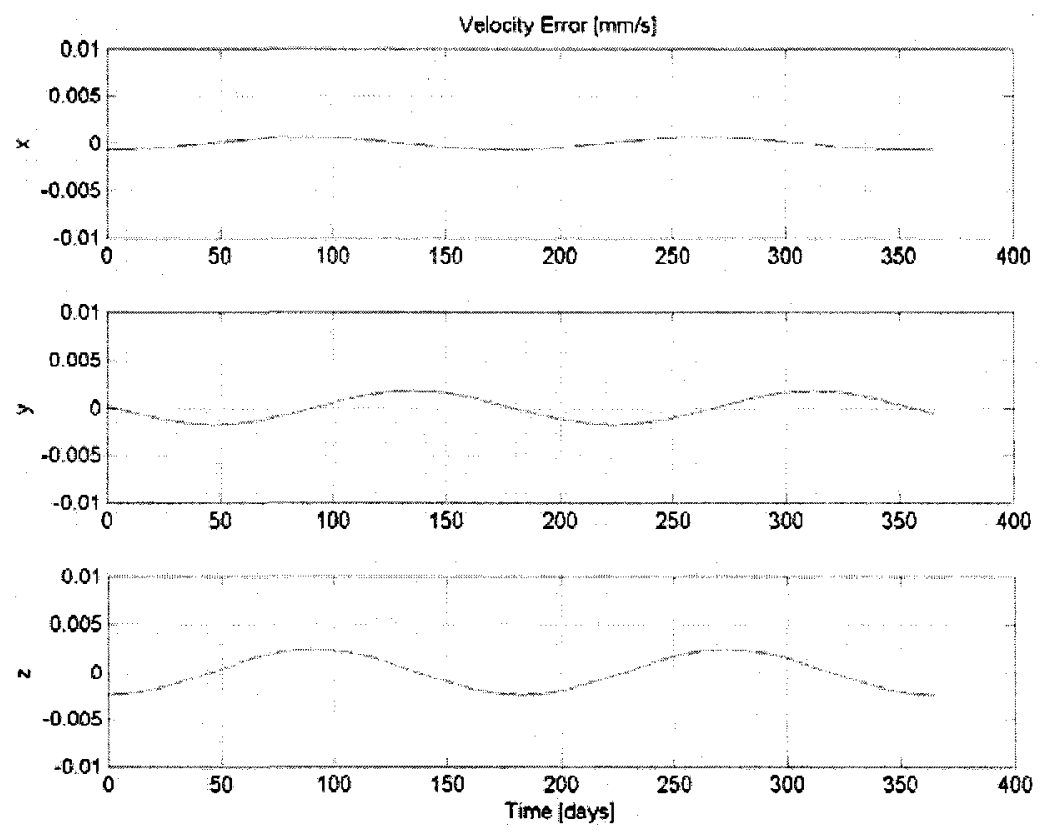

Figure 7.18 - LQR velocity errors for 1 year (solar radiation pressure)

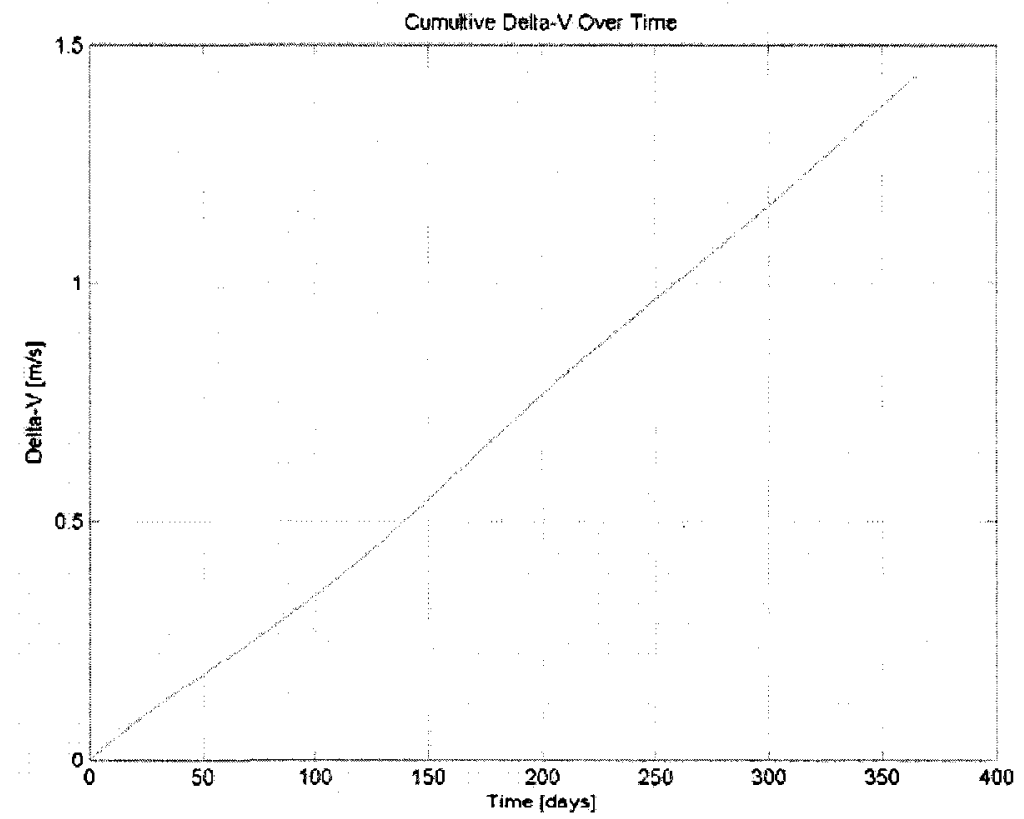

Figure 7.19 - LQR $\Delta v$ profile for 1 year (solar radiation pressure) 


\begin{tabular}{|c|c|c|c|c|}
\cline { 2 - 5 } \multicolumn{1}{c|}{} & \multicolumn{3}{c|}{ Maximum Errors } & \\
\cline { 2 - 5 } \multicolumn{1}{c|}{} & $\boldsymbol{X}$ & $\mathbf{y}$ & $\boldsymbol{z}$ & . \\
\hline position $[\mathrm{m}]$ & 7.1 & 6.1 & 5.9 & 1.43 \\
\hline velocity [um/s] & 0.069 & 1.8 & 2.4 & 1.4 \\
\hline
\end{tabular}

Table 7.6 - LQR results for 1 year (solar radiation pressure)

Table 7.6 shows the position errors are kept within the tolerance and the $\Delta v$ is minimized smaller than unperturbed system. SRP is seen to negate some nonlinearities present in the CR3BP. The LQR controller is shown to provide desirable results against solar radiation pressure.

\subsection{Measurement Noise Results}

Results pertaining to the LQR controller applied to the nonlinear system with the presence of measurement noise are shown in Figures 7.20 through 7.22. Numerical results are listed in Table 7.7. 

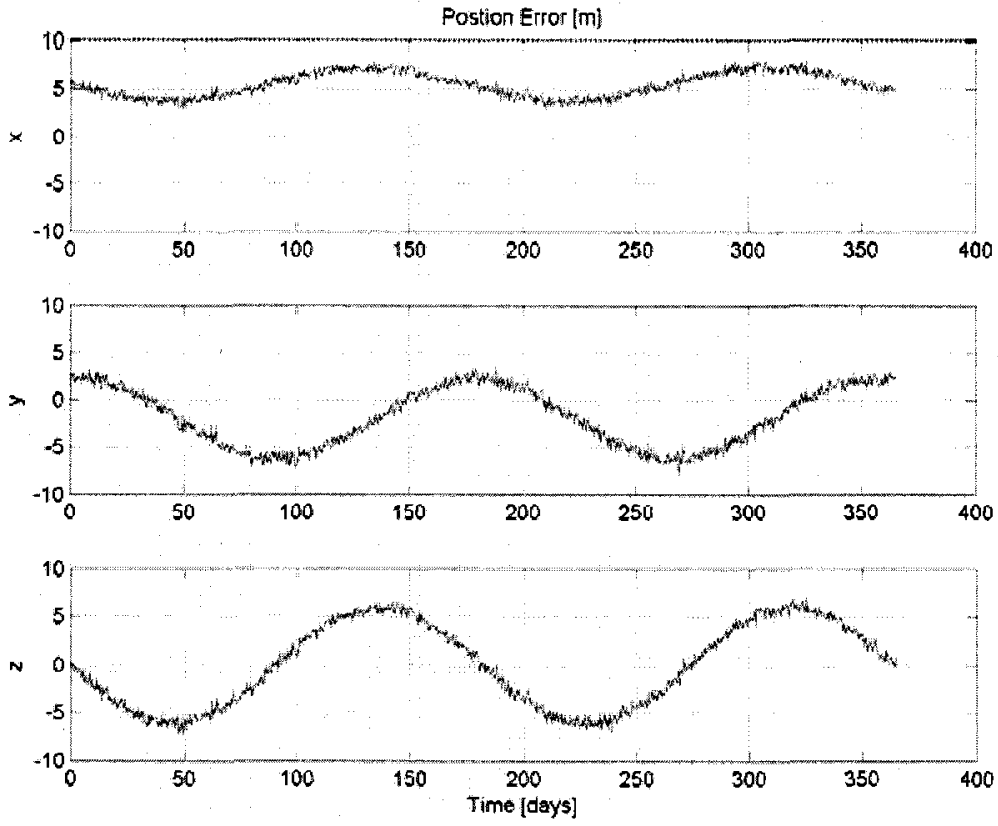

Figure 7.20 - LQR position errors for 1 year (measurement noise)
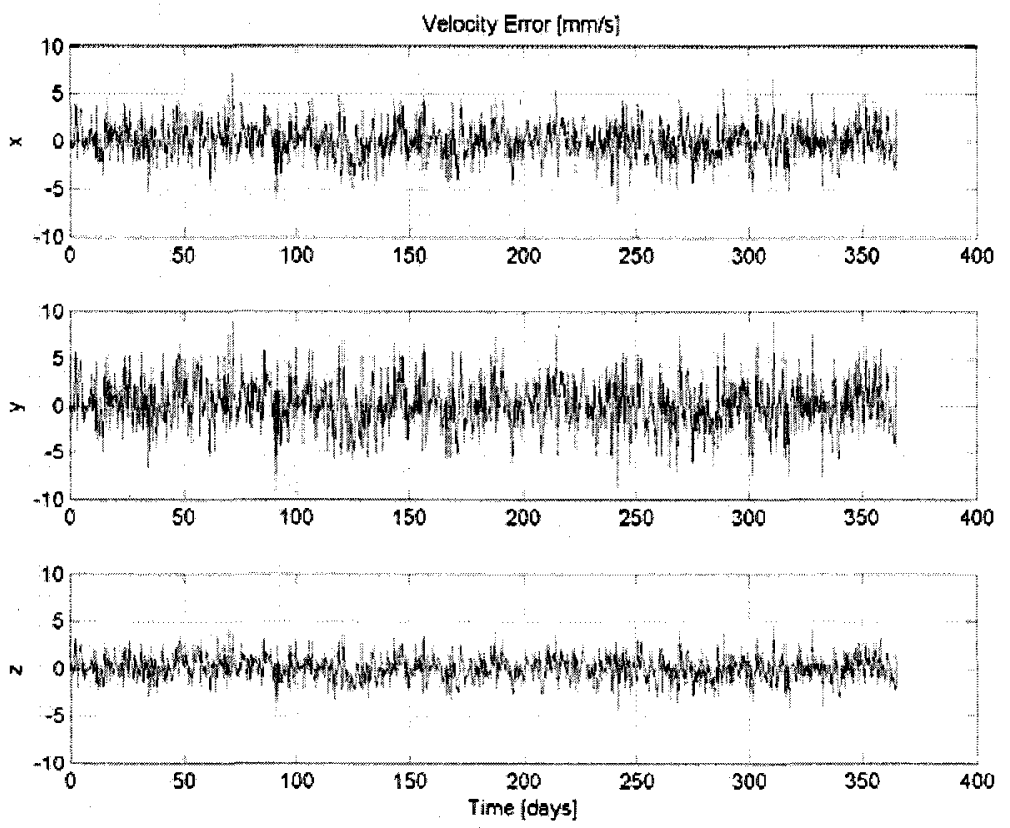

Figure 7.21 - LQR velocity errors for 1 year (measurement noise) 


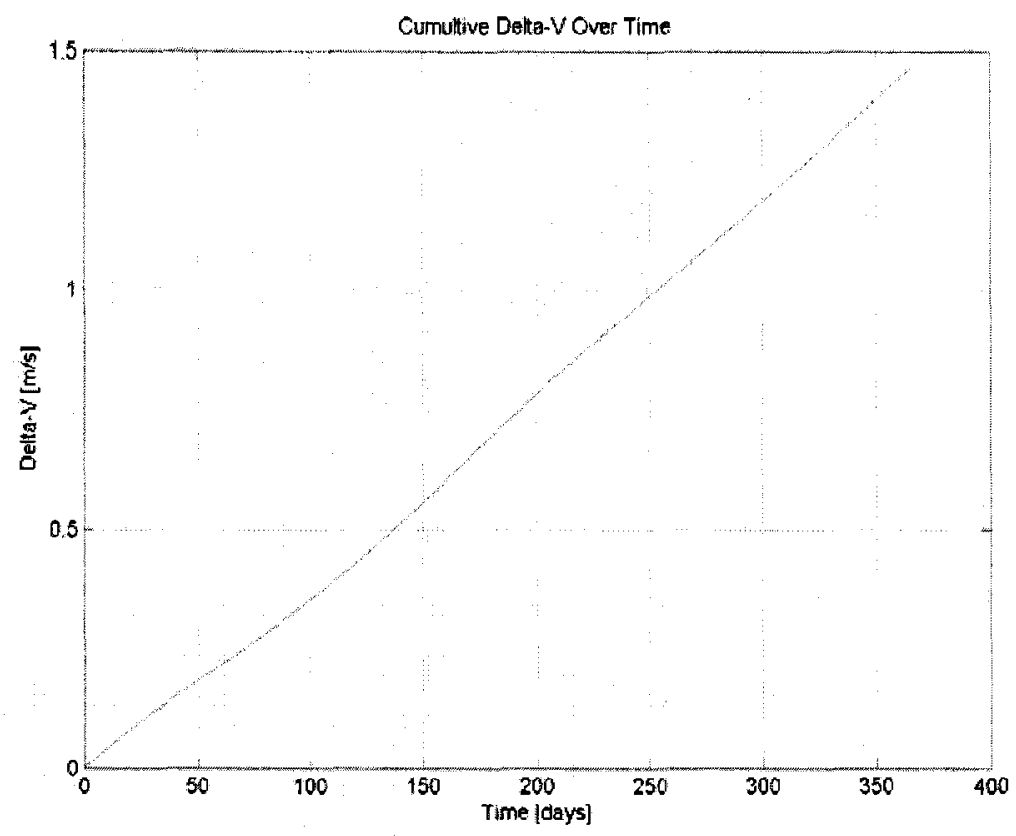

Figure 7.22 - LQR $\Delta v$ profile for 1 year (measurement noise)

\begin{tabular}{|c|c|c|c|c|}
\hline & \multicolumn{3}{|c|}{ Waximum Exrors } & \multirow{2}{*}{968} \\
\hline & $x$ & & $z$ & \\
\hline position $[\mathbf{m}]$ & 8 & 7.9 & 7.2 & \multirow{2}{*}{1.46} \\
\hline velocity [mm/s] & $\overline{7.2}$ & 9.2 & 4.6 & \\
\hline
\end{tabular}

Table 7.7 - LQR results for 1 year (measurement noise)

The resulting position errors from the measurement are slightly higher (but still within the allowable tolerance) than the unperturbed system in the presence of measurement noise. The velocity errors, however increase by a factor of 100 . But since mission requirements do not put a demand on velocity errors, this error is considered acceptable. Since the position errors are within the requirements and $\Delta v$ is maintained at a minimum, the LQR controller is considered to perform adequately in the presence of measurement noise. 


\subsection{Differing Initial Conditions Results}

Results pertaining to the LQR controller applied to the nonlinear system with incorrect initial conditions are shown in Figures 7.23 through 7.25. Numerical results are listed in Table 7.8.
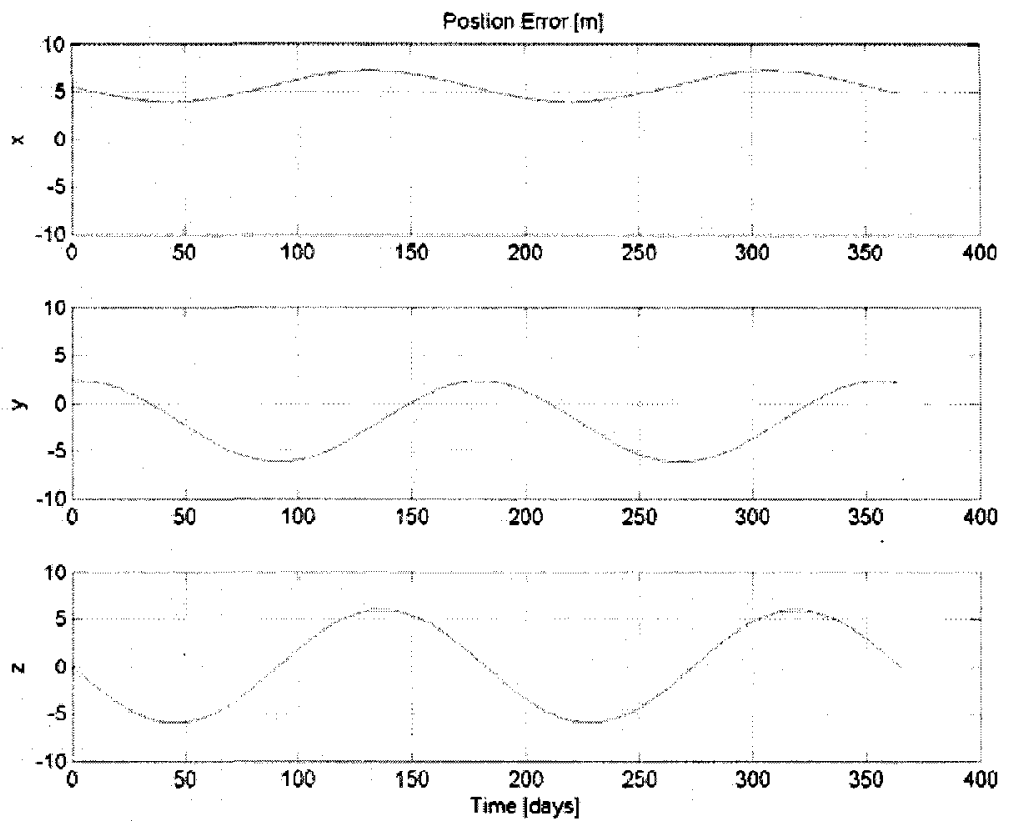

Figure 7.23 - LQR position errors for 1 year (differing initial conditions) 

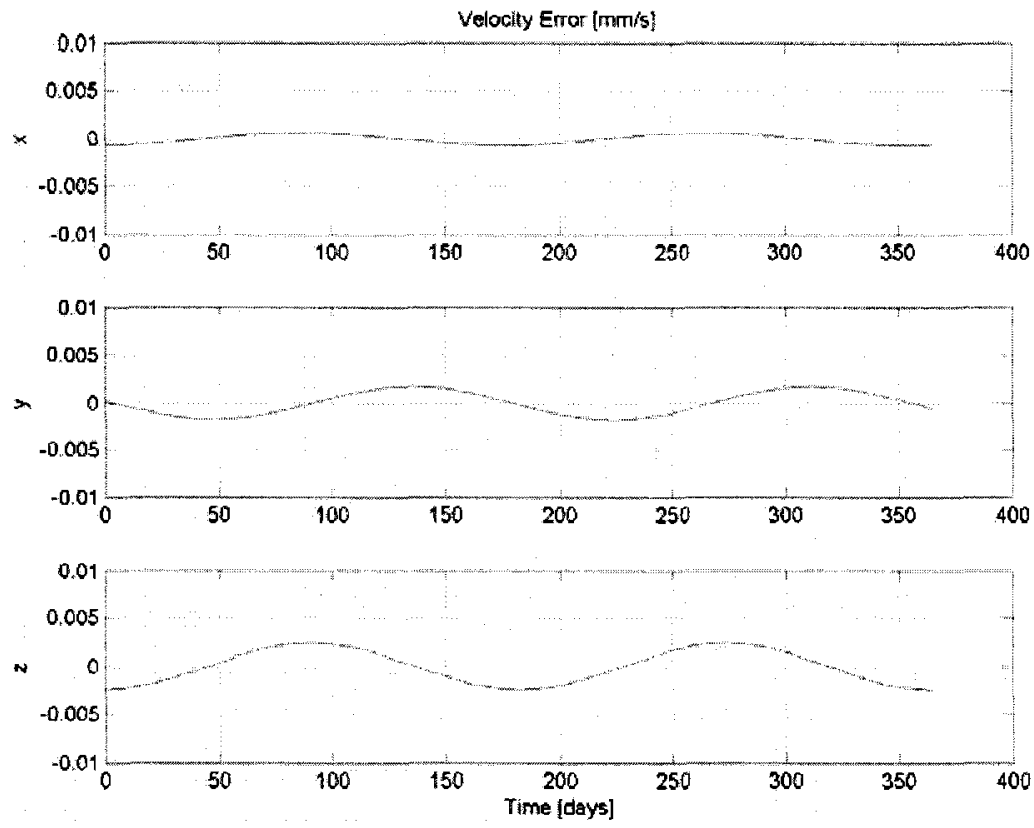

Figure 7.24 - LQR velocity errors for 1 year (differing initial conditions)

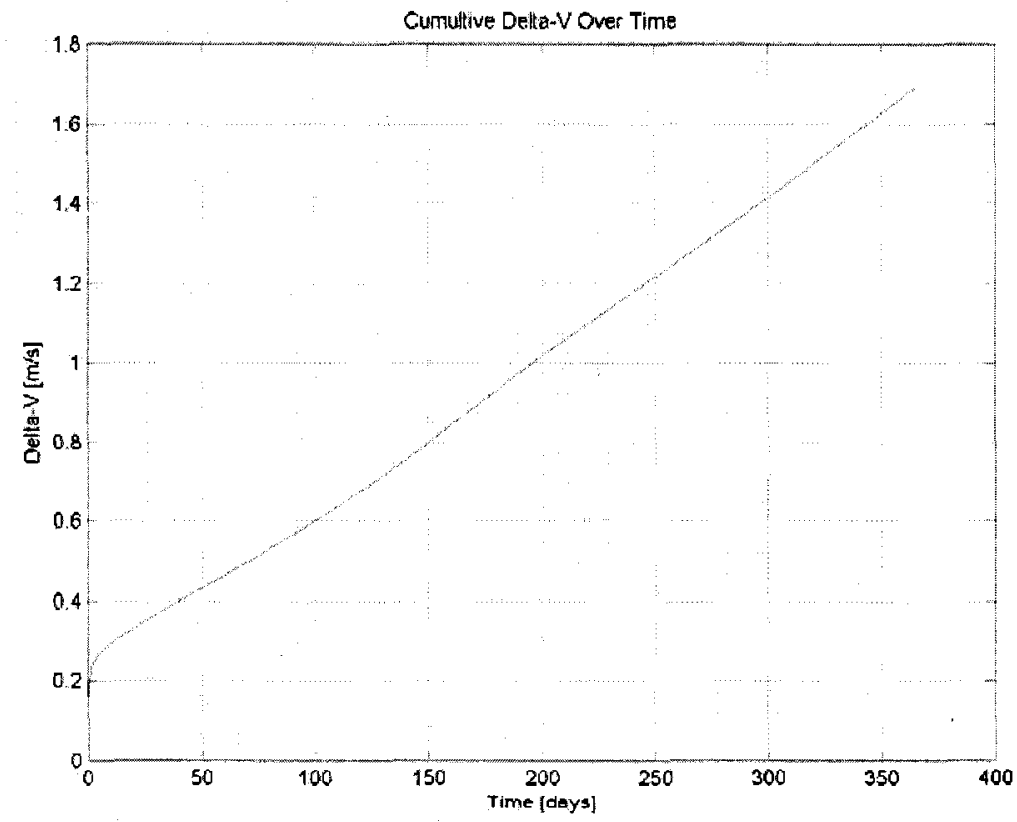

Figure 7.25 - LQR $\Delta v$ profile for 1 year (differing initial conditions) 


\begin{tabular}{|c|c|c|c|c|}
\hline & \multicolumn{3}{|c|}{ Maximum Errors } & \multirow{2}{*}{$\begin{array}{l}4 \times 5 \\
3451\end{array}$} \\
\hline & $x$ & $y$ & 2 & \\
\hline postion $\mathrm{m}$ & 7.2 & 6.1 & 5.9 & \multirow{2}{*}{1.51} \\
\hline velocity [pm/s] & 0.069 & 1.8 & 2.4 & \\
\hline
\end{tabular}

Table 7.8 - LQR results for 1 year (differing initial conditions)

The LQR controlled system does well in maintaining errors within the mission allowance but allows the $\Delta \mathrm{v}$ to increase slightly. The control compensates for the large initial offset immediately, expending a larger amount fuel in the early stage of the mission. Once the error is reduced, the $\Delta v$ returns to a minimum. This phenomenon can be seen in Figure 7.25 Although $\Delta v$ increases compared to the unperturbed system, the increase is only by $0.05 \mathrm{~m} / \mathrm{s}$, concluding that $L Q R$ reacts well under differing initial conditions.

\subsection{Worst Case Scenario Results}

Results pertaining to the LQR controller applied to the nonlinear system with the presence of all modeled disturbances and perturbations are shown in Figures 7.26 through 7.28. Numerical results are listed in Table 7.9. 

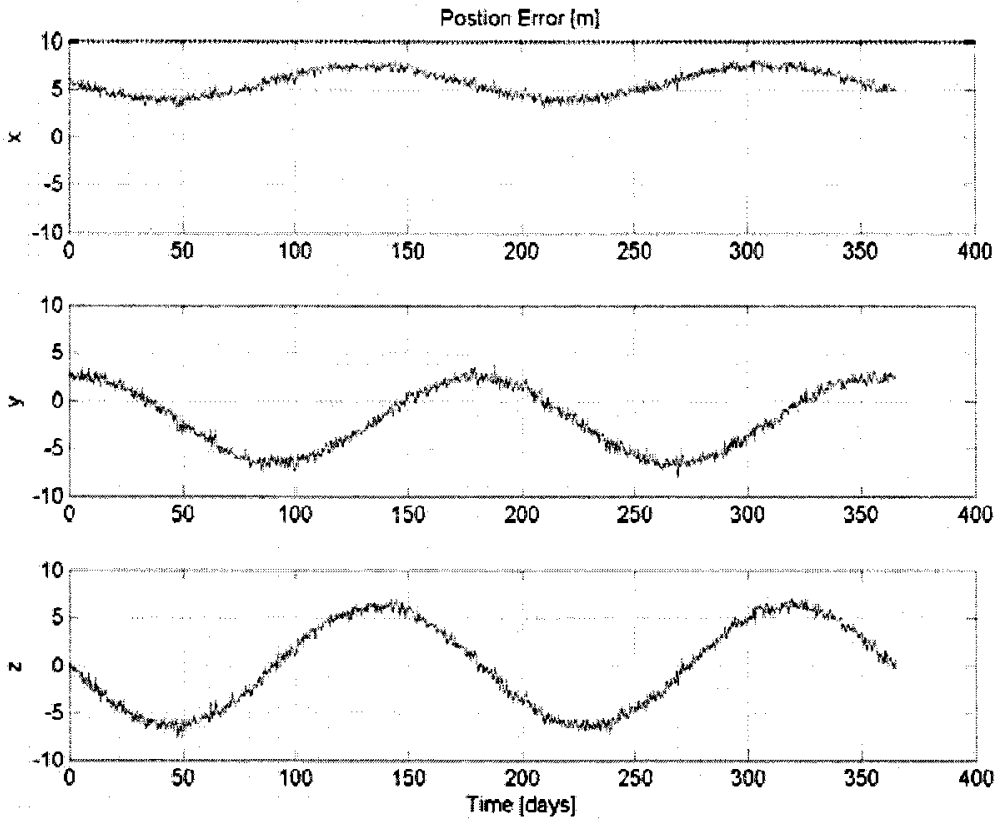

Figure 7.26 - LQR position errors for 1 year (worst case)
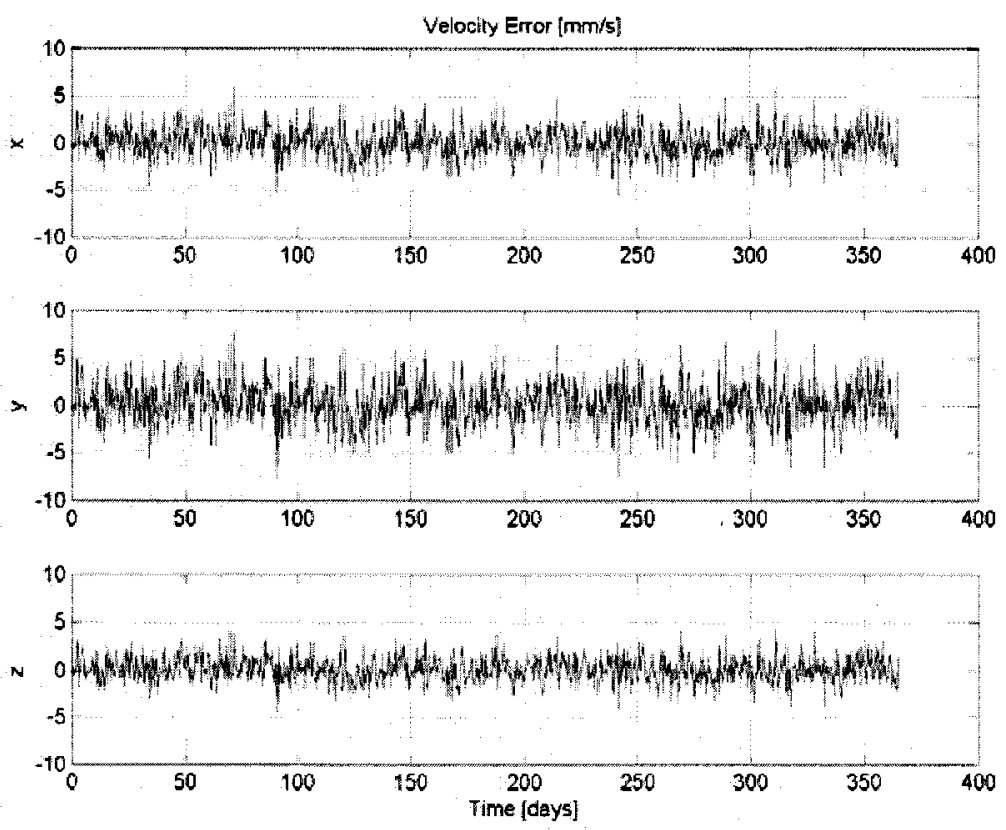

Figure 7.27 - LQR velocity errors for 1 year (worst case) 


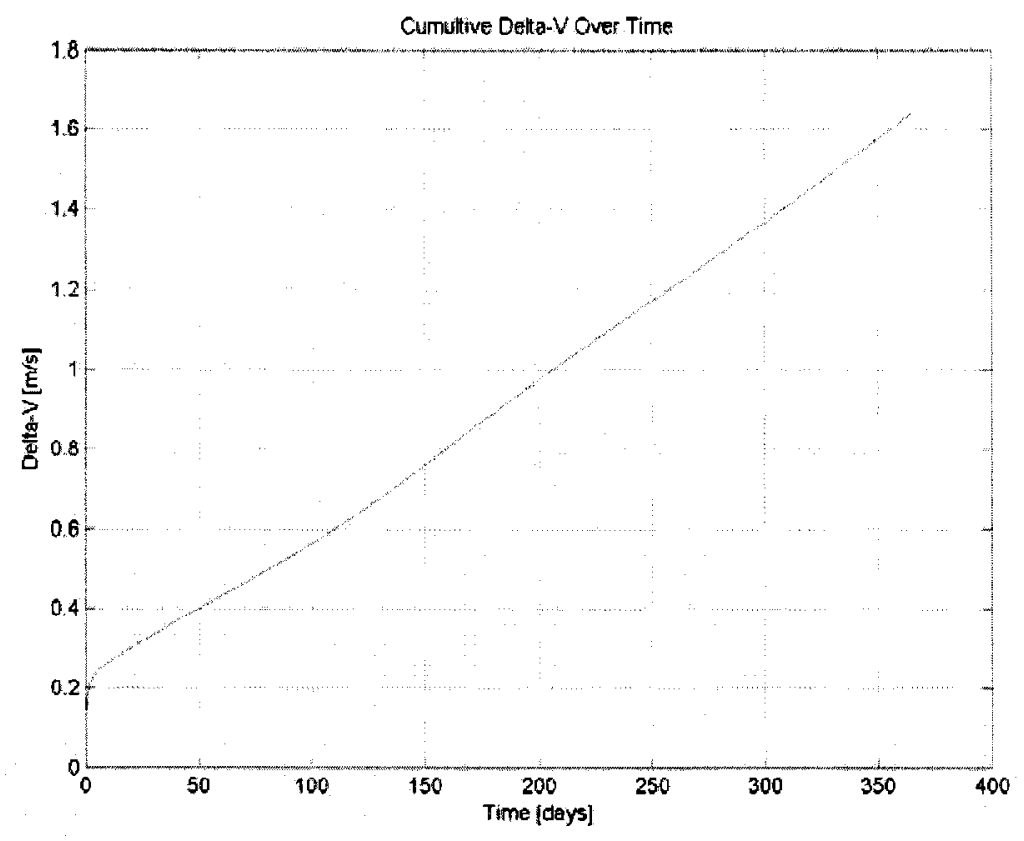

Figure 7.28 - LQR $\Delta v$ profile for 1 year (worst case)

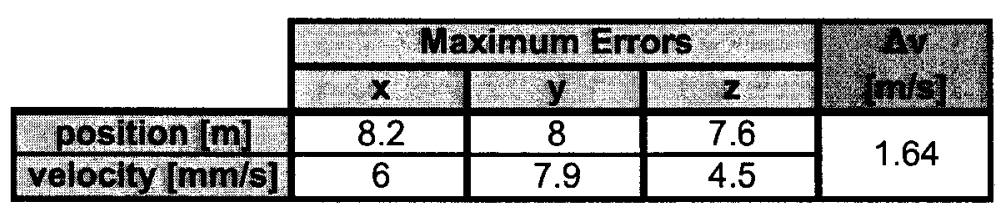

Table 7.9 - LQR results for 1 year (solar radiation pressure)

The results of the worst case scenario experience characteristics similar to the two cases with abnormal behavior, measurement noise and differing initial conditions. However, even in the presence of all the disturbances and perturbations, the position errors are maintained within the accepted allowable tolerances and $\Delta \mathrm{v}$, although slightly greater than the minimum, is preserved at a small value. The results yielded conclude that LQR control is robust enough to maintain stability in the worst case scenario. 


\subsection{Summary}

\begin{tabular}{|c|c|c|c|c|}
\hline & \multicolumn{3}{|c|}{ Max Position Errors $[\mathrm{m}]$} & \multirow{2}{*}{ Mo } \\
\hline & $x$ & $y$ & 2 & \\
\hline Unperturbed & 7.2 & 6.1 & 5.9 & 1.46 \\
\hline Thr. Misalign. & $\overline{7.7}$ & $\overline{6.1}$ & $\overline{5.9}$ & $\overline{1.46}$ \\
\hline Thr. Bias & 7.6 & 6.4 & 6.2 & 1.46 \\
\hline Selferavity & 7.2 & 6.1 & 5.9 & 1.46 \\
\hline Jupiter Gravity & 7.2 & 6.1 & 5.9 & 1.46 \\
\hline SRP & 7.1 & 6.1 & 5.9 & 1.43 \\
\hline Meas. Moise & 8 & 7.9 & $\overline{7.2}$ & 1.46 \\
\hline Differing $1 \mathrm{CS}_{\mathrm{s}}$ & $\overline{7.2}$ & 6.1 & 5.9 & 1.51 \\
\hline Worst Case & 8.2 & 8 & 7.6 & 1.64 \\
\hline
\end{tabular}

Table 7.10 - LQR results (summary)

Reviewing Table 7.10, LQR can be labeled a robust controller. The controller performs well for each case investigated, producing errors within the tolerance and minimizing $\Delta \mathrm{v}$. Although the worst case scenario yields a $\Delta \mathrm{v}$ slightly higher than the unperturbed case, this is expected in the presence of all disturbances and perturbations. LQR can be concluded to be robust for all cases investigated, for the given formation flying mission. 


\section{CHAPTER 8}

\section{H-INFINITY}

\section{$\underline{\text { 8.1 Fundamental Equations }}$}

$\mathrm{H}_{\infty}$ is another form of linear optimal control which takes advantage of the Riccati equation. As explained in section 5.2.3, $\mathrm{H}_{\infty}$ is designed with a two point boundary problem to be robust against disturbances. The equations of this section closely follow the analysis in [5].

\subsubsection{Cost Function}

Consider the cost function associated with the $\mathrm{H}_{\infty}$ controller:

$$
J(\mathbf{K}, w, t, \Delta)=\int_{0}^{t}\left(\widetilde{\mathbf{x}}^{T} \widetilde{\mathbf{x}}-\gamma^{2} w^{T} w\right) d \tau+\widetilde{\mathbf{x}}(t)^{T} \Delta \widetilde{\mathbf{x}}(t)
$$

where $\mathbf{K}$ is the control gain, $w$ is the disturbance, $\Delta$ is a nonnegative definite matrix and $\gamma$ is a design variable. The control law is defined as

$$
\mathbf{u}=\mathbf{K} \widetilde{\mathbf{x}}
$$

There exists a "best" controller, $\mathbf{K}^{*}$, and a "worst" disturbance, $w^{*}$ such that

$$
J\left(\mathbf{K}^{*}, w, t, \Delta\right) \leq J\left(\mathbf{K}^{*}, w^{*}, t, \Delta\right) \leq J\left(\mathbf{K}, w^{*}, t, \Delta\right)
$$

The controller $\mathbf{K}^{*}$ provides a minimum $J$ for any disturbance, $w$. 


\subsubsection{Minimization Problem}

The CR3BP linearized equations of motion defined in state-space with additional disturbance and input terms are

$$
\begin{aligned}
& \dot{\mathbf{x}}=\mathbf{A} \mathbf{x}+\mathbf{B}_{1} w+\mathbf{B}_{2} \mathbf{u} \\
& \mathbf{y}=\mathbf{C} \mathbf{x}
\end{aligned}
$$

To approach the minimization problem the disturbance, $w$, is set to $w^{*}$ to find the best input, $\mathbf{u}^{*}$. Following [5], the ideal input is found to be

$$
\mathbf{u}^{*}=-\mathbf{B}_{1}{ }^{T} \lambda, \quad 0 \leq t \leq T
$$

where

$$
\lambda(t)=\int_{i}^{T} \Phi^{T}(\tau, t) C^{T} C \mathbf{x}^{*} d \tau+\Phi(T, t) \Delta \mathbf{x}^{*}(T)
$$

and $\Phi$ is the transition matrix corresponding to $\mathbf{A}$ and

$$
\dot{\mathbf{x}}^{*}=\mathbf{A} \mathbf{x}^{*}+\mathbf{B}_{1} w^{*}+\mathbf{B}_{2} \mathbf{u}^{*}
$$

\subsubsection{Maximization Problem}

Approaching the maximization problem, $w^{*}$ is found by setting $\mathbf{u}$ equal to $\mathbf{u}^{*}$. Once again following [5],

$$
w^{*}=\gamma^{-2} \mathbf{B}_{1} \lambda
$$

\subsubsection{Two-Point-Boundary Problem}

Joining the minimization and maximization problem forms the two-point boundary problem, which can be used to find a solution for $\lambda$. Differentiating Equation (8.6) with respect to time,

$$
\dot{\lambda}(t)=-\mathbf{A}^{T} \lambda-C^{T} C \mathbf{x}^{*}
$$


Combining Equations (8.5), (8.7) and (8.9),

$$
\left[\begin{array}{c}
\dot{\mathbf{x}}^{*} \\
\dot{\lambda}
\end{array}\right]=\left[\begin{array}{cc}
\mathbf{A} & -\left(\mathbf{B}_{1} \mathbf{B}_{2}{ }^{T}-\gamma^{-2} \mathbf{B}_{1} \mathbf{B}_{2}\right) \\
-\mathbf{C}^{T} \mathbf{C} & -\mathbf{A}^{T}
\end{array}\right]\left[\begin{array}{c}
\mathbf{x}^{*} \\
\lambda
\end{array}\right]
$$

with boundary conditions

$$
\left[\begin{array}{c}
\mathbf{x}^{*}(0) \\
\lambda(T)
\end{array}\right]=\left[\begin{array}{c}
\mathbf{x}_{0}^{*} \\
\Delta \mathbf{x}^{*}(T)
\end{array}\right]
$$

If $\Phi(t, T)$ becomes the transition matrix for Equation (8.10) such that

$$
\frac{d}{d t} \Phi(t, T)=\mathbf{H} \Phi(t, T), \quad \Phi(T, T)=\mathbf{I}
$$

where

$$
\mathbf{H}=\left[\begin{array}{cc}
\mathbf{A} & -\left(\mathbf{B}_{1} \mathbf{B}_{2}{ }^{T}-\gamma^{-2} \mathbf{B}_{1} \mathbf{B}_{2}\right) \\
-\mathbf{C}^{T} \mathbf{C} & -\mathbf{A}^{T}
\end{array}\right]
$$

then $(8.10)$ can be written as

$$
\left[\begin{array}{c}
\mathbf{x}^{*}(t) \\
\lambda(t)
\end{array}\right]=\left[\begin{array}{ll}
\Phi_{11}(t, T) & \Phi_{12}(t, T) \\
\Phi_{21}(t, T) & \Phi_{22}(t, T)
\end{array}\right]\left[\begin{array}{c}
\mathbf{x}^{*}(t) \\
\lambda(t)
\end{array}\right]
$$

Applying the second boundary condition in Equation (8.11) to Equation (8.14) yields

$$
\lambda(t)=\mathbf{P x}^{*}(t)
$$

where

$$
\mathbf{P}(t)=\left(\Phi_{21}(t, T)+\Phi_{22}(t, T) \Delta\right)\left(\Phi_{11}(t, T)+\Phi_{21}(t, T) \Delta\right)^{-1}
$$

which is also a solution to the steady-state Riccati equation

$$
\mathbf{A}^{T} \mathbf{P}+\mathbf{P A}-\mathbf{P}\left(\mathbf{B}_{2} \mathbf{B}_{2}{ }^{T}-\gamma^{-2} \mathbf{B}_{1} \mathbf{B}_{1}{ }^{T}\right) \mathbf{P}+\mathbf{C}^{T} \mathbf{C}=0
$$

Thus Equations (8.5) and (8.7) become

$$
\mathbf{u}^{*}=-\mathbf{B}_{2} \mathbf{P} \widetilde{\mathbf{x}}
$$




$$
w^{*}=\gamma^{-2} \mathbf{B}_{1}{ }^{T} \mathbf{P} \widetilde{\mathbf{x}}
$$

and the control gain becomes

$$
\mathbf{K}=-\mathbf{B}_{2} \mathbf{P}
$$

\subsubsection{Control Law with Disturbance Contribution}

The control law in (8.18) only has a relation to the state error. However it is desired for $\mathrm{H}_{\infty}$ to also base the control on the disturbance.

Consider the control law

$$
\mathbf{u}-\mathbf{u}^{*}=\mathbf{V}(\mathbf{x}-\hat{\mathbf{x}})
$$

where $\hat{\mathbf{x}}$ is the duplicate state defined by

$$
\dot{\hat{\mathbf{x}}}=\mathbf{A} \hat{\mathbf{x}}+\mathbf{B}_{1} w^{*}+\mathbf{B}_{2} \mathbf{u}
$$

and $\mathbf{V}$ is a weighting matrix. The duplicate state is an estimation of the actual state if the worst error were present. This produces an estimation of the disturbance which is incorporated into the control law.

Substituting Equation (8.18) into (8.21), the new control law becomes

$$
\mathbf{u}=-\left(\mathbf{B}_{2}{ }^{T} \mathbf{P}+\mathbf{V}\right) \mathbf{x}-\mathbf{V} \hat{\mathbf{x}}
$$

The block diagram can be seen in Figure 8.1. 


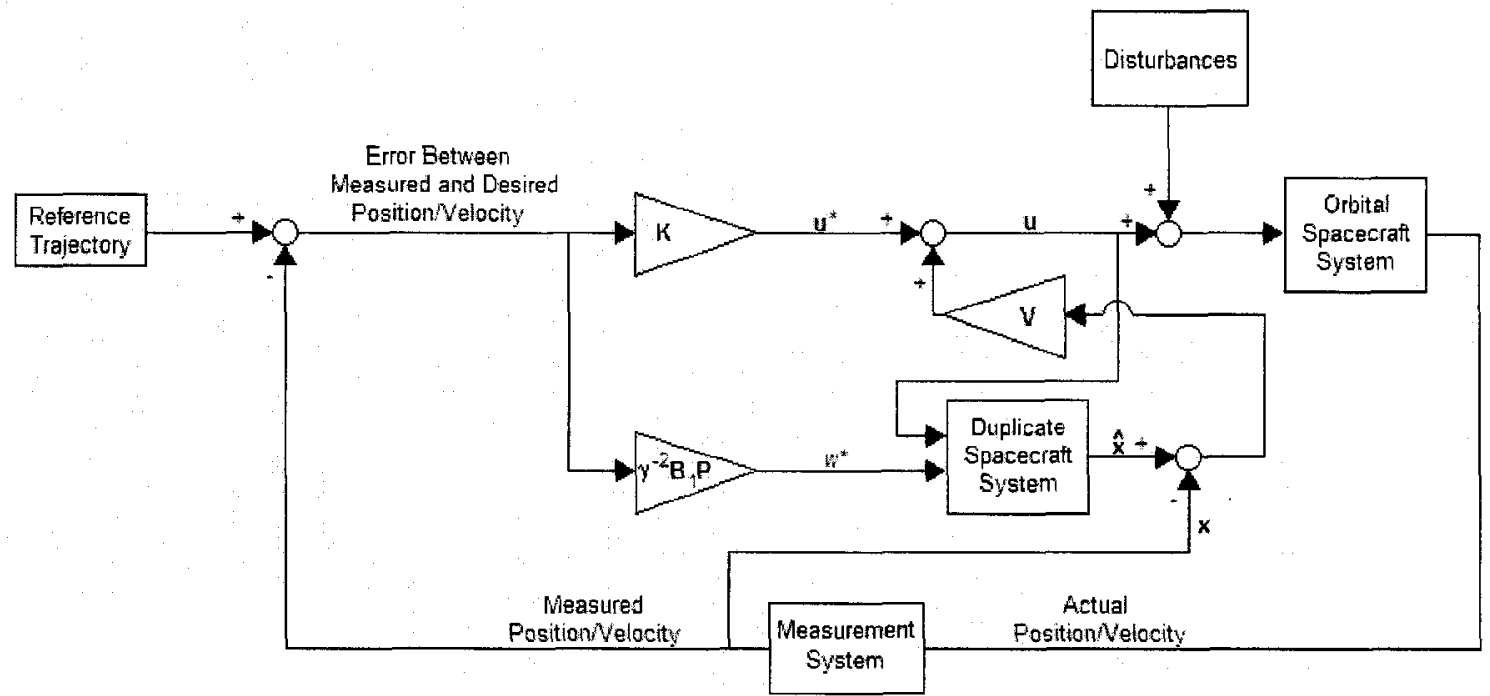

Figure 8.1 - $\mathbf{H}_{\infty}$ block diagram

\subsection{Gain Selection}

Unlike the previous two control designs of PID and LQR, the gains for $\mathrm{H}_{\infty}$ are chosen by first selecting a set of desired poles for the linearized equations of motion. Using knowledge of the closed-loop poles and their relation to a transient response, poles are chosen. The poles are implemented in the nonlinear CR3BP system then tuned appropriately to obtain the following numbers:

$$
\lambda=\left[\begin{array}{l}
-5 \cdot 10^{-3} \pm 7 \cdot 10^{-5} i \\
-7 \cdot 10^{-3} \pm 6 \cdot 10^{-6} i \\
-2 \cdot 10^{-3} \pm 7 \cdot 10^{-4} i
\end{array}\right]
$$

and setting $\gamma=2$, the resulting steady-state Riccati solution is 


$$
\mathbf{P}=\left[\begin{array}{cccccc}
0 & 0 & 0 & 0 & 0 & 0 \\
0 & 0 & 0 & 0 & 0 & 0 \\
0 & 0 & 0 & 0 & 0 & 0 \\
2.8 \cdot 10^{-5} & -1.6 \cdot 10^{-5} & 3.3 \cdot 10^{-6} & 1.2 \cdot 10^{-2} & -3.2 \cdot 10^{-3} & 4.3 \cdot 10^{-4} \\
-1.1 \cdot 10^{-5} & 2.8 \cdot 10^{-5} & -1.4 \cdot 10^{-5} & -2.7 \cdot 10^{-3} & 1.2 \cdot 10^{-3} & -2 \cdot 10^{-3} \\
9 \cdot 10^{-6} & -6.2 \cdot 10^{-6} & 2.2 \cdot 10^{-5} & 9 \cdot 10^{-4} & -1.1 \cdot 10^{-3} & 1.3 \cdot 10^{-2}
\end{array}\right]
$$

The weighting matrix, $\mathbf{V}$, is also set to $\mathbf{V}=\left[\mathbf{I}_{3}, \mathbf{I}_{3}\right] \cdot 10^{-7}$.

\subsection{Unperturbed System Results}

Results pertaining to the $\mathrm{H}_{\infty}$ controller applied to the nonlinear system without disturbances or perturbations are shown in Figures 8.2 through 8.4.
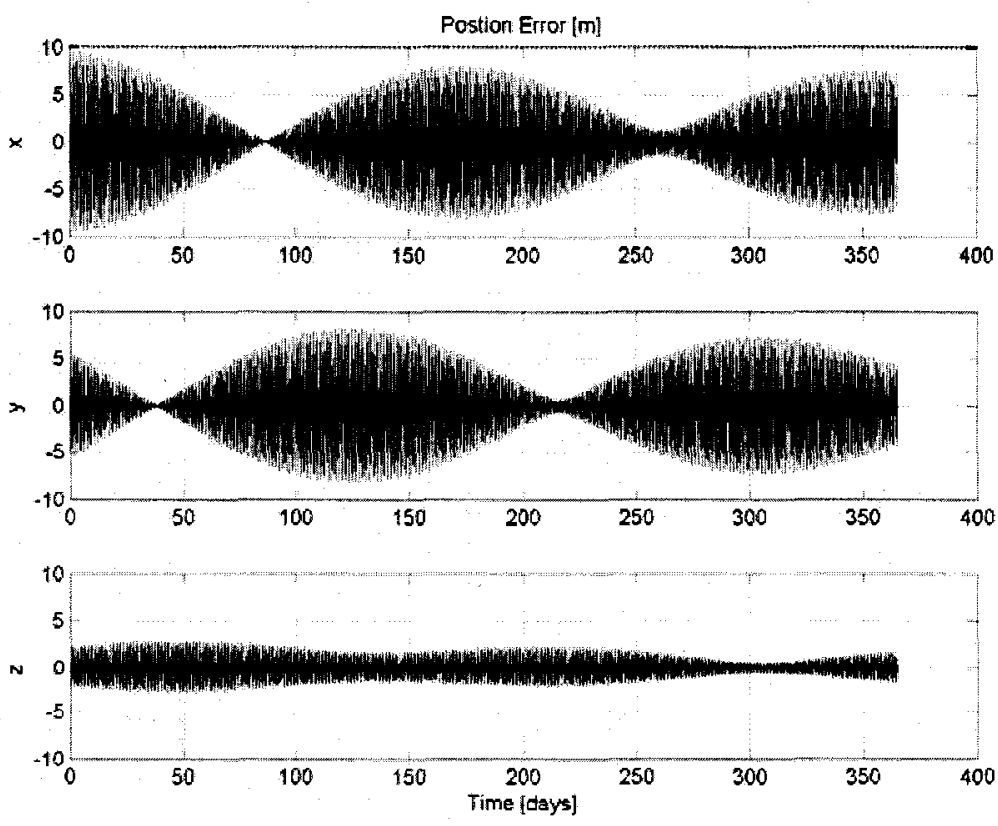

Figure 8.2 - $H_{\infty}$ position errors for 1 year (unperturbed) 

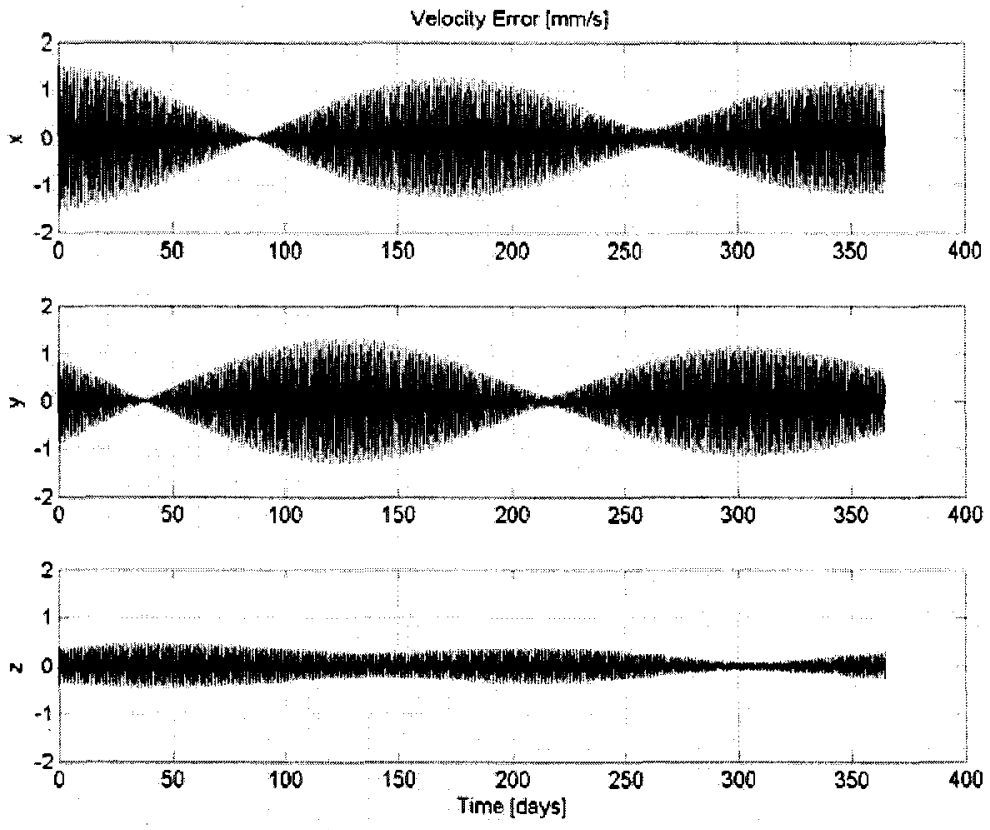

Figure $8.3-H_{\infty}$ velocity errors for 1 year (unperturbed)

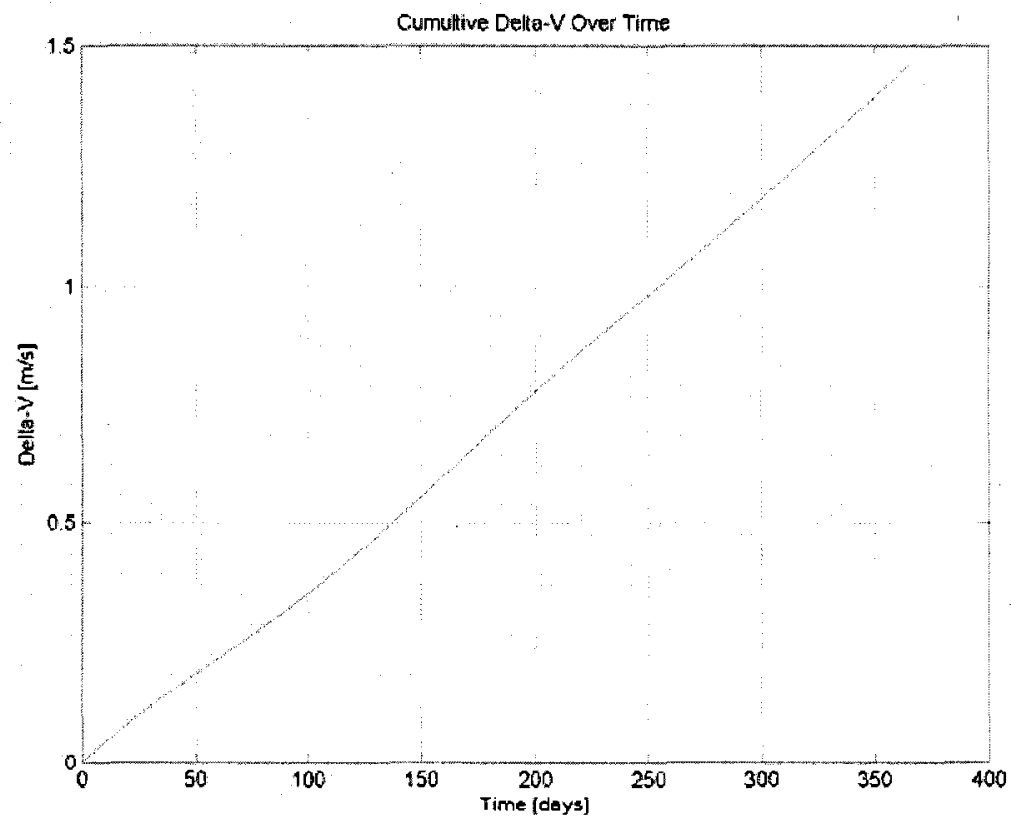

Figure 8.4 - $\mathrm{H}_{\infty} \Delta v$ profile for 1 year (unperturbed) 


\begin{tabular}{|c|c|c|c|c|}
\cline { 2 - 5 } \multicolumn{1}{c|}{} & \multicolumn{3}{c|}{ Maximum Errors } \\
\cline { 2 - 5 } \multicolumn{1}{c|}{} & $\mathbf{x}$ & $\mathbf{y}$ & $\mathbf{z}$ & \\
\hline position $[\mathbf{m}]$ & 9.3 & 8.2 & 2.9 & 1.46 \\
\hline velocity $[\mathbf{m m} / \mathrm{s}]$ & 1.4 & 1.3 & 0.47 & \\
\hline
\end{tabular}

Table $8.1-\mathbf{H}_{\infty}$ results for 1 year (unperturbed)

Table 8.1 lists the $\Delta v$ and maximum errors for position and velocity once the orbit is stabilized. The controller performs well, maintaining errors within the allowable tolerances. However, a great deal of oscillations occur in both the position and velocity errors. The oscillation appear to be a modulation of two frequencies. The lower frequency corresponds to the natural oscillation of the spacecraft's state. That is the $\mathrm{x}$ error peaks at the maximum $\mathrm{x}$-coordinate value in orbit. This frequency can be seen in the error responses of the previous two controllers. The secondary, higher frequency corresponds to the internal frequency of the controller. Within the controller is a secondary duplicate system that produces an estimate of the state with a much larger disturbance than what is present. This larger estimate causes a larger overshoot which continually is present. Oscillations are not desirable in a spacecraft, since it causes wear and tear, especially at constant frequencies. Even though oscillations are present, the errors are maintained in the tolerance while concurrently minimizing $\Delta \mathrm{v}$. 


\subsection{Thruster Misalignment Results}

Results pertaining to the $\mathrm{H}_{\infty}$ controller applied to the nonlinear system with thruster misalignments of a tenth of a degree are shown in Figures 8.5 through 8.8. Numerical results are listed in Table 8.2.
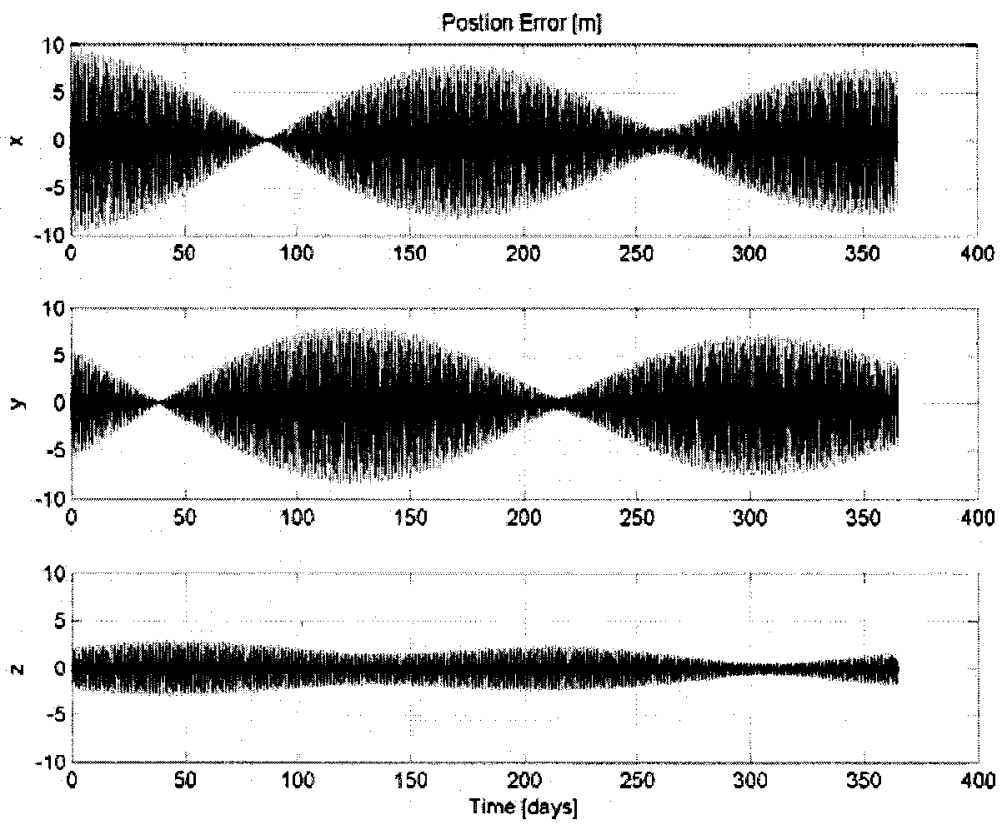

Figure 8.5 - $\mathbf{H}_{\infty}$ position errors for 1 year (thruster misalignments) 

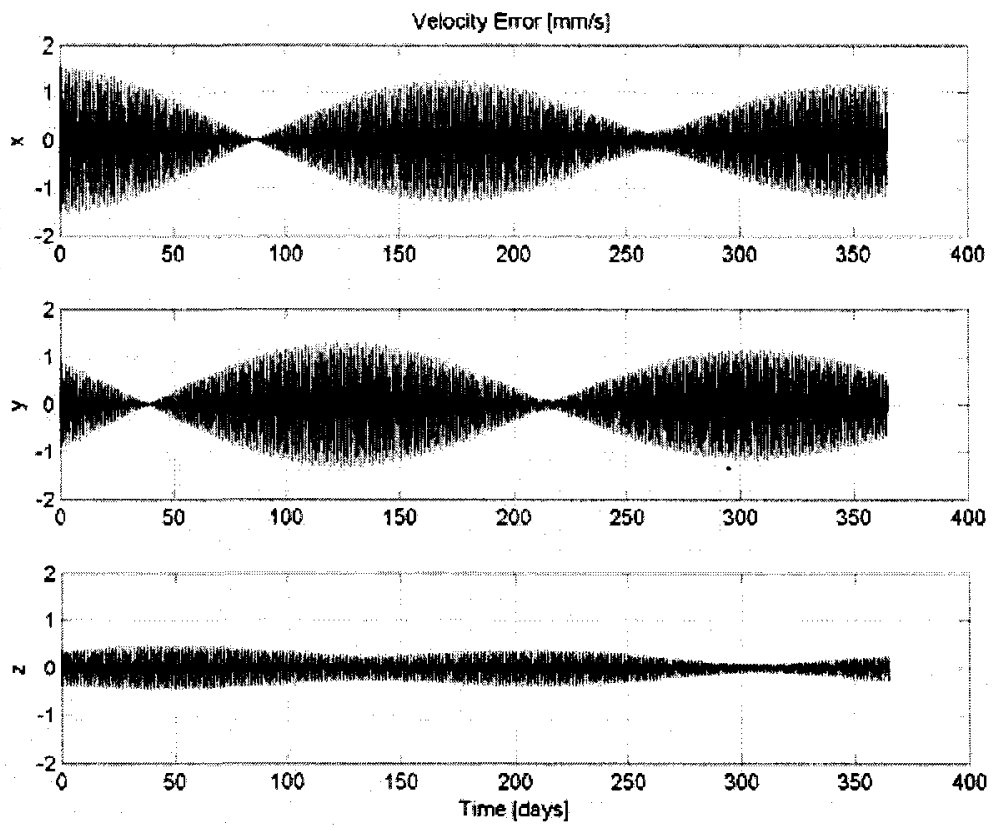

Figure 8.6 - $\mathbf{H}_{\infty}$ velocity errors for 1 year (thruster misalignments)

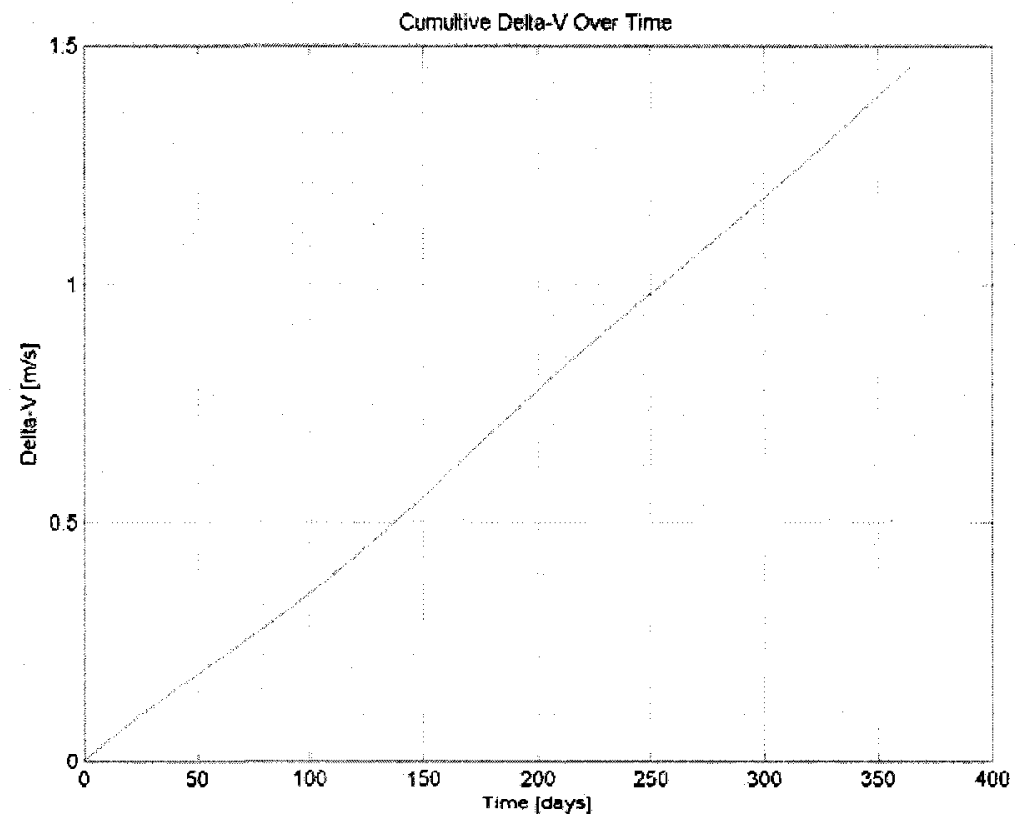

Figure $8.7-H_{\infty} \Delta v$ profile for 1 year (thruster misalignments) 


\begin{tabular}{|c|c|c|c|c|}
\hline & \multicolumn{3}{|c|}{ Maximum Errors } & \multirow{2}{*}{ Pry } \\
\hline & $x$ & thy & 2 & \\
\hline position [m] & 9.4 & 8.4 & 2.9 & \multirow{2}{*}{1.46} \\
\hline velocity $[\mathrm{mm} / \mathrm{s}]$ & 1.5 & 1.3 & 0.46 & \\
\hline
\end{tabular}

Table $8.2-\mathrm{H}_{\infty}$ results for 1 year (thruster misalignments)

The controller performs very similar to that of the unperturbed system, maintaining the same errors and a minimal $\Delta \mathrm{v}$, with slightly increased position and velocity errors. The $\mathrm{H}_{\infty}$ controller is shown to maintain desirable error and fuel results, however, undesirable oscillations are still present.

\subsection{Thruster Bias Results}

Results pertaining to the $\mathrm{H}_{\infty}$ controller applied to the nonlinear system with thruster biases of $-5 \%$ are shown in Figures 8.8 through 8.10. Numerical results are listed in Table 8.3. 

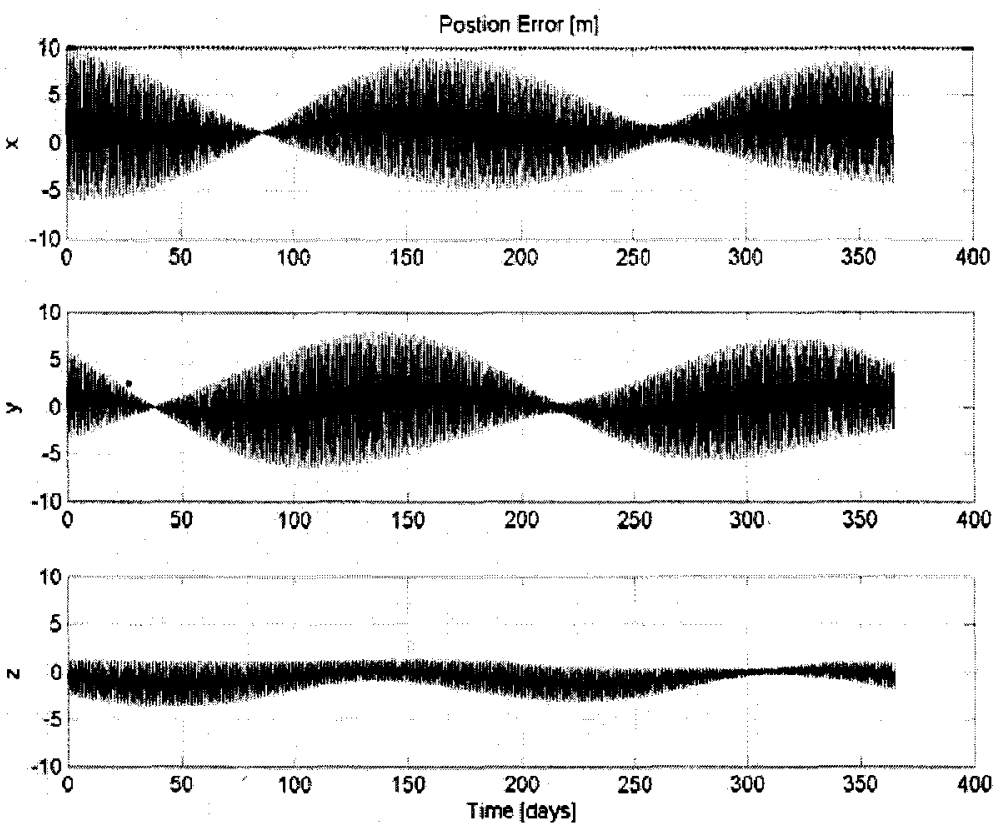

Figure 8.8 - $\mathbf{H}_{\infty}$ position errors for 1 year (thruster bias)
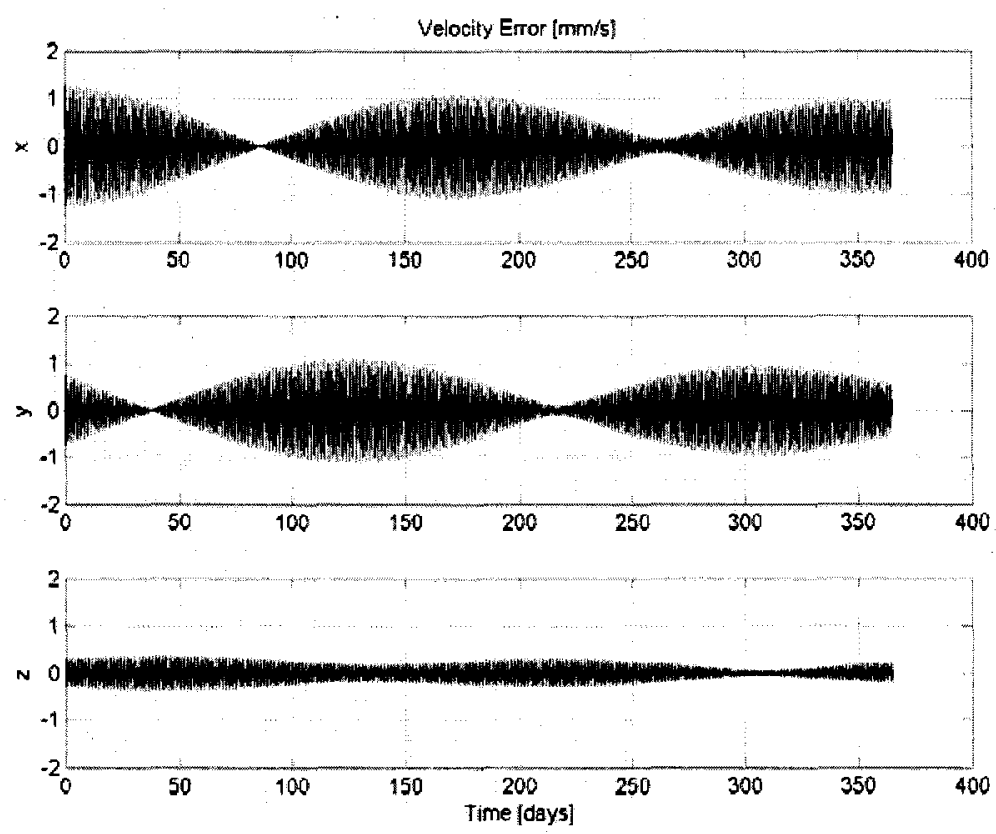

Figure 8.9 - $\mathbf{H}_{\infty}$ velocity errors for 1 year (thruster bias) 


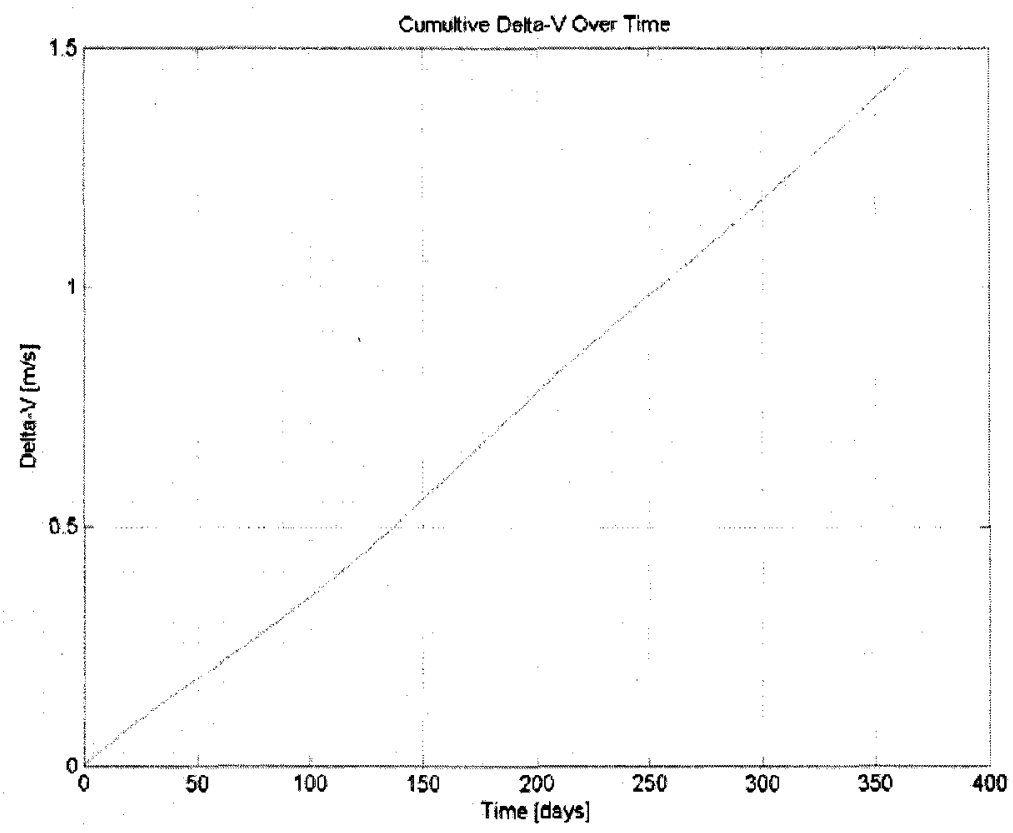

Figure 8.10 - $\mathbf{H}_{\infty} \Delta v$ profile for 1 year (thruster bias)

\begin{tabular}{|c|c|c|c|c|}
\cline { 2 - 5 } \multicolumn{1}{c|}{} & \multicolumn{4}{c|}{ Maximum Errors } \\
\cline { 2 - 5 } \multicolumn{1}{c|}{} & $\mathbf{x}$ & $\mathbf{y}$ & $\mathbf{z}$ & 1 \\
\hline position $[\mathrm{m}]$ & 9.6 & 7.9 & 3.6 & 1.46 \\
\hline velocity $[\mathrm{mm} / \mathrm{s}]$ & 1.2 & 1.1 & 0.39 & \\
\hline
\end{tabular}

Table $8.3-\mathbf{H}_{\infty}$ results for 1 year (thruster bias)

Figures 8.8 and 8.9 show the position and velocity errors, which are similar to the unperturbed results. However, undesirable oscillations are still present yielding the $\mathrm{H}_{\infty}$ controller to not provide acceptable results against thruster bias. 


\subsection{Self-Gravity Results}

Results pertaining to the $\mathrm{H}_{\infty}$ controller applied to the nonlinear system with self-gravity from the leader satellite are shown in Figures 8.11 through 8.13. Numerical results are listed in Table 8.4.
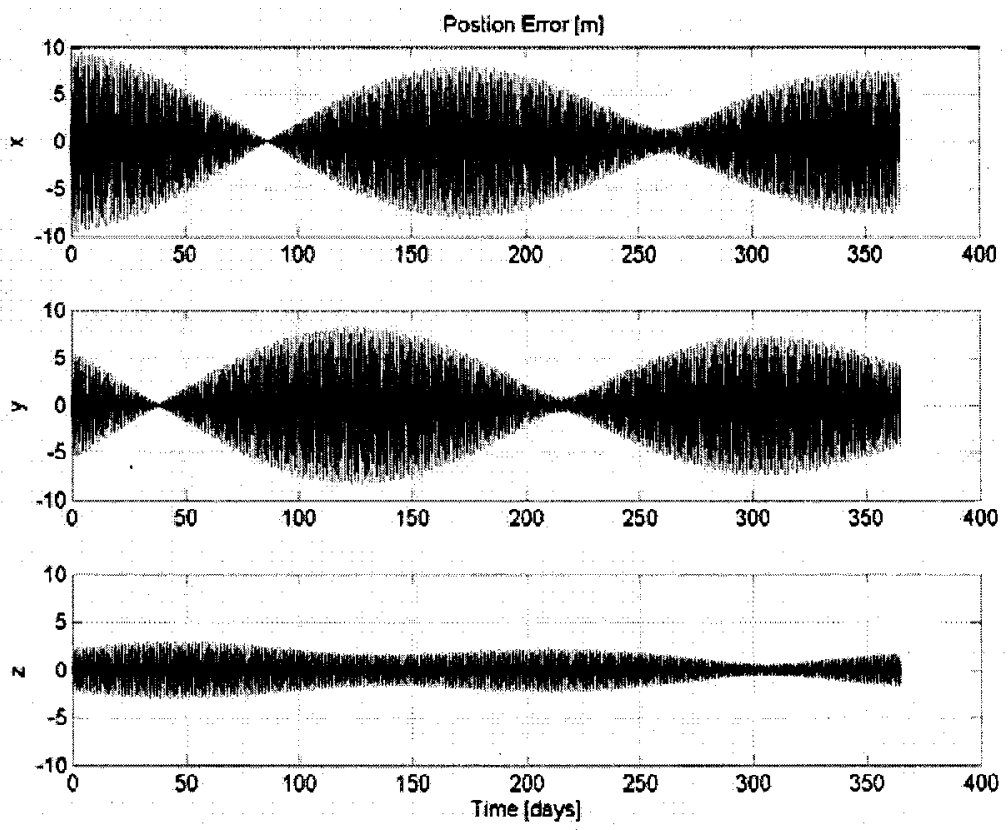

Figure 8.11 - $\mathbf{H}_{\infty}$ position errors for 1 year (self-gravity) 

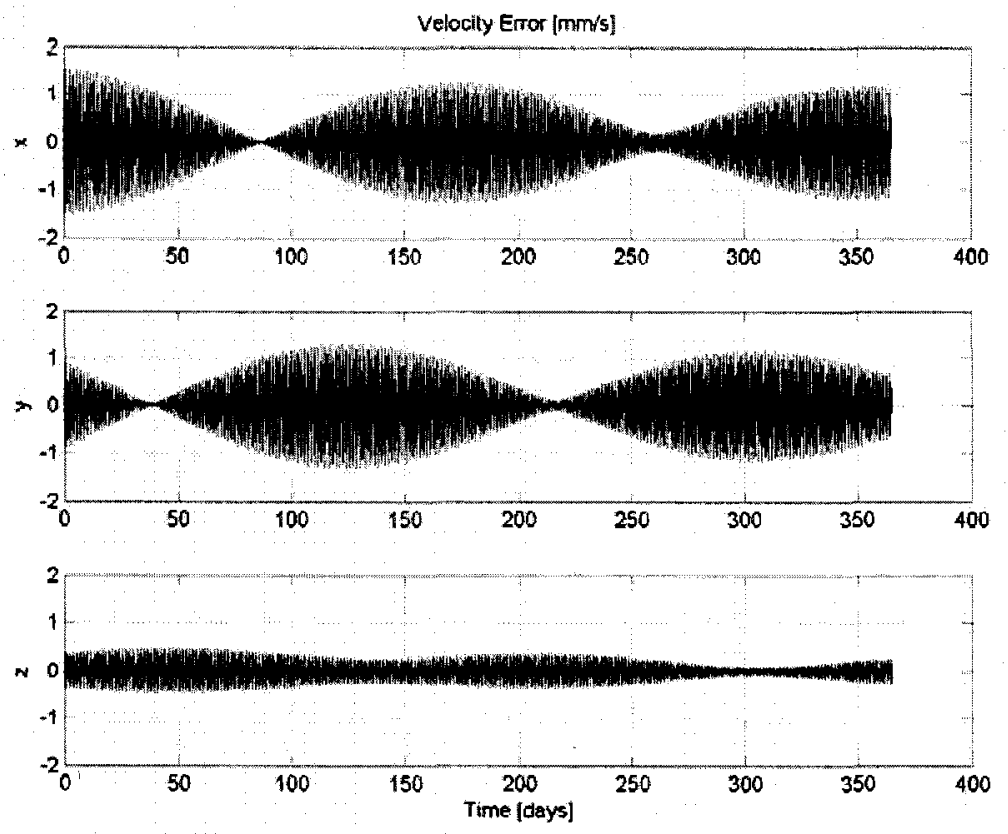

Figure $8.12-\mathbf{H}_{\infty}$ velocity errors for 1 year (self-gravity)

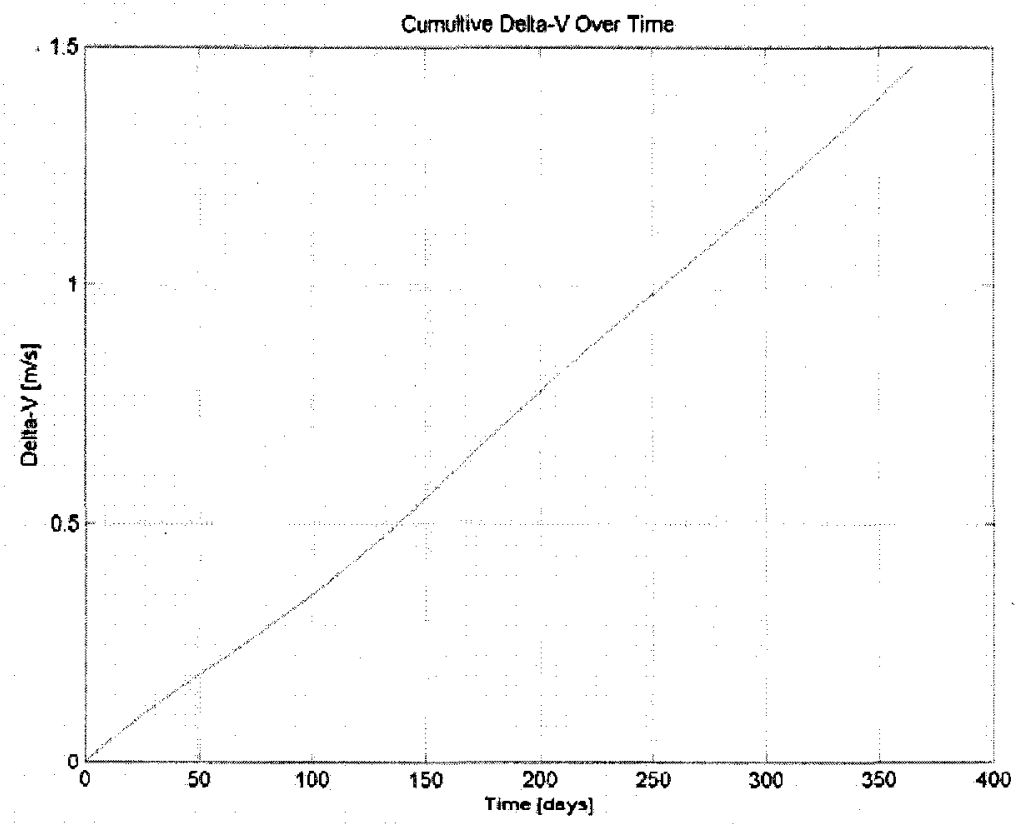

Figure 8.13 - $H_{\infty} \Delta v$ profile for 1 year (self-gravity) 


\begin{tabular}{|c|c|c|c|c|}
\hline & \multicolumn{4}{|c|}{ Waximum Errors } \\
\hline & $x$ & $y$ & Hryes & \\
\hline Dosillon me & 9.3 & 8.2 & 2.9 & 14 \\
\hline velocity [mm/s] & 1.5 & 1.3 & 0.47 & \\
\hline
\end{tabular}

Table $8.4-\mathbf{H}_{\infty}$ results for 1 year (self-gravity)

The controller produces results equal to those of the unperturbed system. The $\mathrm{H}_{\infty}$ controller yields insignificant results against self-gravity but still results in undesirable oscillations.

\subsection{Jupiter Gravity Results}

Results pertaining to the $\mathrm{H}_{\infty}$ controller applied to the nonlinear system while experiencing gravitational forces due to Jupiter are shown in Figures 8.14 through 8.18. Numerical results are listed in Table 8.5. 

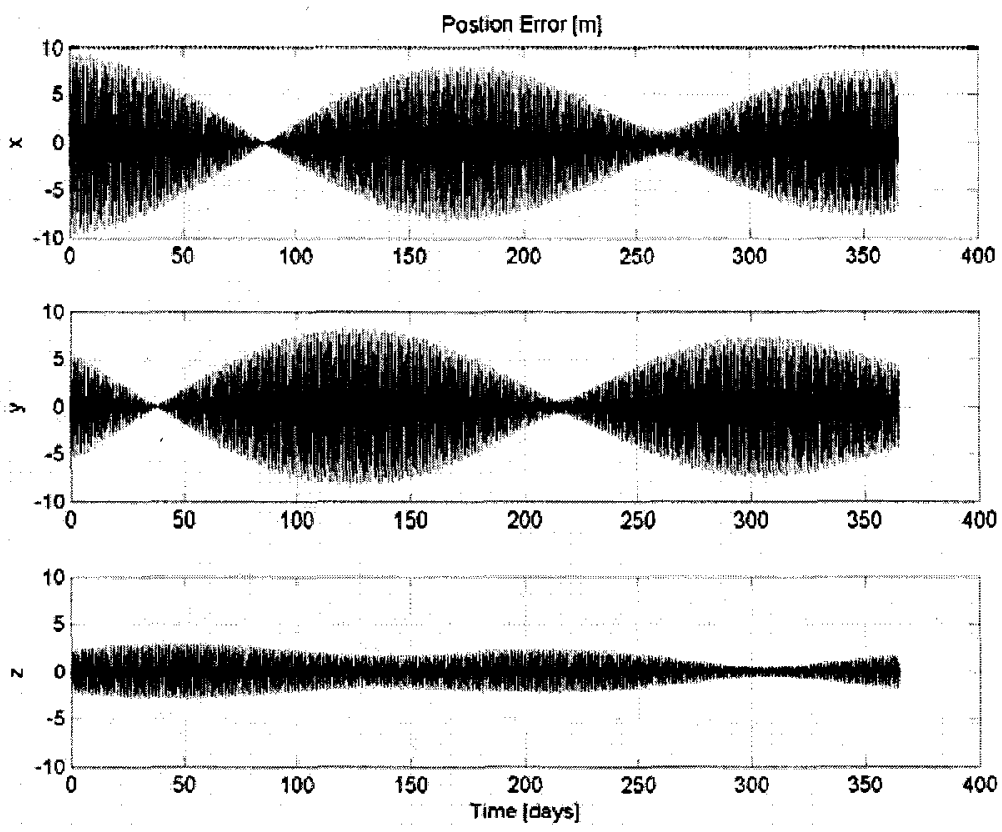

Figure 8.14 - $H_{\infty}$ position errors for 1 year (Jupiter)
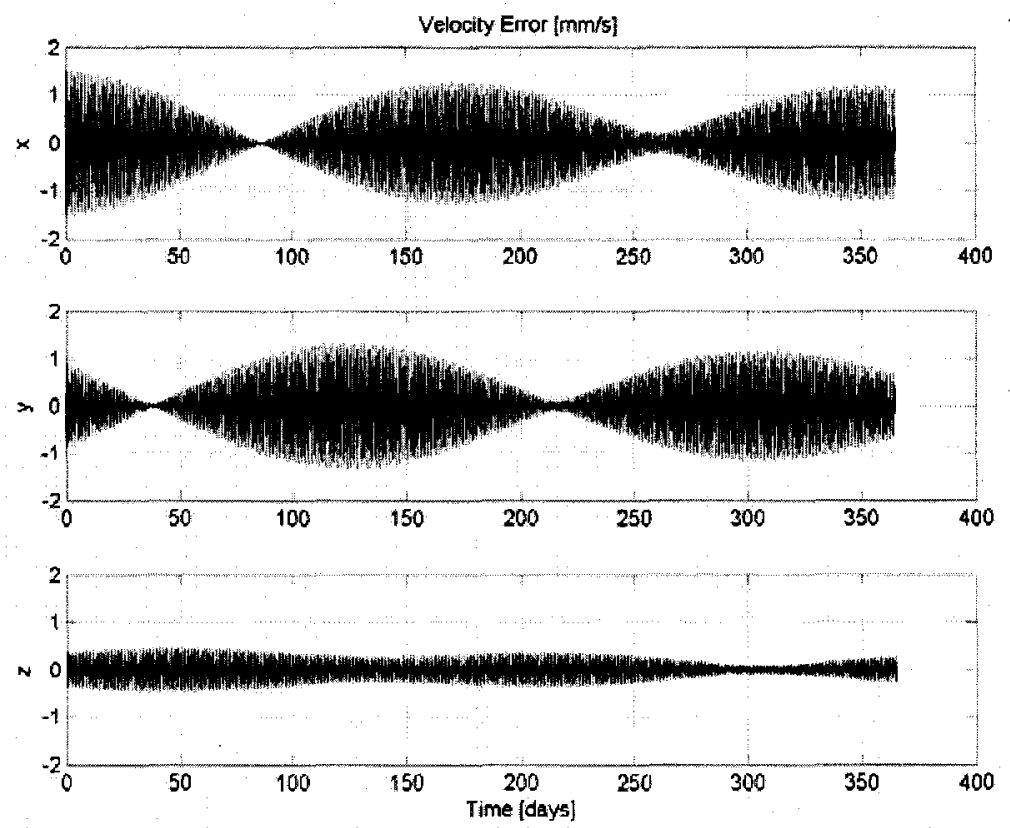

Figure 8.15 - $\mathbf{H}_{\infty}$ velocity errors for 1 year (Jupiter) 


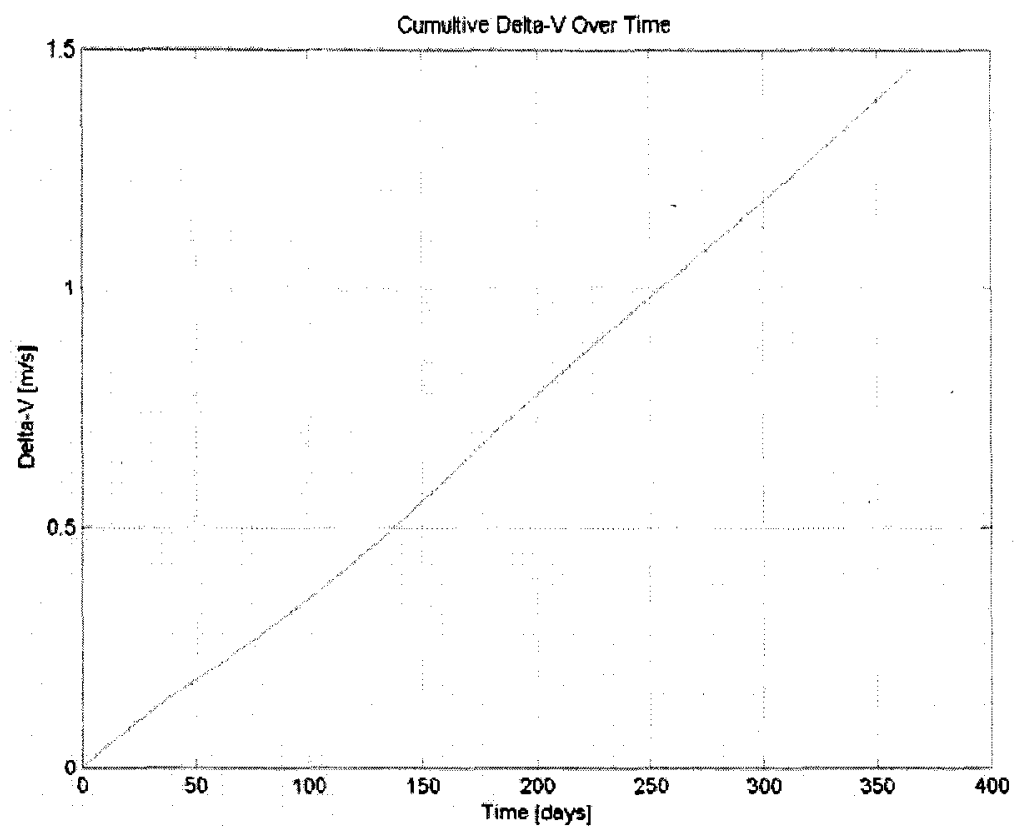

Figure 8.16 - $H_{\infty} \Delta v$ profile for 1 year (Jupiter)

\begin{tabular}{|c|c|c|c|c|}
\hline & \multicolumn{3}{|c|}{ Maximum Erors } & \multirow{2}{*}{ 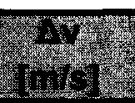 } \\
\hline & $x$ & $y$ & & \\
\hline positio & 9.3 & 8.3 & 2.9 & \multirow{2}{*}{1.46} \\
\hline Velocity $[\mathrm{mm} / \mathrm{s}]$ & 1.5 & 1.3 & 0.47 & \\
\hline
\end{tabular}

Table $8.5-H_{\infty}$ results for 1 year (Jupiter)

Once again, the controller yields results equal to those of the unperturbed system. Unacceptable oscillations are still present, however $H_{\infty}$ yields negligible effects against Jupiter's gravity. 


\subsection{Solar Radiation Pressure Results}

Results pertaining to the $\mathrm{H}_{\infty}$ controller applied to the nonlinear system with the presence of solar radiation pressure are shown in Figures 8.17 through 8.19. Numerical results are listed in Table 8.8.
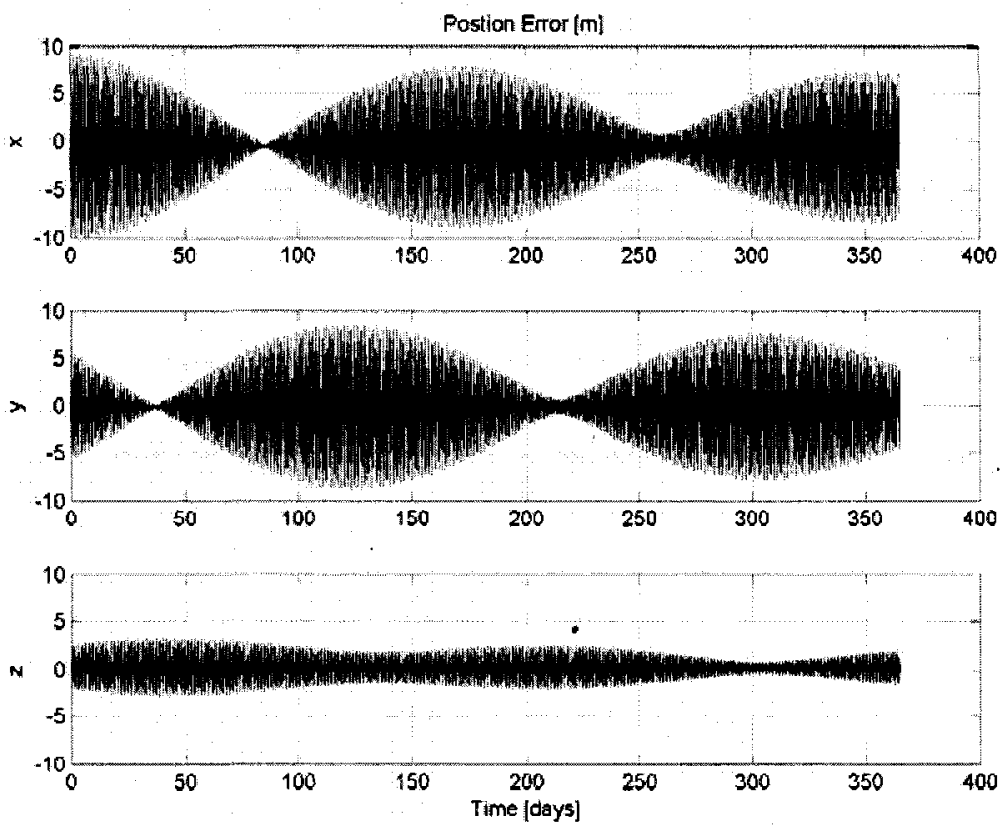

Figure 8.17 - $\mathbf{H}_{\infty}$ position errors for 1 year (solar radiation pressure) 

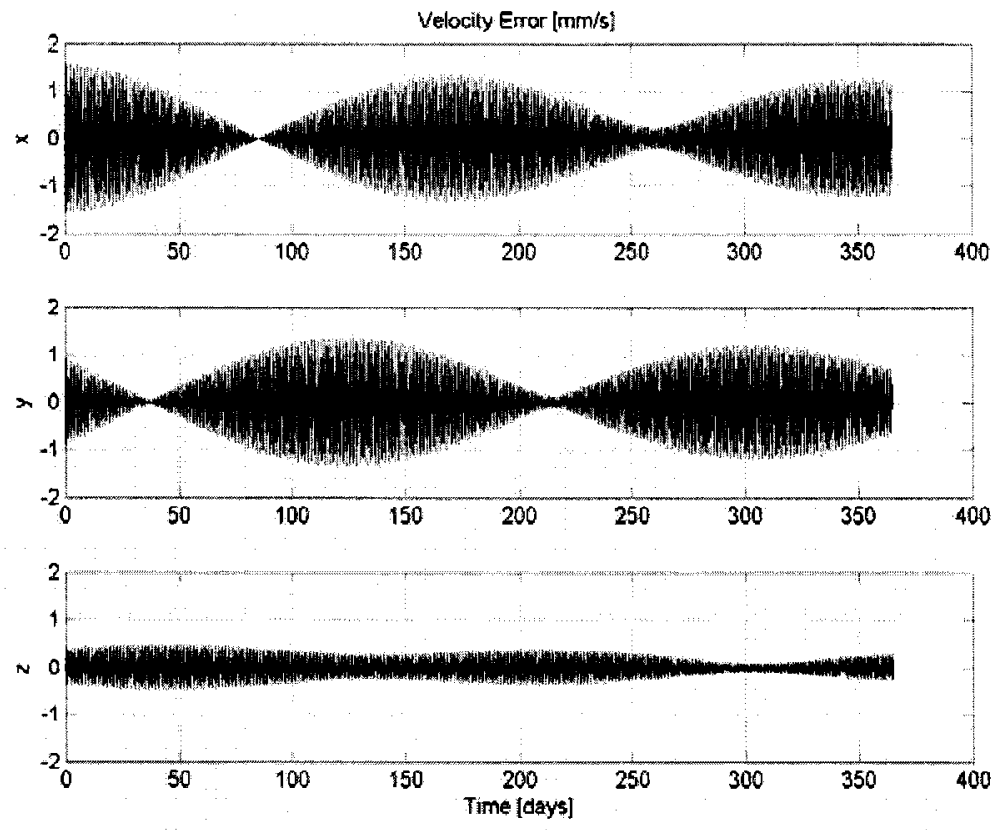

Figure $8.18-H_{\infty}$ velocity errors for 1 year (solar radiation pressure)

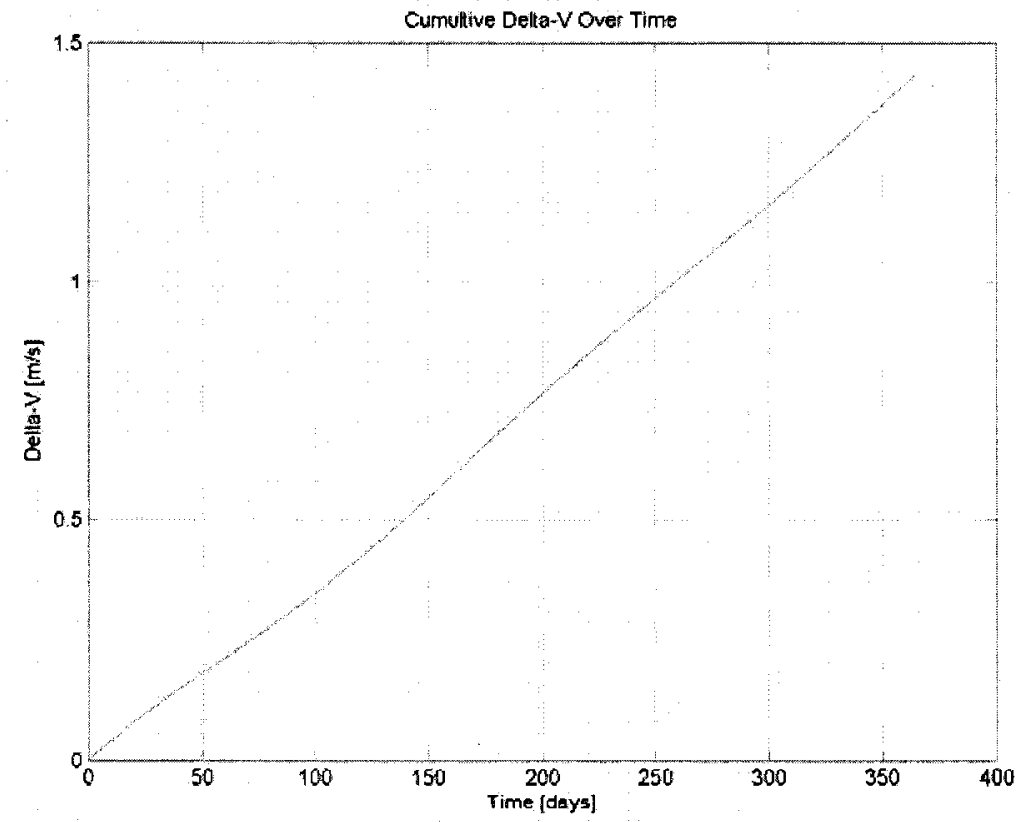

Figure 8.19 - $H_{\infty} \Delta v$ profile for 1 year (solar radiation pressure) 


\begin{tabular}{|c|c|c|c|c|}
\cline { 2 - 5 } \multicolumn{1}{c|}{} & \multicolumn{4}{c|}{ Maximum Errors } \\
\cline { 2 - 5 } \multicolumn{1}{c|}{} & $\mathbf{x}$ & $\mathbf{y}$ & $\mathbf{z}$ & \\
\hline position $[\mathrm{m}]$ & 10 & 8.7 & 3.2 & 1.43 \\
\hline velocity $[\mathrm{mm} / \mathrm{s}]$ & 1.5 & 1.4 & 0.49 & \\
\hline
\end{tabular}

Table 8.6 - $\mathbf{H}_{\infty}$ results for 1 year (solar radiation pressure)

Table 8.6 shows that the position errors are slightly larger than those of the unperturbed system, but they are still maintained within the allowance. However, the system still witnesses unacceptable oscillations.

\subsection{Measurement Noise Results}

Results pertaining to the $\mathrm{H}_{\infty}$ controller applied to the nonlinear system with the presence of measurement noise are shown in Figures 8.20 through 8.22. Numerical results are listed in Table 8.8 . 

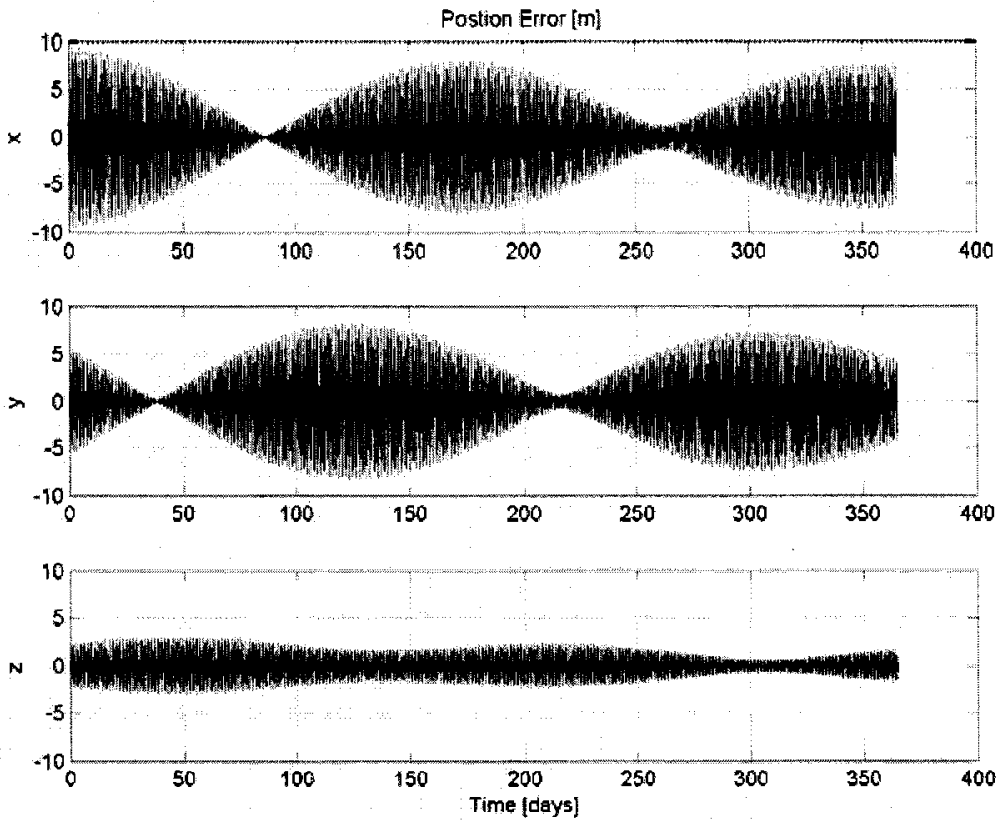

Figure 8.20 - $\mathbf{H}_{\infty}$ position errors for 1 year (measurement noise)
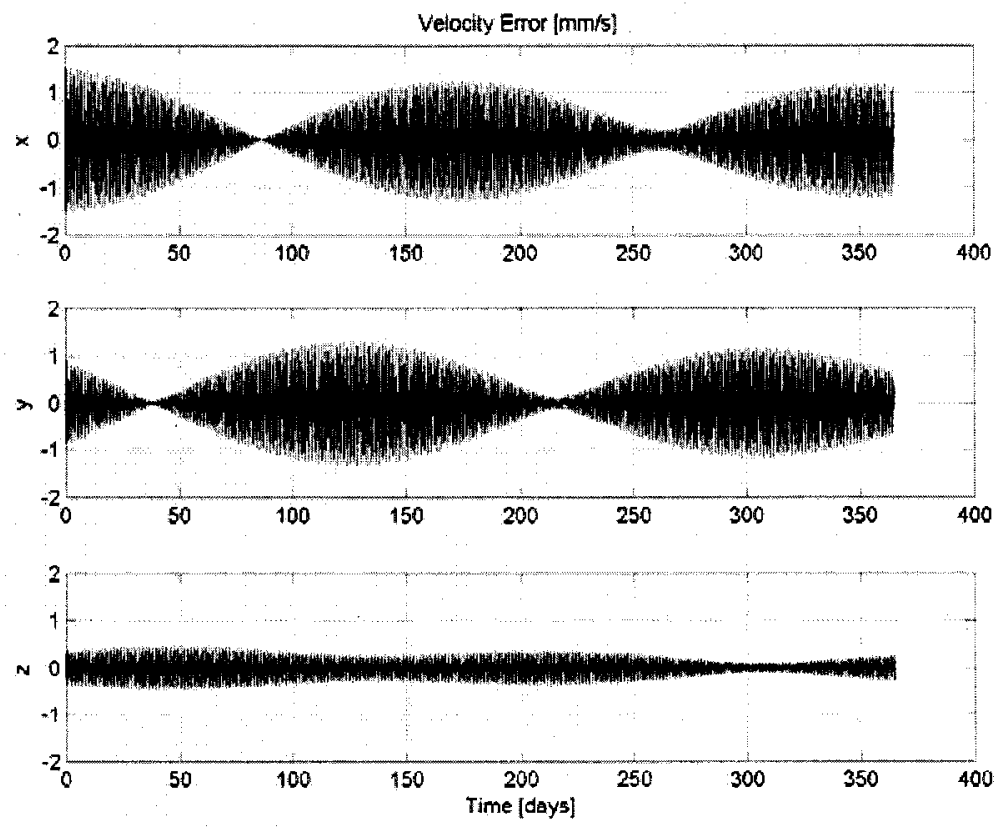

Figure 8.21 - $\mathbf{H}_{\infty}$ velocity errors for 1 year (measurement noise) 


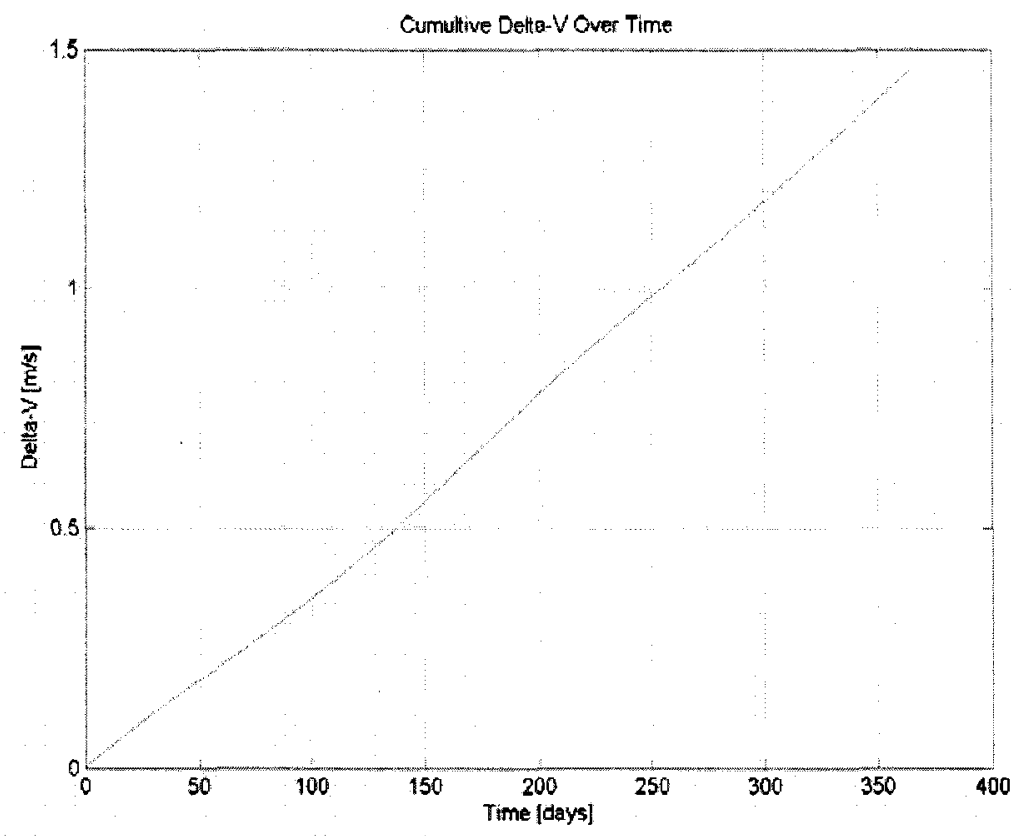

Figure 8.22 - $H_{\infty} \Delta v$ profile for 1 year (measurement noise)

\begin{tabular}{|c|c|c|c|c|}
\cline { 2 - 5 } \multicolumn{1}{c|}{} & \multicolumn{3}{c|}{ Maximum Errors } & \\
\cline { 2 - 5 } \multicolumn{1}{c|}{} & $\mathbf{x}$ & $\mathbf{y}$ & $\mathbf{2}$ & (m) \\
\hline position $[\mathbf{m}]$ & 9.3 & 8.2 & 2.9 & 1.46 \\
\hline velocity $[\mathbf{m m} / \mathbf{s}]$ & 1.5 & 1.4 & 0.47 & \\
\hline
\end{tabular}

Table 8.7 - $\mathbf{H}_{\infty}$ results for 1 year (measurement noise)

The resulting position errors and $\Delta v$ are equal to those of the unperturbed system, maintained within the allowed tolerances, although undesirable oscillations still exist. 


\subsection{Differing Initial Conditions Results}

Results pertaining to the $\mathrm{H}_{\infty}$ controller applied to the nonlinear system with incorrect initial conditions are shown in Figures 8.23 through 8.25. Numerical results are listed in Table 8.8.
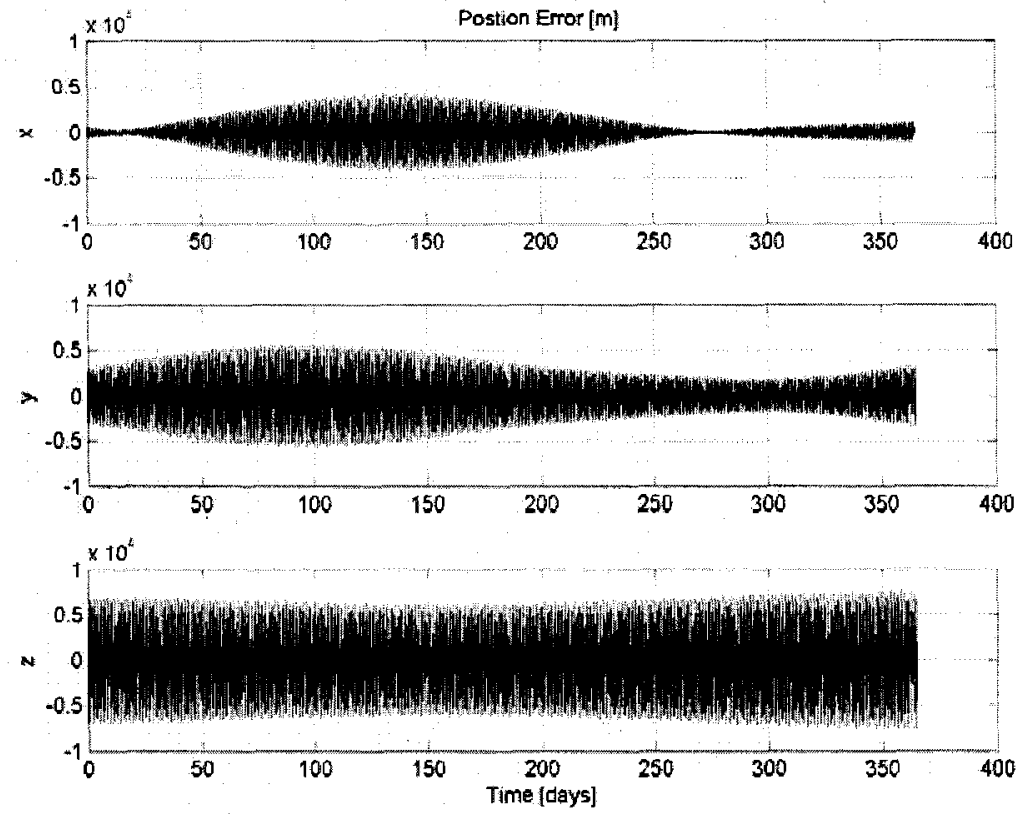

Figure 8.23 - $\mathbf{H}_{\infty}$ position errors for 1 year (differing initial conditions) 

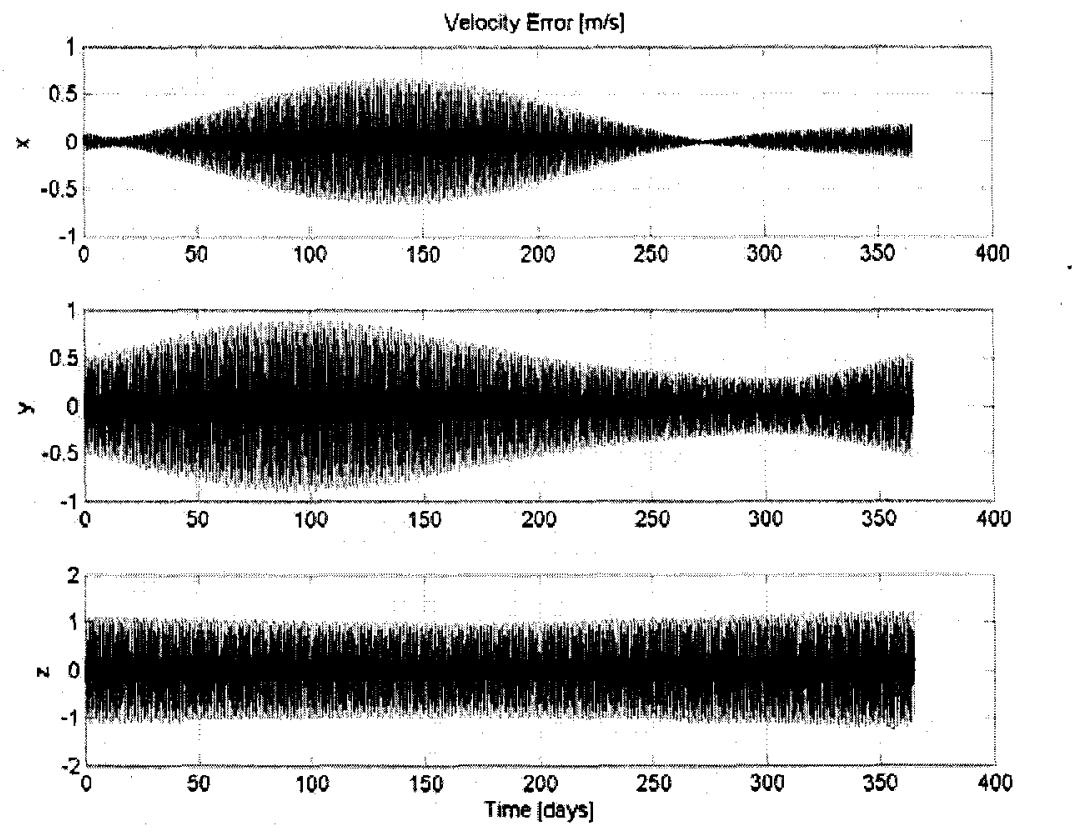

Figure 8.24 - $\mathbf{H}_{\infty}$ velocity errors for 1 year (differing initial conditions)

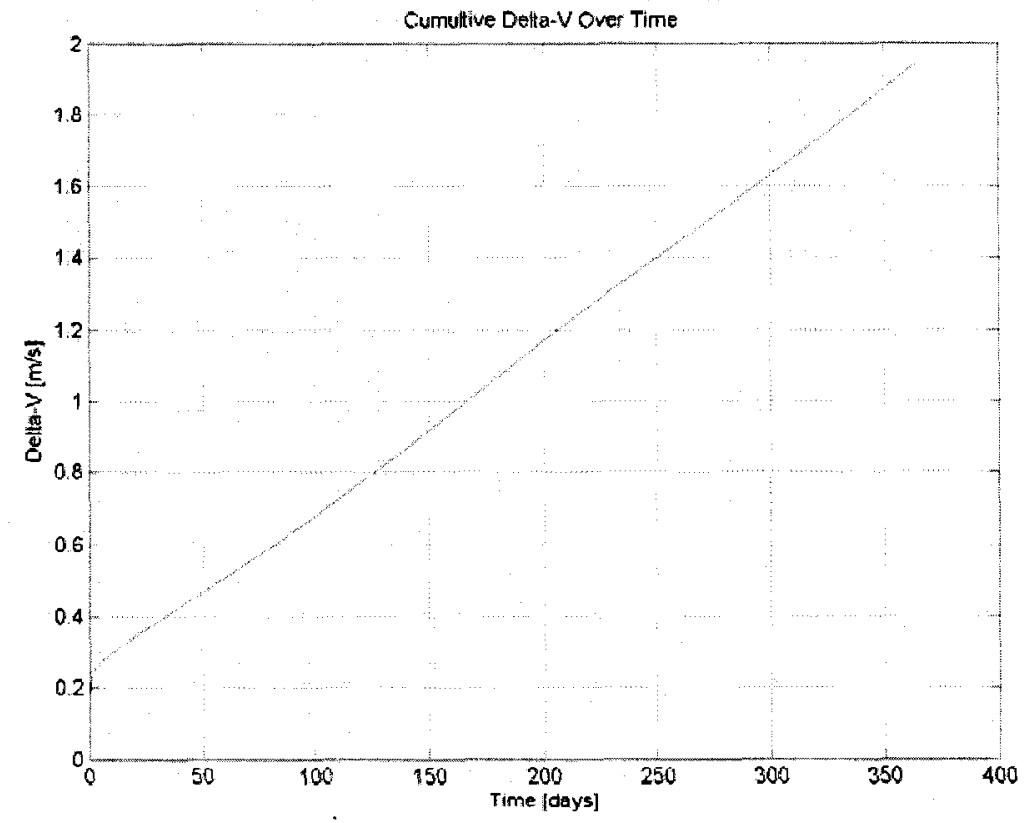

Figure 8.25 - $H_{\infty} \Delta v$ profile for 1 year (differing initial conditions) 


\begin{tabular}{|c|c|c|c|c|}
\cline { 2 - 5 } \multicolumn{1}{c|}{} & \multicolumn{3}{c|}{ Maximum Errors } & \\
\cline { 2 - 5 } \multicolumn{1}{c|}{} & $\mathbf{X}$ & $\mathbf{y}$ & $\mathbf{z}$ & \\
\hline posifion $[\mathrm{km}]$ & 4.2 & 5.6 & 7.6 & 1.95 \\
\hline velocity $[\mathrm{m} / \mathrm{s}]$ & 0.67 & 0.89 & 1.2 & 1.95 \\
\hline
\end{tabular}

Table 8.8 - $\mathrm{H}_{\infty}$ results for 1 year (differing initial conditions)

The $\mathrm{H}_{\infty}$ controller does not maintain stability with the presence of differing initial conditions. Both position and velocity errors increase by a factor of 1000 , greatly surpassing the mission tolerances (note Table 8.8 uses larger units). $\mathrm{H}_{\infty}$ is not designed to take differing initial conditions into account. Referring to the two-boundary problem that defines the controller, the maximization boundary adjusts for a maximum disturbance. A wrong initial condition can not be modeled as a disturbance, however, causing the controller to yield large errors. The control does, on the other hand, satisfy the minimization problem by keeping the $\Delta \mathrm{v}$ minimized. Nevertheless the errors are too large, establishing the controller to be susceptible to differing initial conditions.

\subsection{Worst Case Scenario Results}

Results pertaining to the $\mathrm{H}_{\infty}$ controller applied to the nonlinear system with the presence of all modeled disturbances and perturbations are shown in Figures 8.26 through 8.28. Numerical results are listed in Table 8.9. 

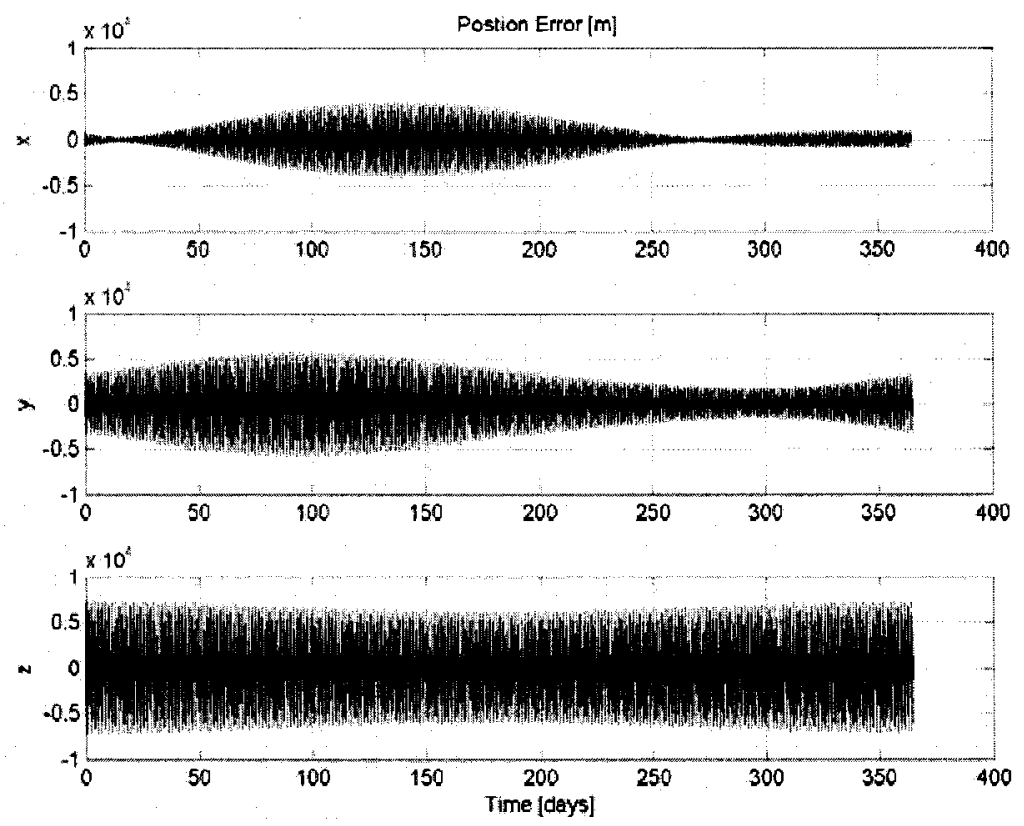

Figure 8.26 - $\mathbf{H}_{\infty}$ position errors for 1 year (worst case)
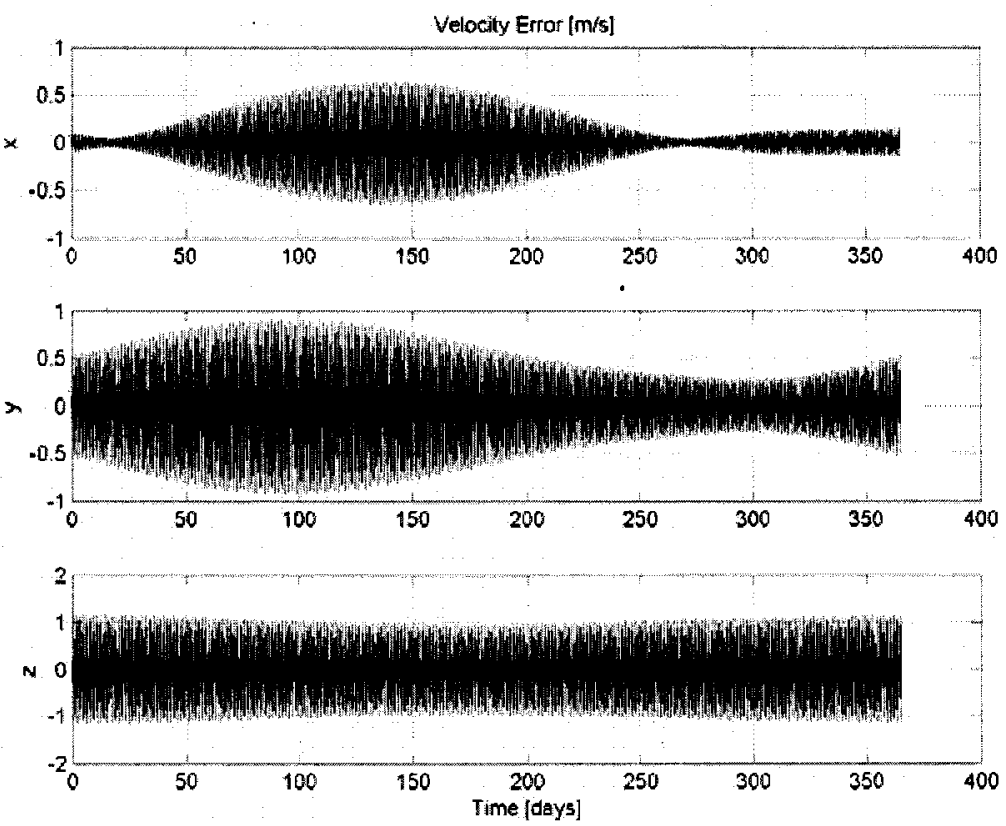

Figure 8.27 - $\mathbf{H}_{\infty}$ velocity errors for 1 year (worst case) 


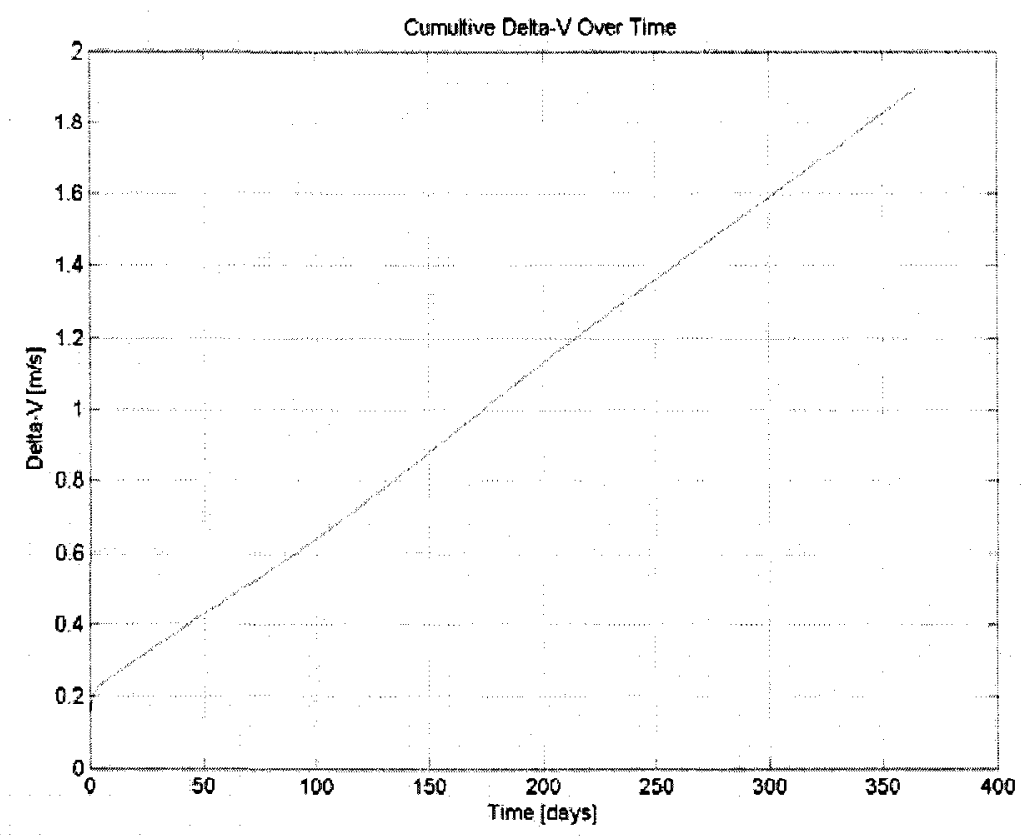

Figure $8.28-H_{\infty} \Delta v$ profile for 1 year (worst case)

\begin{tabular}{|c|c|c|c|c|}
\hline & \multicolumn{3}{|c|}{ Maximum Errors } & \multirow{2}{*}{ (mbl) } \\
\hline & $x^{x}$ & $y$ & 2 & \\
\hline position [km] & 4.1 & 5.8 & 7.3 & \multirow{2}{*}{1.9} \\
\hline velocity $[\mathrm{m} / \mathrm{s}]$ & 0.65 & 0.92 & 1.1 & \\
\hline
\end{tabular}

Table $8.9-\mathbf{H}_{\infty}$ results for 1 year (solar radiation pressure)

The results of the worst case scenario have large errors similar to those of the differing conditions (note Table 8.9 uses larger units). Since the system became unsatisfactory with differing initial conditions, it can only be expected that the worst case scenario would follow the same trend. 


\subsection{Summary}

\begin{tabular}{|c|c|c|c|c|}
\cline { 2 - 5 } \multicolumn{1}{c|}{} & \multicolumn{3}{c|}{ Max Position Errors $[\mathbf{m}]$} & \multicolumn{1}{c|}{} \\
\cline { 2 - 5 } \multicolumn{1}{c|}{} & $\mathbf{X}$ & $\mathbf{y}$ & $\mathbf{z}$ & $\mathbf{m}$ \\
\hline Unperturbed & 9.3 & 8.2 & 2.9 & 1.46 \\
\hline Thr. Misalign. & 9.4 & 8.4 & 2.9 & 1.46 \\
\hline Thr. Bias & 9.6 & 7.9 & 3.6 & 1.46 \\
\hline Self-Gravity & 9.3 & 8.2 & 2.9 & 1.46 \\
\hline Jupiter Gravity & 9.3 & 8.3 & 2.9 & 1.46 \\
\hline SRP & 10 & 8.7 & 3.2 & 1.43 \\
\hline Meas. Noise & 9.3 & 8.2 & 2.9 & 1.46 \\
\hline Difforing ICs & 4222 & 5653 & 7595 & 1.95 \\
\hline Worst Case & 4099 & 5831 & 7298 & 1.9 \\
\hline
\end{tabular}

Table 8.10 - $\mathbf{H}_{\infty}$ results (summary)

Reviewing Table 8.10, $\mathrm{H}_{\infty}$ provides acceptable errors for the majority of the cases. Aside from the unacceptable levels of oscillation, the controller performs well for each case investigated except for that of differing initial conditions, which produces errors much larger than those required by the mission. Differing initial conditions will definitely occur in an actual orbit. Therefore, between initial conditions and undesirable oscillations, $\mathrm{H}_{\infty}$ is assessed to not be robust enough to satisfy mission requirements. 


\section{CHAPTER 9}

\section{SLIDING MODE CONTROL}

\subsection{Fundamental Equations}

\subsubsection{Sliding Surface}

Sliding mode control (SMC) is a form of nonlinear control, which often utilizes two components to drive the tracking error to zero. One is an "equivalent" control, and is defined by the LQR controller in Chapter 6. The other control is what is known as the switching control term. The switching term is a function of the sliding surface, or hyperplane, defined as

$$
s_{i}\left(\tilde{x}_{i}, \dot{\tilde{x}}_{i}\right)=k \tilde{x}_{i}+\dot{\widetilde{x}}_{i}, \quad i=1,2,3
$$

where $\tilde{x}_{i}$ and $\dot{\widetilde{x}}_{i}$ are corresponding state errors and $k$ is a design parameter. Thus, when $s$ equals zero, the sliding surface passes through the origin seen in Figure 9.1. The sliding surface dynamics in Equation (9.1) are such that when $s\left(\widetilde{x}_{i}, \dot{\widetilde{x}}_{i}\right)=0$, the dynamics result in $k \widetilde{x}_{i}+\dot{\widetilde{x}}_{i}=0$. Solving this first order differential equation results in the solution $\tilde{x}_{i}(t)=\tilde{x}_{i}(0) e^{-k t}$. This converges to zero, so long as the error trajectory remains on the sliding surface. 


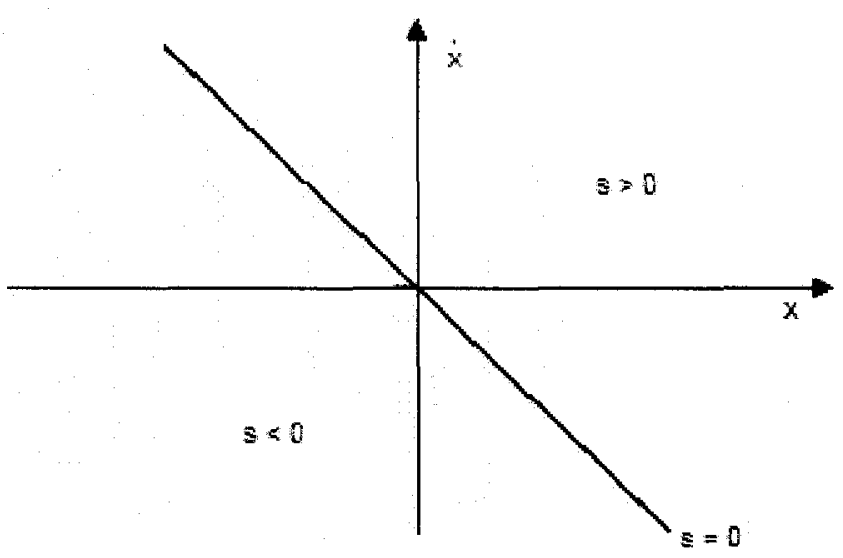

Figure 9.1 - Sliding surface hyperplane

To ensure that the system will reach this line, the following "reaching" condition is defined

$$
\dot{s}=-\delta \operatorname{sgn}(s)
$$

where sgn is the signum function

$$
\operatorname{sgn}(s)=\left\{\begin{array}{lll}
+1 & \text { if } & s>0 \\
-1 & \text { if } & s<0
\end{array}\right.
$$

and $\delta$ is positive design parameter. When the equivalent control term drives the error trajectory to the hyperplane, the reaching condition is satisfied, such that $s=0$, once on the sliding surface the switching control term ensures that the error trajectory remains on the sliding surface, despite the presence of any bounded perturbations or nonlinearities that may, otherwise, move the error trajectory off the sliding surface. The control is thus defined as

$$
u(t)=u_{L Q R}(t)-K \operatorname{sgn}(s(t))
$$




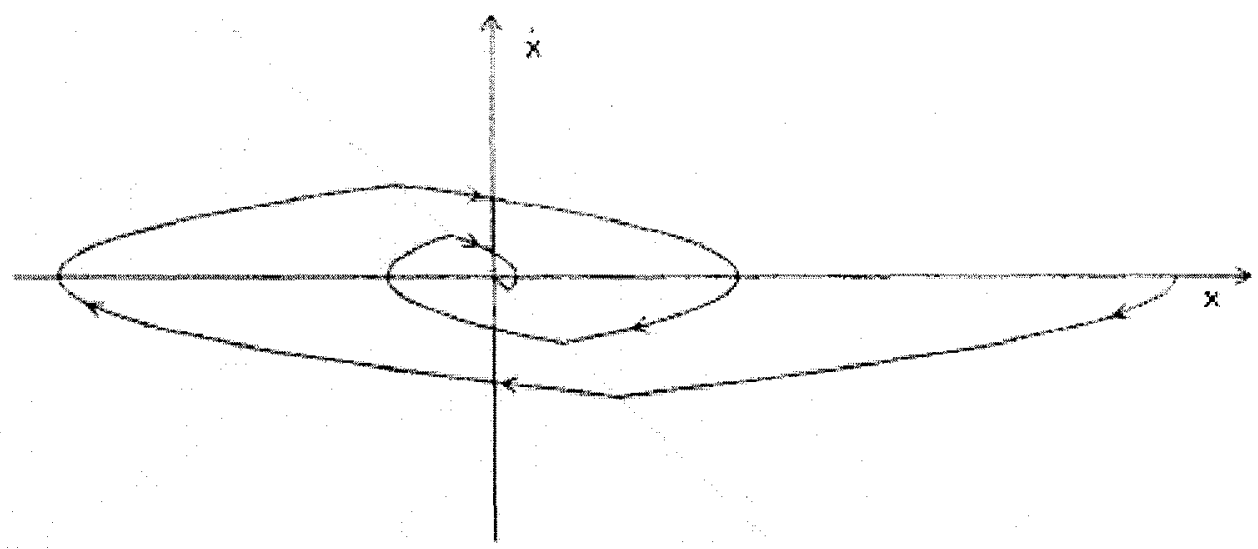

Figure 9.2 - SMC reaching condition [8]

\subsubsection{Sliding Surface Hyperplane Matrix}

Equation (9.1) can be written in matrix form as

$$
s_{i}(t)=\mathbf{g}_{i}^{T} \widetilde{\mathbf{x}}(t), i=1,2,3
$$

where $\mathbf{g}_{i}$ and $s_{i}$ are vectors of

$$
\begin{aligned}
\mathbf{G} & =\left[\mathbf{g}_{1}, \mathbf{g}_{2}, \mathbf{g}_{3}\right] \\
\mathbf{s} & =\left[s_{1}, s_{2}, s_{3}\right]
\end{aligned}
$$

$\mathrm{G} \in \mathbb{R}^{3 \times 6}$ is known as the hyperplane matrix. Equation (9.5) can be rewritten as

$$
\mathbf{s}(t)=\mathbf{G} \widetilde{\mathbf{x}}(t)
$$

which updates Equation (9.4) to

$$
\mathbf{u}(t)=\mathbf{u}_{e q}(t)-\boldsymbol{\eta} \operatorname{sgn}(\mathbf{G} \widetilde{\mathbf{x}}(t))
$$

where $\delta$ is replaced by $\boldsymbol{\eta} \in \mathbb{R}^{3 \times 3}$, a diagonal matrix with positive elements. 


\subsubsection{Optimal Sliding Mode Control}

The SMC has a form that takes advantage of the Riccati equation, known as Optimal Sliding Mode Control (OSMC). OSMC can be used to obtain an optimal hyperplane matrix ${ }^{2}$. The cost function to be minimized may be defined as

$$
J=\int_{t_{0}}^{t} \widetilde{\mathbf{x}}(\tau)^{T} \mathbf{Q} \widetilde{\mathbf{x}}(\tau) d \tau
$$

where the weighting matrix, $\mathbf{Q} \in \mathbb{R}^{6 \times 6}$, such that

$$
\mathbf{Q}=\left[\begin{array}{ll}
\mathbf{Q}_{r} & \mathbf{N} \\
\mathbf{N} & \mathbf{R}
\end{array}\right], \quad \mathbf{Q}=\mathbf{Q}^{T}
$$

and $\mathbf{N} \in \mathbb{R}^{3 \times 3}$ is the null space matrix of the control state-space matrix, $\mathbf{B}$. The null space of $\mathbf{B}$ for the system, however, is equal to zero. Therefore, it will be omitted from the remaining equations. The input term, $\mathbf{u}$, is not present in the cost function since the system is subjected to the constraint

$$
\mathbf{s}(t)=\mathbf{G} \widetilde{\mathbf{x}}(t)=0
$$

Updating Equation (9.10)

$$
J=\int_{t_{0}}^{t} \widetilde{\mathbf{x}}_{1}(\tau)^{T} \mathbf{Q}_{r} \widetilde{\mathbf{x}}_{1}(\tau)+\widetilde{\mathbf{x}}_{2}(\tau)^{T} \mathbf{R} \widetilde{\mathbf{x}}_{2}(\tau) d \tau
$$

where

$$
\dot{\mathbf{x}}=\mathbf{A x}+\mathbf{B u}=\left[\begin{array}{ll}
\mathbf{A}_{11} & \mathbf{A}_{12} \\
\mathbf{A}_{21} & \mathbf{A}_{22}
\end{array}\right]\left[\begin{array}{l}
\mathbf{x}_{1} \\
\mathbf{x}_{2}
\end{array}\right]+\left[\begin{array}{c}
0 \\
\mathbf{B}_{2}
\end{array}\right] \mathbf{u}
$$

\footnotetext{
${ }^{2}$ Note: [3] uses a state-transformation in its analysis of SMC and OSMC. A transformation is not needed for the system in study, since it is already in the ideal form.
} 
The gain matrix, $\mathbf{K} \in \mathbb{R}^{3 \times 3}$, which will minimize $J$ is

$$
\mathbf{K}=\mathbf{R}^{-1} \mathbf{A}_{12}^{T} \mathbf{P}
$$

where $\mathbf{P} \in \mathbb{R}^{3 \times 3}$ is the solution to the steady-state Riccati equation

$$
\mathbf{P} \mathbf{A}_{11}+\mathbf{A}_{11}^{T} \mathbf{P}-\mathbf{P} \mathbf{A}_{12} \mathbf{R}^{-1} \mathbf{A}_{12}^{T} \mathbf{P}+\mathbf{Q}_{r}=0
$$

The hyperplane matrix can then be formed by

$$
\mathbf{G}=\left[\begin{array}{ll}
\mathbf{K} & \mathbf{I}_{3}
\end{array}\right]
$$

\subsubsection{Chattering Effect}

While the tracking error is being driven to zero, the system may oscillate about the sliding surface due to the hard nonlinearity of the switching control term, as seen in Figure 9.3. This is known as the chattering effect. To decrease chattering the signum function can be replaced by a saturation function, resulting in a boundary layer of thickness, $\varphi$, as seen in Figure 9.4.

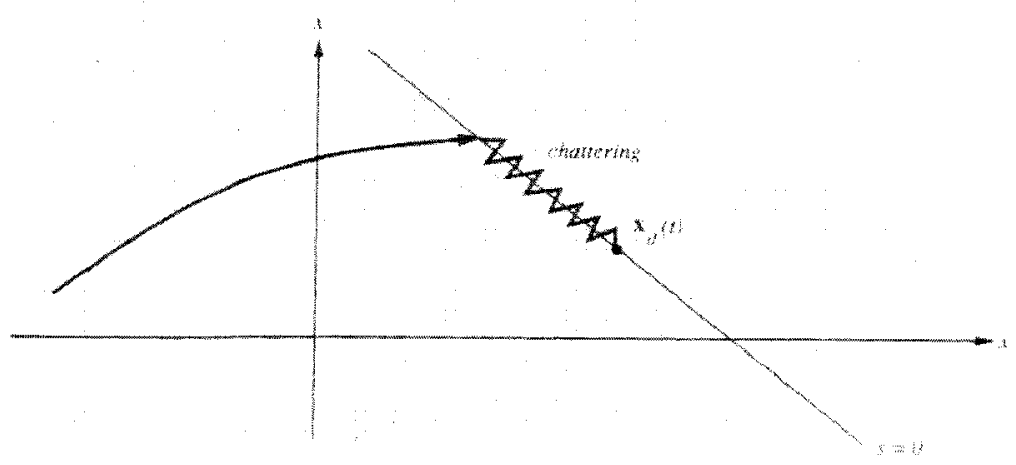

Figure 9.3 - Chattering effect [6] 


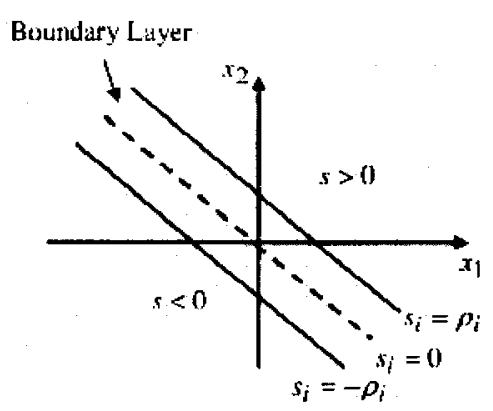

Figure 9.4 - Boundary layer [3]

The new control becomes

$$
\mathbf{u}=\mathbf{K}_{L Q R} \widetilde{\mathbf{x}}+\operatorname{diag}(\boldsymbol{\eta}) \operatorname{sat}(\mathbf{G} \widetilde{\mathbf{x}})
$$

where

$$
\operatorname{sat}(s)=\left\{\begin{array}{ccc}
\operatorname{sgn}(s) & \text { if } & |s|>1 \\
\frac{s}{\varphi} & \text { otherwise }
\end{array}\right.
$$

The simplified block diagram can be seen in Figure 9.5 .

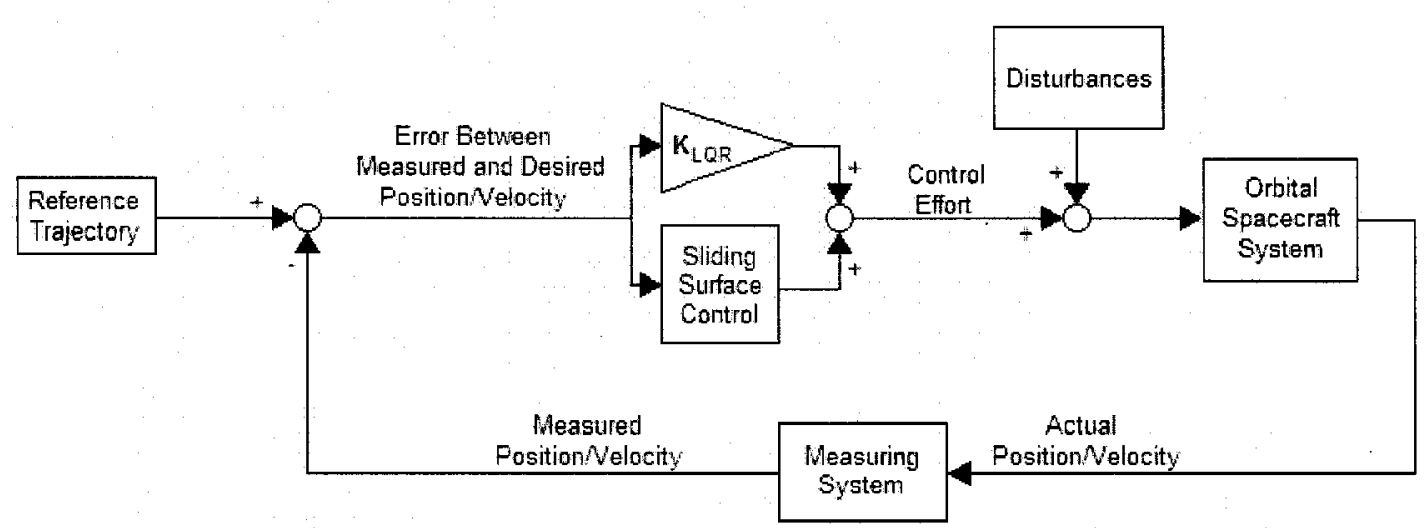

Figure 9.5 - Simplified SMC block diagram 


\subsection{Gain Selection}

Like the previous controllers, gain selection begins with the linearized CR3BP equations. Intuitively trial and error is used to choose the gains of the $\mathbf{Q}, \boldsymbol{\eta}$, and $\boldsymbol{\varphi}$ until an acceptable transient response is obtained. The chosen gains are applied to the nonlinear system and tuned through intuitive deduction until the following gains are chosen:

The optimal weighting matrix is chosen to be

$$
\mathbf{Q}=\left[\begin{array}{cccccc}
9 \cdot 10^{-5} & 0 & 0 & 0 & 0 & 0 \\
0 & 10^{-4} & 0 & 0 & 0 & 0 \\
0 & 0 & 7 \cdot 10^{-6} & 0 & 0 & 0 \\
0 & 0 & 0 & 9 \cdot 10^{-5} & 0 & 0 \\
0 & 0 & 0 & 0 & 4 \cdot 10^{-5} & 0 \\
0 & 0 & 0 & 0 & 0 & 10^{-4}
\end{array}\right]
$$

which yields a steady-state Riccati equation solution of

$$
\mathbf{P}=\left[\begin{array}{ccc}
9 \cdot 10^{-5} & 0 & 0 \\
0 & 6.3 \cdot 10^{-5} & 0 \\
0 & 0 & 2.6 \cdot 10^{-5}
\end{array}\right]
$$

and the gain matrix

$$
\mathbf{K}=\left[\begin{array}{ccc}
1 & 0 & 0 \\
0 & 1.6 & 0 \\
0 & 0 & 0.26
\end{array}\right]
$$

Thus the hyperplane becomes

$$
\mathbf{G}=\left[\begin{array}{cccccc}
1 & 0 & 0 & 1 & 0 & 0 \\
0 & 1.6 & 0 & 0 & 1 & 0 \\
0 & 0 & 0.26 & 0 & 0 & 1
\end{array}\right]
$$


The design parameter, $\boldsymbol{\eta}$, is selected to be

$$
\boldsymbol{\eta}=\left[\begin{array}{ccc}
5 \cdot 10^{-5} & 0 & 0 \\
0 & 4 \cdot 10^{-5} & 0 \\
0 & 0 & 2 \cdot 10^{-4}
\end{array}\right]
$$

and the boundary layer thickness is set to $\varphi=2$.

\subsection{Unperturbed System Results}

Results pertaining to the SMC controller applied to the nonlinear system without disturbances or perturbations are shown in Figures 9.6 through 9.8.
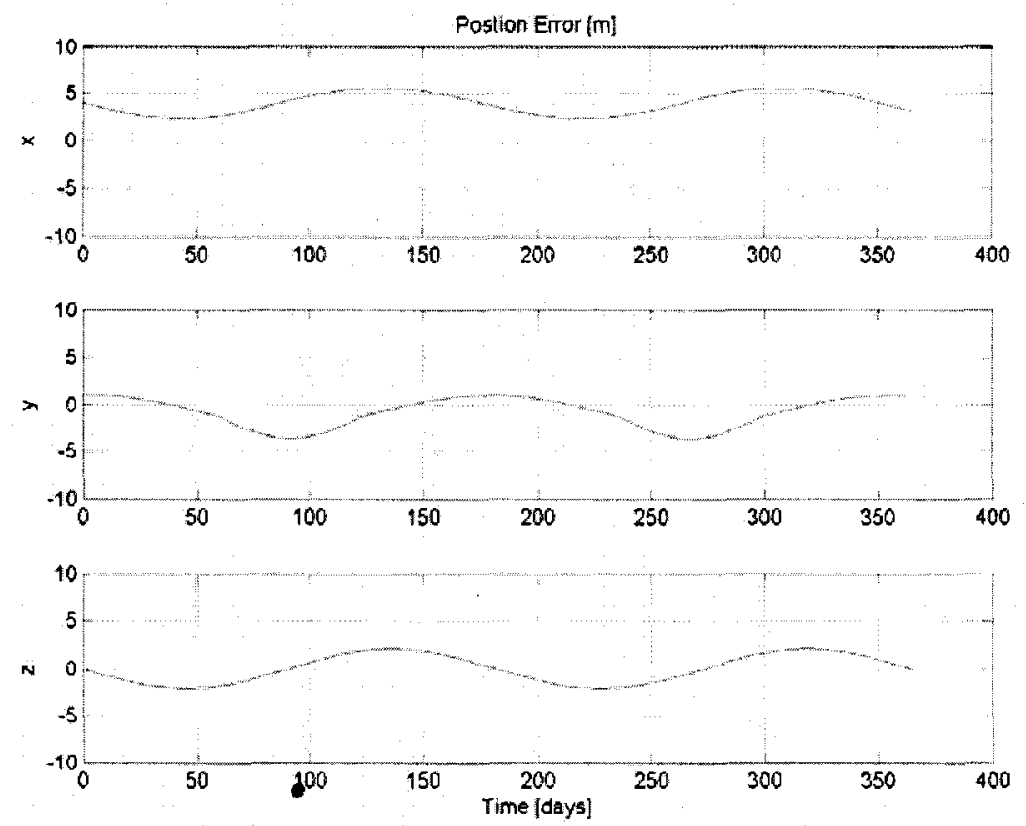

Figure 9.6 - SMC position errors for 1 year (unperturbed) 

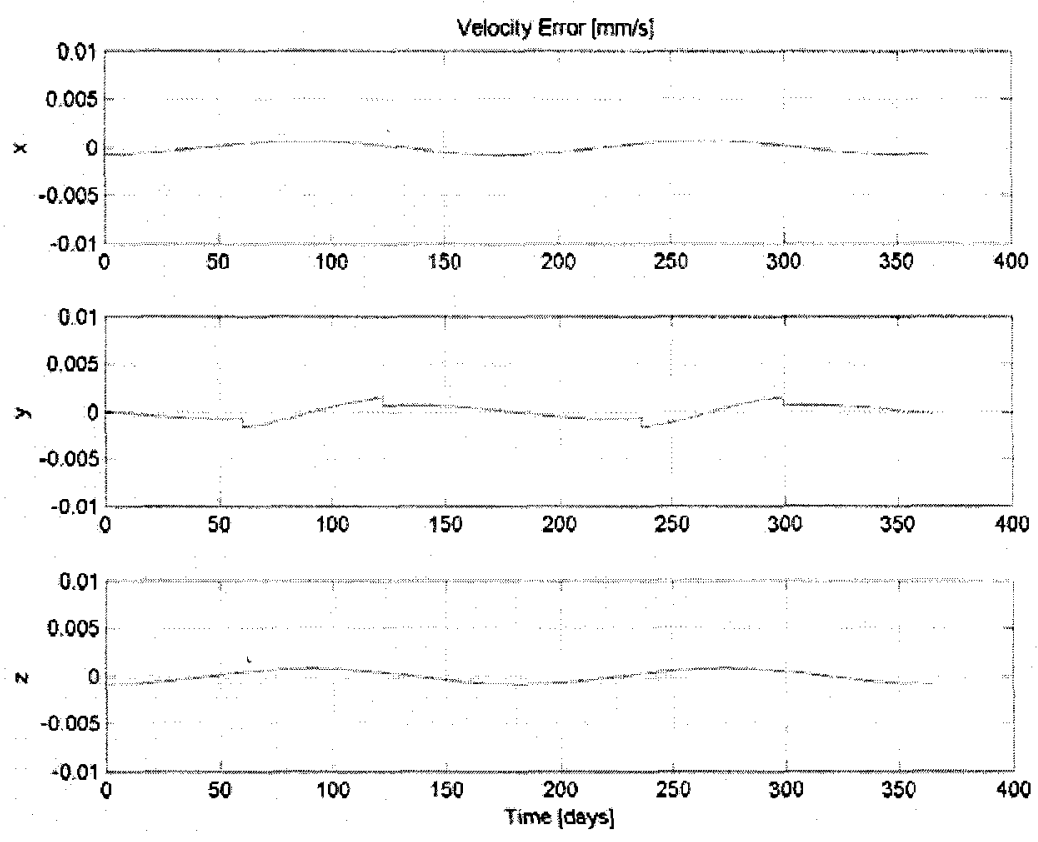

Figure 9.7 - SMC velocity errors for 1 year (unperturbed)

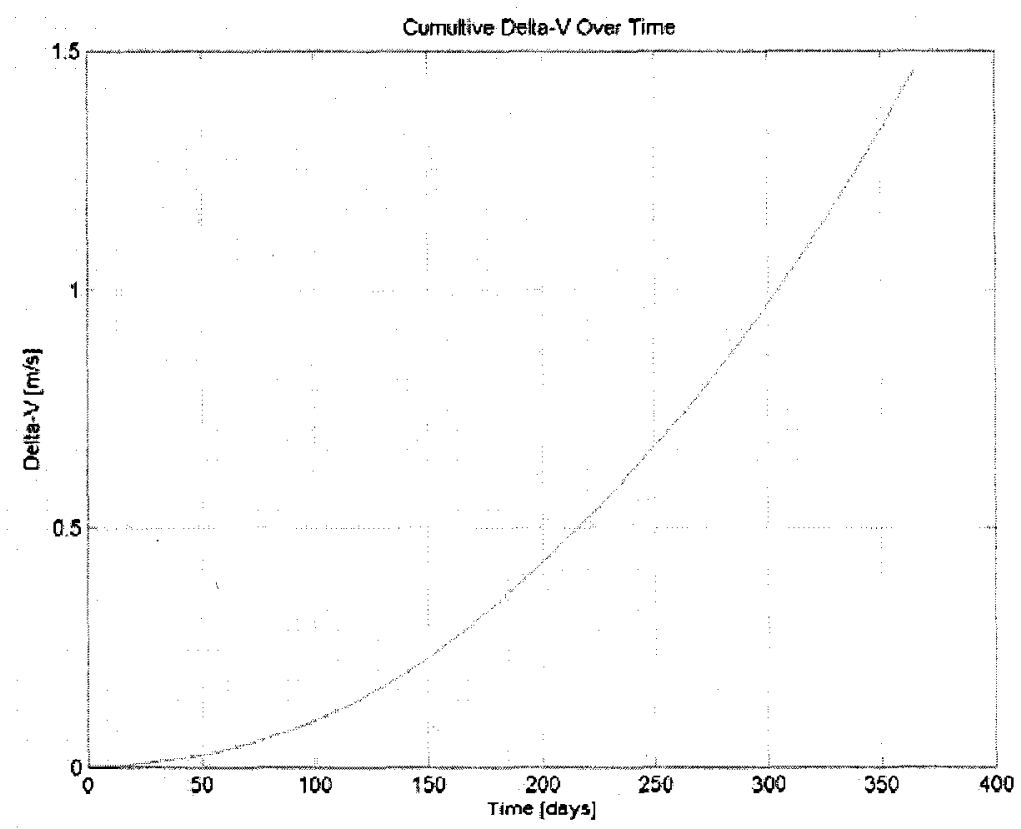

Figure 9.8 - SMC $\Delta v$ profile for 1 year (unperturbed) 


\begin{tabular}{|c|c|c|c|c|}
\cline { 2 - 5 } \multicolumn{1}{c|}{} & \multicolumn{4}{c|}{ Maximum Erors } \\
\hline position $[\mathbf{m}]$ & $\mathbf{X}$ & $\mathbf{y}$ & $\mathbf{z}$ & \\
\hline velocity $[\mathrm{mm} / \mathrm{s}]$ & 0.69 & 3.6 & 2 & 1.46 \\
\hline
\end{tabular}

Table 9.1 - SMC results for 1 year (unperturbed)

Table 9.1 lists the $\Delta v$ and maximum errors for position and velocity once the orbit is stabilized. The controller performs very well, maintaining errors well within the allowable tolerances. The position error in the $\mathrm{x}$-direction is maintained but produces a bias of five meters, which is due to the LQR controller. As in the discussed in the Chapter 7, decreasing the bias would require a larger control effort, although the bias is allowable since the error maintains with in the tolerance. The effect of the SMC can be seen well in Figure 9.7, where the error sharply jumps, forming a tooth-like spike. This spike is when the sliding term, $s$, moves from one side of the hyperplane to the other, causing the saturation function to switch signs. The affect of this on the position error can be seen when the curvature of the error oscillation slightly changes. Most importantly, though, $\Delta \mathrm{v}$ is kept to a minimal value while maintaining the trajectory. The $\Delta \mathrm{v}$ profile in Figure 9.4 is slightly different than those of the previous controllers. The control effort exponentially increases until it reaches a linear state. This is the controller slowly using more control effort to reach the hyperplane, but once the reaching function is satisfied, the $\Delta v$ remains at a near constant value equal to that of past controllers. 


\subsection{Thruster Misalignment Results}

Results pertaining to the SMC controller applied to the nonlinear system with thruster misalignments of a tenth of a degree are shown in Figures 9.9 through 9.11. Numerical results are listed in Table 9.2.
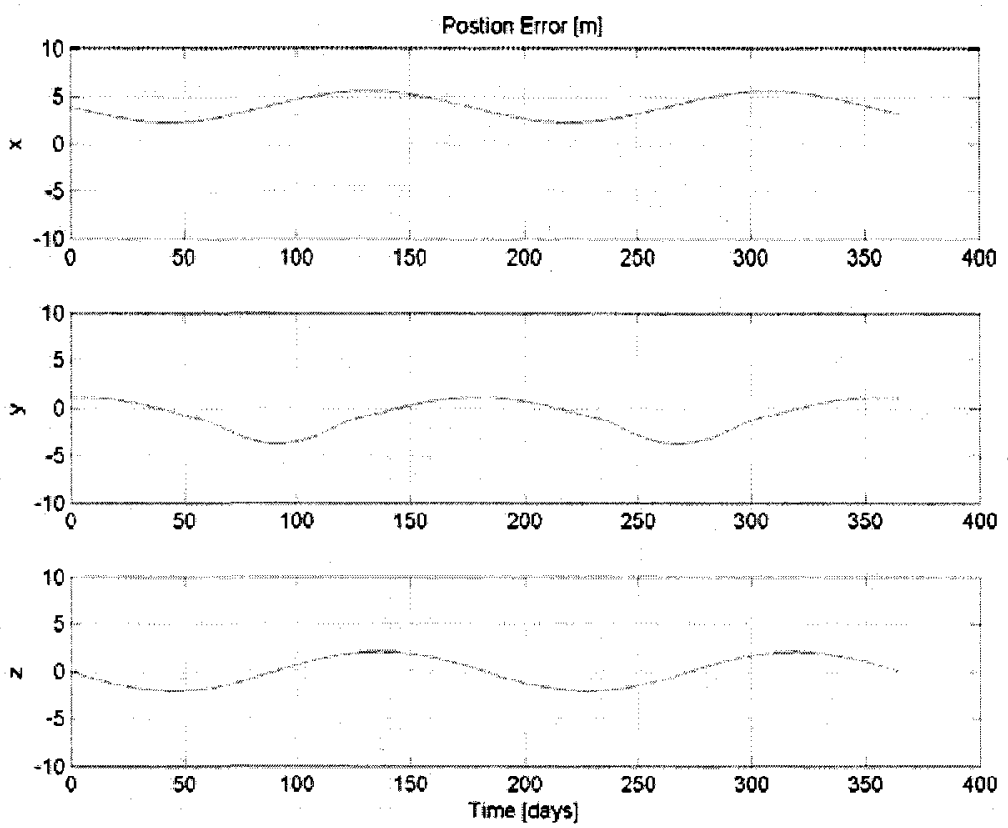

Figure 9.9 - SMC position errors for 1 year (thruster misalignments) 

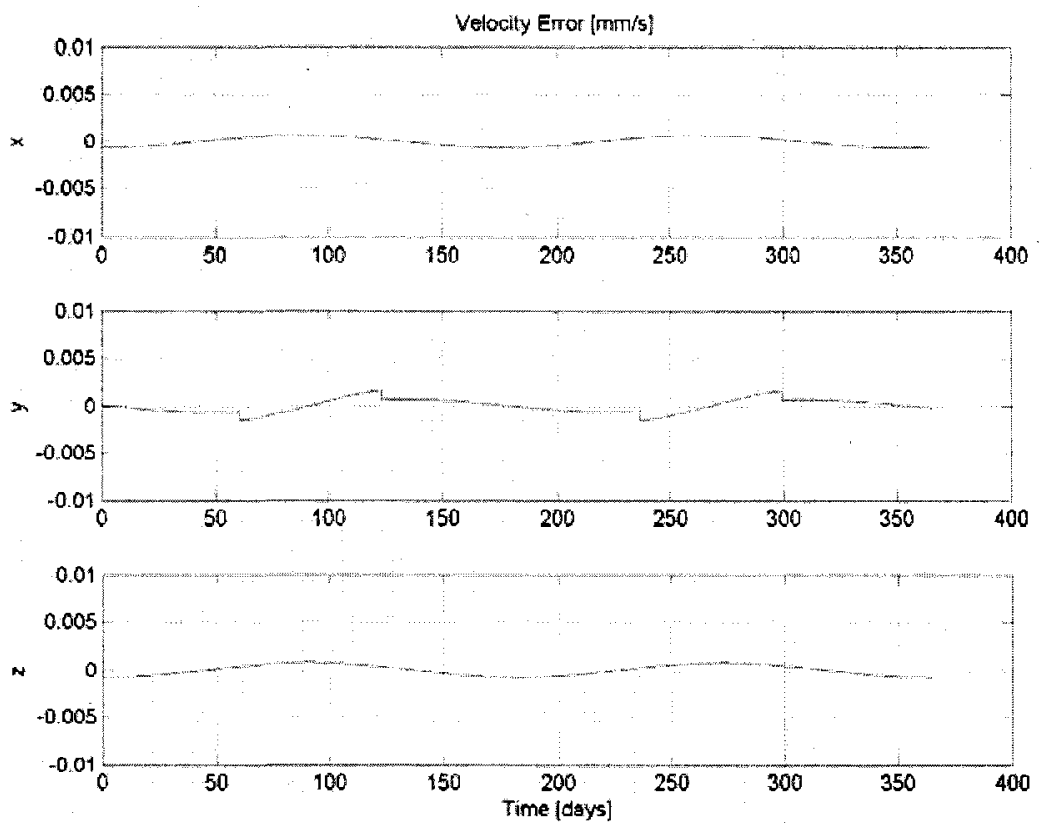

Figure 9.10 - SMC velocity errors for 1 year (thruster misalignments)

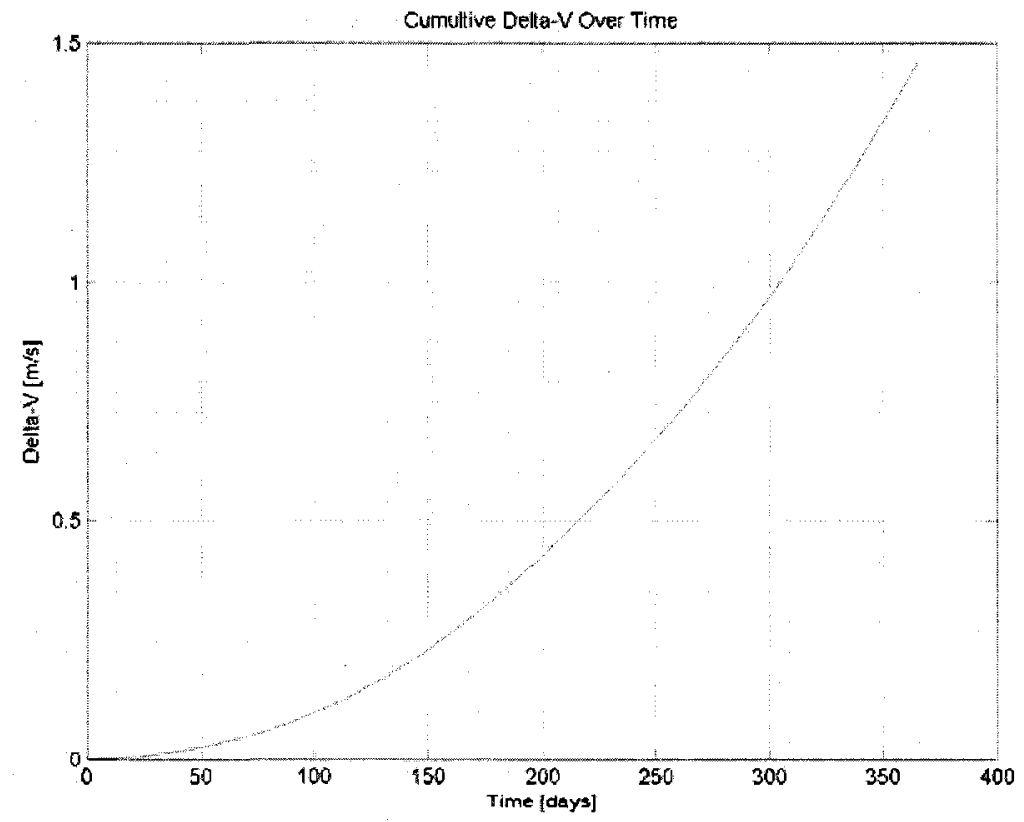

Figure 9.11 - SMC $\Delta \mathrm{v}$ profile for 1 year (thruster misalignments) 


\begin{tabular}{|c|c|c|c|c|}
\hline & \multicolumn{3}{|c|}{ Maximum Errors } & \multirow{2}{*}{ 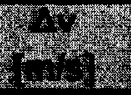 } \\
\hline & $x$ & $y$ & 2 & \\
\hline position $\mathrm{m}$ & 5.6 & 3.6 & 2 & \multirow{2}{*}{1.46} \\
\hline velocity $[\mathrm{pm} / \mathrm{s}]$ & 0.69 & 1.6 & 0.83 & \\
\hline
\end{tabular}

Table 9.2 - SMC results for 1 year (thruster misalignments)

The controller performs well, maintaining the same errors as the unperturbed system and maintaining the minimal $\Delta \mathrm{v}$. The error levels used are shown to be insignificant against SMC.

\subsection{Thruster Bias Results}

Results pertaining to the SMC controller applied to the nonlinear system with thruster biases of $-5 \%$ are shown in Figures 9.12 through 9.14 . Numerical results are listed in Table 9.3. 

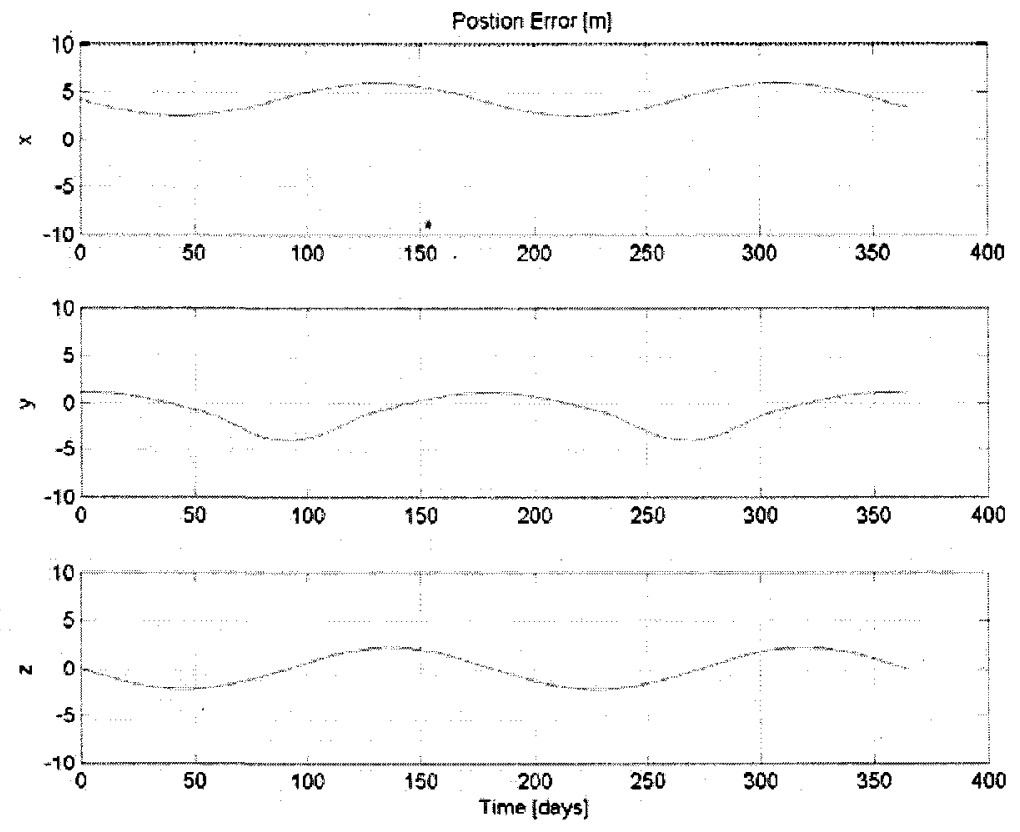

Figure 9.12 - SMC position errors for 1 year (thruster bias)
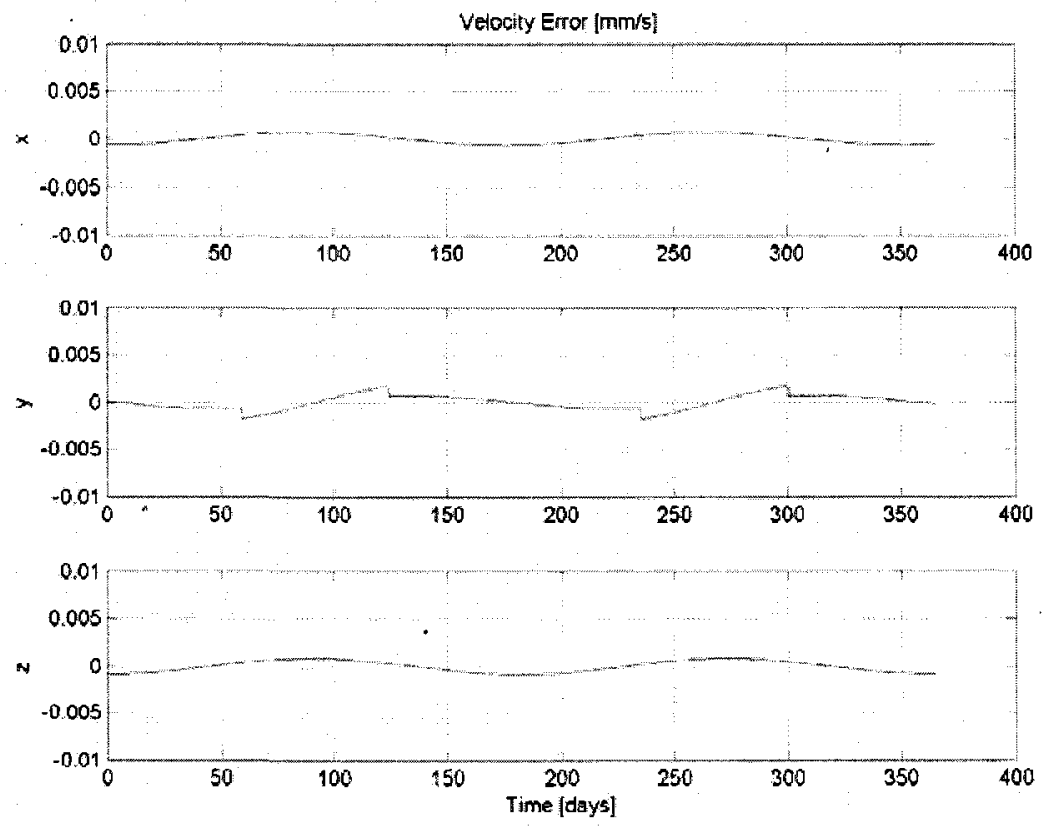

Figure 9.13 - SMC velocity errors for 1 year (thruster bias) 


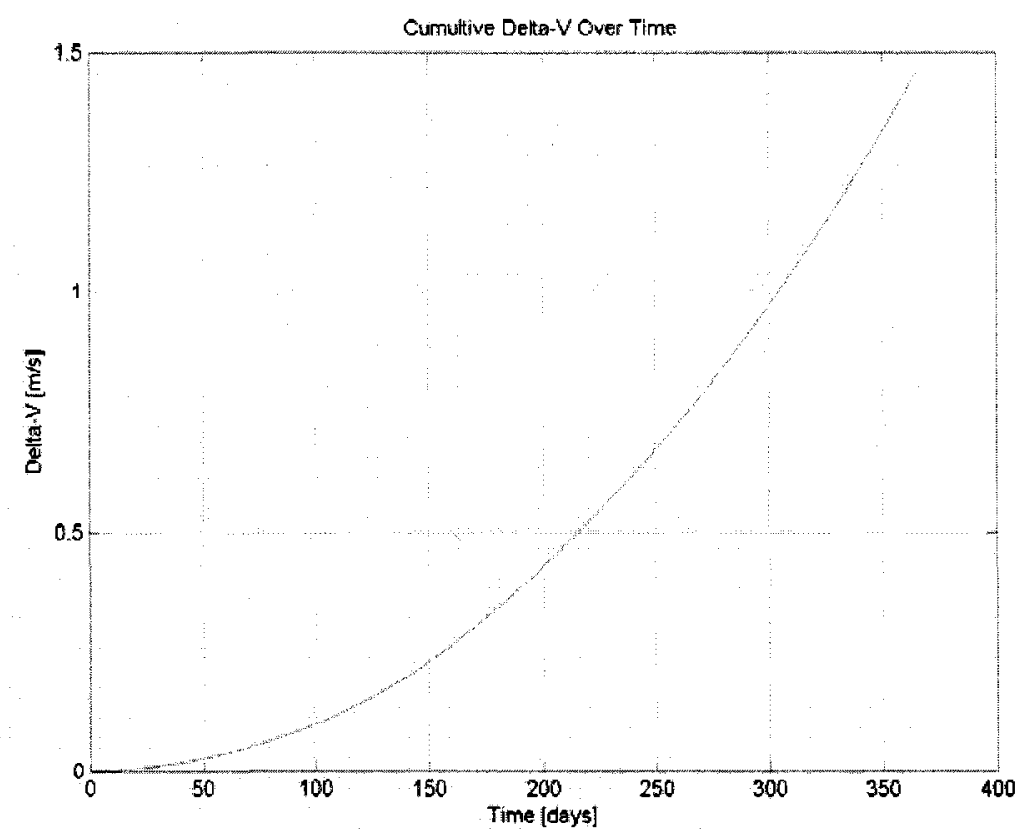

Figure 9.14 - SMC $\Delta \mathrm{v}$ profile for 1 year (thruster bias)

\begin{tabular}{|c|c|c|c|c|}
\hline & \multicolumn{3}{|c|}{ Maximum Errors } & \multirow{2}{*}{$\begin{array}{l}\text { Ay } \\
\text { rmsi }\end{array}$} \\
\hline & $x$ & $y$ & 2 & \\
\hline position $[\mathrm{m}]$ & 6 & 3.9 & 2.1 & \multirow{2}{*}{1.46} \\
\hline velocity $[\mathrm{pm} / \mathrm{s}]$ & 0.72 & $\overline{1.7}$ & 0.87 & \\
\hline
\end{tabular}

Table 9.3 - SMC results for 1 year (thruster bias)

The controller is effective, yielding errors just slightly larger, but still acceptable, than the unperturbed system and maintaining the minimal $\Delta \mathrm{v}$. The SMC controller is shown to provide acceptable results against the thruster bias levels defined. 


\subsection{Self-Gravity Results}

Results pertaining to the SMC controller applied to the nonlinear system with self-gravity from the leader satellite are shown in Figures 9.15 through 9.17. Numerical results are listed in Table 9.4.
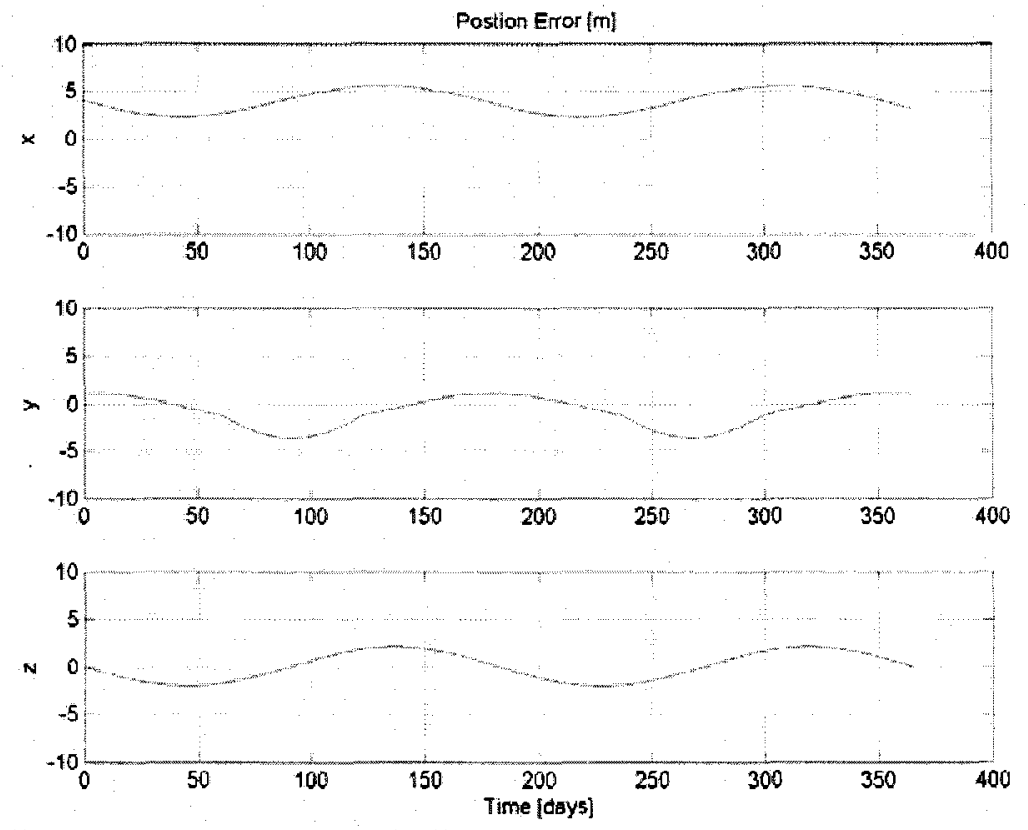

Figure 9.15 - SMC position errors for 1 year (self-gravity) 

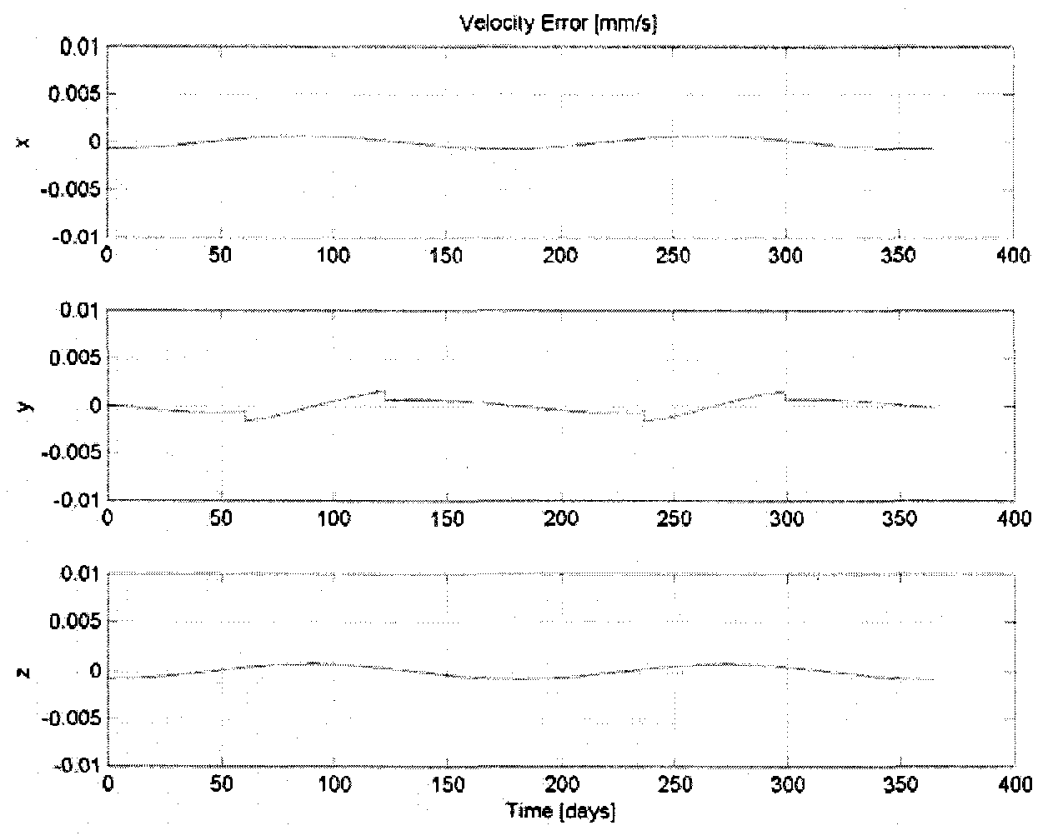

Figure 9.16 - SMC velocity errors for 1 year (self-gravity)

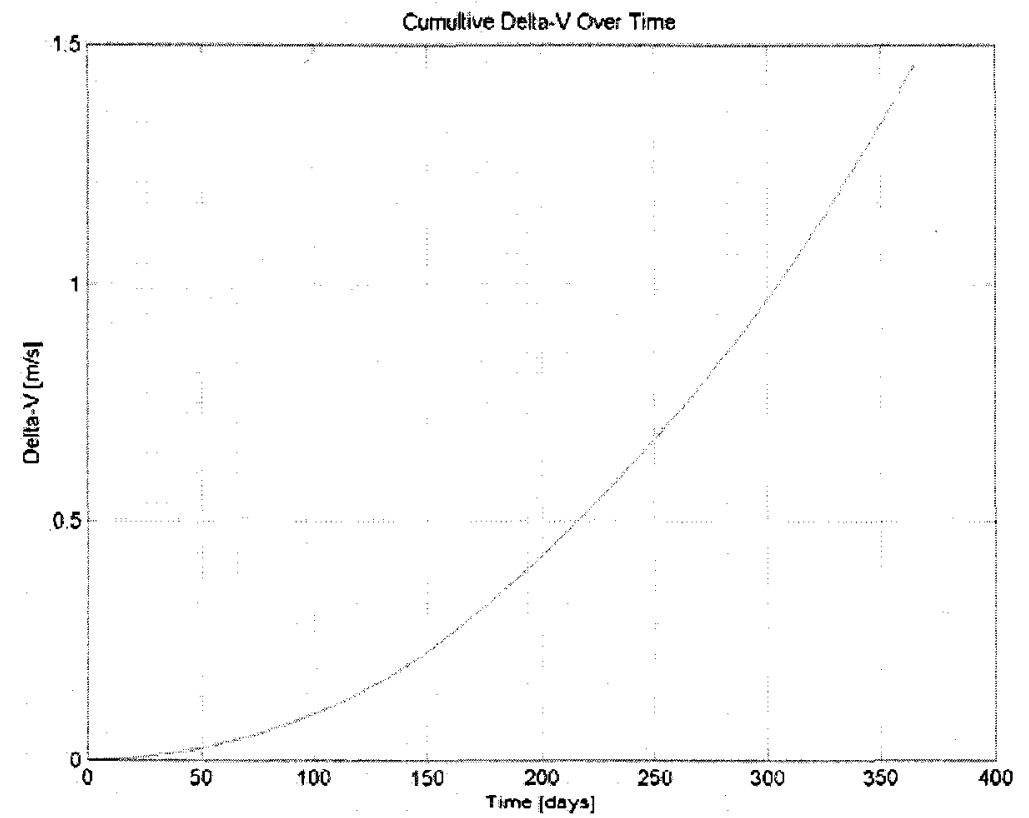

Figure 9.17 - SMC $\Delta v$ profile for 1 year (self-gravity) 


\begin{tabular}{|c|c|c|c|c|}
\cline { 2 - 5 } \multicolumn{1}{c|}{} & \multicolumn{3}{c|}{ Maximum Emors } & Wy \\
\cline { 2 - 5 } \multicolumn{1}{c|}{} & $\mathbf{x}$ & $\mathbf{y}$ & $\mathbf{z}$ & $\mathbf{2}$ \\
\hline position $[\mathrm{m}]$ & 5.6 & 3.6 & 1.46 \\
\hline velocity [um]s] & 0.69 & 1.6 & 0.83 & 1.46 \\
\hline
\end{tabular}

Table 9.4 - SMC results for 1 year (self-gravity)

Table 9.4 shows the controller produces the same low errors seen in the unperturbed system, while maintaining the minimal $\Delta v$. It is shown that self-gravity from the leader has negligible effects on the SMC.

\subsection{Jupiter Gravity Results}

Results pertaining to the SMC controller applied to the nonlinear system while experiencing gravitational forces due to Jupiter are shown in Figures 9.18 through 9.20. Numerical results are listed in Table 9.5. 

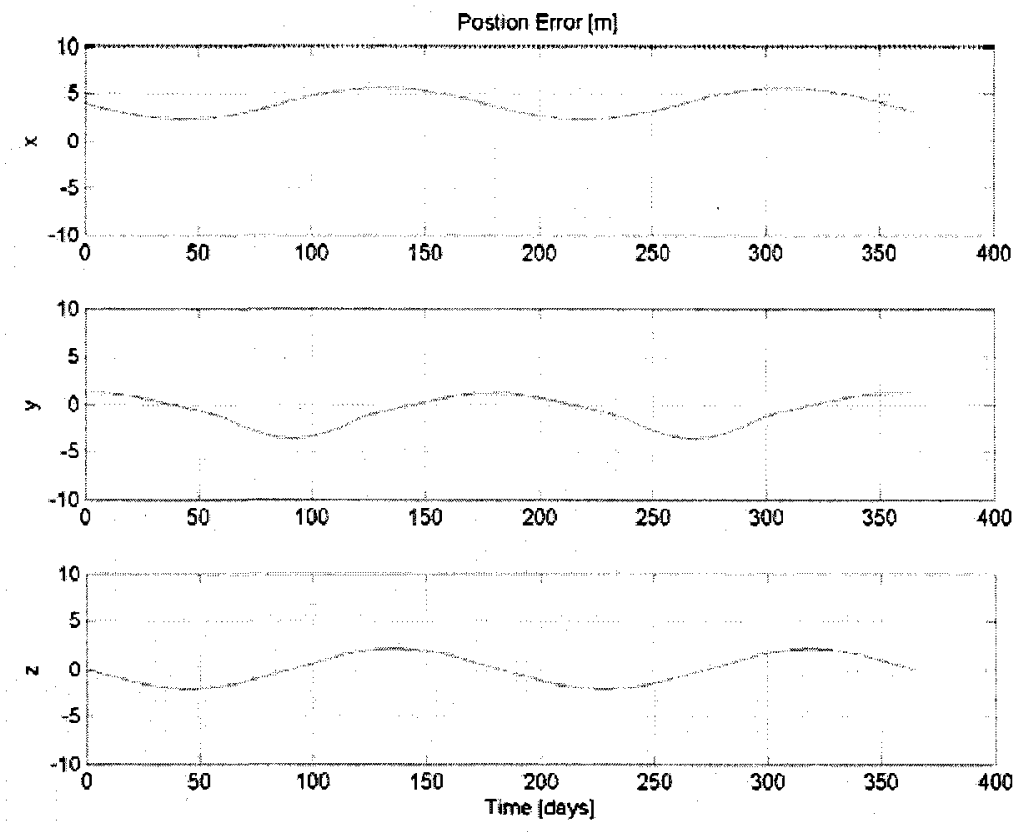

Figure 9.18 - SMC position errors for 1 year (Jupiter)
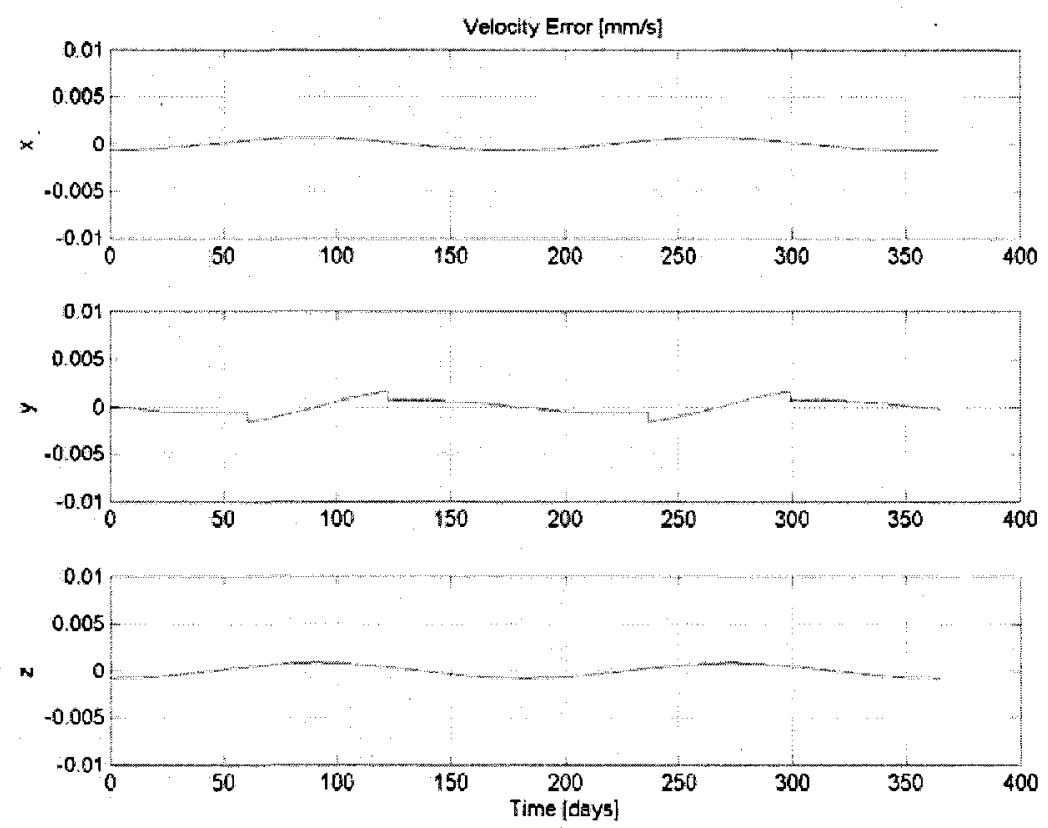

Figure 9.19 - SMC velocity errors for 1 year (Jupiter) 


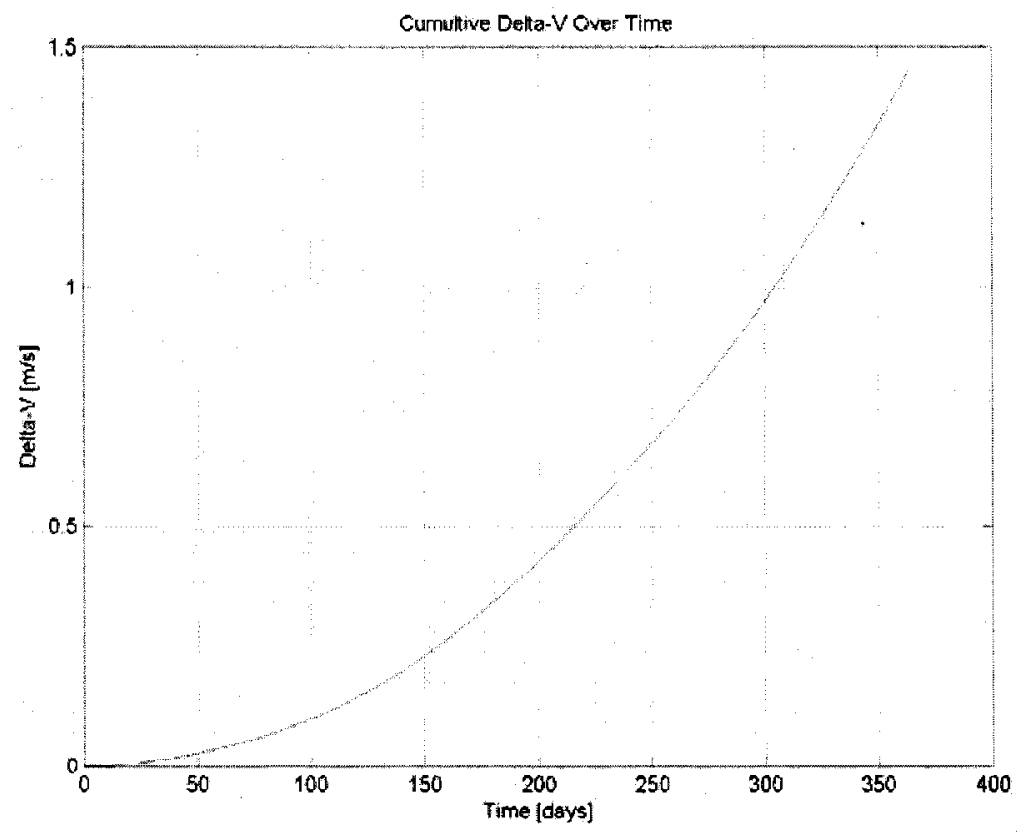

Figure 9.20 - SMC $\Delta \mathrm{v}$ profile for 1 year (Jupiter)

\begin{tabular}{|c|c|c|c|c|}
\hline & \multicolumn{3}{|c|}{ Maximum Errors } & \multirow{2}{*}{$\begin{array}{l}2 \mathrm{v} \\
\mathrm{m} / \mathrm{s})\end{array}$} \\
\hline & $x$ & $y$ & $z$ & \\
\hline position $[m]$ & 5.6 & 3.6 & 2 & \multirow{2}{*}{1.46} \\
\hline velocity $[\mu \mathrm{m} / \mathrm{s}]$ & 0.69 & 1.6 & 0.83 & \\
\hline
\end{tabular}

Table 9.5 - SMC results for 1 year (Jupiter)

Once again, the controller produces results equal to those of the unperturbed system. The SMC controller is shown to not be affected against gravitational forces of Jupiter. 


\subsection{Solar Radiation Pressure Results}

Results pertaining to the SMC controller applied to the nonlinear system with the presence of solar radiation pressure are shown in Figures 9.21 through 9.23. Numerical results are listed in Table 9.9.
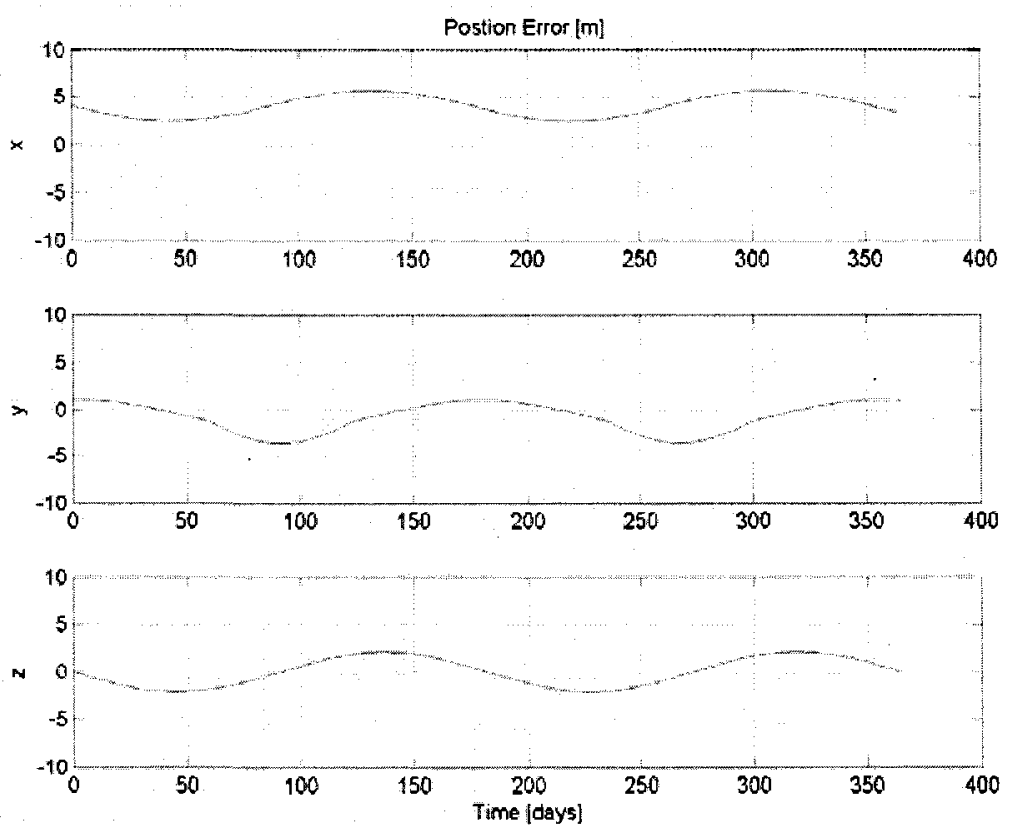

Figure 9.21 - SMC position errors for 1 year (solar radiation pressure) 

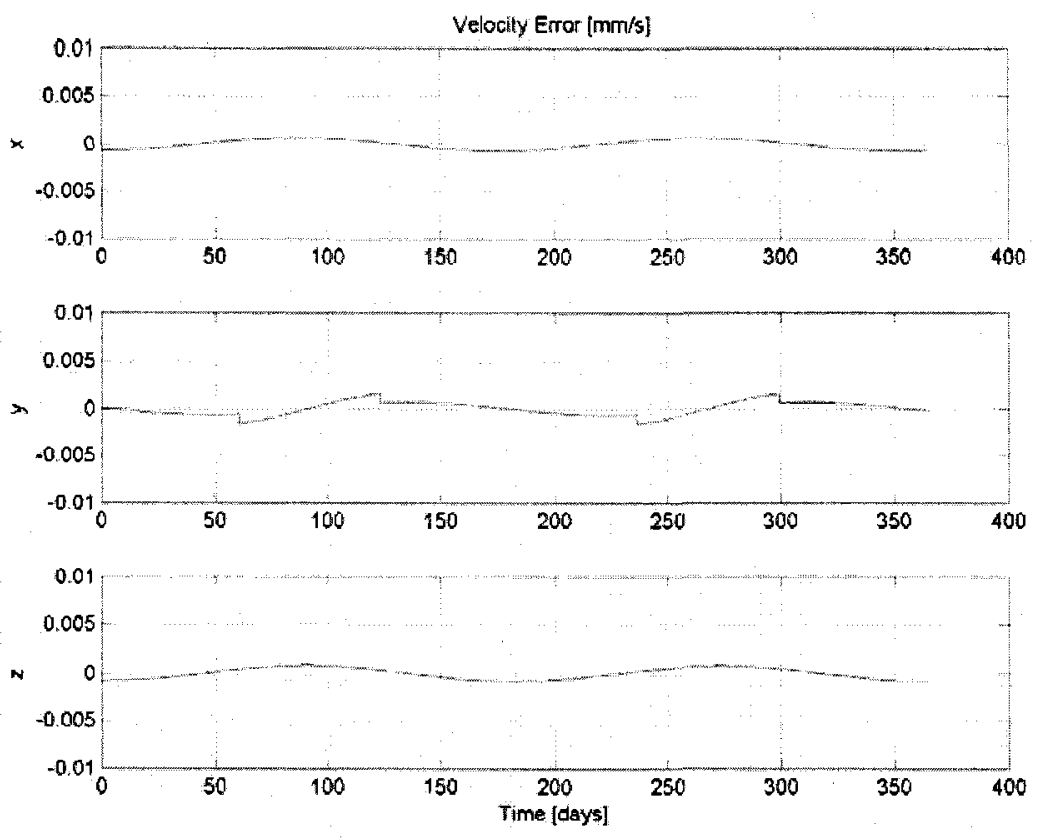

Figure 9.22 - SMC velocity errors for 1 year (solar radiation pressure)

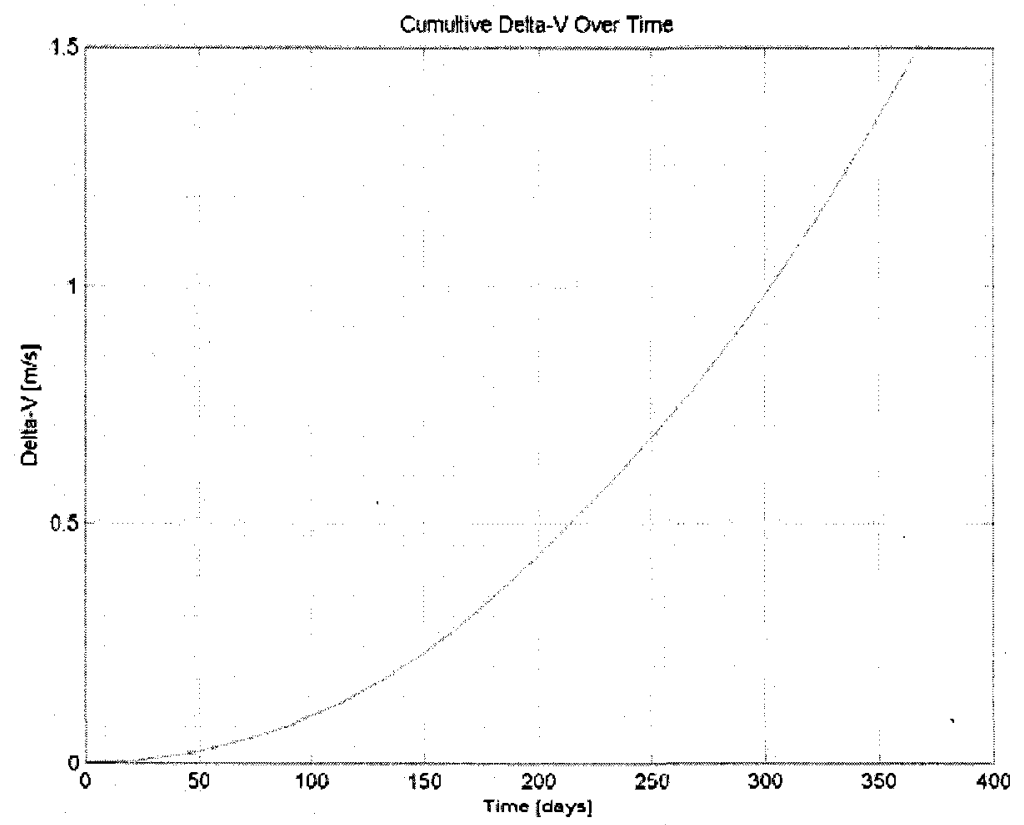

Figure 9.23 - SMC $\Delta v$ profile for 1 year (solar radiation pressure) 


\begin{tabular}{|c|c|c|c|c|}
\hline & \multicolumn{3}{|c|}{ Maximum Errors } & \multirow{2}{*}{ 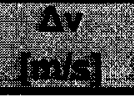 } \\
\hline & $x$ & y & 2 & \\
\hline position [m] & 5.7 & 3.6 & 2 & \multirow{2}{*}{1.48} \\
\hline velocity $[\mu \mathrm{m} / \mathrm{s}]$ & 0.69 & 1.6 & 8.3 & \\
\hline
\end{tabular}

Table 9.6 - SMC results for 1 year (solar radiation pressure)

Figures 9.17 through 9.19 shows the position errors are kept within the tolerance and the $\Delta \mathrm{v}$ is increased slightly, but still a small amount. The SMC controller is shown to provide satisfactory results against solar radiation pressure.

\subsection{Measurement Noise Results}

Results pertaining to the SMC controller applied to the nonlinear system with the presence of measurement noise are shown in Figures 9.24 through 9.26. Numerical results are listed in Table 9.9. 

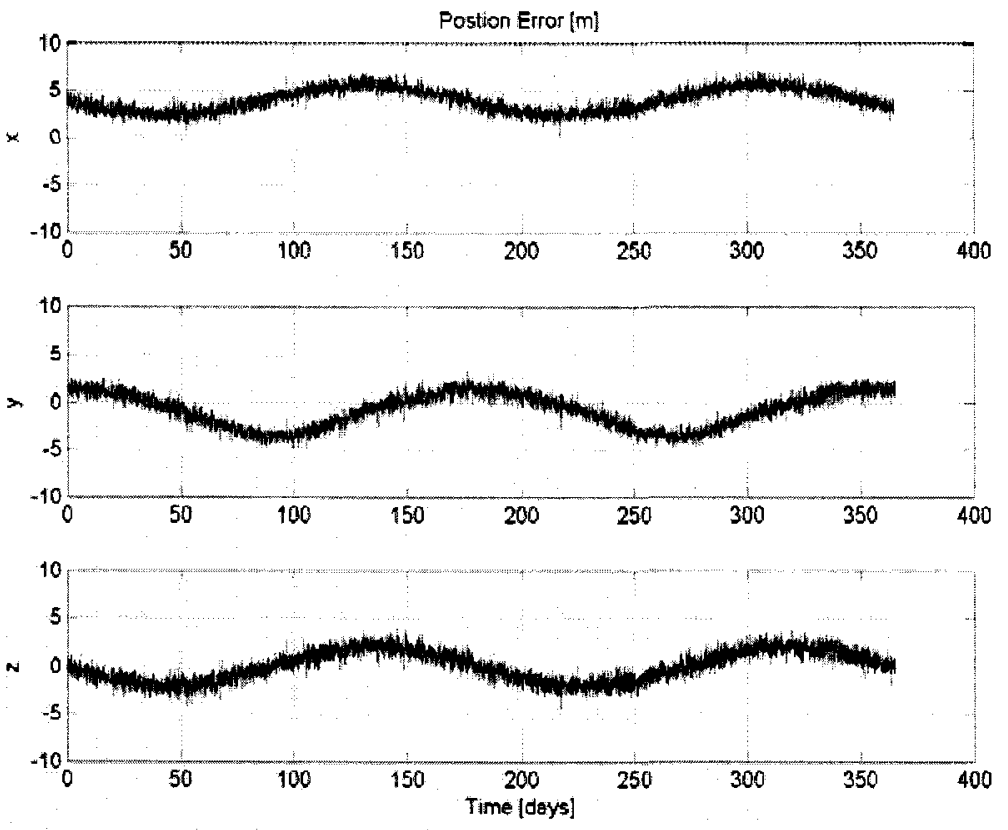

Figure 9.24 - SMC position errors for 1 year (measurement noise)
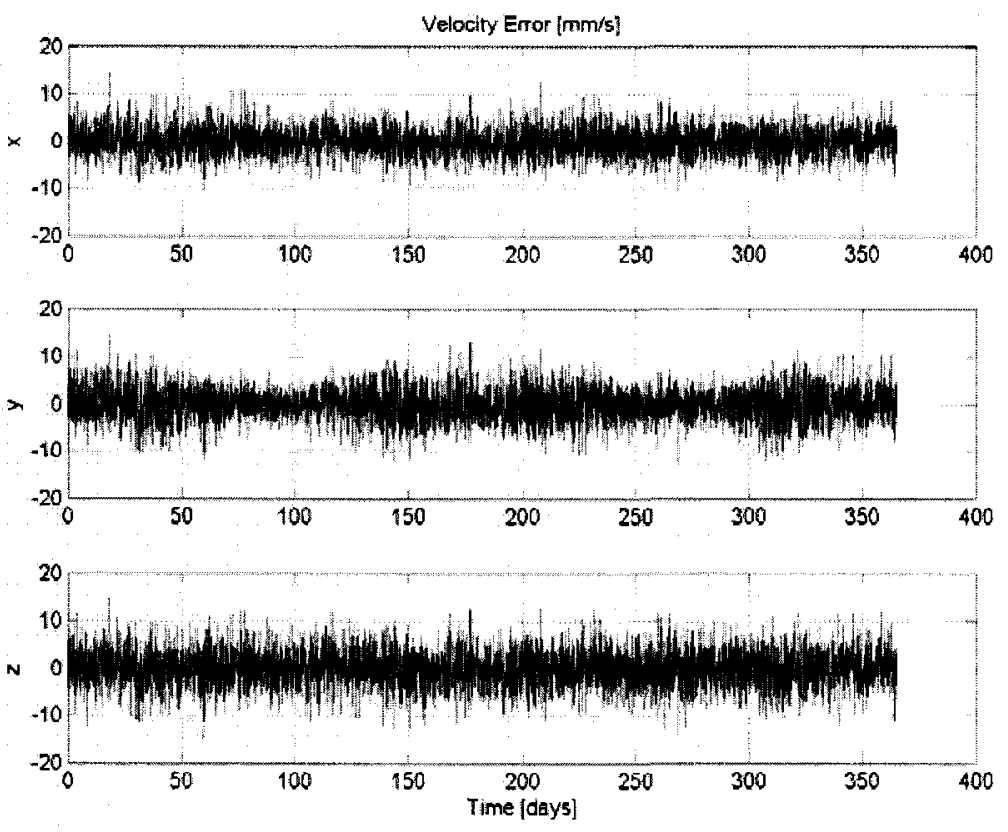

Figure 9.25 - SMC velocity errors for 1 year (measurement noise) 


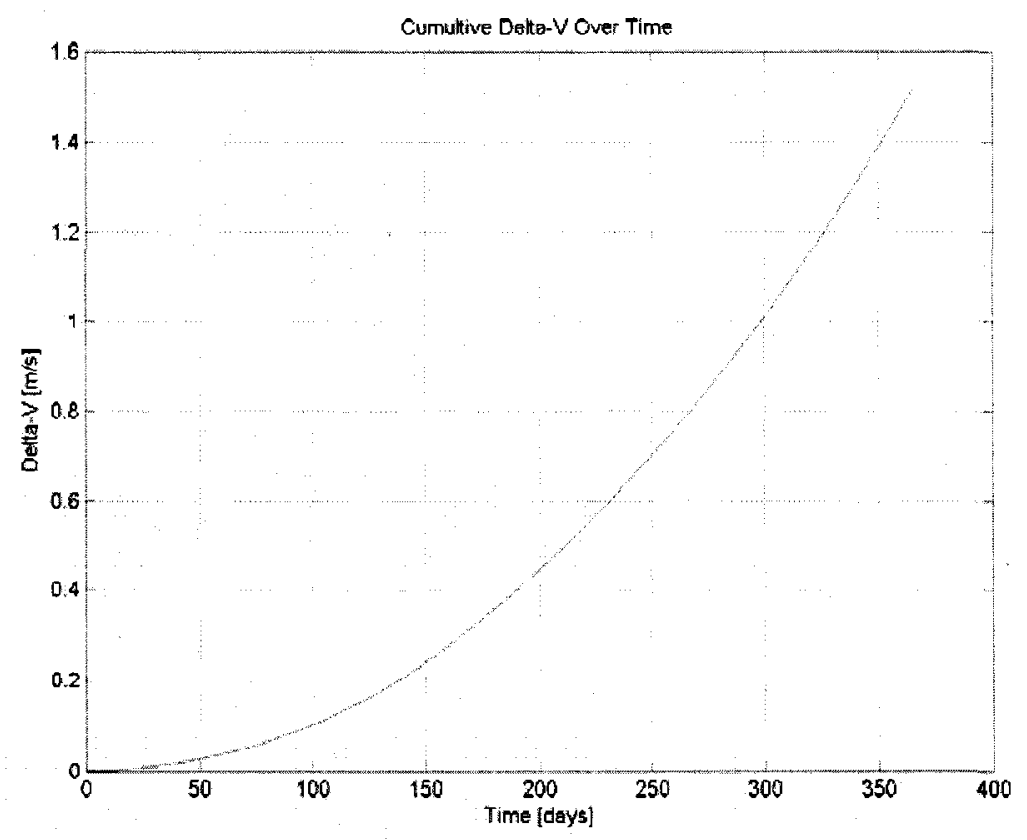

Figure 9.26 - SMC $\Delta v$ profile for 1 year (measurement noise)

\begin{tabular}{|c|c|c|c|c|}
\hline & \multicolumn{3}{|c|}{ Maximum Erors } & \multirow{2}{*}{$\begin{array}{l}4 \\
751\end{array}$} \\
\hline & $x$ & $y$ & $z$ & \\
\hline position [m] & 7.1 & 4.8 & 4.4 & \\
\hline velocify $[\mathrm{m} / \mathrm{s}]$ & 0.015 & 0.015 & 0.015 & \\
\hline
\end{tabular}

Table 9.7 - SMC results for 1 year (measurement noise)

The resulting position errors from the noisy measurement are noticeably higher, but are still within the allowable tolerance. The velocity errors, however, increased greatly. This is to be expected, though, since the unperturbed velocity errors were much less than the noise levels, yielding the noise to be seen as the primary error. Without a filter on the measurements, there is not much that can be done. However, since the mission requirements do not put any restrictions on velocity error, this may be considered as acceptable. Since the position errors are within the allowable requirements and $\Delta v$ is 
maintained at a minimum, the SMC controller is considered to perform adequately in the presence of the defined measurement noise..

\subsection{Differing Initial Conditions Results}

Results pertaining to the SMC controller applied to the nonlinear system with incorrect initial conditions are shown in Figures 9.27 through 9.29. Numerical results are listed in Table 9.8.
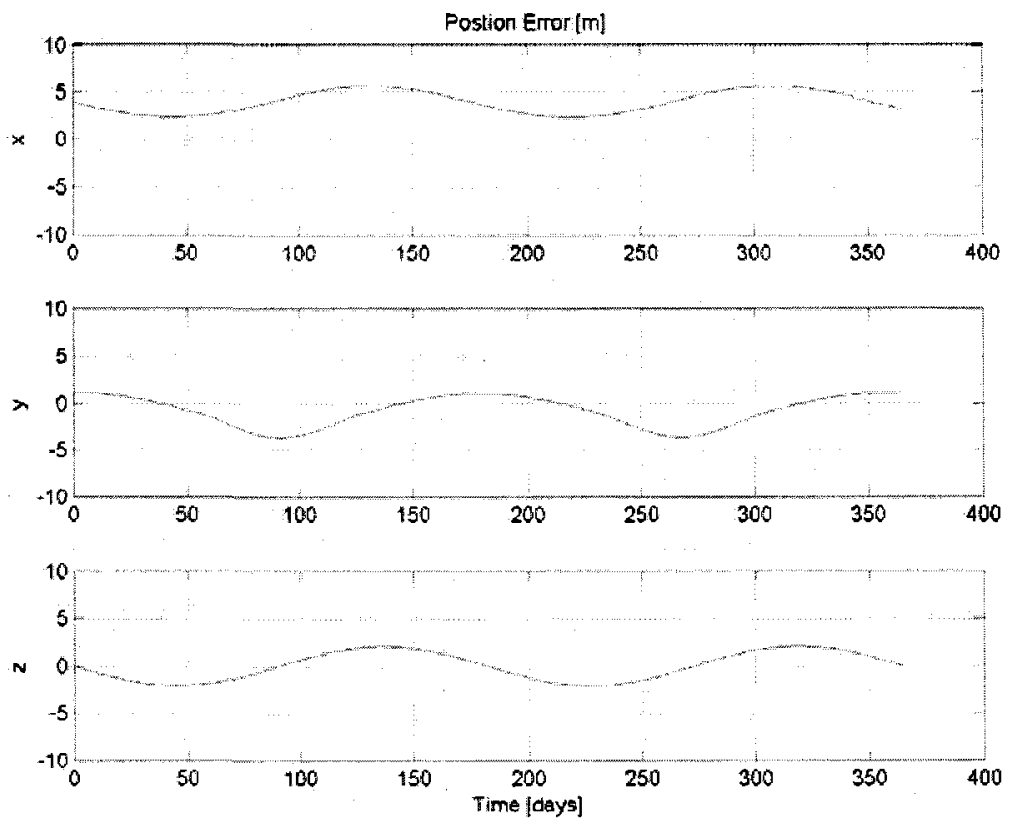

Figure 9.27 - SMC position errors for 1 year (differing initial conditions) 

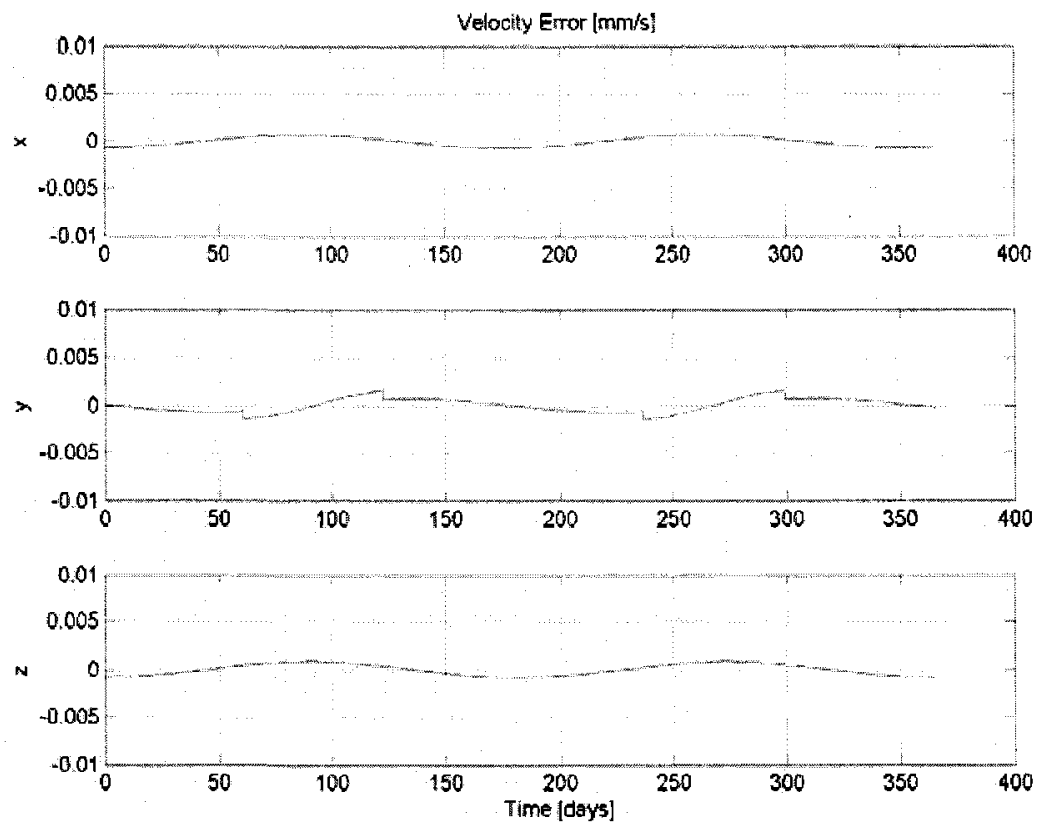

Figure 9.28 - SMC velocity errors for 1 year (differing initial conditions)

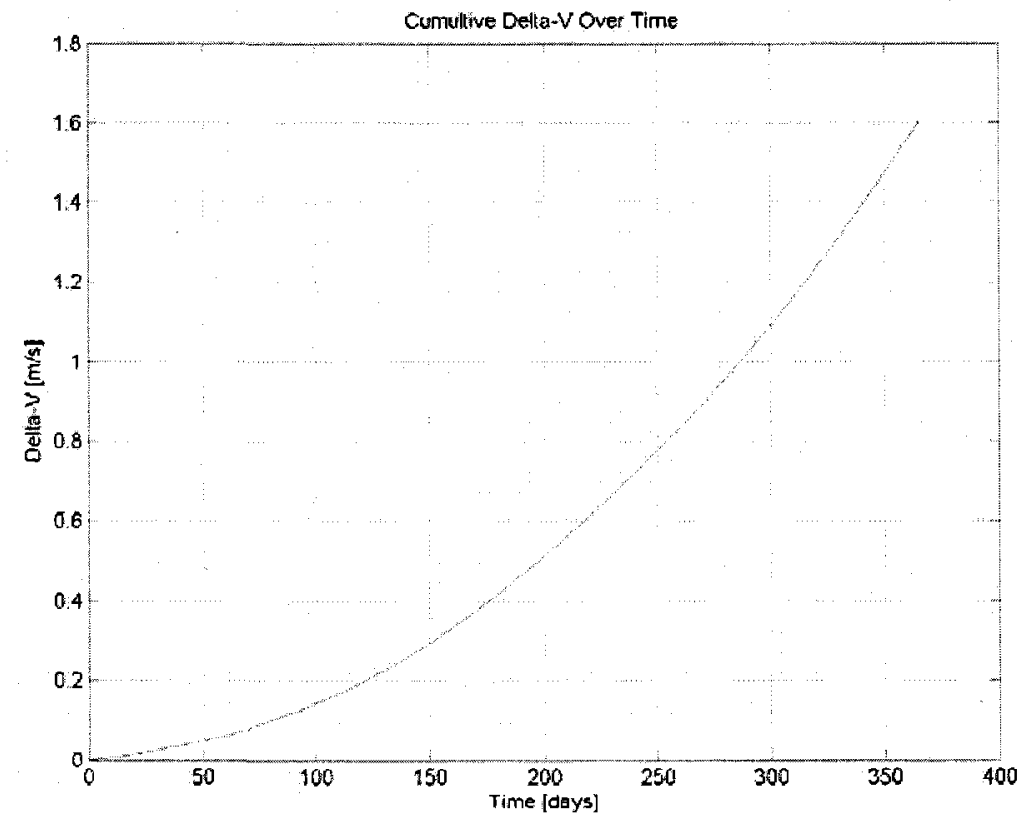

Figure 9.29 - SMC $\Delta \mathrm{v}$ profile for 1 year (differing initial conditions) 


\begin{tabular}{|c|c|c|c|c|}
\hline & \multicolumn{3}{|c|}{ Maximum Errors } & \multirow{2}{*}{$\begin{array}{l}2 y \\
\text { mo }\end{array}$} \\
\hline & $x$ & $y$ & 2 & \\
\hline postiontm] & 5.6 & 3.6 & 2.1 & \multirow{2}{*}{1.6} \\
\hline velocity $[\mathrm{Hm} / \mathrm{s}]$ & 0.69 & 1.6 & 8.3 & \\
\hline
\end{tabular}

Table 9.8 - SMC results for 1 year (differing initial conditions)

The SMC controller does well in maintaining errors within the mission allowance but allows the $\Delta \mathrm{v}$ to increase slightly. The control compensates for the large initial offset immediately but does not expend a large amount of initial effort as the previous controllers do. Instead, the $\Delta v$ rises to its linear profile quicker. Once the error is reduced, the $\Delta v$ returns to a minimum. This phenomenon can be seen in Figure 9.25 Although the $\Delta \mathrm{v}$ is increased compared to the unperturbed system, it is only increased by $0.05 \mathrm{~m} / \mathrm{s}$, concluding that $\mathrm{SMC}$ is robust against differing initial conditions.

\subsection{Worst Case Scenario Results}

Results pertaining to the SMC controller applied to the nonlinear system with the presence of all modeled disturbances and perturbations are shown in Figures 9.30 through 9.32. Numerical results are listed in Table 9.9. 

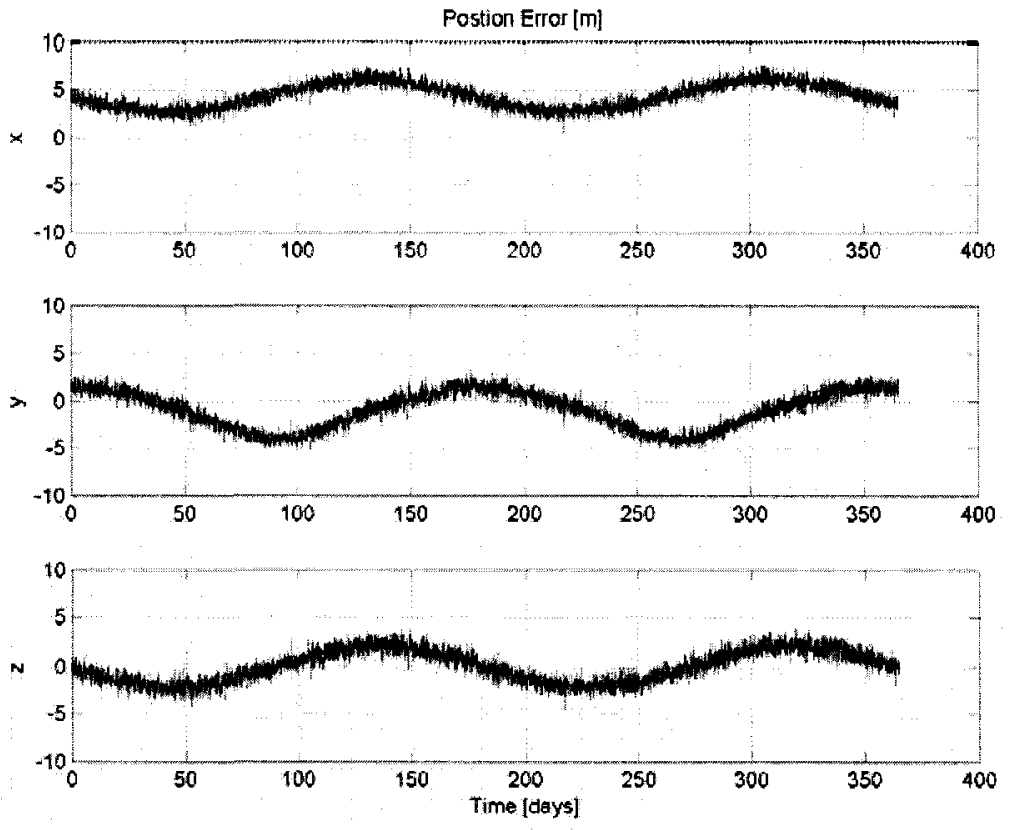

Figure 9.30 - SMC position errors for 1 year (worst case)
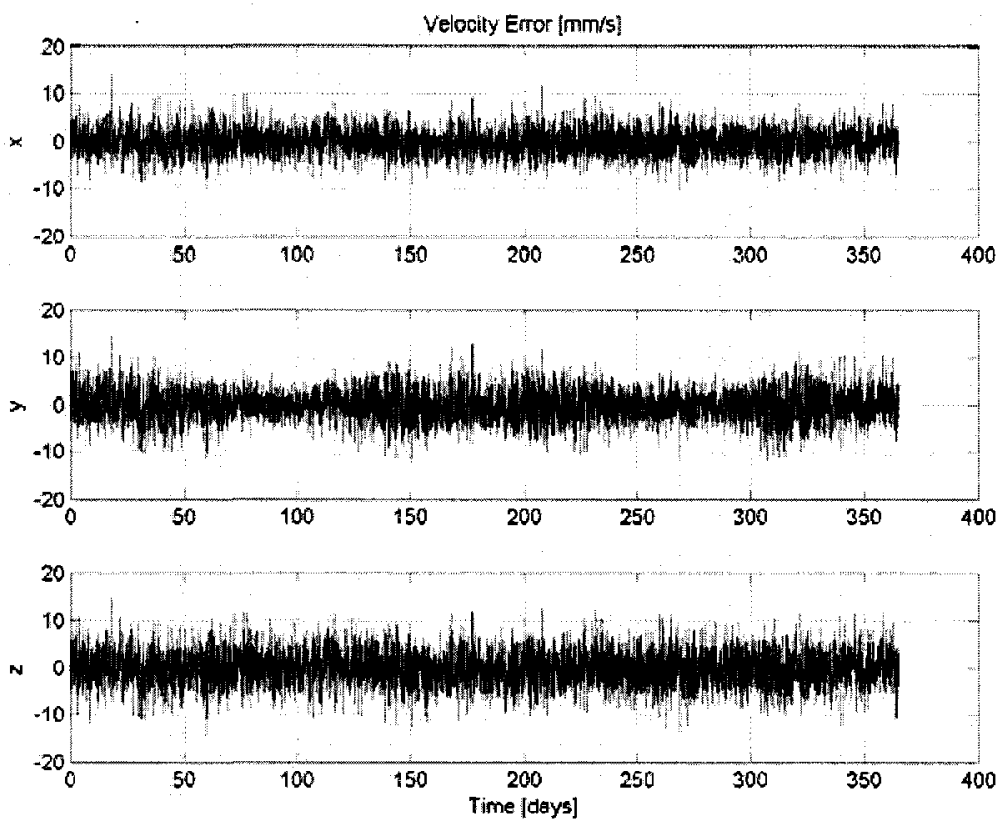

Figure 9.31 - SMC velocity errors for 1 year (worst case) 


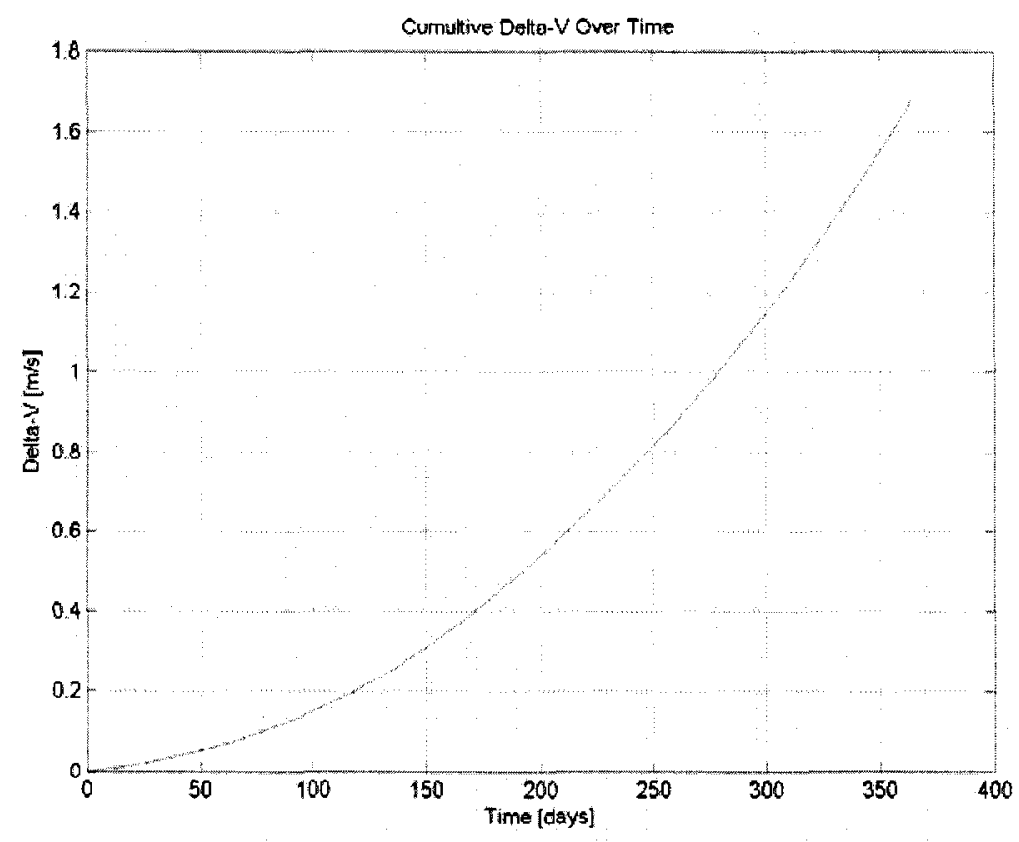

Figure 9.32 - SMC $\Delta v$.profile for 1 year (worst case)

\begin{tabular}{|c|c|c|c|c|}
\hline & \multicolumn{3}{|c|}{ Maximum Errors } & \multirow{2}{*}{ 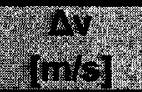 } \\
\hline & $\mathrm{x}$ & $y$ & $z$ & \\
\hline position [km] & 7.6 & 5.2 & 4.5 & \multirow{2}{*}{1.68} \\
\hline velocity $[\mathrm{m} / \mathrm{s}]$ & 0.014 & 0.015 & 0.015 & \\
\hline
\end{tabular}

Table 9.9 - SMC results for 1 year (worst case)

The results of the worst case scenario experience characteristics similar to the two cases with abnormal behavior, measurement noise and differing initial conditions. There exists high velocity errors and a faster exponential rise. However, even in the presence of all the disturbances and perturbations, the position errors are maintained within the tolerances and the $\Delta v$ is not significantly larger than the unperturbed system. The results yielded conclude that SMC control is robust enough to maintain stable even in this worst case scenario for the given mission and requirements. 


\subsection{Summary}

\begin{tabular}{|c|c|c|c|c|}
\cline { 2 - 5 } \multicolumn{1}{c|}{} & \multicolumn{3}{c|}{ Max Position Errors [ $]$} & [. \\
\cline { 2 - 5 } \multicolumn{1}{c|}{} & $\mathbf{x}$ & $\mathbf{y}$ & $\mathbf{z}$ & \\
\hline Unperturbed & 5.6 & 3.6 & 2 & 1.46 \\
\hline Thr. Misalign. & 5.6 & 3.6 & 2 & 1.46 \\
\hline Thr. Blas & 6 & 3.9 & 2.1 & 1.46 \\
\hline Self-Gravity & 5.6 & 3.6 & 2 & 1.46 \\
\hline Jupiter Gravity & 5.6 & 3.6 & 2 & 1.46 \\
\hline SRP & 5.7 & 3.6 & 2 & 1.48 \\
\hline Meas. Noise & 7.1 & 4.8 & 4.4 & 1.51 \\
\hline Differing IOs & 5.6 & 3.6 & 2.1 & 1.6 \\
\hline Worst Case & 7.6 & 5.2 & 4.5 & 1.68 \\
\hline
\end{tabular}

Table 9.10 - SMC results (summary)

Reviewing Table 9.10, SMC can be considered a robust controller for each of the cases investigated, producing errors within the tolerance and minimizing $\Delta \mathrm{v}$. Although the worst case scenario yields a $\Delta v$ higher than the unperturbed case, the increase is still within an acceptable range, this would be expected in the presence of all disturbances and perturbations. SMC is, therefore, a viable control method for the spacecraft mission with all classes of investigated perturbations. 


\section{CHAPTER 10}

\section{CONCLUSION}

\subsection{Comparison of Controllers}

\subsubsection{Unperturbed Results}

\begin{tabular}{|c|c|c|c|c|}
\cline { 2 - 5 } \multicolumn{1}{c|}{} & \multicolumn{3}{c|}{ Max Position Errors $[\mathbf{m}]$} & \\
\cline { 2 - 5 } \multicolumn{1}{c|}{} & $\mathbf{X}$ & $\mathbf{y}$ & $\mathbf{Z}$ & \\
\hline PID & 0.55 & 9.3 & 8.4 & 1.46 \\
\hline LQR & 7.2 & 6.1 & 5.9 & 1.46 \\
\hline $\boldsymbol{H}_{\infty}$ & 9.3 & 8.2 & 2.9 & 1.46 \\
\hline SMC & 5.6 & 3.6 & 2 & 1.46 \\
\hline
\end{tabular}

Table 10.1 - Unperturbed results (comparison)

Table 10.1 shows the results for each controller in an ideal state, without any disturbances or perturbations present. Each controller satisfies the mission requirements of maintaining errors within the allowable tolerance while concurrently minimizing $\Delta \mathrm{v}$. The control effort is equal for all controllers, thus position error takes priority, yielding SMC as the more efficient controller, maintaining the smallest overall error, although all controllers are viable methods.

\subsubsection{Thruster Errors and External Perturbations}

Thruster errors (misalignment, bias) and external perturbations (self-gravity, Jupiter, solar radiation pressure) do not affect the overall performance of the spacecraft for any of the 
controllers. Each controller maintains errors either equal to or similar to those presented in Table 10.1. Regardless, each controller displays the ability to sustain the same results, therefore SMC is still shows the best overall performance.

\subsubsection{Measurement Noise}

\begin{tabular}{|c|c|c|c|c|}
\hline & \multicolumn{3}{|c|}{ Max Position Errors $[m]$} & \multirow{2}{*}{ 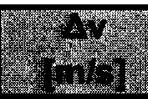 } \\
\hline & $x$ & $y$ & 2 & \\
\hline FiD & 48 & 17 & 15 & 36.4 \\
\hline LGR & 8 & 7.9 & 7.2 & 1.46 \\
\hline S $\mathrm{H}_{0}$ & 9.3 & 8.2 & 2.9 & 1.46 \\
\hline SMC & 7.1 & $\overline{4.8}$ & 4.4 & 1.51 \\
\hline
\end{tabular}

Table 10.2 - Measurement noise results (comparison)

Table 10.2 presents the results of the system with the introduction of measurement noise. It is obvious that PID can not perform as well under these conditions. As mentioned in Chapter 6, PID control is not designed to withstand measurement noise. The derivative gain corrupts the system by integrating a noisy signal, creating a nosier signal. The increase in position and velocities errors, therefore, increases the required $\Delta v$ to maintain stability. It can be concluded that PID is definitely not suitable for this formation flying mission. LQR and $\mathrm{H}_{\infty}$, however, perform just as well in this case as in the previous cases, maintaining the same errors and $\Delta \mathrm{v}$.

SMC, maintaining its errors, slightly increases it's $\Delta \mathrm{v}$. This can be attributed to the design decision of using LQR as the equivalent control. As previously stated, LQR is robust enough to withstand the disturbance. The introduction of the sliding control term adds extra, unnecessary control to a system that is already stable. So even though SMC 
maintains overall smaller errors, it requires a higher control effort, concluding that LQR is the more efficient control technique for this scenario.

\subsubsection{Differing Initial Conditions Results}

\begin{tabular}{|c|c|c|c|c|}
\cline { 2 - 5 } \multicolumn{1}{c|}{} & \multicolumn{3}{c|}{ Max Position Errors $[\mathbf{m}]$} & \\
\cline { 2 - 5 } & $\mathbf{x}$ & $\mathbf{y}$ & $\mathbf{z}$ & \\
\hline PID & 0.56 & 9.3 & 8.4 & 17.2 \\
\hline LQR & 7.2 & 6.1 & 5.9 & 1.51 \\
\hline$H_{\infty}$ & 4222 & 5653 & 7595 & 1.95 \\
\hline SMC & 5.6 & 3.6 & 2.1 & 1.6 \\
\hline
\end{tabular}

Table 10.3 - Differing initial conditions results (comparison)

Table 10.3 shows that each controller yields different results than previous cases, showing that each controller is affected by differing initial conditions. Once again PID shows a poor performance because of its inherent disadvantages. The controller is able to maintain the errors, although it requires a much higher control effort when beginning with differing initial conditions. During the transient phase, the controller exerts a great amount of $\Delta \mathrm{v}$ to recover from the large initial error. Once the errors are returned to "normal" values, the control effort is decreased. This proves that if a large error occurs while the spacecraft is in orbit, the controller would expend a great deal of energy to return to its correct trajectory.

$\mathrm{H}_{\infty}$ also suffers severely from differing initial conditions. As mentioned in Chapter 8, the controller is not designed to correct for large initial errors. The two-point boundary problem does not offer a disturbance model that adjusts tracking error for significantly differing initial conditions. Therefore, the spacecraft is never able to recover from the 
initial five kilometer error. As the PID control, that $\mathrm{H}_{\infty}$ would not be able to recover from a large unexpected error in mid-orbit.

LQR and SMC maintain reasonable results as shown in previous sections, albeit slightly higher position and velocity error and $\Delta \mathrm{v}$. As with the measurement noise, however, SMC exerts an extra, unnecessary control, to maintain smaller errors, but with a slightly higher $\Delta \mathrm{v}$. Here LQR may be considered to be the more efficient control technique if fuel economy is weighted over position accuracy.

\subsubsection{Overall Performance}

Out of the control methods studied, the only two controllers that are able to maintain reasonable results with the scenarios investigated are $L Q R$ and SMC. These two controllers produce very similar results, with SMC errors slightly smaller than LQR. In the presence of extreme disturbances and perturbations, SMC begins to produce unnecessary control effort. Here, the simpler control technique of LQR, slightly outperforms the more complicated SMC, yielding the claim that LQR is the more efficient controller for the cases investigated in this study, if one is not as concerned with minimizing accuracy. 


\subsection{Future Work}

Although the LQR control technique appears to be the more efficient technique for this specific mission, according to these results, there is much more research that can be done:

- Other control techniques should be tested, including those omitted in the begin of this research, such as feedback linearization, adaptive control and gain scheduling. A more efficient option may be available.

- More disturbances must be examined. As discussed earlier, there is a plethora of disturbances that exist in the space environment that could prove to be significant. Gravitational forces from the Moon and the eccentricity of the Earth are two main perturbations that are of interest. [17] provides a large analysis disturbances present in the $\mathrm{L}_{2}$ region. Most or all of them should be analyzed.

- Test the system for other orbit types and locations, such as a halo orbit or an orbit positioned off of $\mathrm{L}_{2}$.

- Discretize the system and controllers. When in orbit, the spacecraft will not be able to receive continuous orbital feedback. There are usually delays on the order of days between each sampling period.

- Model thrusters more accurately. The thrusters in this study assume analog and limitless output. In actuality, thrusters are quantized "on" or "off" and cannot output analog thrust.

- Lastly, the orbital control system should be integrated with an attitude control system, taking into account the orientation of the spacecraft throughout the entire 
mission. The spacecraft will need to have the correct orientation to send/receive signals and to perform appropriate orbital maneuvers.

\section{$\underline{10.3 \text { Final Remarks }}$}

Throughout this study several controllers were investigated and tested against selected disturbance and perturbations that a spacecraft formation might exhibit while flying about the Earth/Moon-Sun $L_{2}$ libration point. Each of the four control techniques were tested against these disturbances to discover if there exists the possibility of maintaining small errors while minimizing control effort. The results showed that out of the four controllers, two (PID and $\mathrm{H}_{\infty}$ ) were not robust enough to reject disturbances. These two controllers either exceeded the allowable error tolerance or severely increased fuel usage.

The remaining two controllers, LQR and SMC, both maintained errors well within the allowable error tolerance and control efforts of each of these methods remained small despite the presence of various disturbances and perturbations. The difference between the two is that SMC was seen to produce a small amount of unneeded control effort to reduce the tracking error to slightly smaller amounts than those recorded by LQR. In terms of the mission requirements of maintaining errors within a ten meter tolerance while minimizing fuel use, LQR is seen as the more efficient of the two controllers. However, if one were to become more concerned with system accuracy, while leaving some allowance on fuel usage, SMC would be the wiser choice of control. 
To summarize, out of the control techniques investigated, $L Q R$ and $\mathrm{SMC}$ are able to satisfy mission requirements, but the final choice of control technique is directly related to tradeoff between accuracy and the desire to decrease fuel consumption. 


\section{BIBLIOGRAPHY}

[1] B. Wie. Space Vehicle Dynamics and Control. American Institute of Aeronautics and Astronautics, 1998.

[2] A.M. Segerman and M. F. Zedd. "Investigations of Spacecraft Orbits Around the $L_{2}$ Sun-earth Libration Point”. Naval Research Laboratory, Washington, DC. 2004

[3] A. Sinha. Linear Systems: Optimal and Robust Control. Taylor \& Francis, 2007.

[4] G. F. Franklin, J. D. Powell and A. Emami-Naeini. Feedback Control of Dynamic Systems. Pearșons Education, 2002.

[5] M. Green and D. J. N. Limebeer. Linear Robust Control. Prentice-Hall, 1995.

[6] J. J. E. Slotine and W. Li. Applied Nonlinear Control. Prentice-Hall, 1991.

[7] C. Edwards and S. K. Spurgeon. Sliding Mode Control: Theory and Applications. Taylor \& Francis, 1998.

[8] Solar Terrestrial Relations Observatory (STEREO) Project Website. http://www.stereo.jhuapl.edu/

[9] D. Folta, K. Hartman, K. Howell, and B. Marchand. "Formation Control of the MAXIM $\mathrm{L}_{2}$ Libration Orbit Mission". In American Institute of Aeronautics and Astronautics, pages 1-18, 2004.

[10] BNSC - Formation Flying Website. http://www.bnsc.gov.uk/content.aspx?nid=6068.

[11] Cluster Project

Website. http:/clusterlaunch.esa.int/science-e/www/area/index.cfm?fareaid $=8$ 
[12] Terrestrial Planet $\quad$ Finder $\quad$ (TPF) Project $\quad$ Website. http:/planetquest.jpl.nasa.gov/TPF/tpf index.cfm

[13] Gravity Recovery and Interior Laboratory (GRAIL) Project Website. http://sse.jpl.nasa.gov/missions/profile.cfm?Sort=Target\&Target=Moon\&MCode=GRAI L.

[14] B. G. Marchand. Spacecraft Formation Keeping Near the Libration Points of the SunEarth/Moon System. Phd Thesis, Perdue University, 2004.

[15] D. J. Scheeres, F.-Y. Hsiao and N. X. Vinh. "Stabilizing Motion Relative to an Unstable Orbit: Applications to Spacecraft Formation Flight". Journal of Guidance, Control, and Dynamics, 26(1):62-73, 2003.

[16] B. Marchand, K. C. Howell and R. S. Wilson. "An Improved Corrections Process for Constrained Trajectory Design in the n-Body Problem". Journal of Spacecraft and Rockets, 44(4):884-897, 2007.

[17] S. W. Evans. "Natural Environment Near the Sun/Earth-Moon L2 Libration Point". Marshall Space Flight Center, Alabama, 2003.

[18] M. H. Kaplan. Modern Spacecraft Dynamics \& Control. John Wiley and Sons, 1976.

[19] J. R. Wertz. Spacecraft Attitude Determination and Control. D. Reidel Publishing Company, 2002.

[20] T. Logsdon. Orbital Mechanics: Theory and Applications. John Wiley and Sons, 1998.

[21] M.W. Lo. "Libration point trajectory design". Numerical Algorithms, 14(1-3):153-164, 1997. 
[22] B. Wong, A. Misra. "Dynamics of a libration point multitethered system". International Astronatutical Federation - 55th International Astronautical Congress 2004, pages 457-467, 2004.

[23] D. W. Dunham, C. E. Roberts. "Stationkeeping techniques for libration-point satellites". Journal of the Astronautical Sciences, 49(1):127-144, 2001.

[24] Y. Xu, S. Suman. "Robust stationkeeping for libration point orbits". Spaceflight Mechanics 2006 (Advances in the Astronatical Science), 124(1):931-950, 2006.

[25] S. Berge, B. Jakobsson, P. Bodin, A. Edfors, S. Persson. "Rendezvous and formation flying experiments within the prisma in-orbit testbed". Proceedings of the $6^{\text {th }}$ International ESA Conference on Guidance, Navigation and Control Systems, page 10, 2006.

[26] J. Li, X. Meng, Y. Gao, X. Li. "Study on relative orbital configuration in satellite formation flying”. Acta Mechnica Sinica/Lixue Xuebao, 21(1):87-94, 2005.

[27] O. P. Lay, G. H, Blackwood. "Formation-flying interferometry." Proceedings of the SPIE - The International Society for Optical Engineering, 4852:481-491, 2003.

[28] S. I. Infield, S.B. Josselyn, W. Murray, I. M. Ross. "Design and control of libration point spacecraft formations". Journal of Guidance, Control, and Dynamics, 30(4):899$909,2007$.

[29] S.R. Vadali, H.-W. Bae, K. T. Alfriend. "Design and control of libration satellite formations". Advances in the Astronautical Sciences, 119(1):897-912, 2005.

[30] Magnetospheric Multicale (MMS) Mission Project Website. http://stp.gsfc.nasa.gov/missions/mms/mms.html 
APPENDICES 


\section{APPENDIX A}

\section{CR3BP AND DISTURBANCE MODELS}

The following appendices provide the necessary figures and coding, while limiting redundancy of figures and MATLAB code. The first appendix provides figures and code that all controllers use, while the following appendices provide a more-in depth look at the individual controllers.

\section{A.1 System SIMULINK Model}

Figures $\mathrm{A} 1$ and $\mathrm{A} 2$ show the overall system model. Each individual disturbance can be enabled or disabled withing from the $\mathrm{m}$-file. 


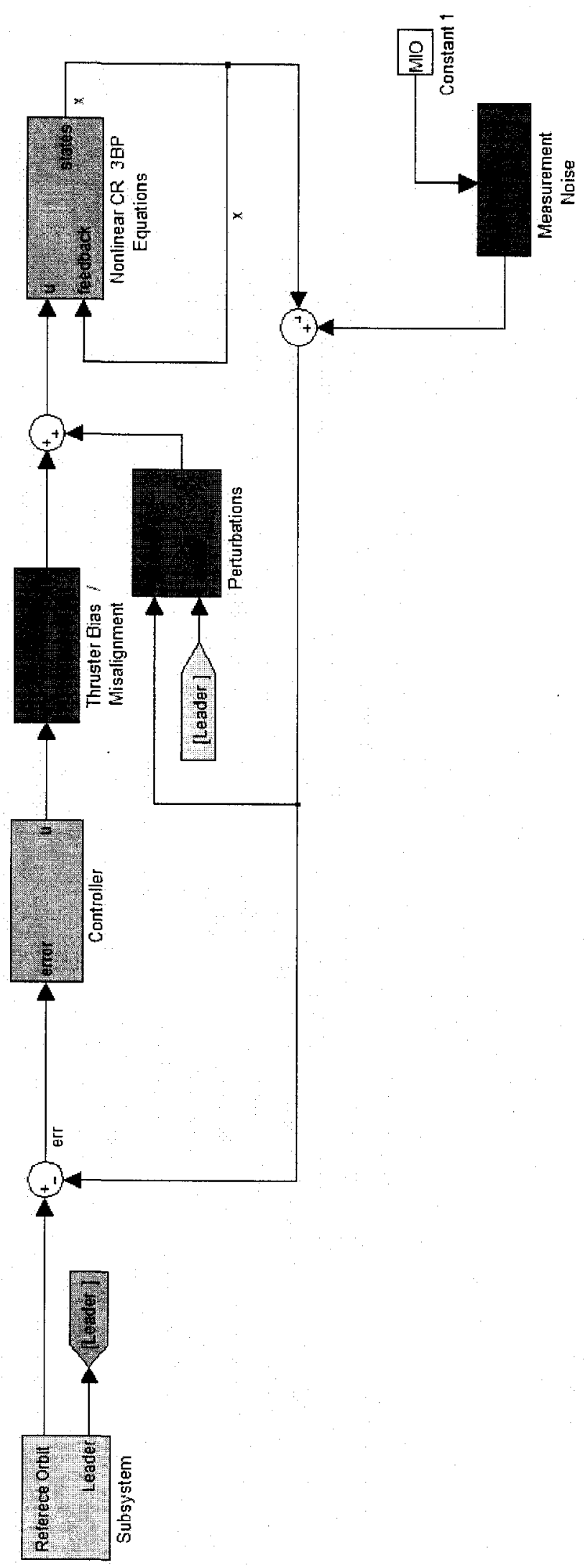

Figure A1 - Overall system model 

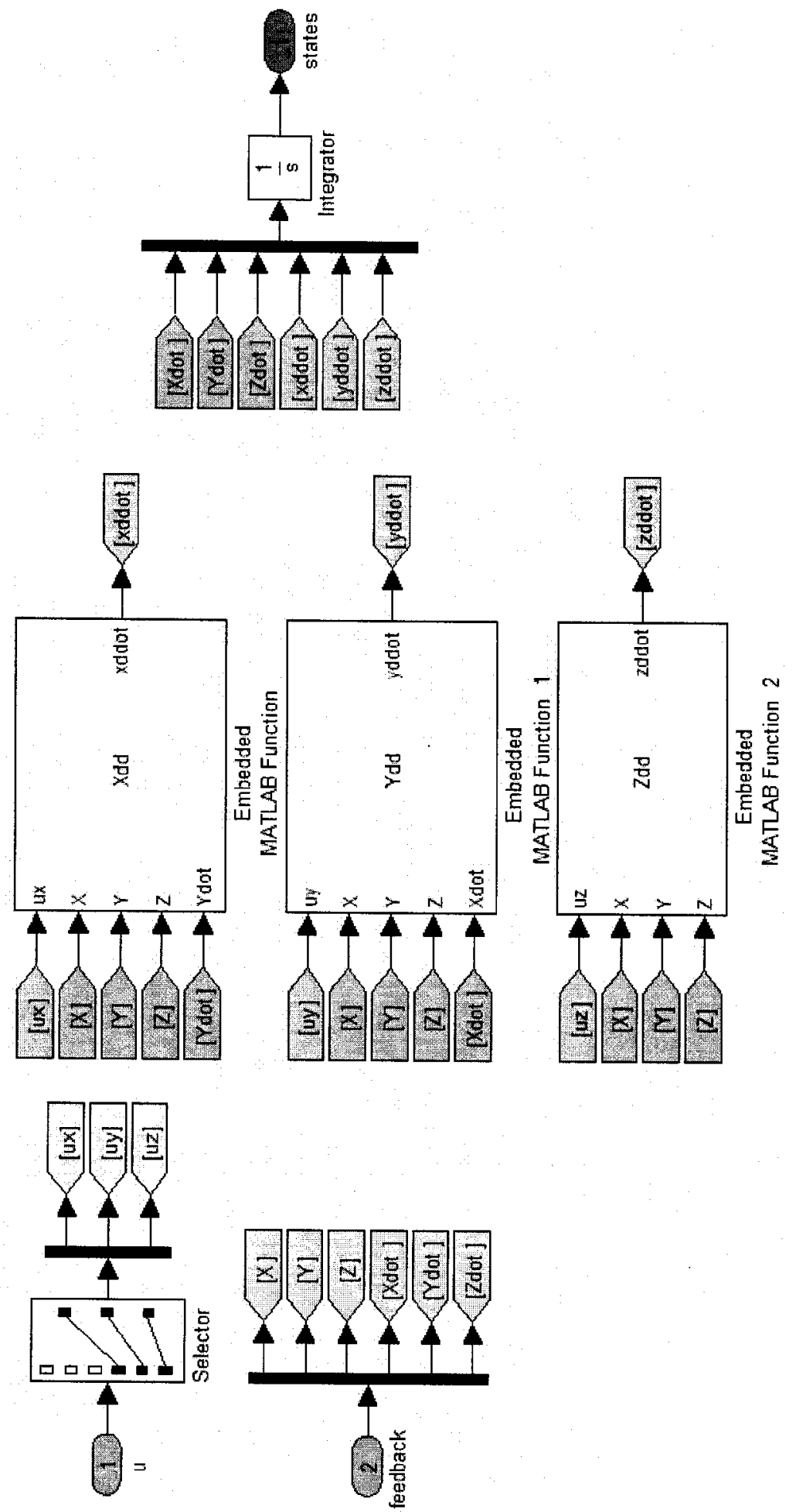

Figure A2 - Nonlinear CR3BP equations of motion 


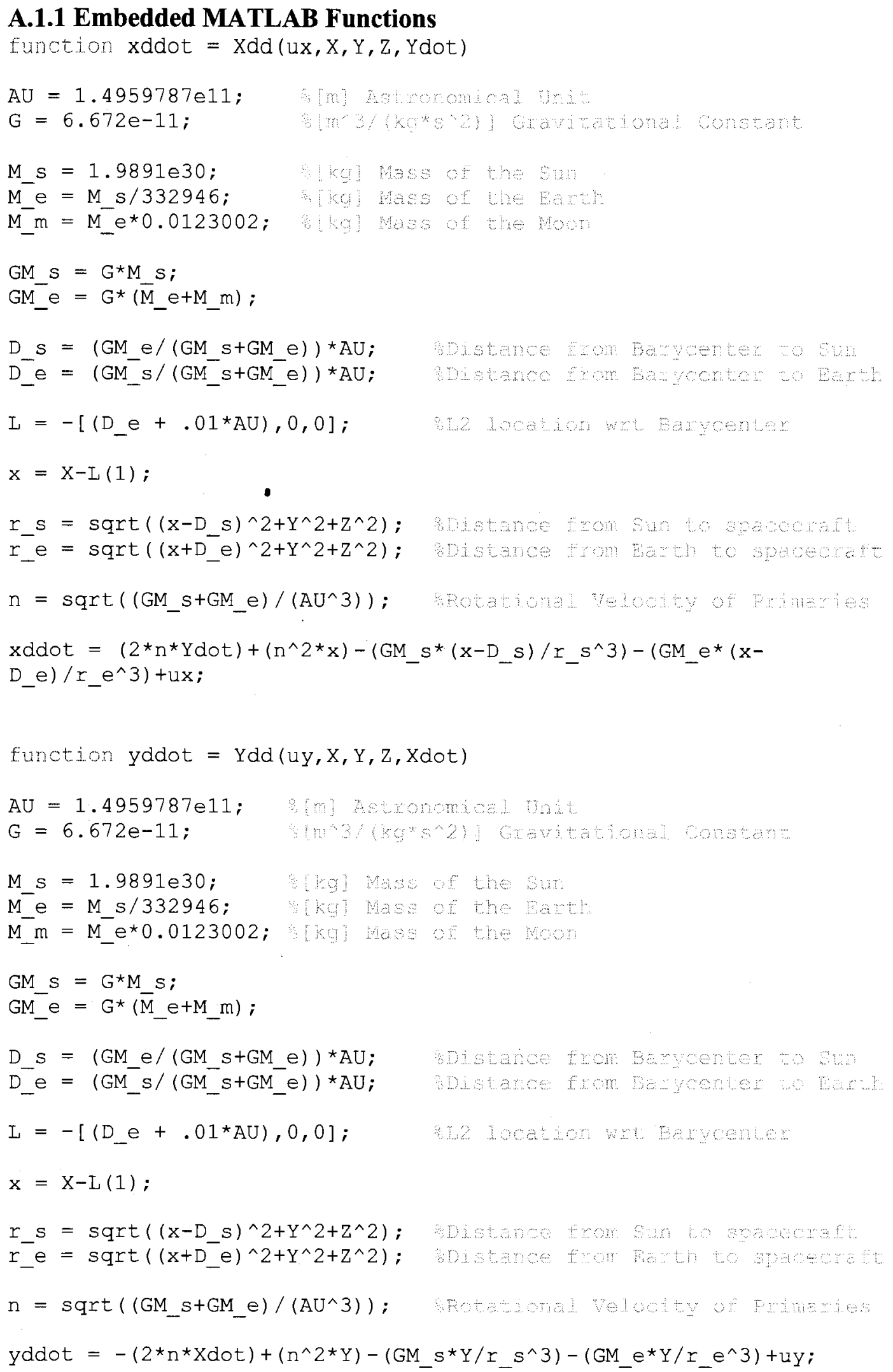


function $z d d o t=Z d d(u z, X, Y, Z)$

$\mathrm{AU}=1.4959787 \mathrm{e} 11 ;$

$\mathrm{G}=6.672 \mathrm{e}-11$;

me hemonom cos trit

M_s $=1.9891 \mathrm{e} 30 ; \quad$ (kg) Mase of the Sum

$M^{-} e=M_{-} / 332946$;

$\mathrm{M}^{-} \mathrm{m}=\mathrm{M}^{-} \mathrm{e}^{*} 0.0123002 ; \mathrm{kn} \mathrm{Hase}$ of the Mon

$\mathrm{GM} s=\mathrm{G}^{\star} \mathrm{M} s$;

GM_e $=G^{\star}\left(\bar{M} \_e+M \_m\right)$;

$D s=(G M e /(G M s+G M$ e $)) * A U$

$\mathrm{D}_{-}^{-} \mathrm{e}=\left(\mathrm{GM}_{-}^{-} \mathrm{s} /\left(\mathrm{GM}_{-}^{-} \mathrm{s}+\mathrm{GM}_{-}^{-} \mathrm{e}\right)\right) * \mathrm{AU}$

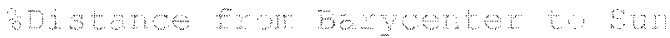

$L=-\left[\left(D_{-} e+.01 * A U\right), 0,0\right]$

$\mathrm{X}=\mathrm{X}-\mathrm{L}(1)$

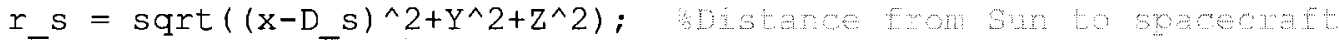

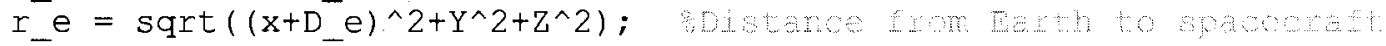

$z$ ddot $=-\left(G M \_s * z / r_{-} s^{\wedge} 3\right)-\left(G M \_e^{*} Z / r_{-} e^{\wedge} 3\right)+u z$

\section{A.2 Reference SIMULINK Model}

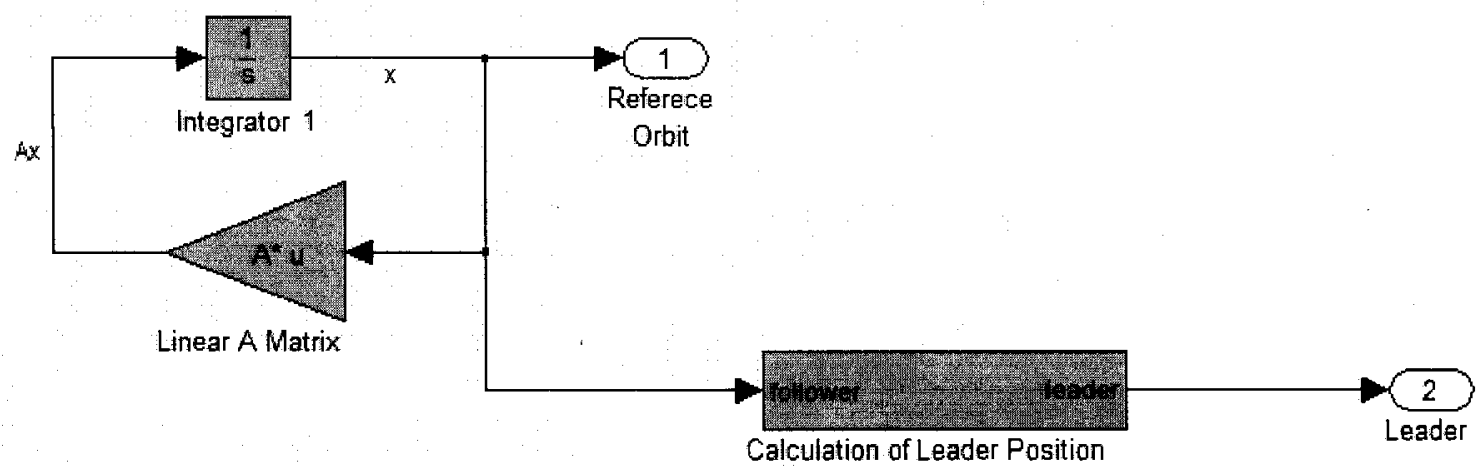

Figure A3 - Reference model 


\section{A.3 Disturbances}

\section{A.3.1 Thruster Errors}

Figures A4 through A10 will show the Simulink Models for all the disturbances.

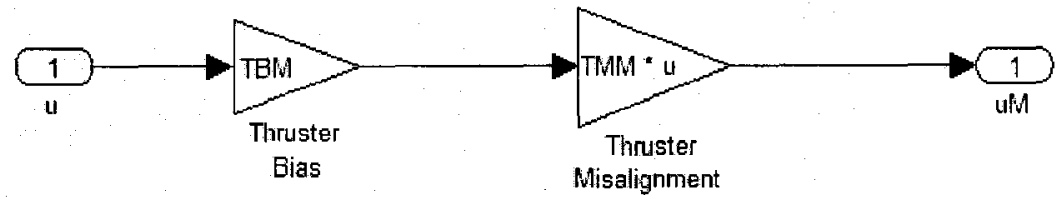

Figure A4 - Thruster bias / misalignment

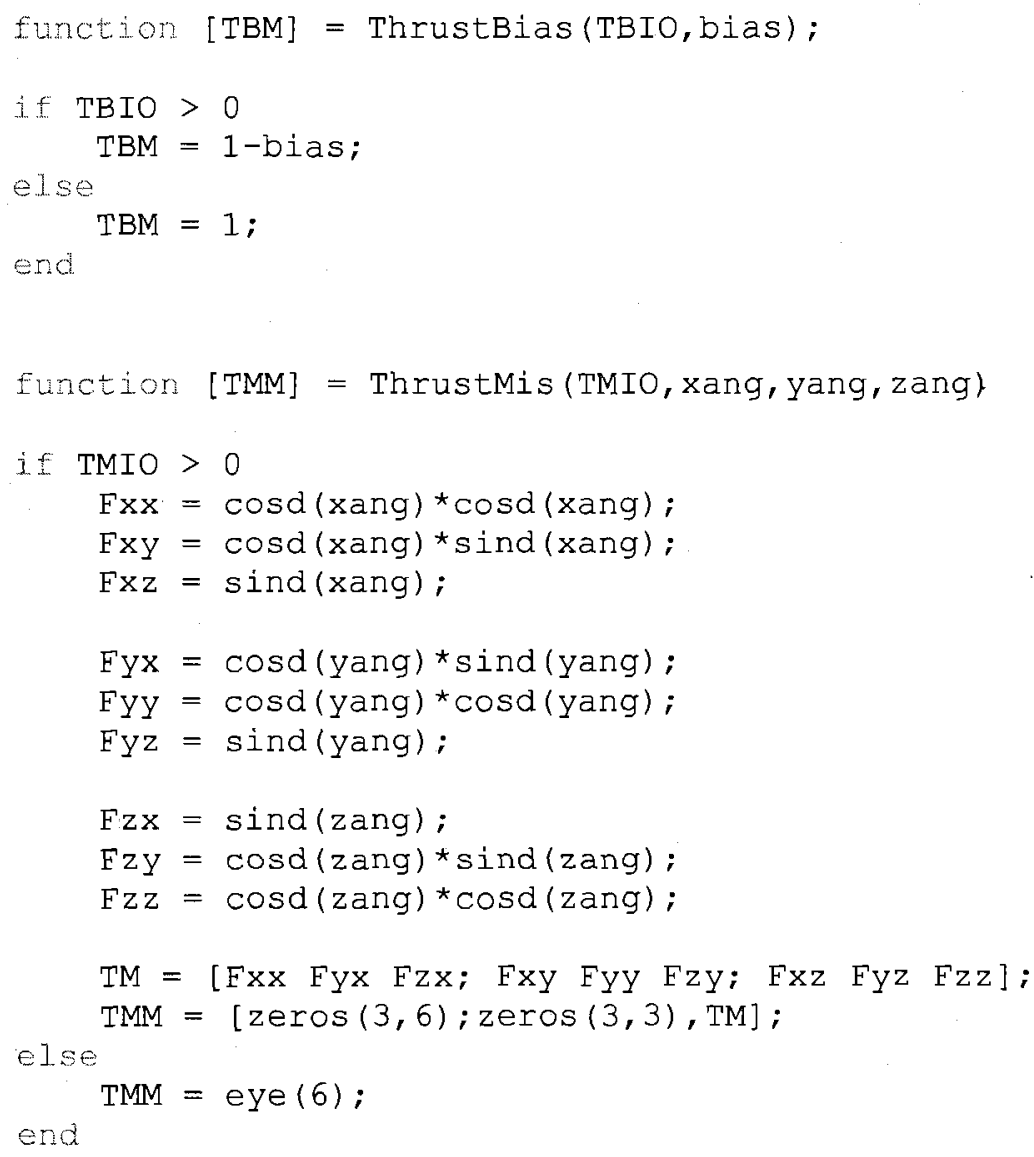

TBIO and TMIO are on/off switches for the disturbances, while the other inputs are the thruster bias and misalignment angles. 


\section{A.3.2 External Perturbations}

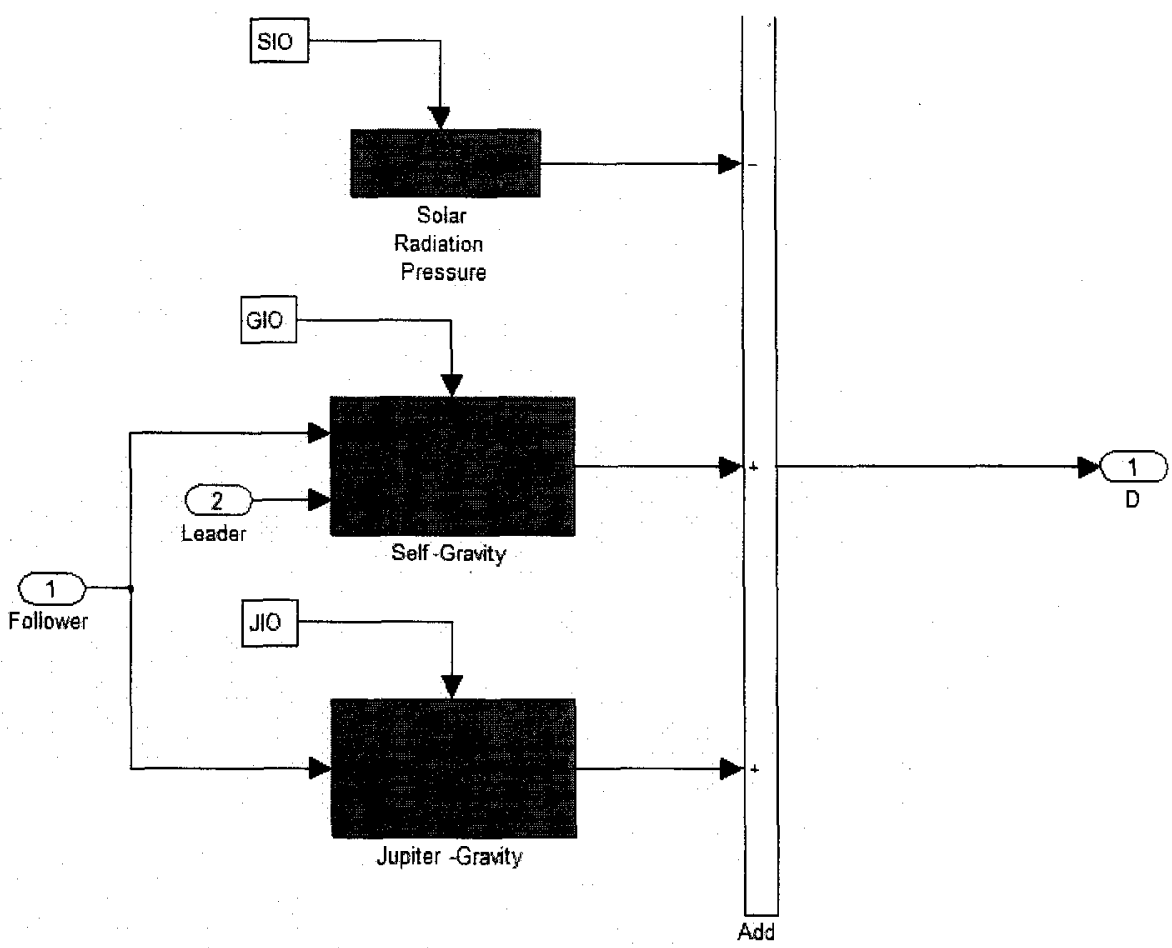

Figure A5 - Perturbations

All IO's are disturbance on/off switches

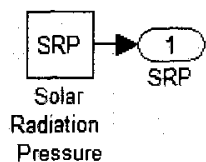

$$
\frac{\pi}{\text { Enable }}
$$

Figure A6 - Solar radiation pressure

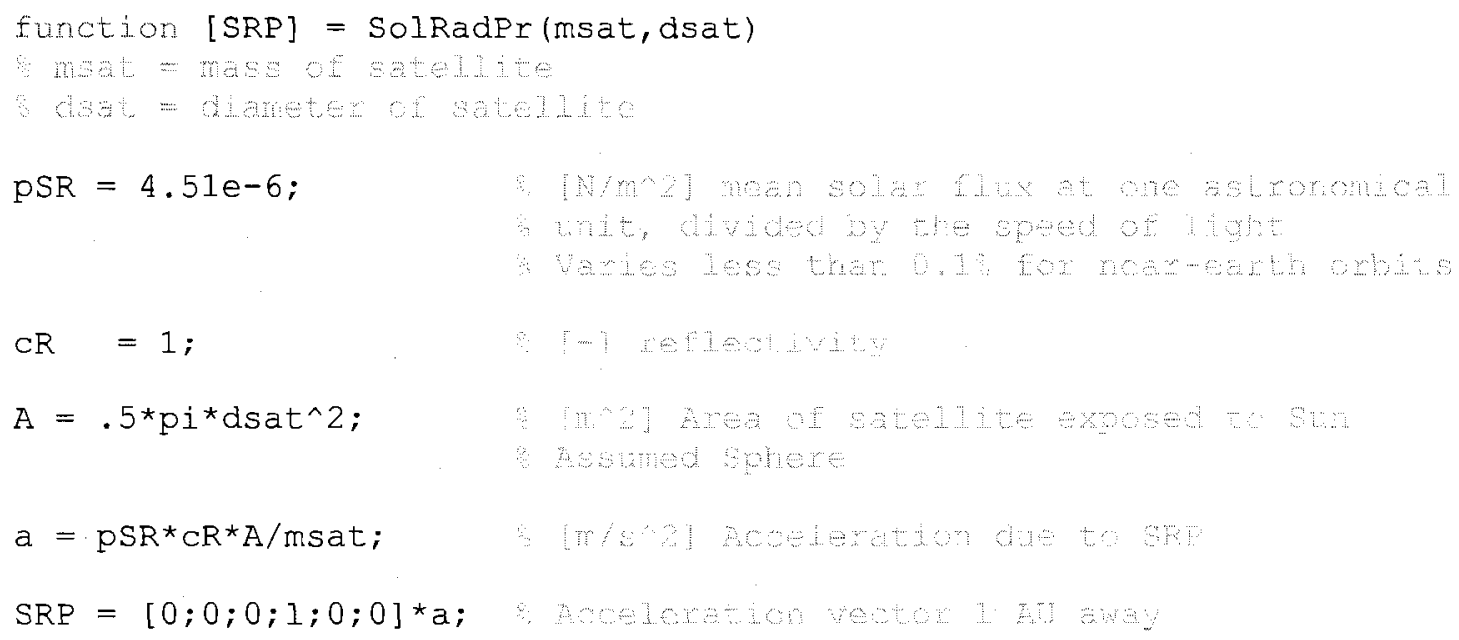




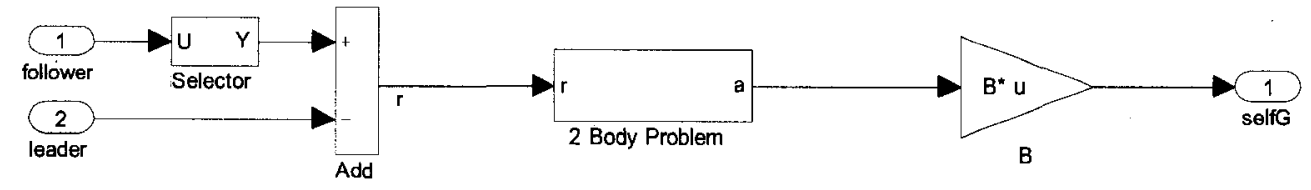

Figure A7 - Self-Gravity

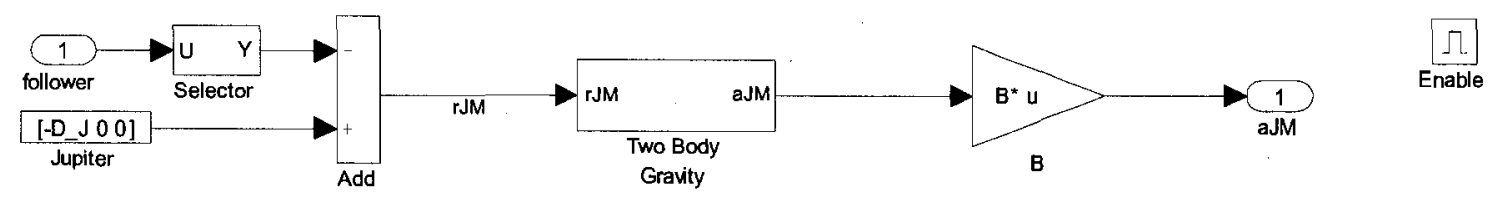

Figure A8 - Jupiter

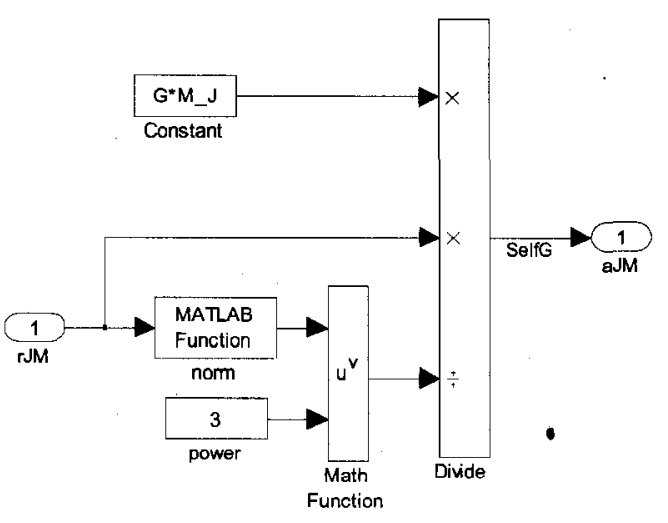

Figure A9 - Two-body gravity problem

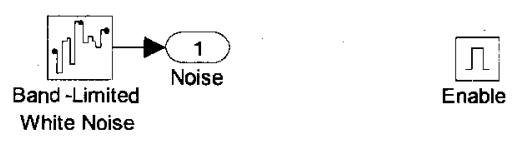

Figure A10 - Measurement noise 


\section{A.4 Common m-File Code}

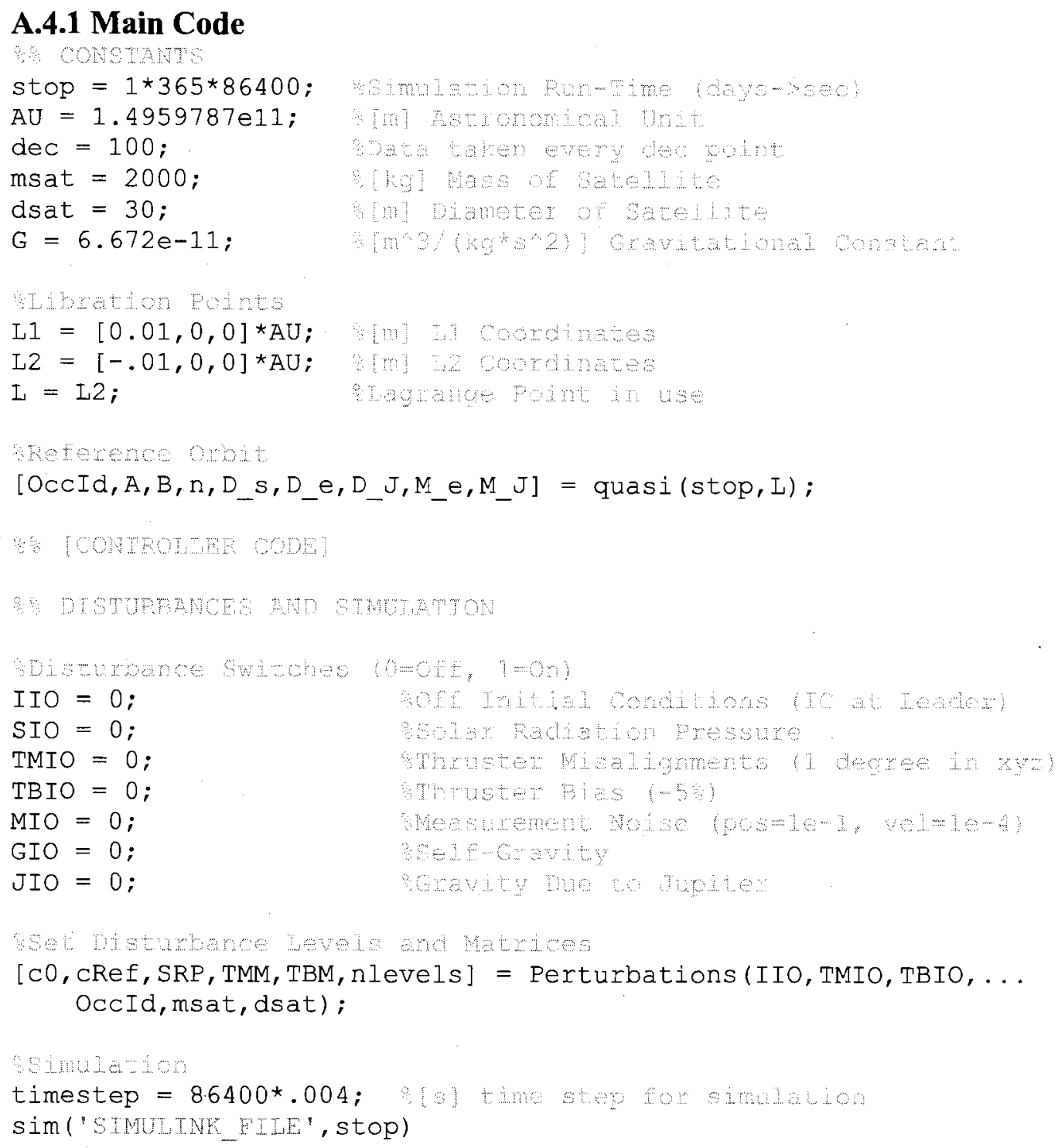

\section{A.4.2 Reference Orbit Code}

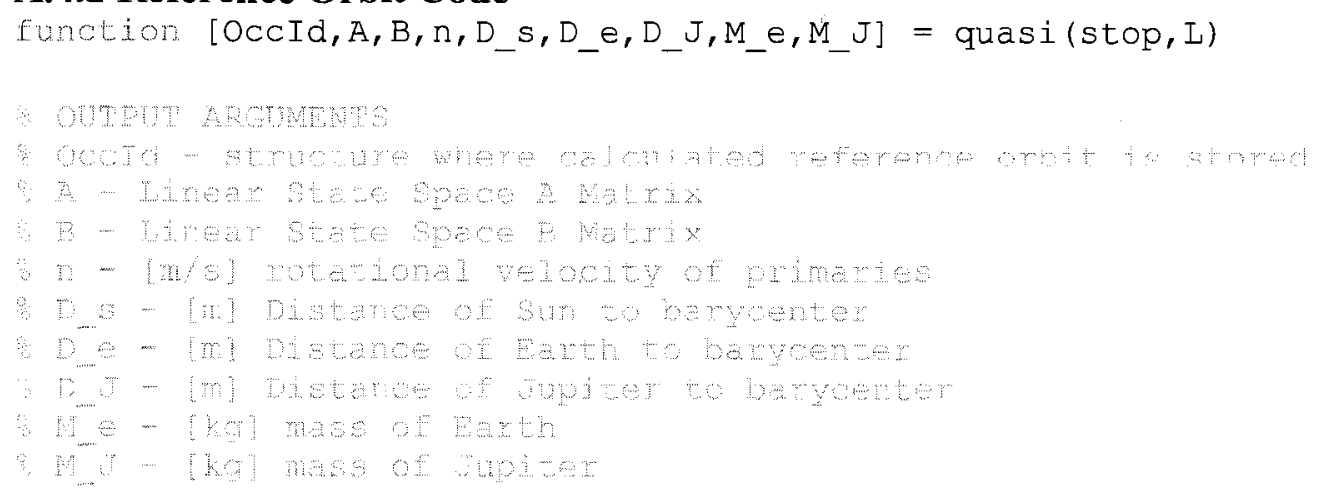




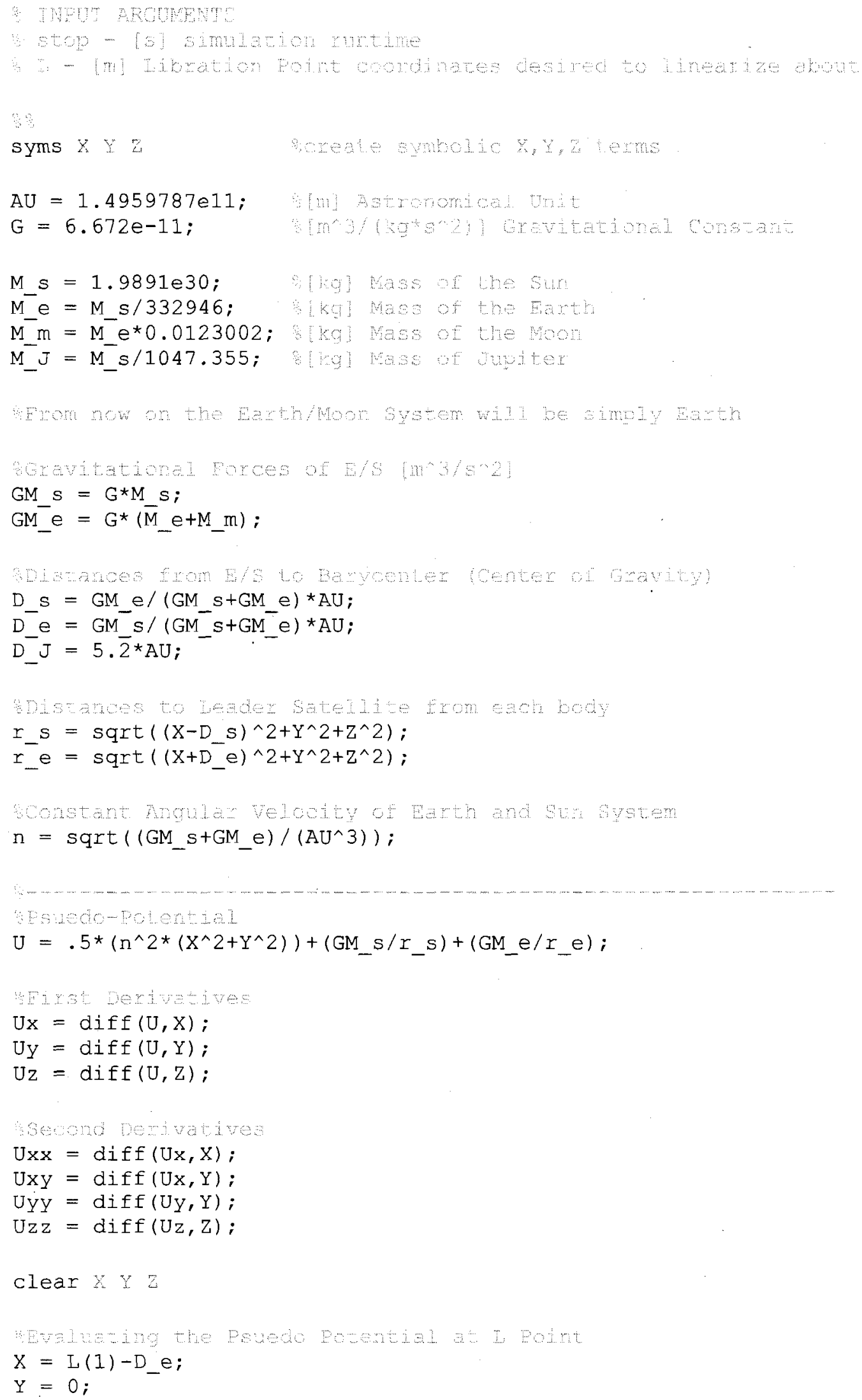




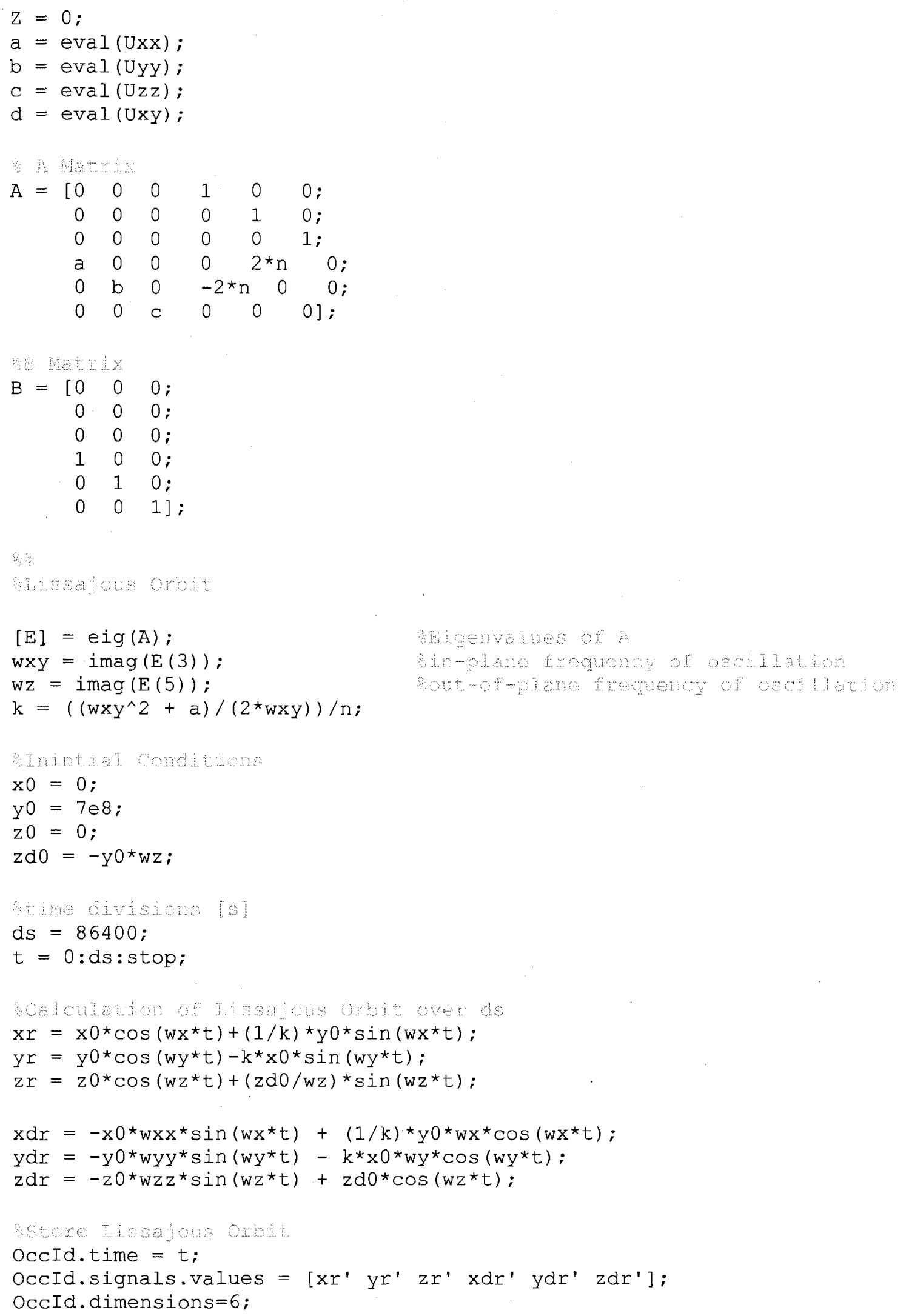




\section{APPENDIX B}

\section{PID MODELS}

\section{B.1 SIMULINK Model}

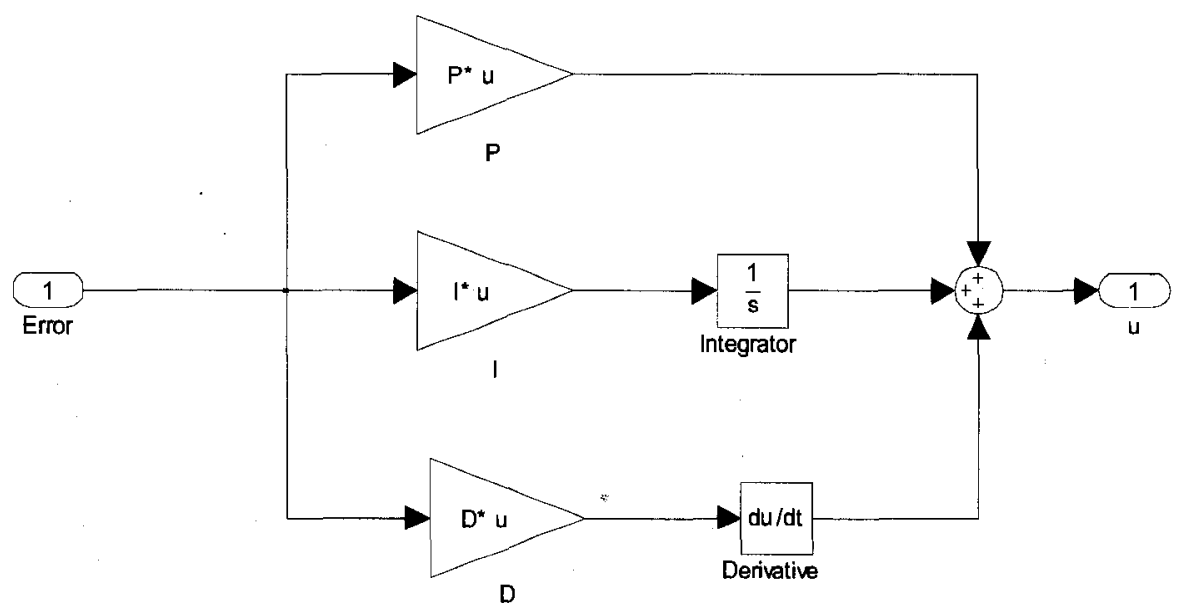

Figure B1 - PID controller model

\section{B.2 m-File Code}

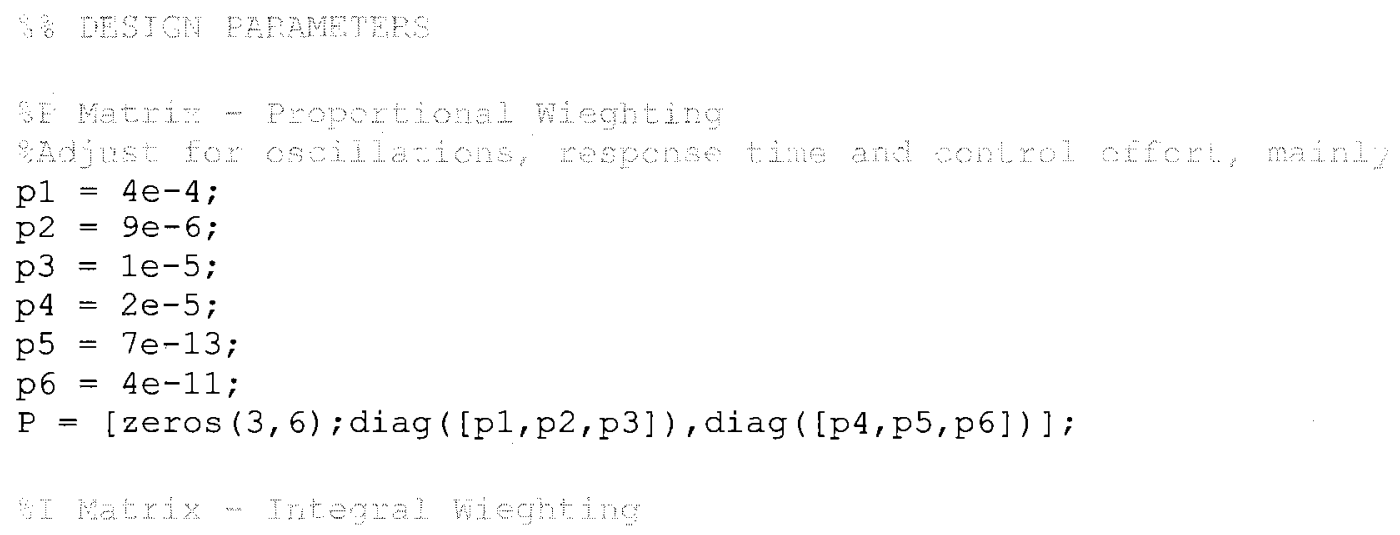




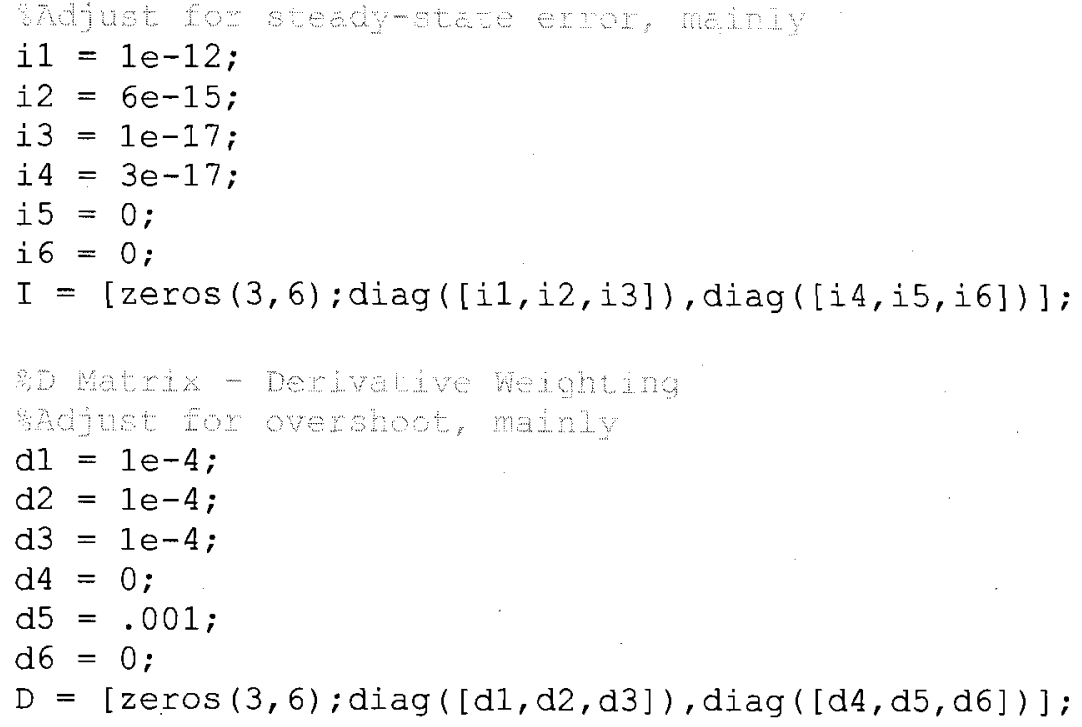




\section{APPENDIX C}

\section{LQR MODELS}

\section{C.1 SIMULINK Model}

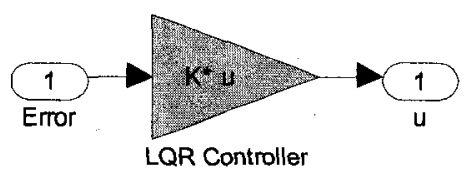

Figure C1 - LQR controller model

\section{C.2 m-File Code}

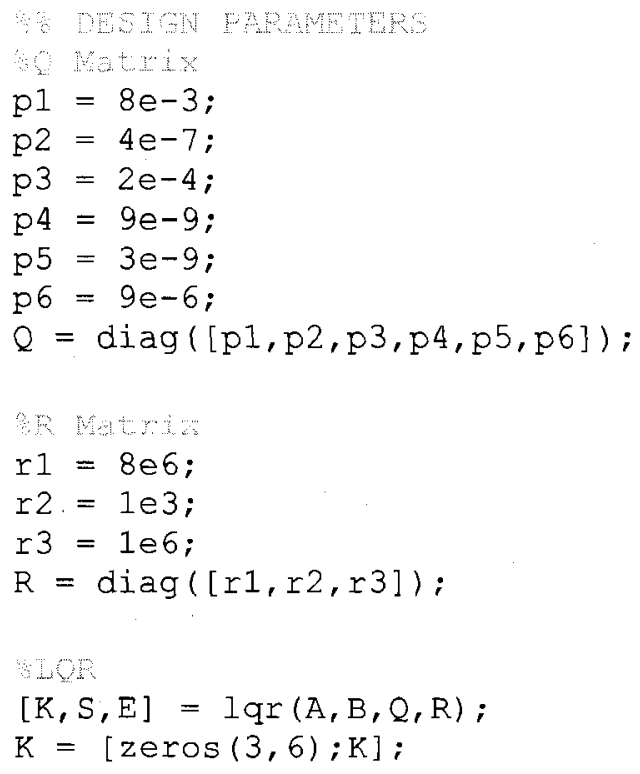




\section{APPENDIX D}

\section{H-INFINITY MODELS}

\section{D.1 SIMULINK Model}

$\mathrm{H} \infty$ design is modeled slightly differently than the other three controllers but still follows the same principles.

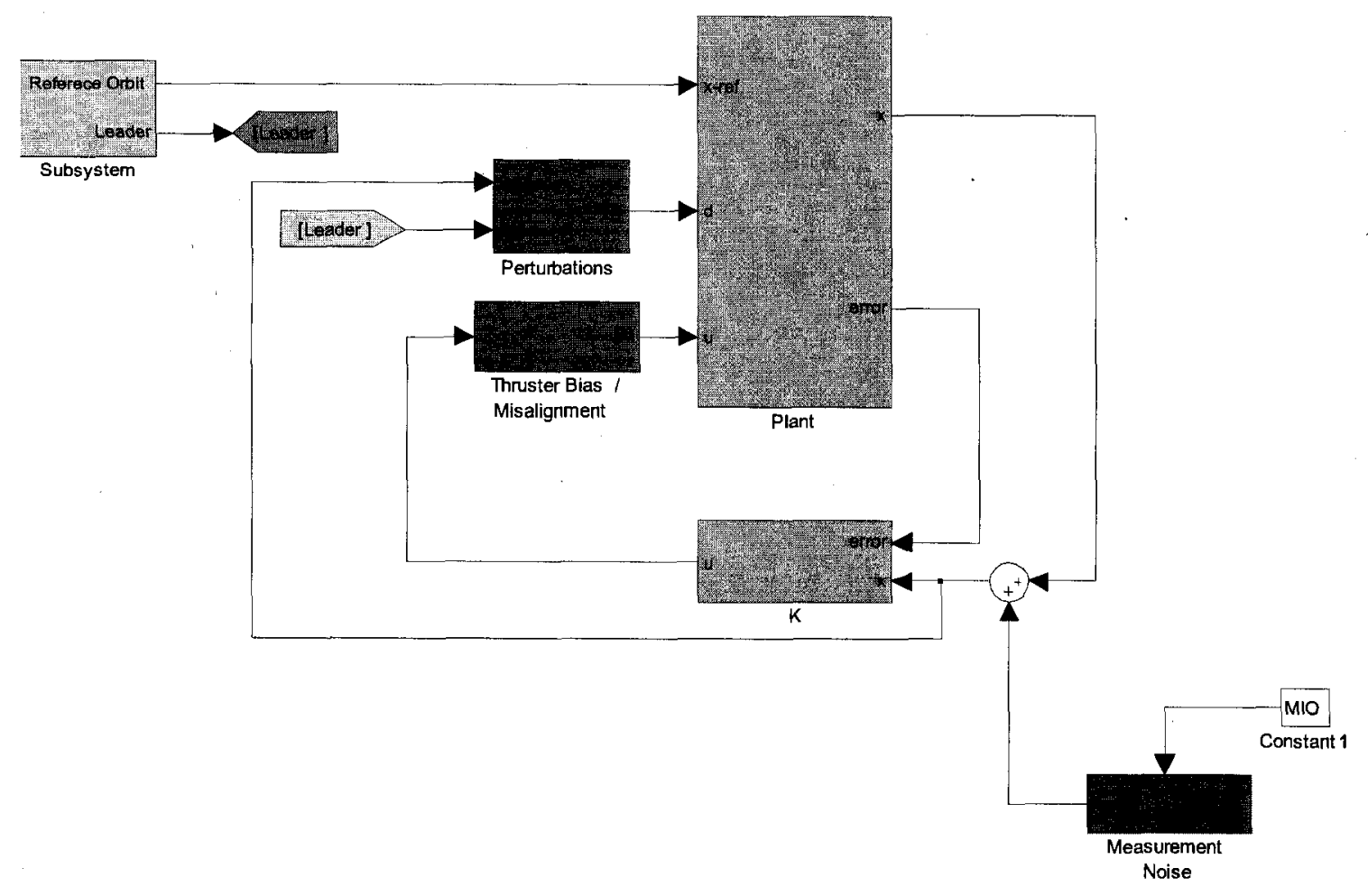

Figure D1 - $H_{\infty}$ simulink model 


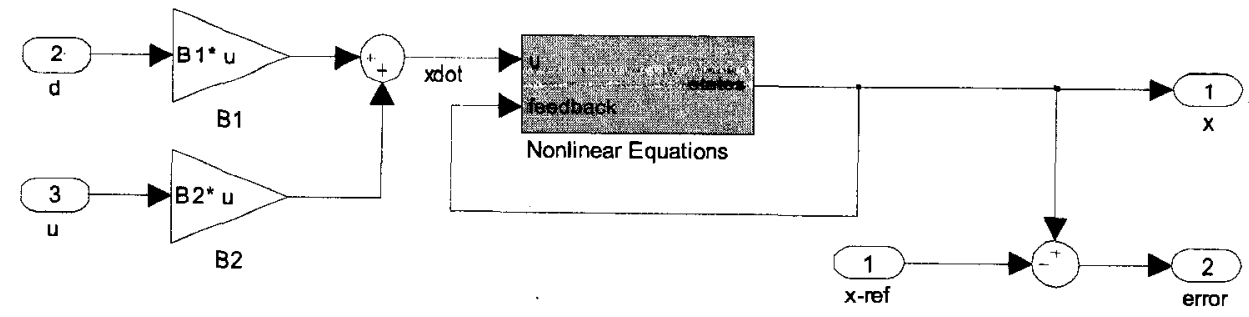

Figure D2 - $\mathbf{H}_{\infty}$ plant

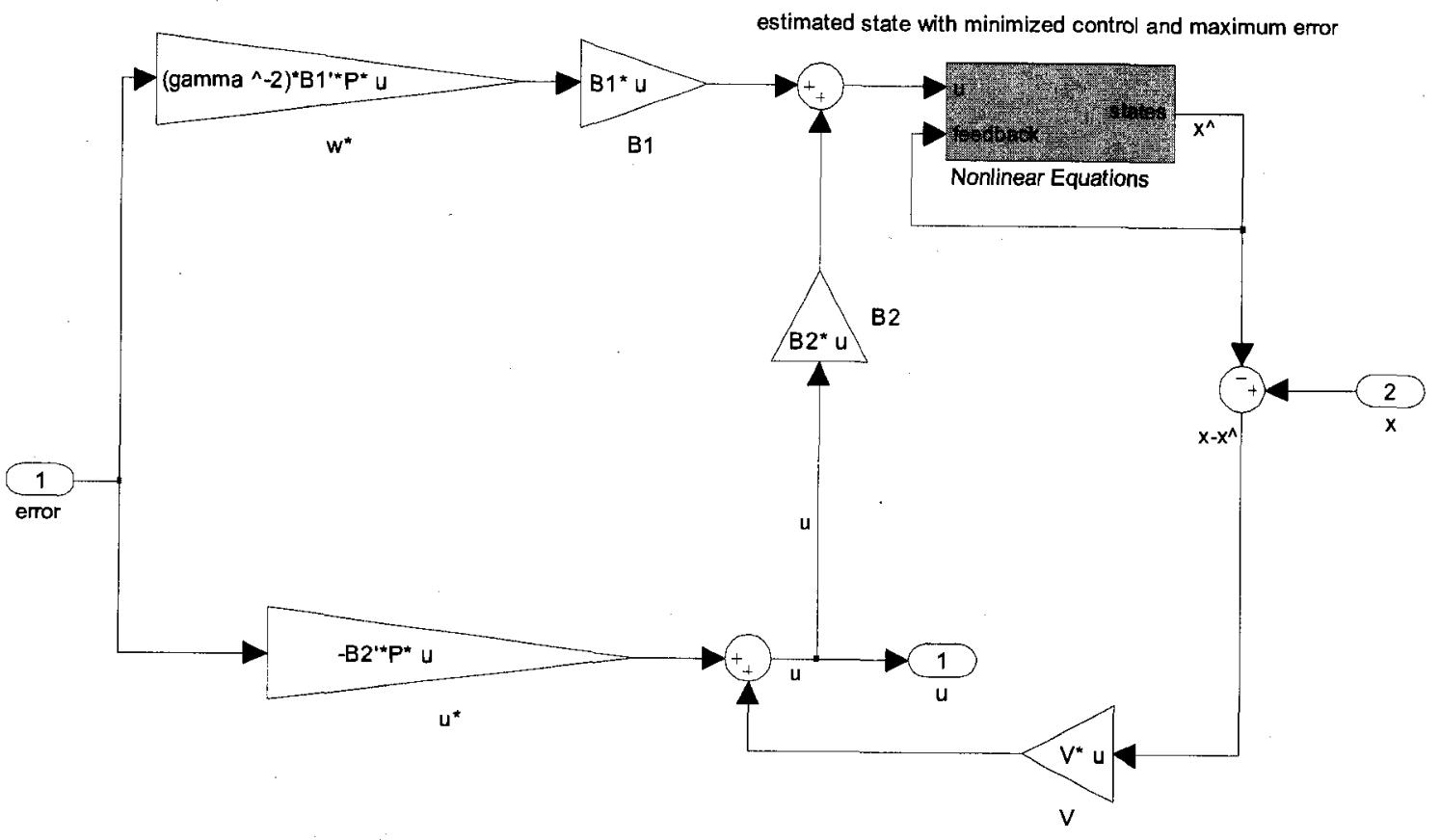

Figure D3 - $\mathbf{H}_{\infty}$ controller model

\section{D.2 m-File Code}

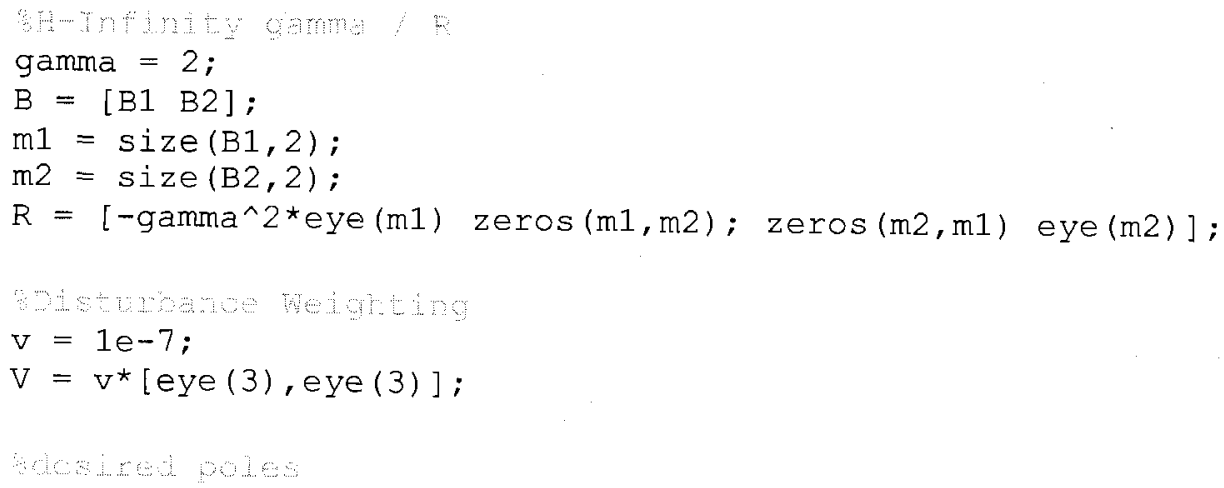




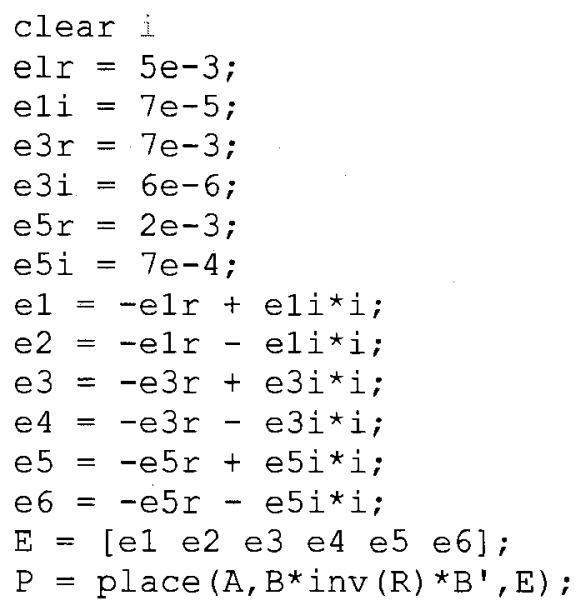




\section{APPENDIX E}

\section{SMC MODELS}

\section{E.1 SIMULINK Model}

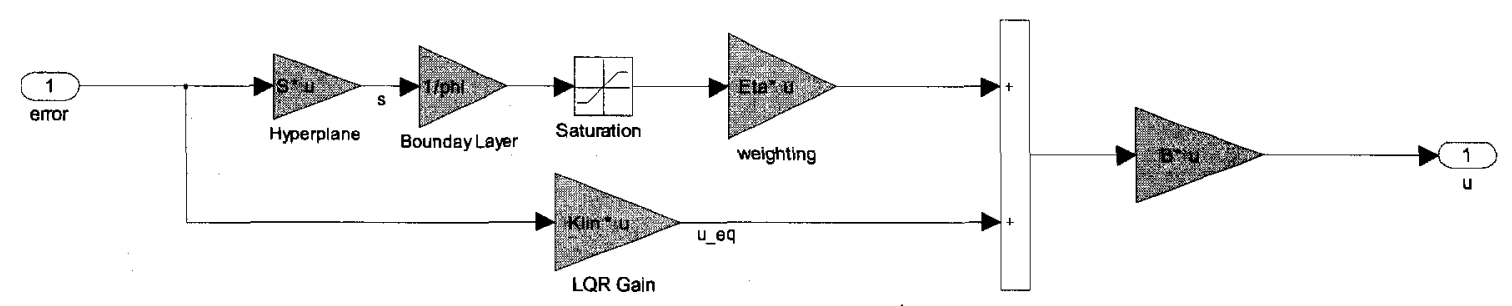

Figure E1 - SMC controller model

\section{$\underline{\text { E.2 m-File Code }}$}

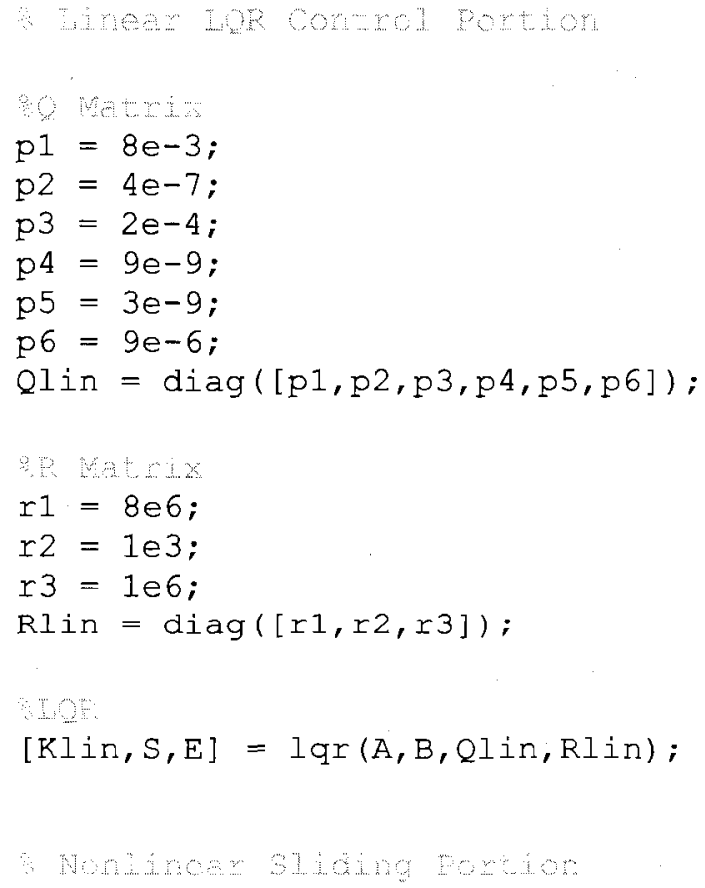




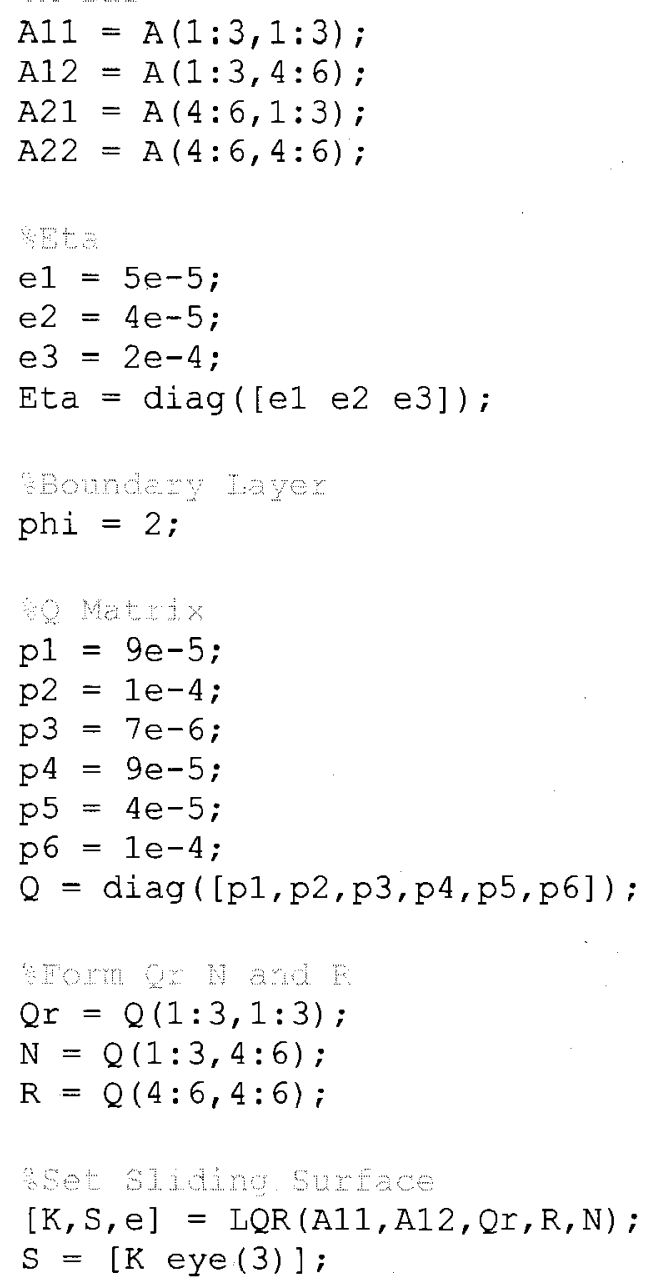




\section{APPENDIX F}

\section{LINEAR SYSTEM RESULTS}

The following tables present the results of the spacecraft system following the linearized dynamics of the CR3BP equations of motion. The results are for one year in orbit. These results are used as a preliminary step to obtain starting gains for controllers used with the nonlinear system. These tables should not be used to make conclusive observations about the control techniques.

\begin{tabular}{|c|c|c|c|c|c|c|c|}
\hline & \multicolumn{3}{|c|}{ Max Position Errors $[\mathrm{m}]$} & \multicolumn{3}{|c|}{ Max Velocity Errors [m/s] } & \multirow{2}{*}{ 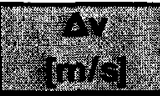 } \\
\hline & $\mathbf{x}$ & $y$ & 2 & $x$ & $y=$ & $z=z$ & \\
\hline Unperturbed & 0 & 0 & 0 & 0 & 0 & 0 & 0 \\
\hline Thr. Misalign. & 0 & 0 & 0 & 0 & 0 & 0 & 0 \\
\hline Thr. Bias & 0 & 0 & 0 & 0 & 0 & 0 & 0 \\
\hline Self-Gravity & 0 & 0 & 0 & 0 & 0 & 0 & 0 \\
\hline Jupiter Gravity & 0.004 & $6.20 \mathrm{E}-03$ & 7445 & $1.10 \mathrm{E}-10$ & $1.40 \mathrm{E}-10$ & 0.0026 & 0.0016 \\
\hline SRP & 3.2 & $4.80 \mathrm{E}-07$ & 0 & $1.30 \mathrm{E}-10$ & $4.30 \mathrm{E}-11$ & 0 & 0.025 \\
\hline Meas Nolse & 0.09 & 0.054 & 0.03 & $9.40 \mathrm{E}-04$ & $9.40 \mathrm{E}-04$ & $9.40 \mathrm{E}-04$ & 1.80E-04 \\
\hline Differing ICs & $4.60 \mathrm{E}-06$ & $5.90 \mathrm{E}-07$ & $7.00 E+08$ & $1.00 \mathrm{E}-10$ & $5.20 \mathrm{E}-11$ & 279 & 0.82 \\
\hline Worst Case & 3.6 & 0.056 & $7.00 \mathrm{E}+08$ & $9.30 \mathrm{E}-04$ & $9.40 \mathrm{E}-04$ & 281 & 0.88 \\
\hline
\end{tabular}

Table F1 - PID results (linear)

\begin{tabular}{|c|c|c|c|c|c|c|c|}
\hline & \multicolumn{3}{|c|}{ Max Position Ernors [m] } & \multicolumn{3}{|c|}{ Max Velocity Errors [m/s] } & \multirow{2}{*}{ ming } \\
\hline & $\mathbf{x}$ & $y$ & 2 & $x$ & $y$ & 2 & \\
\hline Unperturbed & 0 & 0 & 0 & 0 & 0 & 0 & 0 \\
\hline Thr. Misalign. & 0 & 0 & 0 & 0 & 0 & $\overline{0}$ & 0 \\
\hline Thr Bias & 0 & 0 & 0 & 0 & 0 & 0 & 0 \\
\hline Self-Gravity & 0 & 0 & 0 & 0 & 0 & 0 & 0 \\
\hline Jupiter Gravity & 0.11 & 6.1 & 7445 & $6.60 \mathrm{E}-11$ & $1.50 E-05$ & 0.0026 & 0.0016 \\
\hline SRP & 1.7 & 0.18 & 0 & $4.50 \mathrm{E}-11$ & $6.80 E-07$ & 0 & 0.025 \\
\hline Meas. Nolse & 0.84 & 12.8 & 0 & 0.0011 & $7.70 E-05$ & 0 & 0.0029 \\
\hline Bifforing $1 \mathrm{Cs}$ & 0.0044 & 4923 & $7.00 \mathrm{E}+08$ & $1.90 E-08$ & 0.013 & 279 & 0.068 \\
\hline Worst Case & 2.8 & $1.30 E+05$ & $7.00 E+08$ & 0.0011 & 1.1 & 281 & 0.096 \\
\hline
\end{tabular}

Table F2 - LQR results (linear) 


\begin{tabular}{|c|c|c|c|c|c|c|c|}
\hline & \multicolumn{3}{|c|}{ Max Position Errors [m] } & \multicolumn{3}{|c|}{ Max Velocity Errors $[\mathrm{m} / \mathrm{s}]$} & \multirow{2}{*}{ 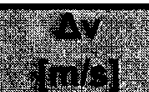 } \\
\hline & $x$ & y & 2 & $x$ & $y$ & 2 & \\
\hline Unperturbed & 0 & 0 & $\overline{0}$ & 0 & 0 & 0 & 0 \\
\hline Thr. Mtsalign: & $\overline{0}$ & $\overline{0}$ & $\overline{0}$ & $\overline{0}$ & $\overline{0}$ & $\overline{0}$ & $\overline{0}$ \\
\hline Thr Blas & 0 & 0 & 0 & 0 & 0 & 0 & 0 \\
\hline Self Gravity & 0 & 0 & 0 & 0 & 0 & 0 & 0 \\
\hline Jupiter Gravity & 0.0043 & 0.0062 & 0.11 & $1.90 \mathrm{E}-07$ & $2.60 \mathrm{E}-07$ & $2.30 \mathrm{E}-06$ & 0.0016 \\
\hline SRP & 0.064 & 0.095 & 1.6 & $2.90 \mathrm{E}-06$ & $3.90 \mathrm{E}-06$ & $3.50 \mathrm{E}-06$ & 0.025 \\
\hline Meas. Nolse & $1.30 \mathrm{E}-04$ & $2.50 \mathrm{E}-05$ & $1.50 \mathrm{E}-04$ & \begin{tabular}{|l|}
$7.60 \mathrm{E}-07$ \\
\end{tabular} & $6.60 \mathrm{E}-08$ & $6.90 \mathrm{E}-08$ & $3.30 \mathrm{E}-06$ \\
\hline Diftering 10 s & 4825 & 991 & 6753 & 0.24 & 0.05 & 0.34 & 0.26 \\
\hline Merst Caso & 5112 & 1055 & 7215 & 0.26 & 0.053 & 0.36 & 0.27 \\
\hline
\end{tabular}

Table F3 - $\mathrm{H}_{\infty}$ results (linear)

\begin{tabular}{|c|c|c|c|c|c|c|c|}
\hline & \multicolumn{3}{|c|}{ Max Position Errors [m] } & \multicolumn{3}{|c|}{ Max Velocity Errors [m/s] } & \multirow{2}{*}{ (3) } \\
\hline & $x$ & $y$ & 2 & $x$ & $y$ & 2 & \\
\hline Unperturbed & 0 & 0 & 0 & 0 & 0 & 0 & 0 \\
\hline Thr. Misalign. & 0 & 0 & $\overline{0}$ & 0 & 0 & 0 & $\overline{0}$ \\
\hline Thr. Blas & 0 & $\overline{0}$ & 0 & $\overline{0}$ & 0 & 0 & $\overline{0}$ \\
\hline Selforavity & 0 & 0 & 0 & 0 & 0 & 0 & 0 \\
\hline Supiter Gravity & 0.16 & 0.0018 & 0.0094 & $7.60 \mathrm{E}-05$ & $2.50 \mathrm{E}-07$ & $7.40 \mathrm{E}-09$ & 0.0016 \\
\hline SRP & 2.4 & $9.50 \mathrm{E}-04$ & 0 & $1.10 \mathrm{E}-03$ & $5.30 \mathrm{E}-07$ & $0.00 E+00$ & 0.025 \\
\hline Meas. Noise & $1.60 \mathrm{E}+00$ & $2.50 \mathrm{E}-01$ & $1.80 \mathrm{E}-01$ & $2.70 \mathrm{E}-03$ & $9.20 \mathrm{E}-04$ & 9.50E-05 & $5.70 \mathrm{E}-03$ \\
\hline Differing $1 \mathrm{Cs}$ & 567 & 3811 & 4987 & 0.69 & 0.037 & 0.063 & 0.13 \\
\hline Worst Case & 8.1 & 3637 & 6701 & 0.0061 & 0.042 & 0.067 & 0.068 \\
\hline
\end{tabular}

Table F4 - SMC results (linear) 